GEOLOGICAL SURVEY CIRCULAR 377

\title{
FLOODS OF AUGUST 1955 \\ IN THE NORTHEASTERN STATES
}



MERVIN S. PEI L.... EN

Flood Specialist

UNITED STATES DEPARTMENT OF THE INTERIOR

Douglas McKay, Secretary

GEOLOGICAL SURVEY

Thomas B. Nolan, Director

GEOLOGICAL SURVEY CIRCULAR 377

\title{
FLOODS OF AUGUST 1955 IN THE NORTHEASTERN STATES
}

\author{
Prepared by Water Resources Division
}

Washington, D. C., 1956

Free on application to the Geological Survey, Washington 25, D. C. 


\section{PREFACE}

This preliminary report on the floods of August 1955, has been prepared by the U. S. Geological Survey, Water Resources Division, C. G. Paulsen, chief, under the general direction of J. V. B. Wells, chief, Surface Water Branch. Much of the collection of the basic streamflow records by the Geological survey has been made in financial cooperation with public agencies in the States in the area of major flooding, under the direction of the following district engineers: Massachusetts, Rhode Island, H. B. Kinnison; Connecticut, B. L. Bigwood; New York, A. W. Harrington; New Jersey, O. W. Hartwell; Pennsylvania, J. W. Mangan.

Because of the urgent need to perform the field work and the office computations as promptly as possible,many hydraulic engineers from all sections of the country were brought to the flood area. The assignment of the additional personnel, much of the work of processing computations of peak discharges by indirect methods, and the assembling of this report were performed by hydraulic engineers of the branch staff, under the supervision of Tate Dalrymple, chief, Technical Standards Section.

Several Federal and State agencies contributed technical data. The isohyetal maps were adapted from maps furnished by the U. S. Weather Bureau. Damage figures are those provided by the Business and Defense Services Administration.

II 


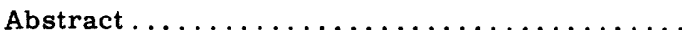

Introduction . . . . . . . . . . . . . . .

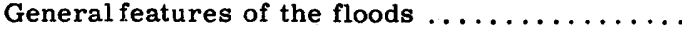

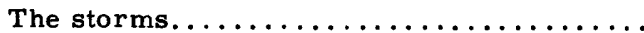

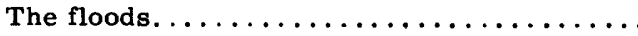

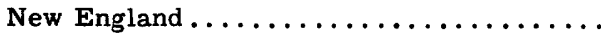

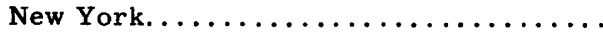

New Jersey ......................

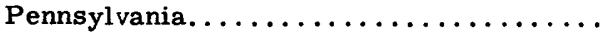

Summary of flood damage ..............

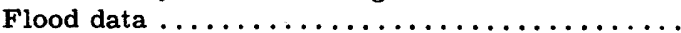

Measurement of flood discharges .........

Summary of stages and discharges........

Gaging station records...............

Merrimack River basin:

Concord River below River Meadow Brook, at Lowell, Mass. .............

Charles River basin:

Charles River at Charles River Village,

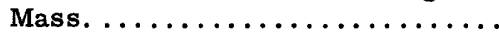

Providence River basin:

Blackstone River at Woonsocket, R. I. ...

Thames River basin:

Willimantic River near South Coventry, Conn. .................. 38

Quinebaug River at Westville, Mass..... 38

French River at Webster, Mass. ........ 39

Quinebaug River at Jewett City, Conn.... 40

Connecticut River basin:

Connecticut River at Montague City, Mass. 41

Ware River at Gibbs Crossing, Mass. .... 42

Quaboag River at West Brimfield, Mass... 42

Chicopee River at Indian Orchard, Mass. . 43

Middle Branch Westfield River at Goss

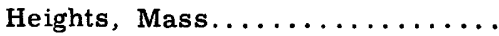

West Branch Westfield River at Huntington,

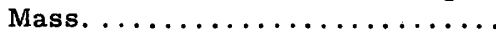

Westfield River near Westfield, Mass. ...

Connecticut River at Thompsonville, Conn.

Scantic River at Broad Brook, Conn.....

West Branch Farmington River near New Boston, Mass. ............ 48

Still River at Robertsville, Conn. ...... 49
Flood data--Continued

Gaging station records--Continued

Connecticut River basin--Continued

Burlington Brook near Burlington, Conn. .. 50

Farmington River at Rainbow, Conn. .... 50

Housatonic River basin:

Housatonic River at Gaylordsville, Conn... 51

Pomperaug River at Southbury, Conn. .... 51

Naugatuck River near Thomaston, Conn. . . 52

Hudson River basin:

Rondout Creek near Lackawack, N. Y. ... 52

Rondout Creek at Rosendale, N. Y. .... 53

Wallkill River at Gardiner, N. Y. ...... 54

Wappinger Creek near Wappingers Falls,

N. Y. .............. 55

Raritan River basin:

South Branch Raritan River at Stanton, N. J. 56

Delaware River basin:

Delaware River above Lackawaxen River, near Barryville, N. Y. ...... 57

Middle Creek near Hawley, $\mathrm{Pa} . \ldots \ldots \ldots .58$

Lackawaxen River at Hawley, Pa. ...... 59

Delaware River at Port Jervis, N. Y. .... 60

Neversink River at Oakland Valley, N. Y. 61

Delaware River at Montague, N. J. . . . . . 62

Bush Kill at Shoemakers, $\mathrm{Pa} . . . \ldots \ldots \ldots .62$

Flat Brook near Flatbrookville, N. J. .... 63

Brodhead Creek at Minisink Hills, Pa.... 64

Paulins Kill at Blairstown, N. J. ....... 65

Delaware River at Belvidere, N. J. ..... 66

Lehigh River at Stoddartsville, Pa. ..... 66

Lehigh River at Tannery, $\mathrm{Pa} . \ldots \ldots \ldots \ldots 67$

Lehigh River at Walnutport, $\mathrm{Pa} . \ldots \ldots \ldots 68$

Lehigh River at Bethlehem, Pa........ 69

Musconetcong River near Bloomsbury, N. J. 70

Delaware River at Riegelsville, N. J. ... 71

Tohickon Creek near Pipersville, $\mathrm{Pa} . \ldots .72$

Delaware River at Trenton, N. J. ...... 73

Neshaminy Creek near Langhorne, Pa..... 74

Little Schuylkill River at Tamaqua, $\mathrm{Pa} . . .74$

Susquehanna River basin:

Lackawanna River at Old Forge, Pa. ... 75

Wapwallopen Creek near Wapwallopen, Pa.. 76

\section{ILLUSTRATIONS}

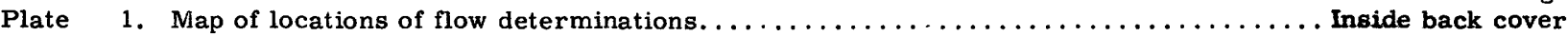

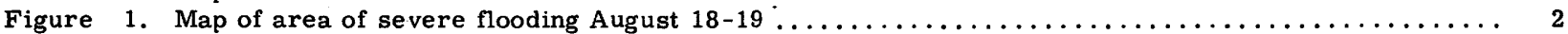

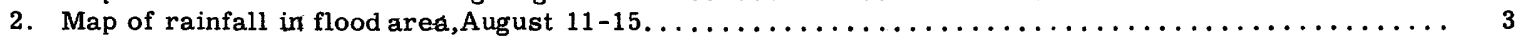

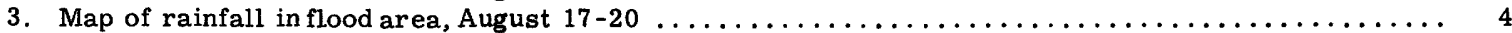

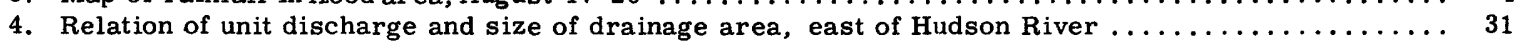

5. Relation of unit discharge and size of drainage area, west of Hudson River ............. 32

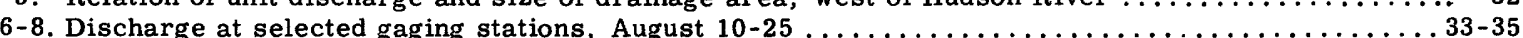

TABLES

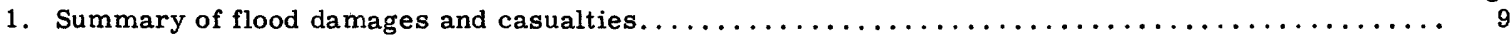

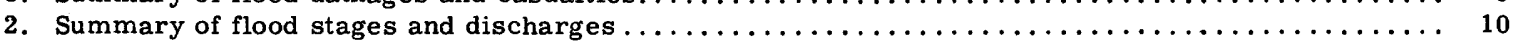





\title{
FLOODS OF AUGUST 1955 IN THE NORTHEASTERN STATES
}

\author{
Prepared by Water Resources Division
}

\section{ABSTRACT}

The floods of August 1955 were an unprecedented disaster in a large area of the northeastern United States. They rank among the most destructive in the country's history. Augmented by the antecedent hurricane storm of August 11-15, the rainfall of August 17-20 accompanying hurricane Diane reached maximum values of 17 to 19 inches in south-central Massachusetts. Record-breaking floods resulted within a broad region extending from southeastern Pennsylvania to eastern Massachusetts.

The floods were outstanding in four categories: The large geographic area covered by floods of such magnitude; the extensive damage and loss of life ranking with the greatest recorded in this country; the degree to which prior records were exceeded; and the distribution which was such that the greatest floods occurred predominately on the smaller streams. Property damage has been estimated to be about half a billion dollars and was mostly concentrated in the heavily industrialized valleys of New England. A death toll of 179 persons was attributed to the floods. Peak discharges exceeded previously established maxima by 2.2 and 2.3 times respectively, on Blackstone River at Woonsocket, R. I. , and Quinebaug River at Putnam, Conn.; 4.1 times on Naugatuck River near Thomaston, Conn.; and 4.5 times on Bush Kill at Shoemakers, Pa. A unit runoff of 2,300 cubic feet per second per square mile occurred from 2.50 square miles on Powdermill Brook near Westfield, Mass. Although the floods were generally greatest on the smaller streams, Connecticut River at Hartford reached the third highest stage since settlement and Delaware River between Port Jervis and Trenton exceeded the previous historic flood of 1903.

This advance report has been prepared to supply preliminary information needed for immediate planning. It has been released pending preparation of a more comprehensive report covering a three-month period of floods within an area from Massachusetts to North Carolina. Included herein, for the region from Massachusetts to Pennsylvania, are general descriptions of the floods, peak discharges for the present and previous record floods at gaging stations, peak discharges at many miscellaneous sites in the areas of greatest flooding, and detailed stage and discharge data at 51 selected gaging stations.

\section{INTRODUCTION}

The floods of mid-August 1955 in the northeastern part of the United States rank among the most destructive floods experienced in the country. Caused by storms of tropical origin, the floods were of disaster proportions in a broad arc from south of Philadelphia to Boston. The loss of life and the property damage were great because of the relatively thick settlement of the valleys and the concentration of industries along the streams.

Actually, there were two sets of floods in August 1955. The first, resulting from hurricane Connie, occurred mostly in the coastal plain, from North Carolina to New England, on August 12-13. The second set of floods were those of August 18-19, following hurricane Diane. They affected a zone farther inland but essentially concentric with the coastline (see fig. 1).

This report covers the floods of August 18-19, principally, because of the great destruction wrought by those floods and because the need exists for some data on them at an early date. Reference is made in the report to a limited amount of data for the earlier floods in August as collateral information--the first floods set the stage for the second floods and in some places the first floods were the greater.

The technical evaluation of the floods of August 18-19 and the reporting of them were complicated by the occurrence of other major floods in the northeastern States on October 15-16, and by lesser floods on October 5 , on October 30 , and on November 5 . In addition, the eastern part of North Carolina had severe floods September 20. The complex interrelations of the four major floods, the overlapping areas involved, and the timing of the floods, made a comprehensive preliminary report impracticable. A report is in preparation (December 1955) that will cover the three-month flood period and the area from North Carolina to Mas sachusetts.

The purpose of this advance report is to present a sampling of the streamflow records in the area of severe flooding in the August 18-19 floods. Peak stage and discharge data are presented for most of the gaging stations and for many miscellaneous sites in the report area. Maxima are shown also for the highest previous floods of record, to provide a basis for evaluating the present floods. Detailed data are listed for selected gaging stations, that will enable the user to construct accurate discharge hydrographs. Several assemblies of information are shown in graphical form but detailed analyses of the flood data have not been made. The data for the present flood are preliminary and are subject to possible revision following further review.

storms of trgpical origin, the floods were of disaster 


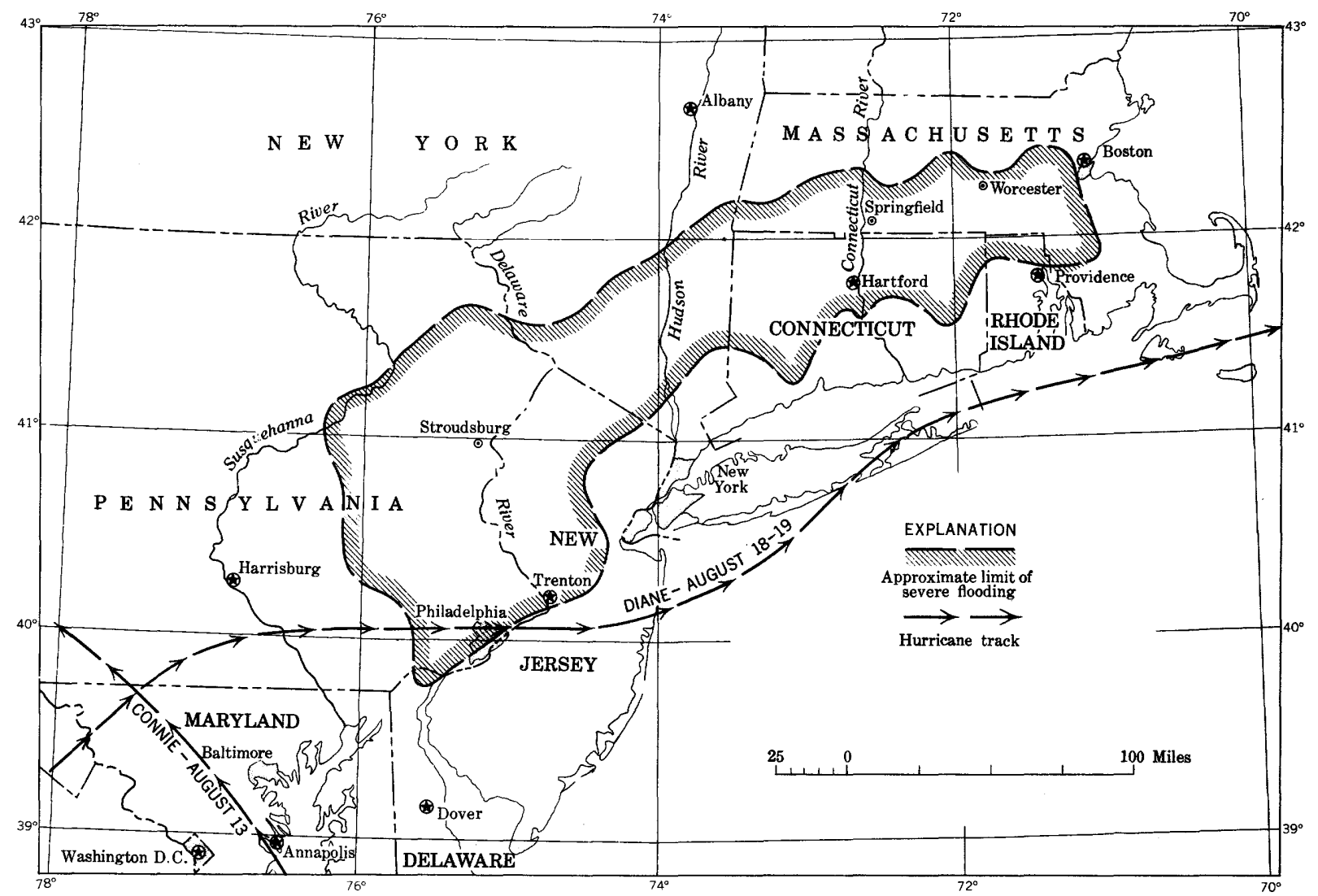

Figure 1.--Map of the area of severe flooding August 18-19, showing tracks of the August hurricanes, Connie and Diane.

\section{GENERAL FEATURES OF THE FLOODS}

\section{The Storms}

As the summer of 1955 progressed, it was marked by above-average temperatures and by the lack of rainfall. Drought conditions developed in large areas of New England and the Middle Atlantic States, and by the end of July the outlook for adequate water supply and abundant crops was poor. Light to moderate rains oc curred in the period August 5-8 over most of the northeastern States, but drought conditions continued.

The drought was dramatically broken by the heavy rains that accompanied the passage of hurricane Connie August 12 and 13. The storm entered the mainland at the North Carolina capes and followed a general northwesterly course across central and western Pennsylvania to the westerly tip of New York (fig. 1). In the period August 11-15, rainfall was heavy over a large area and some totals were outstanding: in western Massachusetts and in western Connecticut, more than 8 inches; in the Catskill Mountains region, more than 15 inches at one station, nearly 13 inches on Long Island; as much as 11 inches in northern New Jersey; and more than 12 inches in southeastern Pennsylvania. The distribution of the rainfall is shown in figure 2.

On August 18 and 19, hurricane Diane moved northward across Virginia with diminıshing wind velocities, turned sharply to the east across the southern edge of Pennsylvania and across New Jersey south of Trenton as shown in figure 1, then went to sea, just skirting Long Island and the southern New England coast.

The rainfall associsted with the Diane storm was heaviest alono an inland arc from Philadelphia to the Boston area. Amounts of rain were large in eastern Pennsylvania, notably in the Pocono Mountains region, and in the southern part of the Catskill Mountains region. The greatest rainfall occurred in a band along the southern third of Massachusetts and in northwestern Connecticut: over 16 inches south of Boston; more than 17 inches near Worcester; and nearly 20 inches in the Westfield River basin, west of Springfield. The distribution of the rainfall in the period August 17-20 is shown in figure 3 .

For more detailed information on the Diane storm and on precipitation in the northeastern States, the investigator is referred to publications of the U. S. Weather Bureau $1 /$.

\section{The Floods}

Damaging floods occurred in many streams as a result of the Connie storm and these will be described in the comprehensive flood report now in preparation. That the floods were not worse may be ascribed to the antecedent dry condition of the soil and the relatively

1/U. S. Weather Bureau, 1955, Preliminary report of hurricane Diane and floods in Northeast--August 1955; Hurricane rains and floods of August 1955, preliminary precipitation data. 


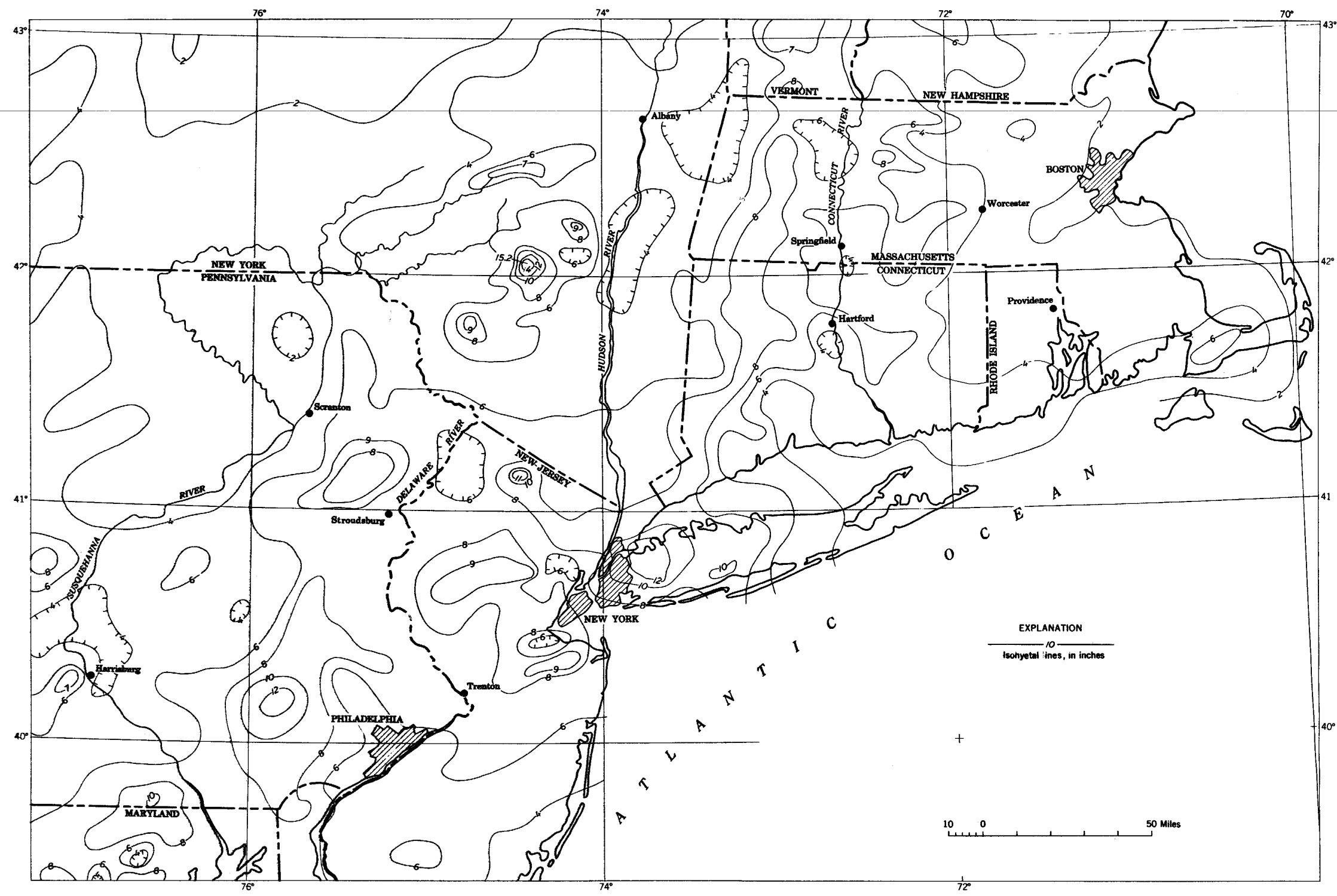

Figure 2. --Map of the flood area, showing total rainfall in the period August 11-15, 1955. (Adapted from map furnished by U. S. Weather Bureau). 


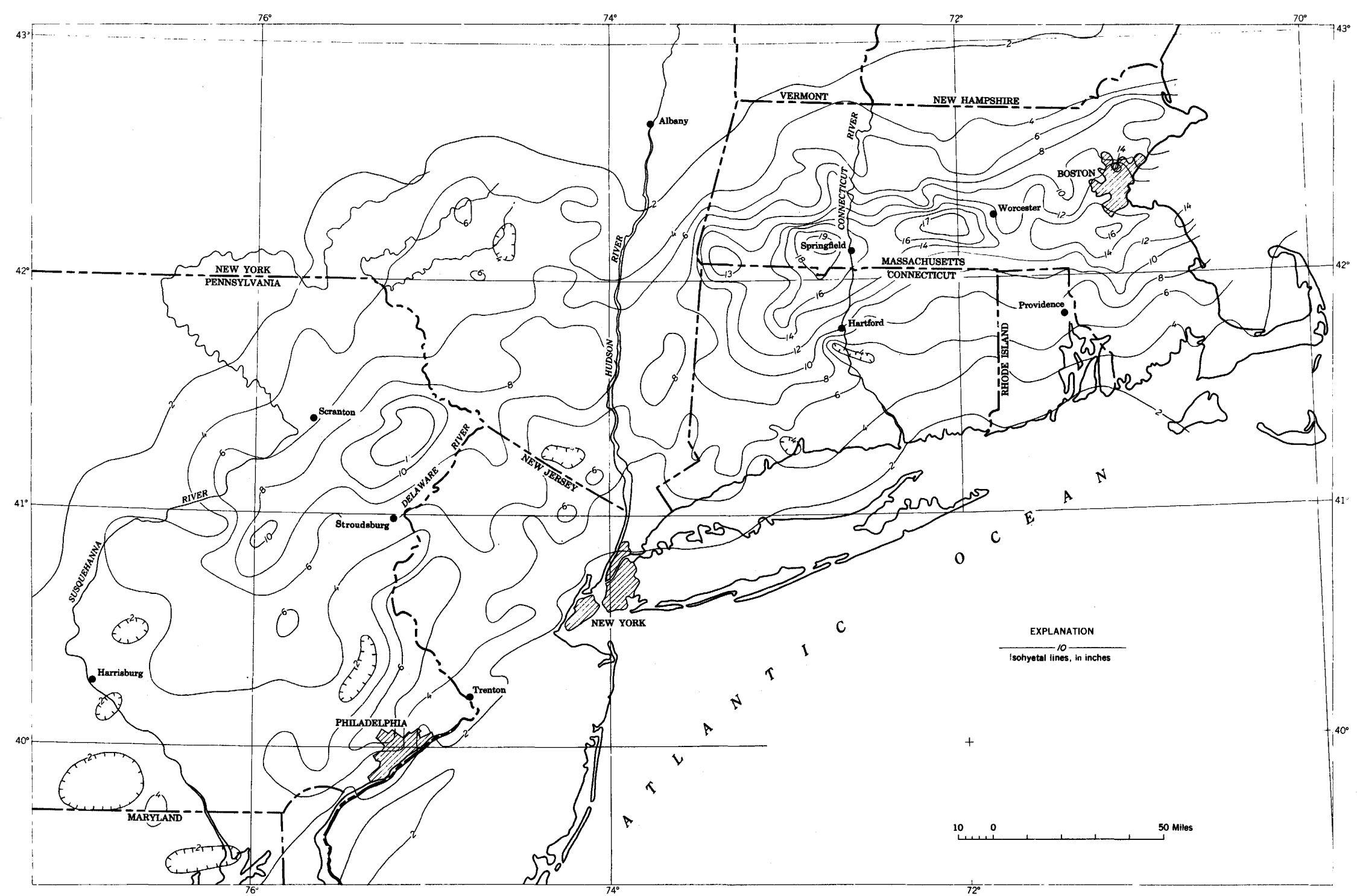

Figure 3. --Map of the flood area, showing total rainfall in the period August 17-20, 1955. (Adapted from map furnished by U. S. Weather Bureau). 
large amount of infiltration. The soil became saturated, however, and the base flow in the streams was notably higher after Connie had passed.

What can be described only as a special kind of calamity occurred in the northeastern States, August 18 and 19,1955 . As the torrential rains fell on an almost saturated soil in an area of rapid runoff, the streams rose with terrifying rapidity, overflowed their banks, and flooded the steep valleys of Massachusetts, Connecticut, New York, New Jersey, and Pennsylvania.

The flood waters ravaged everything in their paths. In the forests the hillsides were eroded and many landslides occurred and in the fields the soil and crops were replaced by sand, gravel, and rocks as the waters cut new stream channels and reshaped old channels. Roads were completely destroyed for miles and hundreds of miles of roads required major repairs. Bridge piers and abutments were undermined and debris caught on them--debris including trailer trucks, railroad cars, and houses. Hundreds of bridges were destroyed and communication by highways became a major problem.

The disruption of rail communication likewise was a major problem. The railroads suffered great losses of track, bridges, rolling stock, and other items. Several branch lines had not been repaired by December 1955 and possibly will not be put back into service.

The early settlers built their homes in the valleys near the streams and the communities developed deep in the valleys. The floods smashed these homes and factories and villages. The dams that provided the hydraulic head for power became breached and added the contents of their reservoirs to the natural runoff. Where the flood waters coursed, nothing was spared-houses, stores, banks, supply yards, factories, schools, churches, and even cemeteries were destroyed. The debris resulting from this destruction became the ally of the water and helped to destroy things farther downstream.

Water mains, power lines, telephone and telegraph lines were damaged by the floods, and only radio remained as a means of communication in many places.

Normal activities practically ceased along the valleys. The first need was to evacuate people; nevertheless many perished. Public agencies and private groups acted swiftly and the story of the heroism and the rescues is an epic that is not a part of a report like this; nor is the story of the tremendous cleanup and rehabilitation job that was done.

From the hydrologic viewpoint, the floods of $\mathrm{Au}$ gust 18-19 were outstanding. New maximum stages and discharges were recorded and determined at many gaging stations, some of the stations having relatively long records. Based on the statements of old residents and on the historical records, the floods in some areas were the greatest known and rank among those that are identified as rare. It is evident, however, that consideration will have to be given to the possible. occur rence again of floods of this magnitude. The hydrologic data obtained during and after the floods can provide the basis of design for future development in much of the area that was flooded.
The chief characteristic of the August 18-19 floods was that they were tributary floods. None of the larger rivers in the area was wholly involved. Reference to figure 1 will show that the flood zone was in an irregular arc essentially normal to the large streams: the Connecticut, the Hudson, the Delaware, the Lehigh, and the Schuylkill. Where a sizable stream was almost wholly in the area of heavy rainfall, like Westfield River, Naugatuck River, and Brodhead Creek, the peak discharges and the amounts of damage were appalling. One can only speculate about what could have happened had the heavy rainfall occurred along the main stem of one of the larger rivers.

The rainfall pattern shows orographic influences where the moisture-laden air swirling in from the sea was forced to rise over the several mountain masses in the area. The relatively complex topography of the northeastern States also caused a high variability in the rainfall and the runoff within comparatively short distances. There are examples of extremely high flows in one basin and practically no storm reaction in the adjoining basin. This variability makes simple hydrologic comparisons somewhat difficult. Studies have not been made as yet that might show the regional characteristics of the floods, but it is suggested that the user consider the regional aspect rather than just the information collected at any one place. What happened in one small area could have been a topographic and geologic characteristic, but also might have been a freak of the storm.

In New England, maxima at many gaging stations exceeded those established in the flood of September 1938, which has been used extensively as the basis for the design of hydraulic structures. At stations along the main Delaware River all previous records were broken, and at places in nortizeastern Pennsylvania the flood of May 1942 was surpassed.

\section{New England}

At the northeasterly end of the flood area in Mas sachusetts, new highs occurred on such streams in the relatively flat coastal areas as Concord River, Charles River, Neponset River, Taunton River, and others. Damage was mostly from inundation, as compared with damage from fast-moving water.

Blackstone River heads in one of the areas where extremely heavy rainfall occurred during the Diane storm (see fig. 3), and the valleys in the basin are highly industrialized. Failures of dams augmented the natural peaks and the damage effect increased downstream. Velocity damage was great. Horseshoe Dam went out at Woonsocket and that city suffered a major disaster. The peak discharge of Blackstone River at Woonsocket was 2.2 times the peak during the 1938 flood and the stage was 7.4 feet higher.

All the streams that head in Massachusetts and flow generally southward into Connecticut, along the entire northern boundary of Connecticut, had severe floods. The upper tributaries and the entire main stem of Quinebaug River were subjected to new maximum flows. Nearly all the dams on French River were destroyed or badly damaged; the failure of one dam breached 
U. S. Highway 20, at West Auburn. Another large washout on this principal east-west route occurred at Charlton City, Mass., where a sudden surge occurred in Cady Brook as a result of the destruction of Glenecho Dam, about 2 miles upstream. The flood wave carried through to Southbridge, about 7 miles downstream, where Cady Brook joins Quinebaug River. At Southbridge the discharge of Cady Brook was about 26,300 cubic feet per second (cfs), from a drainage area of 12.0 square miles.

Putnam, Conn., was badly damaged by Quinebaug River. The gaging station there showed a peak discharge of $48,000 \mathrm{cfs}$, which was 2.3 times that in the 1938 flood, and the stage was about 7.0 feet higher than in 1938. Events of similar magnitude occurred throughout the Quinebaug basin, except for the tributaries from the east, which had relatively minor floods. Small tributaries of Willimantic River also were very high.

In the Chicopee River basin (which is tributary to Connecticut River), Chicopee River, Ware River, and Swift River did not reach flows as great as those in September 1938. Quaboag River and its tributaries, however, had extremely high peaks. For instance, Quaboag River at West Brimfield had a peak discharge of $12,800 \mathrm{cfs}-$-about 1.5 times the 1938 peak. The discharge from small tributaries was very large, with unit discharge of about $800 \mathrm{cfs}$ per square mile from drainage areas of less than 8 square miles. Blodgett Mill Brook caused a large amount of damage to the Massachusetts Turnpike, which was under construction. U. S. Highway 20 was inundated at several places, and dams, factories, houses, and all lines of communication received large amounts of damage.

The same pattern was repeated to the south in Connecticut, where Scantic River at Broadbrook, Conn., had a peak flow nearly twice that recorded in 1938 . Farther to the south, along the east side of Connecticut River, the flood effect was much less.

The main stem of Connecticut River itself had what might be called a midbasin flood; that is, the drainage area above the center of Massachusetts contributed relatively little flood water. Most of the flow in the middle reaches of the Connecticut came out of Chicopee River, Westfield River, Farmington River, and other tributaries, between Northampton, Mass., and Hartford, Conn. The discharge of these tributaries was enough to make the flood peak third highest at Hartford, in a record that dates from the time of the early settlements in New England. There was inundation of the flood plains along the main channel with considerable rural damage, but damage was reduced at a number of communities because of the dikes that had been constructed after earlier floods. It is estimated that Hartford itself was saved a large amount of destruction by the flood-protective works along Park River, which reached a peak of $16,200 \mathrm{cfs}$, about 3 times as high as in 1938 .

The major damage in western Massachusetts occurred in the Westfield River basin. It was in this basin that the maximum rainfall(nearly 20 inches) for the Diane storm was measured (see fig. 3), and this cloudburst fell on the large area that had an 8 -inch rainfall in the Connie storm. Damage throughout the basin was of disaster proportions. Spectacular damage was caused by small tributaries with precipitous slopes.
Long stretches of valley roads disappeared completely. Tobacco crops and harvested leaf tobacco in the flood plains along lower Westfield River and tributaries were total losses in extensive areas. The typical assortment of flood damages occurred and reached a maximum in the industrial city of Westfield.

In the Westfield River basin the Knightville flood control dam cut off the contribution from 162 square miles (the dam had not been built in 1938). Despite this reduction in effective drainage area, the gaging station on Westfield River near Westfield, Mass., had a peak flow of $70,300 \mathrm{cfs}$ as compared with $55,500 \mathrm{cfs}$ in 1938, and the stage was 4.8 feet higher. The highest known unit discharge in the entire flood area of the northeast occurred in Powdermill Brook near Westfield, where a determination of peak discharge showed 2,300 cfs per square mile from a drainage area of 2.50 square miles.

Farmington River basin was one of the most seriously affected areas in the flood. West Branch Farmington River heads in Massachusetts, flows southward into west-central Connecticut, and empties into Connecticut River not far above Hartford. The main stem and all tributaries of Farmington River had flood flows of disaster proportions. Winsted, Conn., the scene of some of the most spectacular damage, is in this basin. Here, Mad River overflowed its constricted channel and destroyed much of the commercial and industrial part of the city. The flow of Mad River near Winsted was computed to be $10,200 \mathrm{cfs}$ from a drainage area of 19.7 square miles. The combination of high rainfall and a narrow, populated valley caused a large amount of damage, and a number of deaths occurred.

Rates of discharge were very high in the upper tributaries of Farmington River. In the vicinity of Barkhamsted Reservoir, Valley Brook near West Hartland, Conn., had a unit peak discharge of 1,150 cfs per square mile, from a drainage area of 7.20 square miles; East Branch Salmon Brook at North Granby, Conn., had a unit discharge of $1,080 \mathrm{cfs}$ per square mile from 13.2 square miles.

Naugatuck River is the principal stream in westcentral Connecticut. Tributaries in the upper half of the basin and the main stem throughout had record high rates of discharge. The valley of the Naugatuck is highly industrialized and the damage of all sorts was tremendous. The roll of disaster cities is a roll of all the communities along the main valley: Torrington, Thomaston, Waterbury, Naugatuck, Seymour, Ansonia, and others.

The flow of Naugatuck River near Thomaston was $41,600 \mathrm{cfs}$ from a drainage area of 71.9 square miles; this was 4.1 times greater than the peak flow of the 1948 flood, which was the highest in the period of record starting in 1930 . The stage of 24.0 feet near Thomaston was twice that of the 1948 flood.

The same story was repeated down the entire valley. At the city of Naugatuck, Naugatuck River reached a flow of $106,000 \mathrm{cfs}$ at a stage of $25.7 \mathrm{feet}$, as compared with 28,500 cfs and 12.4 feet respectively, in the 1948 flood, the previous high in a record extending back to 1918. There is no record of floods, either measured or historical along the main stream and along most of the tributaries that compares with this tremendous flow of water. 
Although small tributaries in the headwaters of the Housatonic River basin were fairly high and those tributaries coming in from the east had destructive flows, the upper Housatonic itself did not have an outstanding flood. At Falls Village, Conn., for instance, the discharge of $22,700 \mathrm{cfs}$ was slightly lower than the previous high in the period of record starting in 1912.

The middle reaches of Housatonic River, however, had new peaks and a considerable amount of flood destruction. At Gaylords ville, Conn., the peak discharge of $51,800 \mathrm{cfs}$ was 1.6 times the peak that occurred in 1938. One of its tributaries, Tenmile River, also had a new peak discharge with about the same relation to the 1938 peak.

Shepaug River, which lies between the Housatonic and the Naugatuck, had a tremendous flood. The discharge at the gaging station near Roxbury was 50,300 cfs, as compared with $10,500 \mathrm{cfs}$ in 1938 . This new peak was at a unit rate of $378 \mathrm{cfs}$ per square mile from a drainage area of 133 square miles.

A similar relationship existed on Pomperaug River, which is tributary to Housatonic River. At Southbury, the peak discharge was $29,400 \mathrm{cfs}, 4.0$ times the 1938 peak, and the stage was about 5.8 feet higher than in 1938.

Farther downstream, at the gaging station at Stevenson, the peak discharge of Housatonic River was high, being slightly less than that in 1936 , which happened to be the year of the previous high discharge at that point.

Except for the destructive flows in the Woonsocket area, streamflow during the Diane flood was generally moderate to low in Rhode Island and in the southern part of Connecticut. The exceptions to this would be the main stems of the larger streams which carried large amounts of flood water to Long Island Sound, streams such as Thames River, Connecticut River and Housatonic River. The flood debris from these streams prevented use by boats of some harbors along the Connecticut shore. Wreckage, and even salvagable materials, floated to the Long Island shore where it was deposited on the beaches in large quantities.

\section{New York}

The area of severe flooding extended into New York, but damage was limited mostly to a narrow band from the vicinity of Poughkeepsie to Port Jervis, and in the Wallkill River basin. The usual flood damage occurred but the total in New York was the least among the six States most affected by the floods of August 18-19.

East of Hudson River, outstanding floods occurred on Wappinger Creek and Bashbish Creek. Several bridges were destroyed in the Copake Falls area where Bashbish Creek produced a peak discharge of about $10,800 \mathrm{cfs}$ from 15.8 square miles. At the gaging station on Wappinger Creek near Wappingers Falls, a new peak was established for the record started in 1928 .

An area of major flooding was the Rondout Creek and Wallkill River basins, on the west side of Hudson River. Tributaries of Rondout Creek were particularly high and considerable damage occurred in the Ellenville, N. Y. , area. A new peak discharge occurred at the gaging station on Rondout Creek at Rosendale for a record of 29 years.

Extensive areas of rich bottom lands were inundated in the Wallkill River basin, resulting in the loss of a large part of an onion crop, one of the specialties of the region. New peak discharges were recorded at six of the eight gaging stations in the basin.

Highly destructive flows occurred in all the streams tributary to Rondout Creek and Neversink River in the valley which extends from Kingston, N. Y., to Port Jervis, N. Y. At the gaging station at Godeffroy, the flood peak of Neversink River was $33,000 \mathrm{cfs}$, about 1.6 times the previous peak. U. S. Highway 209 was inundated at many places and many small-stream bridges were destroyed.

\section{New Jersey}

Among the six States in the flood area, New Jersey was fourth in flood damage, in descending order. The principal areas of flooding were in part of the Passaic River basin, the lower part of the Raritan River basin, and the main Delaware River and its northern tributaries. Except along the Delaware, the damage was essentially scattered and no deaths were reported in New Jersey. There was a considerable amount of damage in the village of Branchville as a result of the destruction of a dam on a relatively small stream.

In north-central New Jersey, a new peak discharge was established at only one gaging station, that on Wanaque River at Monks. The peak flow at this station was $3,400 \mathrm{cfs}$, as compared with $2,660 \mathrm{cfs}$ during the previous highest flood.

New peaks were established at three gaging stations in the Raritan River basin. One was the $18,000 \mathrm{cfs}$ reading at the gaging station on South Branch Raritan River at Stanton, which was about 1.7 times the previous peak.

The most notable feature of the August floods in New Jersey was the great flood on Delaware River. At all five gaging stations between Port Jervis, N. Y. , and Trenton, N. J., new peaks were established, both in stage and discharge, exceeding even the historical flood of 1903. Many communities were damaged extensively by both quiet inundation and fast-moving water. Three bridges across Delaware River were destroyed: the bridge between Easton, Pa., and Phillipsburg, N. J.; the old bridge between Portland, $\mathrm{Pa}$. , and Columbia, N. J.; and the bridge between Point Pleasant, Pa., and Byram, N. J. It is noteworthy that the old covered bridge at Portland and Columbia was started in 1831 and was completed in 1869. It withstood all floods until the disaster in August 1955, and even then it gave way only when it was about three-quarters submerged.

At Port Jervis, N. Y., the peak stage of Delaware River was about 0.8 feet higher than in October 1903 and the discharge was computed to be 14 percent higher. At the last gaging station downstream, at Trenton, the stage was 0.1 feet higher than in 1903 . A stage 2 feet higher occurred in 1904 as a result of an ice jam. The memories of old residents and the historical records indicate that in discharge the 1955 flood was the greatest on Delaware River since the country was first settled. 
Pennsylvania

The area of severe flooding in Pennsylvania during the floods of August 18-19, 1955, extended from Lackawanna, Susquehanna, and Schuylkill Rivers to Delaware River. A large part of the damage occurred in the Pocono Niountains region, but a considerable amount occurred elsewhere.

Flood flows were particularly high in the Lackawaxen River basin. The peak flow of East Branch Wallenpaupack Creek at Greentown, $\mathrm{Pa}$., was $33,300 \mathrm{cfs}$ from 33.9 square miles, which gives a unit discharge of 982 cfs per square mile. Ten people died at Greentown in the portion of the community that was on the flood plain.

For the first time in many years, and one of the few times that it has occurred, Wallenpaupack Lake overflowed, but discharge from the lake did not begin until after the peak had passed in Lackawaxen River, into which the lake drains.

The village of Hawley, Pa., was severly damaged. One of the outstanding areas of destruction for the entire flood was at Newfoundland, $\mathrm{Pa}$., where essentially the entire community was swept by the waters from Wallenpaupack Creek. The dreadful monotony of such destruction is indescribable.

All small and large tributaries (in Pennsylvania) of Delaware River from Port Jervis to Delaware Water Gap, had very high and very destructive flows. Practically every bridge along U. S. Highway 209 in that reach was destroyed or heavily damaged. The flow of Bush Kill at Shoemakers, $\mathrm{Pa}$., reached a peak of $23,400 \mathrm{cfs}$, which was 4.5 times the previous peak (1920) in the period of record starting in 1908.

The most spectacular phase of the floods in Pennsylvania occurred in the Brodhead Creek basin, the upper part of which is in the Pocono Mountains. Flood flow in all parts of the basin was highly destructive and damage of all kinds occurred from Canadensis to Minisink Hills. The scene of the greatest single tragedy of the August floods, was just a few miles above Stroudsburg, $\mathrm{Pa}$., where more than 30 persons, mostly children, lost their lives at a camp on the banks of Brodhead Creek. The main line of the Delaware, Lackawanna, \& Western Railroad was destroyed at a number of places and service was not resumed for about two months. Damage to the railroad amounted to millions of dollars.

At Analomink, Pa. , the peak flow of Brodhead Creek was computed to be $72,200 \mathrm{cfs}$ from a drainage area of 124 square miles, giving a unit discharge of $582 \mathrm{cfs}$ per square mile. This was a far greater flood flow than any before, even back into the historical records.

The flood in the Lehigh River basin was a major flood. It was principally an upper basin flood but new records were established at all gaging stations along the main stem of the Lehigh except the one farthest downstream, at Bethlehem, $\mathrm{Pa}$. A flow of $58,300 \mathrm{cfs}$ was computed at Tannery, Pa. This rate of discharge was about twice that previously recorded in the 41 years of record at the station, and the stage was 5.7 feet higher.

Similar high flows occurred in other important tributaries of Delaware River; for instance, Neshaminy
Creek near Langhorne, Pa., had a peak of $49,300 \mathrm{cfs}$, about twice the previous high in the 20 years of record.

The northern tributaries of Schuylkill River contributed large amounts of runoff and a considerable amount of damage occurred. For the Schuylkill, it was mostly a tributary flood, but a new high was recorded on the main stem at Berne, $\mathrm{Pa}$. The damage area extended into the city of Philadelphia although the destruction there was not great.

In the Susquehanna River basin, most of the heavy damage was concentrated in the Scranton and WilkesBarre area, caused mostly by small streams. One of the outstanding flows occurred on Roaring Brook at Dunmore, $\mathrm{Pa}$., where the peak discharge of $18,500 \mathrm{cfs}$ gave a unit rate of $374 \mathrm{cfs}$ per square mile from 49.4 square miles.

Damaging flows occurred in tributaries of Susquehanna River as far south as Bloomsburg, but the rise on the Susquehanna itself was only relatively moderate.

\section{Summary of Flood Damage}

The damage caused by the floods of August 18-19 in the northeastern States has been estimated to be in the magnitude of half a billion dollars. Early estimates ran six times as high, but they were made at a time when the damage could not be assessed accurately. The estimates of flood damages vary with different methods of evaluation, particularly with respect to intangible damage.

The Corps of Engineers is making detailed damage surveys but these were not available for inclusion in this report. A summary prepared by the Business and Defense Services Administration as listed in their press release 2 / is presented in table 1 . The figures in table 1 are subject to modification as the full story of the flood damage is revealed.

Data are not available at the moment on the number of highway bridges destroyed or on many other specific items.

\section{FLOOD DATA}

Data on the floods of August 1955 in the northeastern States were collected at many gaging stations operated by the U. S. Geological Survey in the six-State area. The basic records are continuous records of stage and discharge, which may be combined for varying periods and in various ways to suit the needs of the user. To supplement the records for the gaging stations, peak discharge also was determined at many miscellaneous sites, where there were no gaging stations. The location of the gaging stations and the miscellaneous sites are shown on plate 1

Stage and discharge data have been assembled in the following sections of this report. Data for selected gaging stations are presented as it is not possible to show in this preliminary report the records for all

2/Press release BD-574, Business and Defense Services Administration, U. S. Dept. of Commerce, Washington, D. C., October 31, 1955. 
Table 1. --Summary of damages and casualties caused by floods of August 18-19, 1955, in the Northeast.

[Data provided by Business and Defense Services Administration, U. S. Dept. of Commerce]

\begin{tabular}{|c|c|c|c|c|c|c|c|}
\hline Classification & Massachusetts & Rhode Island & Connecticut & New York & New Jersey & Pennsylvania & Total \\
\hline Industrial - - & $\$ 55,000,000$ & $\$ 5,700,000$ & $\$ 83,871,000$ & $\$ 382,000$ & $\$ 1,250,000$ & a $20,684,000$ & $\begin{array}{r}\$ 146,203,000 \\
20,684,000\end{array}$ \\
\hline Commercial -- & $5,410,000$ & $2,200,000$ & $42,601,000$ & $1,354,000$ & 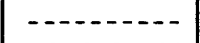 & $\ldots \ldots$ & $51,565,000$ \\
\hline Roads - & $23,476,000$ & 900,000 & $26,233,000$ & $6,380,000$ & $8,500,000$ & $16,937,000$ & $82,426,000$ \\
\hline Dwellings - - & b 198,000 & $3,000,000$ & $23,953,000$ & 968,000 & $12,954,000$ & $6,034,000$ & $47,107,000$ \\
\hline Utilities - - & $25,000,000$ & $6,200,000$ & $29,702,000$ & $5,000,000$ & $3,986,000$ & $9,419,000$ & $79,307,000$ \\
\hline Agricultural -- & $1,329,000$ & $\ldots \ldots$ & $2,424,000$ & $2,200,000$ & 10,000 & $1,082,000$ & $7,045,000$ \\
\hline Miscellaneous- & 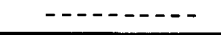 & 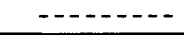 & $6,461,000$ & $-\ldots$ & c 825,000 & $\mathrm{~d} 16,051,000$ & $23,337,000$ \\
\hline Total-_..-.-- & $\$ 110,413,000$ & $\$ 18,000,000$ & $\$ 215,245,000$ & $\$ 16,284,000$ & $\$ 27,525,000$ & $\$ 70,207,000$ & $\$ 457,674,000$ \\
\hline $\begin{array}{l}\text { Dwellings } \\
\text { destroyed -.. }\end{array}$ & 97 & 34 & 563 & 26 & 93 & 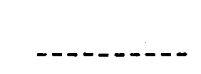 & 813 \\
\hline \multicolumn{8}{|c|}{ Casualties } \\
\hline Deaths & 12 & 1 & 77 & 1 & 0 & 88 & 179 \\
\hline Major injuries & 77 & 32 & 6,773 & 6 & 10 & 94 & 6,992 \\
\hline
\end{tabular}

a Industrial and commercial.

b Appears to be low.

c Railroad damage.

d Includes $\$ 14,618,000$ railroad damage.

gaging stations in the flood area. Graphs of data are presented to show the interrelation of the flows at the many points where discharge was determined, but comprehensive analyses were not made.

The problem of obtaining records of stage and discharge at some of the gaging stations was made more difficult by the destruction of part or all of the station facilities. The floods destroyed gage houses, cableways, controls, and associated parts of station installations. At several places, the recorders were swept away.

\section{Measurement of Flood Discharges}

Whenever possible, the discharge of streams at gaging stations is measured by current meter, at both high or low stages. Current-meter measurements were made at some of the gaging stations during the August floods. It was impossible to reach them all at or near the peak stage, because of road conditions and because of the number of engineers available.

At many gaging stations, and at all miscellaneous sites, the peak discharge during the floods was determined by what are known as indirect methods. Such determinations are made by one or more of the following methods: Slope area, contraction, culvert flow, flow over dam, and flow over embankment.

The field work associated with the indirect determinations was accomplished after the August floods had subsided. Using the methods listed above, it was possible to obtain a wealth of peak-flow information at many places where the data could not have been obtained by other means.

\section{Summary of Stages and Discharges}

A summary of stages and discharges that occurred in the August floods has been prepared, presented on the succeeding pages as table 2 . The summary lists the gaging stations and miscellaneous sites in downstreamorder. The reference numbers refer to the sites as identified on the general map, plate 1 .

For gaging stations, the highest flood in the period of record is shown. At some stations, the highest stage and highest discharge may not have occurred during the same flood. Any greater flood outside the period of record is listed if known.

At points where peak-discharge was not determined by gaging-station operations, a headnote is used to show the method of determination. 
Table 2. --Summary of stages and discharges in the Northeast during the floods of August 1955

[Station description and hydrograph data are given in this report for the gaging stations indicated by an asterisk (*). Maximum discharges for the floods of August 1955 were obtained from gaging station records or by special methods as indicated by the following symbols: A, slope-area measurement; B, contracted-opening measurements; C, computation of flow through culvert; $D$, computation of flow over dam; E, computation of flow over embankment]

\begin{tabular}{|c|c|c|c|c|c|c|c|c|c|c|c|}
\hline \multirow{2}{*}{$\begin{array}{l}\text { No. } \\
\text { on } \\
\text { pl } 1\end{array}$} & \multirow[b]{2}{*}{ Stream and place of determination } & \multirow{2}{*}{$\begin{array}{c}\text { Drainage } \\
\text { area } \\
\text { (sq } \mathrm{mi})\end{array}$} & \multirow{2}{*}{$\begin{array}{l}\text { Period } \\
\text { of } \\
\text { record }\end{array}$} & \multicolumn{4}{|c|}{ Maximum flood previously known } & \multicolumn{4}{|c|}{ Maximum during present flood } \\
\hline & & & & Date & $\begin{array}{l}\text { Gage } \\
\text { heijht } \\
\text { (feet) }\end{array}$ & $\begin{array}{l}\text { Discharge } \\
\text { (cfs) }\end{array}$ & $\begin{array}{l}\text { Cfs per } \\
\text { sq mi }\end{array}$ & Date and hour & $\begin{array}{l}\text { Gage } \\
\text { hei yhit } \\
\text { (feet) }\end{array}$ & \begin{tabular}{|c|}
$\begin{array}{c}\text { Discharge } \\
\text { (cfs) }\end{array}$ \\
\end{tabular} & $\begin{array}{c}\text { Cfs per } \\
\text { sq mi }\end{array}$ \\
\hline & MERRIMACK RIVER BASIN & & & & & & & & & & \\
\hline 1 & Assabet River at Maynard, Mass. & 116 & $1941-55$ & Sept. 13, 1954 & 6.47 & 1,870 & 16.1 & Aug. 20, 7:30 to 8 p.m. & 8.96 & 4,270 & 36.8 \\
\hline $2 *$ & $\begin{array}{c}\text { Concord River below River Meadow } \\
\text { Brook, at Lowell, Mass. } \\
\text { CHARLES RIVER BASIN }\end{array}$ & 405 & $1936-55$ & July 29,1938 & 8.11 & 3,790 & a 12.1 & Aug. 23,8 to 10 p. m. & 8.97 & 4,540 & a 14.6 \\
\hline $3 *$ & $\begin{array}{l}\text { Charles River at Charles River } \\
\text { Village, Mass. }\end{array}$ & 184 & $1937-55$ & $\begin{array}{l}\text { July } 27,1938 \\
\text { March }\end{array}$ & 9.00 & $\begin{array}{l}3,110 \\
3,170\end{array}$ & $\begin{array}{l}16.9 \\
17.2\end{array}$ & Aug. 23,2 to 3 p. m. & 9.24 & 3,220 & 17.5 \\
\hline 4 & Mother Brook at Dedham, Mass. & - & $1931-55$ & July $28,29,1938$ & 91.84 & 909 & - & Aug. 24,5 to 8 a. $\mathrm{m}$. & 92.90 & 960 & - \\
\hline 5 & $\begin{array}{r}\text { Charles River at Waltham, Mass, } \\
\text { NEPONSET RIVER BASIN }\end{array}$ & 251 & $1931-55$ & Mar. 19, 1936 & 4.79 & 2,540 & 10.1 & Aug. $19,3: 30$ p. m. & 5.35 & 2,490 & 9.92 \\
\hline 6 & $\begin{array}{c}\text { Neponset River at Norwood, Mass. } \\
\text { TAUNTON RIVER BASIN }\end{array}$ & 35.2 & $1939-55$ & $\begin{array}{lr}\text { May } & 9,1954 \\
\text { July } & 24,1938\end{array}$ & $\begin{array}{r}10.90 \\
b 11.05\end{array}$ & 430 & 12.2 & Aug. $19,6: 30$ p. m. & $\mathrm{b} 14.65$ & 1,450 & 41.2 \\
\hline 7 & Taunton River at State Farm, Mass. & 260 & $1929-55$ & $\begin{array}{l}\text { Dec. } 8,1945 \\
\text { May } 18,1954\end{array}$ & 11.57 & 3,190 & 12.3 & Aug. 22,7 to 8 p. m. & 13.02 & 4,280 & 16.5 \\
\hline 8 & Wading River at West Mansfield, Mass. & 19.2 & $1953-55$ & May $9,10,1954$ & 4.89 & 188 & $9.7 \mathrm{~s}$ & Aug. $20,4: 30$ to $6: 30$ p.m & 6.22 & 517 & 26.9 \\
\hline 9 & $\begin{array}{r}\text { Wading River near Norton, Mass. } \\
\text { PROVIDENCE RIVER BASIN }\end{array}$ & 42.4 & $1925-55$ & Mar. 12,13,1936 & 10.01 & 1,030 & 24. 3 & Aug. 20,7 a. m. & 10.98 & 1,170 & 27.6 \\
\hline 10 & Kettle Brook at Worcester, Mass. & 31.3 & $1923-55$ & Mar. 18, 1936 & b 8.58 & 2,520 & 80.5 & Aug. $19,12 \mathrm{~m}$. & b 12.78 & $3,970 \mathrm{D}$ & 127 \\
\hline 11 & $\begin{array}{l}\text { Quinsigamond River at North Grafton, } \\
\text { Mass. }\end{array}$ & 25.5 & $1939-55$ & Sept. 12, 1954 & 3.50 & 395 & 15.5 & Aug. $20,12 \mathrm{~m}$. & 5.15 & 840 & 32.9 \\
\hline 13 & Mumford River at East Douglas, Mass. & 27.8 & $1939-51$ & Mar. 22, 1948 & 5.10 & 420 & 15.1 & Aug. 19 & b 12.0 & $2,140 \mathrm{D}$ & 77.0 \\
\hline 14 & Blackstone River at Blackstone, Mass. & 259 & - & March 1936 & - & 11,800 & 45.6 & Aug. 19 & - & $18,800 \mathrm{D}$ & 72.6 \\
\hline
\end{tabular}




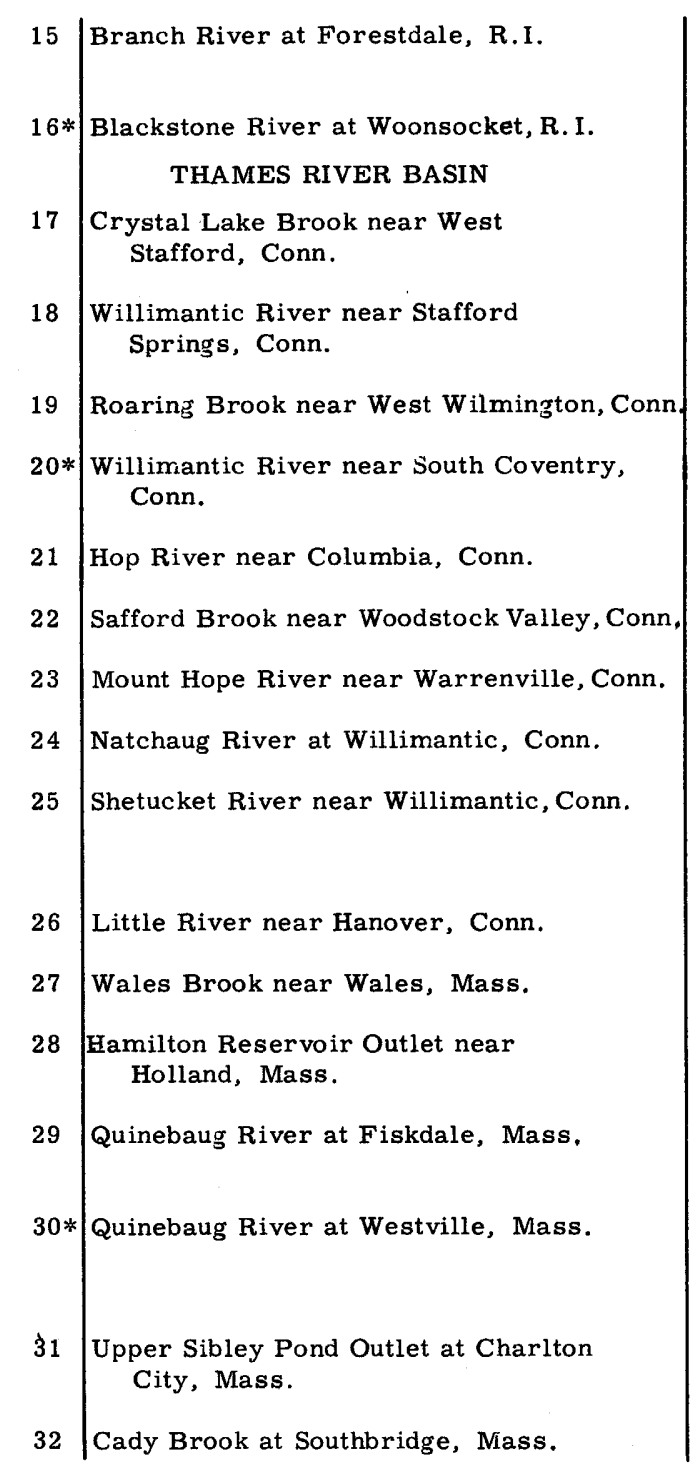
See footnotes at end of table, p. 29.

\begin{tabular}{|c|c|c|c|}
\hline 93.3 & $|1940-55|$ & $\left|\begin{array}{ll}\text { Sept. 12, } & 1954 \\
\text { March } & 1936\end{array}\right|$ & $\begin{array}{c}9.12 \\
-\end{array}$ \\
\hline 416 & $1929-55$ & July 24,1938 & 14.43 \\
\hline 5.83 & -- & $-\cdot-$ & - \\
\hline 53.5 & -- & -- & - \\
\hline 18.0 & --- & $\cdots$ & - \\
\hline 121 & $1931-55$ & Sept. 21, 1938 & 18.08 \\
\hline 76.2 & $1932-55$ & Sept. 21, 1938 & 16.25 \\
\hline 4.08 & $1950-55$ & Sept. 11, 1954 & 5.89 \\
\hline 29.1 & $1940-55$ & Sept. 11, 1954 & 9.20 \\
\hline 169 & $1930-55$ & Sept. 21, 1938 & 16.39 \\
\hline 401 & $\begin{array}{l}1904-5 \\
1919-21 \\
1928-55\end{array}$ & Sept. 21, 1938 & 27.6 \\
\hline 29.8 & $1951-55$ & Sept. 12, 1954 & 5.32 \\
\hline 3.71 & --- & --- & - \\
\hline 24.2 & --- & -- & - \\
\hline 67.5 & -- & $\begin{array}{c}\text { September } \\
1938\end{array}$ & - \\
\hline 93.8 & $1939-55$ & \begin{tabular}{|} 
Mar.22, 1948 \\
September \\
1938
\end{tabular} & $\begin{array}{c}6.93 \\
-\end{array}$ \\
\hline 2.23 & --- & --- & - \\
\hline 12.0 & -- & --- & - \\
\hline
\end{tabular}

\begin{tabular}{|c|c|c|}
\hline 10.52 & 4,240 & 45.4 \\
\hline 21.8 & $\mathrm{c} .32,900 \mathrm{~A}$ & - \\
\hline - & $784 \mathrm{~A}$ & 134 \\
\hline - & $14,500 \mathrm{~A}$ & 271 \\
\hline- & $2,920 \mathrm{C}$ & 162 \\
\hline 18.66 & $24,200 \mathrm{~B}$ & 200 \\
\hline b 15.10 & 4,620 & 60.6 \\
\hline 6.68 & $1,000 \mathrm{CE}$ & 245 \\
\hline 10.41 & $5,590 \mathrm{~B}$ & 192 \\
\hline 5.68 & $\mathrm{~d} 1,470$ & d 147 \\
\hline 17.36 & $\mathrm{~d} 21,300$ & d 88.0 \\
\hline 6.48 & 1,400 & 47.0 \\
\hline - & $1,080 \mathrm{~A}$ & 291 \\
\hline - & $6,540 B$ & 270 \\
\hline- & $15,400 \mathrm{~A}$ & \\
\hline 16.11 & $17,500 \mathrm{~A}$ & 187 \\
\hline - & $1,240 \mathrm{DE}$ & 556 \\
\hline & If $26,300 A$ & - \\
\hline
\end{tabular}




\begin{tabular}{|c|c|c|c|c|c|c|c|c|c|c|c|}
\hline \multirow[b]{2}{*}{$\begin{array}{c}\text { No. } \\
\text { on } \\
\text { pl. } \\
1 \\
\end{array}$} & \multirow[b]{2}{*}{ Stream and place of determination } & \multirow[b]{2}{*}{$\begin{array}{c}\text { Drainage } \\
\text { area } \\
\text { (sq } \mathrm{mi})\end{array}$} & \multirow[b]{2}{*}{$\begin{array}{l}\text { Period } \\
\text { of } \\
\text { record }\end{array}$} & \multicolumn{4}{|c|}{ Maximum f̂lood previously known } & \multicolumn{4}{|c|}{ Maximum during present flood } \\
\hline & & & & Date & $\begin{array}{l}\text { Gage } \\
\text { hei-int } \\
\text { (iet) }\end{array}$ & $\begin{array}{l}\text { Discharge } \\
\text { (cfs) }\end{array}$ & $\begin{array}{l}\text { Cfs per } \\
\text { sq mi }\end{array}$ & Date and hour & $\begin{array}{l}\text { Gage } \\
\text { height } \\
\text { (feet) }\end{array}$ & $\begin{array}{c}\text { Discharge } \\
\text { (cfs) }\end{array}$ & $\begin{array}{l}\text { Cfs per } \\
\text { sq mi }\end{array}$ \\
\hline 33 & $\begin{array}{l}\text { THAMES RIVER BASIN--Continued } \\
\text { Cohasse Brook at Southbridge, Mass. }\end{array}$ & 2.87 & $\cdots$ & $-\cdot$ & - & - & - & Aug. 19 & - & $1,280 \mathrm{D}$ & 446 \\
\hline 34 & Lebanon Brook at Southbridge, Mass. & 10.0 & -- & -- & - & - & - & Aug. 19 & - & $1,140 \mathrm{D}$ & 114 \\
\hline 35 & $\begin{array}{l}\text { Alder Meadow Brook near Spencer, } \\
\text { Mass. }\end{array}$ & 2.18 & --- & -- & - & - & - & Aug. 19 & - & $1,530 \mathrm{~A}$ & 702 \\
\hline 36 & French River at North Oxford, Mass. & 24.1 & -- & Mar. 18, 1936 & - & 2,030 & 84.2 & Aug. 19 & - & $8,540 \mathrm{AD}$ & 354 \\
\hline 37 & $\begin{array}{l}\text { South Fork Little River at outlet of } \\
\text { Granite Reservoir at South Charlton, } \\
\text { Mass. }\end{array}$ & 7.97 & $\cdots$ & --- & - & - & - & Aug. 19 & - & $2,060 \mathrm{DE}$ & 258 \\
\hline 38 & Little River at Buffumville, Mass, & 27.7 & $1939-55$ & Sept. 12, 1954 & 7.33 & 1,220 & 44.0 & Aug. $19,4: 30$ p. m. & 15.53 & $8,340 \mathrm{D}$ & 301 \\
\hline $39 *$ & French River at Webster, Mass. & 85.3 & $1948-55$ & $\begin{array}{l}\text { Sept. 12, } 1954 \\
\text { Mar. 19, } 1936\end{array}$ & 11.64 & $\begin{array}{l}2,320 \\
4,700\end{array}$ & $\begin{array}{l}27.2 \\
55.1\end{array}$ & Aug. 19,6 p. m. & 26.05 & $14,400 \mathrm{D}$ & 169 \\
\hline 40 & Quinebaug River at Quinebaug, Conn. & 157 & $1931-55$ & Sept. 21, 1938 & 16.21 & 14,100 & 89.8 & Aug. 19 & b 18.96 & $49,300 \mathrm{~A}$ & 314 \\
\hline 41 & Quinebaug River at Putnam, Conn. & 331 & $1929-55$ & Sept. 21, 1938 & 19.45 & 20,900 & 63. 1 & Aug. 19 & $\mathrm{~b} 26.5$ & $48,000 \mathrm{~A}$ & 145 \\
\hline 42 & Five Mile River at Killingley, Conn. & 58.2 & $1937-55$ & July 24, 1938 & 8.52 & 2,480 & 42.6 & Aug. 20, 4 p. m. & 5.76 & 1,120 & 19.2 \\
\hline 43 & $\begin{array}{l}\text { Quinebaug River at Dyer Dam site near } \\
\text { Danielson, Conn. }\end{array}$ & 465 & $-\cdots$ & Mar. 19, 1936 & - & 22,800 & 49.0 & About Aug. 19 & - & $43,900 \mathrm{~B}$ & 94.4 \\
\hline 44 & Moosup River at Moosup, Conn. & 83.5 & $1932-55$ & Mar. 12, 1936 & 8.35 & 4,260 & 51.0 & Aug. 20,7 a. $\mathrm{m}$. & 5.17 & 1,520 & 18.2 \\
\hline $45 *$ & Quinebaug River at Jewett City, Conn. & 711 & $1918-55$ & Mar. 19, 1936 & 24.0 & 29,200 & 41.1 & Aug. 20, 5-6 p. m. & 29.0 & $40,700 \mathrm{D}$ & 57.2 \\
\hline 46 & $\begin{array}{l}\text { Yantic River at Yantic, Conn. } \\
\text { CONNECTICUT RIVER BASIN }\end{array}$ & 88.6 & $1930-55$ & Sept. 21, 1938 & 14.66 & 13,500 & 152 & Aug. $19,11: 30$ p. m. & 7.53 & 1,950 & 22.0 \\
\hline $47 *$ & $\begin{array}{l}\text { Connecticut River at Montague City, } \\
\text { Mass. }\end{array}$ & 7,865 & $1904-55$ & Mar. 19, 1936 & 49.2 & 236,000 & 30.0 & Aug. 14,1 to $1: 30$ p.m. & 19.75 & 42,800 & 5.44 \\
\hline 48 & Mill River at Northampton, Mass. & 52.8 & $|1938-55|$ & $\|$ Mar. 31, 1951 & 9.38 & 3,840 & 72.7 & Aug. 19,7 a. $\mathrm{m}$. & 11.78 & $6,300 \mathrm{D}$ & 119 \\
\hline
\end{tabular}



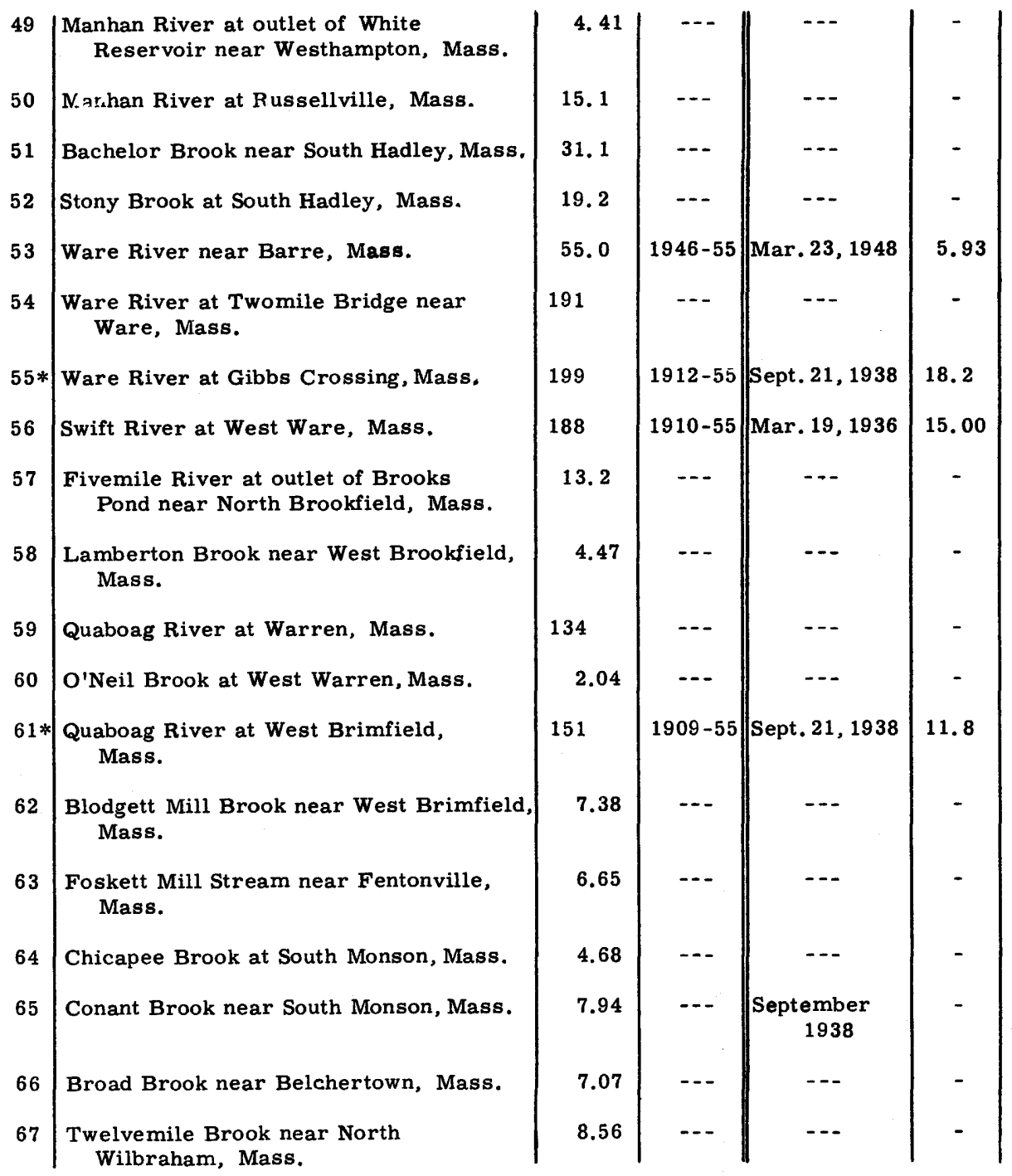

\begin{tabular}{|c|c|c|}
\hline- & - & Aug. 19 \\
\hline - & & Aug. 19 \\
\hline - & - & Aug. 19 \\
\hline - & - & Aug. 19 \\
\hline 1,450 & 26.4 & Aug. 20,8 to $8: 30$ a.m. \\
\hline- & - & Aug. 19 \\
\hline 22,700 & 114 & Aug. 19,10 a. m. \\
\hline 7,590 & 40.4 & Aug. 19 \\
\hline- & - & Aug. 19 \\
\hline - & - & Aug. 19 \\
\hline - & - & Aug. 19 \\
\hline - & - & Aug. 19 \\
\hline 8,470 & 56.1 & Aug. $19,8: 30$ a. m. \\
\hline - & - & Aug. 19 \\
\hline - & - & Aug. 19 \\
\hline - & - & Aug. 19 \\
\hline 1,230 & 155 & Aug. 19 \\
\hline - & - & Aug. 19 \\
\hline - & - & Aug. 19 \\
\hline
\end{tabular}

\begin{tabular}{|c|c|c|}
\hline - & 1,080 & 245 \\
\hline- & g9, 350A & 619 \\
\hline - & $z 5,320 \mathrm{~B}$ & 171 \\
\hline - & $1,920 \mathrm{D}$ & 100 \\
\hline 5.53 & 1,120 & 20. \\
\hline - & $9,530 \mathrm{BE}$ & 49. \\
\hline 12.83 & $12,200 \mathrm{~A}$ & 61. \\
\hline 5.68 & h 955 & - \\
\hline - & $1,730 \mathrm{D}$ & 131 \\
\hline - & $4,140 \mathrm{~A}$ & 926 \\
\hline - & $\mathrm{g} \mathrm{4,730 \textrm {B }}$ & 35 \\
\hline - & $1,670 \mathrm{~A}$ & 819 \\
\hline 14.79 & $12,800 \mathrm{~A}$ & 84 \\
\hline - & $5,860 \mathrm{~A}$ & 794 \\
\hline - & g 3, 900D & 586 \\
\hline - & 1,700 & 363 \\
\hline - & $5,600 \mathrm{D}$ & 705 \\
\hline & $4,740 \mathrm{~A}$ & 670 \\
\hline & g $8,690 \mathrm{~A}$ & \\
\hline
\end{tabular}

See footnotes at end of table, p. 29 . 


\begin{tabular}{|c|c|c|c|c|c|c|c|c|c|c|c|}
\hline \multirow[b]{2}{*}{$\begin{array}{c}\text { No. } \\
\text { on } \\
\text { pl. } \\
1\end{array}$} & \multirow[b]{2}{*}{ Stream and place of determination } & \multirow[b]{2}{*}{$\begin{array}{c}\text { Drainage } \\
\text { area } \\
\text { (sq mi) }\end{array}$} & \multirow[b]{2}{*}{$\begin{array}{c}\text { Period } \\
\text { of } \\
\text { record }\end{array}$} & \multicolumn{4}{|c|}{ Maximum flood previously known } & \multicolumn{4}{|c|}{ Maximum during present flood } \\
\hline & & & & Date & $\begin{array}{c}\text { Gage } \\
\text { height } \\
\text { (feet) }\end{array}$ & $\begin{array}{c}\text { Discharge } \\
\text { (cfs) }\end{array}$ & $\begin{array}{l}\text { Cfs per } \\
\text { sq mi }\end{array}$ & Date and hour & $\begin{array}{c}\text { Gage } \\
\text { height } \\
\text { (feet) }\end{array}$ & $\begin{array}{c}\text { Discharge } \\
\text { (cfs) }\end{array}$ & $\begin{array}{l}\text { Cfs per } \\
\text { sq mi }\end{array}$ \\
\hline $68 *$ & $\begin{array}{l}\text { CONNECTICUT RIVER BASIN--Con. } \\
\text { Chicopee River at Indian Orchard, } \\
\text { Mass. }\end{array}$ & 688 & $1928-55$ & Sept. 21, 1938 & - & 45,200 & 65.7 & Aug. 19,4 p.m. & 22.14 & h 40,500D & h 80.7 \\
\hline 69 & Mill River at Springfield, Mass. & 33.9 & $1938-51$ & --- & - & - & - & $\begin{array}{l}\text { Aug. } 19 \text {, several hours } \\
\text { in morning }\end{array}$ & $\mathrm{b} 12.02$ & 1,960 & 57.8 \\
\hline 70 & Westfield River at Knightville, Mass. & 162 & $1909-55$ & Sept. 21, 1938 & bi29.58 & 37,900 & 234 & Aug. 28,10 a. m. & 6.67 & j 4,680 & - \\
\hline $71 *$ & $\begin{array}{l}\text { Middle Branch Westfield River at } \\
\text { Goss Heights, Mass. }\end{array}$ & 52.6 & $1910-55$ & $\begin{array}{l}\text { Mar. 12, 1936 } \\
\text { Sept. 21, 1938 }\end{array}$ & $\begin{array}{l}13.87 \\
10.61\end{array}$ & 19,900 & $378^{-}$ & Aug. 19,4 a. m. & 11.33 & $16,500 \mathrm{~A}$ & 314 \\
\hline 72 & Walker Brook at Chester, Mass. & 17. 7 & $-\cdot$ & -- & - & - & - & Aug. 19 & - & $5,220 \mathrm{~A}$ & 295 \\
\hline $73 *$ & $\begin{array}{l}\text { West Branch Westfield River at } \\
\text { Huntington, Mass. }\end{array}$ & 93.7 & $1935-55$ & Sept. 21, 1938 & 15.5 & 21,800 & 233 & Aug. $19,4: 30$ a. m. & 15.27 & $26,100 \mathrm{~A}$ & 279 \\
\hline 74 & Stage Brook near Russell, Mass, & 5.21 & $-\cdots$ & $-\cdot$ & - & - & - & Aug. 19 & - & $4,910 \mathrm{~A}$ & 942 \\
\hline 75 & Black Brouk near Russell, Mass. & 2.97 & -- & --- & - & - & - & Aug. 19 & - & $1,730 \mathrm{D}$ & 593 \\
\hline 76 & $\begin{array}{l}\text { Potash Brook at Blandford-Russell } \\
\text { town line, Mass. }\end{array}$ & 1.53 & -- & ... & - & - & - & Aug. 19 & - & $1,210 \mathrm{~A}$ & 791 \\
\hline 77 & Westfield River at Woronoco, Mass. & 351 & -- & -- & - & - & - & Aug. 19 & - & $\mathrm{m} 61,500 \mathrm{D}$ & m325 \\
\hline 78 & $\begin{array}{l}\text { Cobble Mountain Reservoir tributary } \\
\text { near Granville, Mass. }\end{array}$ & .66 & --- & $+\cdots$ & - & - & - & Aug. 19 & - & $415 \mathrm{C}$ & 629 \\
\hline 79 & Cooks Brook at West Parish, Mass. & .32 & --- & --- & - & - & - & Aug. 19 & - & $218 \mathrm{CE}$ & 681 \\
\hline 80 & Dickinson Brook near Granville, Mass. & 6.42 & -- & --- & - & - & - & Aug. 19 & - & $5,750 \mathrm{~A}$ & 896 \\
\hline 81 & $\begin{array}{l}\text { Westfield Little River at Stevens } \\
\text { Paper Co. dam, at Westfield, Mass. }\end{array}$ & 77.7 & $-\cdot-$ & --- & - & - & - & Aug. 19 & - & n 21,700D & - \\
\hline 82 & Powdermill Brook near Westfield, Mass & 2.50 & --- & --- & - & - & - & Aug. 19, about 1 a.m. & - & $5,740 \mathrm{CE}$ & 2,300 \\
\hline
\end{tabular}


83 Great Brook at Southwick, Mass. 84* Westfield River near Westfield, Mass. 85* Connecticut River at Thompsonville, Conn.

86 Clay Brook near Suffield, Conn.

87 Stony Brook near Suffield, Conn.

88 Scantic River at Scitico, Conn.

89* Scantic River at Broad Brook, Conn.

90 Fall River at Otis Reservoir Outlet near Cold Spring, Mass.

91 Silver Brook at West New Boston, Mass.

92 Clam River at West New Boston, Mass, 93* West Branch Farmington River near New Boston, Mass.

94 West Branch Farmington River near Riverton, Conn.

95 Mad River near Winsted, Conn.

96 Colebrook Brook near Winsted, Conn.

97 Highland Lake outflow at Winsted, Conn.

98 Sandy Brook near Robertsville, Conn. $99 *$ Still River at Robertsville, Conn.

100 West Branch Farmington River at Riverton, Conn.

101 Morgan Brook near Winsted, Conn.

102 Hubbard River near West Hartland, Conn.

See footnotes at end of table p. 29.

\begin{tabular}{|c|c|c|c|c|c|}
\hline 19.3 & -- & -- & - & - & - \\
\hline 497 & $1914-55$ & Sept .21,22,1938 & 29.40 & 55,500 & 112 \\
\hline 661 & $1928-55$ & Mar. 20, 1936 & 16.6 & 282,000 & 29.2 \\
\hline .69 & ..- & -- & - & - & - \\
\hline 36.9 & --- & -.- & - & - & - \\
\hline 66.0 & -. & -.. & - & - & - \\
\hline 98.4 & $1928-55$ & Sept. 21, 1938 & 16.08 & 7,360 & 74.8 \\
\hline 17.2 & -- &.-- & - & - & - \\
\hline 6.52 &.-- & -- & - & - & - \\
\hline 29.3 & --- & -- & - & - & - \\
\hline 92.0 & $1913-55$ & Sept.21, 1938 & 12.94 & g 18,500 & $\lg 247$ \\
\hline 128 & $\ldots$ & ... & - & - & - \\
\hline 19.7 & -- & $\ldots$ & - & - & - \\
\hline 2.84 &.- & -- & - & - & - \\
\hline 7. 30 & $\cdots$ & --- & - & - & - \\
\hline 31.1 & -- &.- & - & - & - \\
\hline 84.4 & $1948-55$ & Dec. 31,1948 & 10.12 & 9,550 & 113 \\
\hline 216 & $1929-55$ & Sept. 21, 1938 & 17.95 & 37,100 & 172 \\
\hline 6.39 & -- & -- & - & - & - \\
\hline 19.9 & & -- & - & - & - \\
\hline
\end{tabular}

\begin{tabular}{|c|c|}
\hline - & $\circ 3,610 \mathrm{CE}$ \\
\hline 34.2 & $\mathrm{p} 70,300 \mathrm{D}$ \\
\hline 10.93 & 174,000 \\
\hline - & $531 \mathrm{C}$ \\
\hline - & $17,300 \mathrm{~A}$ \\
\hline- & $15,400 \mathrm{~B}$ \\
\hline 19.9 & $13,300 \mathrm{~A}$ \\
\hline- & 1,320 \\
\hline - & $4,370 \mathrm{~A}$ \\
\hline - & \\
\hline 14.06 & $34,300 \mathrm{~A}$ \\
\hline - & $57,200 \mathrm{~A}$ \\
\hline - & $10,200 \mathrm{~A}$ \\
\hline- & \\
\hline - & $4,050 \mathrm{AD}$ \\
\hline - & $10,100 \mathrm{~B}$ \\
\hline 16.48 & $44,000 \mathrm{~A}$ \\
\hline b20. 3 & 101,000 \\
\hline - & $2,510 \mathrm{C}$ \\
\hline - & $10,500 \mathrm{C}$ \\
\hline
\end{tabular}

187 Aug. 19, 7 to 8 a. $m$. Aug. 19, 7 p.m.

About Aug. 19

About Aug. 19

About Aug. 19

Aug. 19, 6 p.m.

Aug. 19

Aug. 19

Aug. 19

Aug. 19, 4:45 a. m.

About Aug. 19

About Aug. 19

About Aug. 19

About Aug. 19

About Aug. 19

Aug. 19, 8 a.m.

Aug. 19, 7 a.m.

About Aug. 19

About Aug. 19 


\begin{tabular}{|c|c|c|c|c|c|c|c|c|c|c|c|}
\hline \multirow{2}{*}{$\begin{array}{c}\text { No. } \\
\text { on } \\
\text { pl. } \\
1\end{array}$} & \multirow[b]{2}{*}{ Stream and place of determination } & \multirow{2}{*}{$\begin{array}{c}\text { Drainage } \\
\text { area } \\
\text { ( sq mi) }\end{array}$} & \multirow{2}{*}{$\begin{array}{l}\text { Period } \\
\text { of } \\
\text { record }\end{array}$} & \multicolumn{4}{|c|}{ Maximum flood previously known } & \multicolumn{4}{|c|}{ Maximum during present flood } \\
\hline & & & & Date & $\begin{array}{l}\text { Gage } \\
\text { height } \\
\text { (feet) }\end{array}$ & $\begin{array}{c}\text { Discharge } \\
\text { (cfs) }\end{array}$ & $\begin{array}{l}\text { Cfs per } \\
\text { sq mi }\end{array}$ & Date and hour & $\begin{array}{l}\text { Gage } \\
\text { height } \\
\text { (feet) }\end{array}$ & $\begin{array}{c}\text { Discharge } \\
\text { (cfs) }\end{array}$ & $\begin{array}{l}\text { Cfs per } \\
\text { sq mi }\end{array}$ \\
\hline 103 & $\begin{array}{l}\text { CONNECTICUT RIVER BASIN--Con. } \\
\text { Valley Brook near West Hartland, Conn. }\end{array}$ & 7.20 & $\cdots$ & -- & - & - & - & About Aug. 19 & - & $8,260 \mathrm{~A}$ & 1,150 \\
\hline 104 & Beaver Brook near Barkhamsted, Conn. & 5.31 & -- & $\cdots$ & - & - & - & About Aug. 19 & - & $3,350 \mathrm{C}$ & 631 \\
\hline 105 & $\begin{array}{l}\text { Farmington River near Collinsville, } \\
\text { Conn. }\end{array}$ & 360 & $-\cdot$ & Sept. 21, 1938 & - & 54,000 & 150 & About Aug. 19 & - & $140,000 \mathrm{~A}$ & 389 \\
\hline $106 *$ & Burlington Brook near Burlington, Conn. & 4.12 & $1931-55$ & Sept. 21, 1938 & 7.24 & 676 & 164 & Aug. 19,4 a.m. & 9.22 & $1,720 \mathrm{D}$ & 410 \\
\hline 107 & Pequabuck River at Forestville, Conn. & 45.2 & $1941-55$ & $\begin{array}{l}\text { Dec. } 31,1948 \\
\text { September } \\
1938\end{array}$ & $\begin{array}{l}6.70 \\
\text { b } 7.3\end{array}$ & $\begin{array}{l}3,260 \\
3,800\end{array}$ & $\begin{array}{l}72.1 \\
84.1\end{array}$ & Aug. $19,7: 30$ a. m. & 13.22 & $11,700 \mathrm{~A}$ & 259 \\
\hline 108 & $\begin{array}{l}\text { East Branch Salmon Brook at North } \\
\text { Granby, Conn. }\end{array}$ & 13.2 & --- & -- & - & - & - & About Aug. 19 & - & $14,300 \mathrm{~A}$ & 1,080 \\
\hline 109 & $\begin{array}{l}\text { West Branch Salmon Brook at West } \\
\text { Granby, Conn. }\end{array}$ & 11.7 & --- & --- & - & - & - & About Aug. 19 & - & $10,500 \mathrm{~A}$ & 897 \\
\hline 110 & Salmon Brook near Granby, Conn. & 60.6 & $1946-55$ & Dec. 31,1948 & 13.55 & 3,440 & 56.8 & Aug. 19,4 a. m. & 23.58 & r 40,000 & 660 \\
\hline $111 *$ & Farmington River at Rainbow, Conn. & 584 & $1928-55$ & $\begin{array}{l}\text { Sept. 22, } 1938 \\
\text { Jan. } 1,1949\end{array}$ & 13.83 & 29,900 & 51.2 & Aug. $19,10-11$ p. m. & 23.5 & $69,200 \mathrm{D}$ & 118 \\
\hline 112 & $\begin{array}{l}\text { South Branch Park River at Hartford, } \\
\text { Conn. }\end{array}$ & 40.6 & $1936-55$ & Sept. 21, 1938 & 13.6 & 3,600 & 88.7 & Aug. 19 & b 19.65 & 7,000 & 172 \\
\hline 113 & $\begin{array}{l}\text { North Branch Park River near Hartford, } \\
\text { Conn. }\end{array}$ & 18.6 & -- & -- & - & - & - & About Aug. 19 & - & $7,980 \mathrm{~A}$ & 429 \\
\hline 114 & $\begin{array}{l}\text { North Branch Park River at Hartford, } \\
\text { Conn. }\end{array}$ & 25.3 & $1936-55$ & Jan. 25, 1938 & 11.6 & 3,000 & 119 & Aug. 19 & b 18.8 & 10,000 & 395 \\
\hline 115 & Park River at Hartford, Conn. & 74.0 & $1936-55$ & Jan. 25,1938 & 9.16 & 5,650 & 76.4 & Aug. 19 & b 16.36 & $16,200 \mathrm{~A}$ & 219 \\
\hline 116 & $\begin{array}{l}\text { Hockanum River near East Hartford, } \\
\text { Conn. }\end{array}$ & 74.5 & $\left|\begin{array}{l}1914-21 \\
1928-55\end{array}\right|$ & $\mid$ Sept. 21, 1938| & b 13.78 & 5,160 & 69.3 & Aug. $19,12: 30$ p.m. & 10.46 & 2,740 & - \\
\hline
\end{tabular}




\begin{tabular}{|c|c|}
\hline 117 & Salmon River near East Hampton, Conn. \\
\hline 118 & $\begin{array}{l}\text { East Branch Eightmile River near North } \\
\text { Lyme, Conn. }\end{array}$ \\
\hline 119 & $\begin{array}{l}\text { West Branch Eightmile River at North } \\
\text { Plain, Conn. }\end{array}$ \\
\hline & MENUNKETESUCK RIVER BASIN \\
\hline 120 & $\begin{array}{l}\text { Menunketesuck River near Clinton, Conn, } \\
\text { QUINNIPIAC RIVER BASIN }\end{array}$ \\
\hline 121 & $\begin{array}{l}\text { Quinnipiac River at Wallingford, Conn, } \\
\text { HOUSATONIC RIVER BASIN }\end{array}$ \\
\hline 122 & $\begin{array}{l}\text { Housatonic River near Great Barrington, } \\
\text { Mass. }\end{array}$ \\
\hline 123 & Blackberry River at Canaan, Conn, \\
\hline 124 & $\begin{array}{l}\text { Housatonic River at Falls Village, } \\
\text { Conn. }\end{array}$ \\
\hline 125 & Salmon Creek near Lime Rock, Conn. \\
\hline 126 & Birdseye Brook near Cornwall, Conn. \\
\hline 127 & $\begin{array}{l}\text { Furnace Brook at Cornwall Bridge, } \\
\text { Conn. }\end{array}$ \\
\hline 128 & Tenmile River near Gaylordsville, Conn. \\
\hline $129 *$ & $\begin{array}{l}\text { Housatonic River at Gaylordsville, } \\
\text { Conn. }\end{array}$ \\
\hline 130 & $\begin{array}{l}\text { West Aspetuck River near Merryall, } \\
\text { Conn. }\end{array}$ \\
\hline 131 & Still River at Danbury, Conn, \\
\hline 132 & Still River near Lanesville, Conn. \\
\hline 133 & Bantam River at Litchfield, Conn. \\
\hline & Shepaug River near Roxbury, Conn. \\
\hline
\end{tabular}

\begin{tabular}{|c|c|c|c|}
\hline 105 & $1928-55$ & Sept.21, 1938 & 10.96 \\
\hline 22.0 & $1937-55$ & Sept. 21, 1938 & 7.00 \\
\hline 18.6 & $\mid 1937-55$ & Sept. 21, 1938 & - \\
\hline 11.6 & $1941-55$ & Sept.11, 1954 & 8.51 \\
\hline 109 & $1930-55$ & Sept. 21, 1938 & 9.55 \\
\hline 280 & $1913-55$ & Jan. 1,1949 & 12.08 \\
\hline 48.2 & $1949-55$ & $\begin{array}{l}\text { Nov. } 26,1950 \\
\text { Dec. } 31,1948\end{array}$ & $\begin{array}{l}9.37 \\
12.0\end{array}$ \\
\hline 632 & $1912-55$ & Jan. 1,1949 & b22.9. \\
\hline 30.1 & -- & --- & - \\
\hline 3.88 & - & $--\cdot$ & - \\
\hline 13.5 & --- & $-\cdot$ & - \\
\hline 204 & $1929-55$ & Sept. 22, 1938 & 12.77 \\
\hline 994 & $\begin{array}{l}1900-14 \\
1940-55\end{array}$ & $\begin{array}{l}\text { Jan. } 1,1949 \\
\text { Sept. 22, } 1938\end{array}$ & $\begin{array}{l}14.85 \\
b 14.5\end{array}$ \\
\hline 16.6 & -- & --- & - \\
\hline 38.3 & -- & --- & - \\
\hline 68.5 & $1931-55$ & Sept.22, 1938 & 10.88 \\
\hline 20.6 & $\cdots$ & $-\cdot$ & - \\
\hline 133 & 30 & ot. 21 & b12.8 \\
\hline
\end{tabular}

\begin{tabular}{|c|c|}
\hline 12,400 & 118 \\
\hline 2,950 & 134 \\
\hline 1,810 & 97.3 \\
\hline 1,600 & 138 \\
\hline 5,230 & 48.0 \\
\hline 12,200 & 43.6 \\
\hline 2,550 & 52.9 \\
\hline - & - \\
\hline 23,900 & 37.8 \\
\hline - & - \\
\hline - & - \\
\hline - & - \\
\hline 12,500 & 61.3 \\
\hline $\begin{array}{l}32,300 \\
37,000\end{array}$ & $\begin{array}{l}32.5 \\
37.2\end{array}$ \\
\hline - & - \\
\hline - & - \\
\hline 4,410 & 64.4 \\
\hline - & - \\
\hline
\end{tabular}
See footnotes at end of table p. 29. 


\begin{tabular}{|c|c|c|c|c|c|c|c|c|c|c|c|}
\hline \multirow{2}{*}{$\begin{array}{l}\text { No. } \\
\text { on } \\
\text { pl. } \\
1\end{array}$} & \multirow[b]{2}{*}{ Stream and place of determination } & \multirow{2}{*}{$\begin{array}{l}\text { Drainage } \\
\text { area } \\
(\mathrm{sq} \mathrm{mi})\end{array}$} & \multirow{2}{*}{$\begin{array}{l}\text { Period } \\
\text { of } \\
\text { record }\end{array}$} & \multicolumn{4}{|c|}{ Maximum flood previously known } & \multicolumn{4}{|c|}{ Maximum during present flood } \\
\hline & & & & Date & $\begin{array}{l}\text { Gage } \\
\text { height } \\
\text { (feet) }\end{array}$ & $\begin{array}{l}\text { Discharge } \\
\text { (cfs) }\end{array}$ & $\begin{array}{l}\text { Cfs per } \\
\text { sq mi }\end{array}$ & Date and hour & $\begin{array}{l}\text { Gage } \\
\text { height } \\
\text { (feet) }\end{array}$ & $\begin{array}{l}\text { Discharge } \\
\text { (cfs) }\end{array}$ & $\begin{array}{l}\text { Cfs per } \\
\text { sq mi }\end{array}$ \\
\hline 135 & $\begin{array}{l}\text { HOUSATONIC RIVER BASIN--Continued } \\
\text { Sprain Brook near Hotchkissville, } \\
\text { Conn. }\end{array}$ & 6.91 & -- & -- & - & - & - & Abøut Aug. 19 & - & $2,980 \mathrm{~A}$ & 431 \\
\hline 136 & Nonewaug River near Woodbury, Conn. & 25.6 & $-\cdot-$ & --- & - & - & - & About Aug. 19 & - & $12,300 \mathrm{CE}$ & 480 \\
\hline $137 *$ & Pomperaug River at Southbury, Conn. & 75.3 & $1932-55$ & Sept. 21, 1938 & 16.0 & 7,420 & 98.5 & Aug. $19,6: 30$ a. $\mathrm{m}$. & 21.8 & $29,400 \mathrm{~A}$ & 390 \\
\hline 138 & $\begin{array}{l}\text { Transylvania Brook near Southbury, } \\
\text { Conn. }\end{array}$ & 2.45 & -- & --- & - & $-\cdot$ & - & About Aug. 19 & - & $839 \mathrm{~A}$ & 342 \\
\hline 139 & Housatonic River at Stevenson, Conn. & 1,545 & $1928-55$ & Mar. 12, 1936 & b23.5 & 69,500 & 45.0 & Aug. 19,11 p. m. & 23.42 & 69,300 & 44.9 \\
\hline 140 & $\begin{array}{l}\text { West Branch Naugatuck River near } \\
\text { West Torrington, Conn. }\end{array}$ & 24.2 & -- & $\cdots$ & - & - & - & About Aug. 19 & - & $11,900 \mathrm{D}$ & 492 \\
\hline 141 & $\begin{array}{l}\text { East Branch Naugatuck River near } \\
\text { Torrington, Conn. }\end{array}$ & 10.2 & --- & --- & - & - & - & About Aug. 19 & - & $6,210 \mathrm{~A}$ & 609 \\
\hline $142 *$ & $\begin{array}{l}\text { Naugatuck River near Thomaston, } \\
\text { Conn. }\end{array}$ & 71.9 & $1930-55$ & Dec. 31,1948 & 12.03 & 10,200 & 142 & Aug. $19,5: 30$ a. m. & 24.0 & $41,600 \mathrm{~A}$ & 579 \\
\hline 143 & $\begin{array}{l}\text { Leadmine Brook near Thomaston, } \\
\text { Conn. }\end{array}$ & 24.0 & $1930-55$ & Sept. 17, 1934 & b11.2 & 3,080 & 128 & Aug. 19,5 a. $\mathrm{m}$. & b15. 2 & $10,400 \mathrm{~B}$ & 433 \\
\hline 144 & Branch Brook near Thomaston, Conn. & 21.0 & -- & --- & - & - & - & About Aug. 19 & - & $10,300 \mathrm{C}$ & 490 \\
\hline 145 & Naugatuck River near Waterbury, Conn. & 138 & -- & -- & - & - & - & About Aug. 19 & - & 75,900 & 550 \\
\hline 146 & Hancock Brook near Waterbury, Conn. & 12.9 & -- & -- & - & - & - & About Aug. 19 & - & $4,870 \mathrm{~A}$ & 378 \\
\hline 147 & Steel Brook at Oakville, Conn. & 12.9 & --- & --- & - & - & - & About Aug. 19 & - & $5,890 \mathrm{C}$ & 457 \\
\hline 148 & Mad River at Waterbury, Conn. & 18.0 & --- & --- & - & - & - & About Aug. 19 & - & $2,070 \mathrm{D}$ & 115 \\
\hline 149 & Hop Brook near Naugatuck, Conn. & 16.5 & -- & -- & - & - & - & About Aug. 19 & - & $2,650 \mathrm{C}$ & 161 \\
\hline
\end{tabular}


Naugatuck River near Naugatuck, Conn. SAUGATUCK RIVER BASIN Saugatuck River near Westport, Conn. STREAMS ON LONG ISLAND Cedar Swamp Creek at Glen Cove, N. Y. r Mill Neck Creek at Mill Neck, N. Y. Carlls River at Babylon, N. Y. Massapequa Creek at Massapequa, N.Y. East Meadow Brook at Freeport, N.Y.

Pines Brook at Malverne, N. Y.

\section{HUDSON RIVER BASIN}

Batavia Kill at Hensonville, N. Y.

Nauvo Stream at Windham, N. Y.

Batavia Kill near Ashland, N. Y.

Schoharie Creek at Prattsville, N.Y.

Durham Kill near Oak Hill, N. Y.

Catskill Creek at Oak Hill, N. Y.

Bashbish Creek at Copake Falls, N. Y.

Esopus Creek at Coldbrook, N. Y.

Rondout Creek near Lowes Corners, N, Y.

Chestnut Creek at Grahamsville, N.Y.

Rondout Creek near Lackawack, N. Y. Sandburg Creek at Spring Glen, N.Y.

West Branch Beer Kill near Ellenville,

See footnotes at end of table, p. 29.

\begin{tabular}{|c|c|c|c|c|c|c|}
\hline 246 & $\begin{array}{l}1918-24 \\
1928-55\end{array}$ & Dec. $31,1948 \mid$ & 12.40 & 28,500 & 116 & Aug. 19 \\
\hline 77.5 & $1932-55$ & Mar. 12, 1936 & 11.30 & 5,310 & 68.5 & Aug. $19,10: 30$ a. $\mathrm{m}$. \\
\hline r11 & $1938-55$ & Feb. 7, 1941 & 4.99 & 782 & - & Aug. $12,1: 15$ a. $\mathrm{m}$. \\
\hline r11. 5 & $1937-55$ & Sept. 15, 1944 & - & 104 & - & Aug. 12,3 a. $\mathrm{m}$. \\
\hline r35 & $1944-55$ & Sept. 11, 1954 & 1.495 & 106 & - & Aug. 12,11 p. m. \\
\hline r38 & $1936-55$ & Jan. 7,1944 & 1.616 & 151 & - & Aug. $12,7: 30$ p.m. \\
\hline r31 & $1937-55$ & Feb. 15, 1944 & 2.192 & 297 & - & Aug. $12,9: 45$ a. m. \\
\hline$r 10$ & $1937-55$ & Sept. 11,1954 & $\mathrm{r} 2.56$ & r133 & - & Aug. 12,7 a. m. \\
\hline 13.5 & --- & -- & - & - & - & Aug. 13 \\
\hline 3.88 & --- & --- & - & - & - & Aug. 13 \\
\hline 51.2 &.-- & Dec. 11,1952 & - & 7,090 & 138 & Aug. 13 \\
\hline 236 & $1907-55$ & $\begin{array}{l}\text { Sept. 21, 1938 } \\
\text { Nov. 25, 1950 }\end{array}$ & $\begin{array}{l}\text { bi15.6 } \\
\text { i15.50 }\end{array}$ & 49,500 & $210^{-}$ & $\begin{array}{l}\text { Aug. } 13,4: 45 \text { p. m. } \\
\text { Aug. } 18,3 \text { p. m. }\end{array}$ \\
\hline 5.82 & -- & -- & - & - & - & Aug. 13 \\
\hline 98 & $1929-55$ & Nov. 25,1950 & 14.08 & 12,500 & 128 & Aug. $18,1: 15$ p. m. \\
\hline 15.8 & -. & --- & - & - & - & Aug. 19 \\
\hline 192 & $1914-55$ & Mar. 30, 1951 & 20.70 & 59,600 & 310 & Aug. $19,2: 30$ a. $\mathrm{m}$. \\
\hline 38.5 & $1937-55$ & $\begin{array}{l}\text { July } 22,1938 \\
\text { Mar. 30, 1951 }\end{array}$ & $\begin{array}{l}\text { i } 8.2 \\
\text { b, i } 9.60\end{array}$ & 7,600 & 197 & Aug. $18,11: 45$ p.m. \\
\hline 20.9 & $1938-55$ & Nov. 25,1950 & t 4.27 & 3,800 & 182 & Aug. 18,11 p. m. \\
\hline 100 & $1906-55$ & Aug. 26, 1928 & - & 26,700 & 267 & Aug. $19,1: 15$ a.m. \\
\hline 27.3 & --- & June 1,1952 & - & 1,850 & 67.8 & Aug. 19 \\
\hline 19.7 & -- & --- & - & - & - & Aug. 19 \\
\hline
\end{tabular}

431

32.6 


\begin{tabular}{|c|c|c|c|c|c|c|c|c|c|c|c|}
\hline \multirow{2}{*}{$\begin{array}{c}\text { No. } \\
\text { on } \\
\text { pl. } \\
1\end{array}$} & \multirow[b]{2}{*}{ Stream and place of determination } & \multirow{2}{*}{$\begin{array}{c}\text { Drainage } \\
\text { area } \\
\text { (sq } \mathrm{mi})\end{array}$} & \multirow{2}{*}{$\begin{array}{l}\text { Period } \\
\text { of } \\
\text { record }\end{array}$} & \multicolumn{4}{|c|}{ Maximum flood previously known } & \multicolumn{4}{|c|}{ Maximum during present flood } \\
\hline & & & & Date & $\begin{array}{c}\text { Gage } \\
\text { height } \\
\text { (feet) }\end{array}$ & $\begin{array}{c}\text { Discharge } \\
\text { (cfs) }\end{array}$ & $\begin{array}{l}\text { Cfs per } \\
\text { sq mi }\end{array}$ & Date and hour & $\begin{array}{c}\text { Gage } \\
\text { height } \\
\text { (feet) }\end{array}$ & $\begin{array}{c}\text { Discharge } \\
\text { (cfs) }\end{array}$ & $\begin{array}{l}\text { Cfs per } \\
\text { sq mi }\end{array}$ \\
\hline 172 & $\begin{array}{l}\text { HUDSON RIVER BASIN--Continued } \\
\text { Fantine Kill at Ellenville, N. Y. }\end{array}$ & 2.46 & --- & -- & - & - & - & Aug. 19 & - & $1,410 \mathrm{~A}$ & 573 \\
\hline 173 & Stony Kill near Granite, N. Y. & 6.32 & $\cdots$ & -- & - & - & - & Aug. 19 & - & 3,520 & 557 \\
\hline 174 & Peters Kill near Accord, N. Y. & 5.53 & -- & -- & - & - & - & Aug. 19 & - & 2,550 & 461 \\
\hline $175 *$ & Rondout Creek at Rosendale, N. Y. & 386 & $1926-55$ & Aug. 27, 1928 & 21.9 & 27,300 & 70.7 & Aug. 19 , about 6 a.m. & 23.93 & u30, 900B & u106 \\
\hline 176 & $\begin{array}{l}\text { Clove River at Clove Acres outlet, at } \\
\text { Sussex, N. J. }\end{array}$ & 19.7 & -- & $-\cdots$ & - & - & - & Aug. 19 & - & 5,780 & 293 \\
\hline 177 & Wallkill River near Unionville, N.Y. & 144 & $1937-55$ & Dec. 31,1948 & 11.4 & 5,000 & 34.7 & Aug. 19,9 p. m. & 13.35 & 6,880 & 47.8 \\
\hline 178 & Rutgers Creek at Gardnerville, N.Y. & 59.7 & $1943-55$ & Dec. 31,1948 & 8.88 & 3,600 & 60.3 & Aug. 19 , about 9 a.m. & 12.38 & $8,490 \mathrm{~A}$ & 142 \\
\hline 179 & Pochuck Creek near Pine Island, N.Y. & 98.0 & $1937-55$ & Sept. 22, 1938 & 7.80 & 2,070 & 21.1 & Aug. 20,7 a. $\mathrm{m}$. & 8.43 & $2,870 \mathrm{~B}$ & 29.3 \\
\hline 180 & Quaker Creek at Florida, N. Y. & 9.74 & $1937-55$ & Sept. 21, 1938 & 6.0 & 1,050 & 108 & Aug. $19,4: 15$ a. $\mathrm{m}$. & 5.27 & 619 & 63.6 \\
\hline 181 & $\begin{array}{l}\text { Wallkill River at Pellets Island } \\
\text { Mountain, N. Y. }\end{array}$ & 385 & $1920-55$ & Mar. 14, 1936 & 25.0 & 12,400 & 32.2 & Aug. $21,9: 30$ a. $\mathrm{m}$. & 21.76 & 10,100 & 26.2 \\
\hline 182 & Wallkill River near Philipsburg, N. Y. & 419 & $1936-55$ & $\begin{array}{l}\text { Dec. } 31,1948 \\
\text { Mar. } 13,1936\end{array}$ & $\begin{array}{l}9.94 \\
11.3\end{array}$ & 7,380 & $\begin{array}{c}17.6 \\
-\end{array}$ & Aug. 21,1 p.m. & 11.33 & 9,200 & 22.0 \\
\hline 183 & Shawangunk Kill at Ganahgote, N. Y. & 147 & -- & June 1,1952 & - & 10,500 & 71.4 & Aug. 19 & - & 15,700 & 107 \\
\hline $184 *$ & Wallkill River at Gardiner, N. Y. & 711 & $1924-55$ & $\begin{array}{ll}\text { Mar. } 7,1945 \\
\text { June } 1,1952\end{array}$ & $\begin{array}{r}k 18.83 \\
14.88\end{array}$ & 21,200 & 29.8 & Aug. $19,10: 30$ a. $\mathrm{m}$. & 19.77 & 30,600 & 43.0 \\
\hline $185 *$ & $\begin{array}{l}\text { Wappinger Creek near Wappingers } \\
\text { Falls, N. Y. }\end{array}$ & 182 & $1928-55$ & Sept. 22, 1938 & 18.02 & 15,900 & 87.4 & Aug. 19 , about 3 p. $m$ & 19.60 & $18,600 \mathrm{CE}$ & 102 \\
\hline 186 & Fishkill Creek at Beacon, N. Y. & 186 & $1944-55$ & Jan. 25, 1953 & 7.28 & 3,220 & 17.3 & Aug. 20,4 a. $\mathrm{m}$. & 12.13 & 8,800 & 47.3 \\
\hline 187 & Woodbury Creek near Mountainville,N. Y & 17.5 & -- & -- & - & - & - & Aug. 19 & - & $1,520 \mathrm{~A}$ & 86.9 \\
\hline
\end{tabular}


188

Moodna Creek at Firthcliffe, N. Y. HACKENSACK RIVER BASIN

189

190

191

Hackensack River at Rivervale, N. J.

Pascack Brook at Westwood, N. J.

Hackensack River at New Milford, N.J.

PASSAIC RIVER BASIN

Passaic River near Millington, N. J.

Passaic River near Chatham, N. J.

Rockaway River above reservoir

at Boonton, N. J.

Whippany River at Morristown, N.J.

Greenwood Lake at Awosting, N. J.

Wanaque River at Awosting, N. J.

Wanaque River at Monks, N. J.

Ringwood Creek near Wanaque, N.J. Cupsaw Brook near Wanaque, N. J.

Blue Mine Brook near Wanaque, N. J.

Ramapo River near Mahwah, N. J.

Ramapo River at Pompton Lakes, N. J.

Saddle River at Ridgewood, N.J.

See footnotes at end of table, p. 29

\begin{tabular}{|c|c|c|c|c|c|}
\hline 162 & $-\cdot$ & -- & - & - & - \\
\hline 58.0 & $1941-55$ & Apr. 1,1951 & 5.70 & 1,350 & 23.3 \\
\hline 29.6 & $1934-55$ & July 23, 1945 & 6.76 & 1,610 & 54.4 \\
\hline 113 & $1921-55$ & Mar. 31, 1951 & 6.14 & 3,660 & 32.4 \\
\hline 55.4 & $\begin{array}{l}1903-6, \\
1921-55\end{array}$ & Mar. 8, 1904 & i 7.50 & 2,000 & 36.1 \\
\hline 100 & \begin{tabular}{|}
$\mid 1903-11$ \\
$1937-55$
\end{tabular} & Jan. 9,1905 & i 8.3 & r 3,000 & 30.0 \\
\hline 116 & $1937-55$ & June 2,1952 & 6.62 & 3,250 & 28.0 \\
\hline 29.4 & $1921-55$ & $\begin{array}{l}\text { Aug. 26, } 1928 \\
\text { Mar. 12, } 1936 \\
\text { Mar. 15, 1940 }\end{array}$ & $\begin{array}{l}7.30 \\
- \\
-\end{array}$ & $\begin{array}{l}1,500 \\
1,500 \\
1,500\end{array}$ & $\begin{array}{l}51.0 \\
51.0 \\
51.0\end{array}$ \\
\hline 27.1 & $\begin{array}{l}1898- \\
1903, \\
1907-55\end{array}$ & Oct.9-14, 1903 & r14.25 & - & - \\
\hline 27.1 & $1919-55$ & Mar. 31, 1951 & 5.44 & 1,190 & 43.9 \\
\hline 40.4 & $1935-55$ & Mar. 31, 1951 & b 3.85 & 2,660 & 65.8 \\
\hline 19.1 & $1934-55$ & Mar. 31, 1951 & b 3.74 & 1,150 & 60.2 \\
\hline 4. 38 & $1934-55$ & Mar. 11, 1936 & 2.94 & 536 & 122 \\
\hline 1.71 & $1934-55$ & Mar. 30, 1951 & 2.15 & 458 & 268 \\
\hline 118 & $\begin{array}{l}1903-6 \\
1922-55\end{array}$ & Oct. 9,1903 & i 9.8 & $\mathrm{r} 12,400$ & 105 \\
\hline 160 & $1921-55$ & Mar. 12, 1936 & 3.56 & 12,300 & 76.9 \\
\hline r21.4 & $1954-55$ & -- & - & - & - \\
\hline
\end{tabular}

Aug. 19

Aug. 19,7 p. m

Aug. 19,7 p. $\mathrm{m}$

Aug. 19,1 p. $m$

Aug. 19, 2-4 p. m.

Aug. 14,2 p. $m$

Aug. 19,5 a. $m$.

b 6.31

Aug. $19,7: 30$ p.m.

Aug. 19, 9:30 a.m.

Aug. 19

Aug. 19

Aug. 19

Aug. 19, 8:30 a.m

Aug. 19, 6:30 a.m.

Aug. 19, 5 a. m.

Aug. $19,1: 30$ p. m.

Aug. 19, 6-7 p.m.

Aug. 13,2 p. $\mathrm{m}$. Aug. 19,10 a.m.
5.43

5.61

1,16

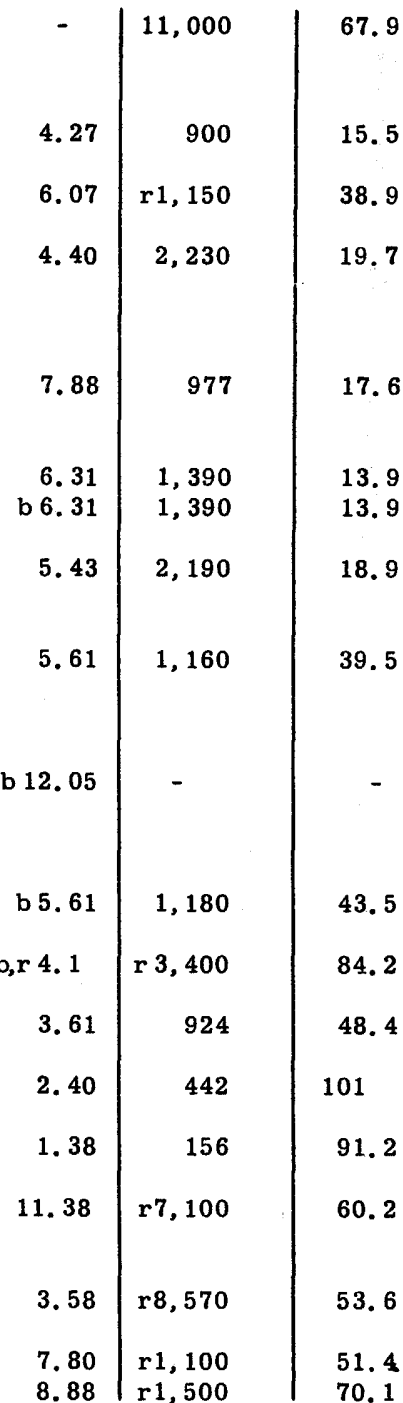




\begin{tabular}{|c|c|c|c|c|c|c|c|c|c|c|c|}
\hline \multirow[b]{2}{*}{$\begin{array}{c}\text { No. } \\
\text { on } \\
\text { pl. } \\
1\end{array}$} & \multirow[b]{2}{*}{ Stream and place of determination } & \multirow[b]{2}{*}{$\begin{array}{l}\text { Drainage } \\
\text { area } \\
\text { (sq } \mathrm{mi} \text { ) }\end{array}$} & \multirow[b]{2}{*}{$\begin{array}{l}\text { Period } \\
\text { of } \\
\text { record }\end{array}$} & \multicolumn{4}{|c|}{ Maximum flood previously known } & \multicolumn{4}{|c|}{ Maximum during present flood } \\
\hline & & & & Date & $\begin{array}{l}\text { Gage } \\
\text { height } \\
\text { (feet) }\end{array}$ & $\begin{array}{c}\text { Discharge } \\
\text { (cfs) }\end{array}$ & $\begin{array}{l}\text { Cfs per } \\
\text { sq mi }\end{array}$ & Date and hour & $\begin{array}{l}\text { Gage } \\
\text { height } \\
\text { (feet) }\end{array}$ & $\begin{array}{l}\text { Discharge } \\
\text { (cfs) }\end{array}$ & $\begin{array}{l}\text { Cfs per } \\
\text { sq mi }\end{array}$ \\
\hline 205 & $\begin{array}{l}\text { PASSAIC RIVER BASIN--Continued } \\
\text { Hohokus Brook at Hohokus, N. J. }\end{array}$ & $\mathbf{r} 16.5$ & $1954-55$ & -- & - & - & - & $\begin{array}{l}\text { Aug. } 13,10 \text { a. m. } \\
\text { Aug. } 19,6: 30 \text { a. m. }\end{array}$ & $\begin{array}{l}3.49 \\
4.71\end{array}$ & $\begin{array}{r}\mathbf{r} 700 \\
\mathrm{r} 1,400\end{array}$ & $\begin{array}{l}42.4 \\
84.8\end{array}$ \\
\hline 206 & $\begin{array}{l}\text { Saddle River at Lodi, N. J. } \\
\text { RARITAN RIVER BASIN }\end{array}$ & 54.6 & $1923-55$ & July 23,1945 & $\mathrm{~b} 10.00$ & 3,500 & 64.1 & Aug. 19,10 p. m. & 6.47 & 2,200 & 40.3 \\
\hline 207 & $\begin{array}{l}\text { South Branch Raritan River near } \\
\text { High Bridge, N. J. }\end{array}$ & 65.3 & $1919-55$ & Mar. 15, 1940 & 11.78 & 5,160 & 69.0 & Aug. 19,9 a. m. & 10.48 & 2,780 & 42.6 \\
\hline 208 & $\begin{array}{l}\text { South Branch Raritan River at } \\
\text { Clinton, N. J. }\end{array}$ & 112 & --- & $-\therefore$ & - & - & - & Aug. 19 & - & $8,080 \mathrm{D}$ & 72.1 \\
\hline 209 & Cakepaulin Creek at Lansdowne, N. J. & 13.7 & --- & --- & - & - & - & Aug. 18 & - & 7,230 & 528 \\
\hline $210 *$ & $\begin{array}{l}\text { South Branch Raritan River at } \\
\text { Stanton, N. J. }\end{array}$ & 147 & $\begin{array}{l}1903-6 \\
1919-55\end{array}$ & $\mid \begin{array}{l}\text { Niar. 15, 1940, } \\
\text { July } 19,1945\end{array}$ & 12.2 & 10,900 & 74.1 & Aug. 19,2 a. m. & 15.22 & $18,000 \mathrm{AB}$ & 122 \\
\hline 211 & Prescott Brook near Stanton, N. J. & 8.37 & 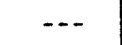 & $\cdots$ & - & - & - & Aug. 18 & - & 2.010 & 240 \\
\hline 212 & Walnut Brook near Flemington, N.J. & 2.24 & $1936-55$ & July 18,1945 & 3.30 & 645 & 288 & Aug. 18,12 p. m. & 3.02 & 443 & 198 \\
\hline 213 & Neshanic River at Reaville, N. J. & 25.7 & $1930-55$ & July 18,1945 & b12.33 & 5,940 & 231 & Aug. 19,1 a. $\mathrm{m}$ & b11.90 & 8,830 & 344 \\
\hline 214 & $\begin{array}{l}\text { North Branch Raritan River near } \\
\text { Far Hills, N. J. }\end{array}$ & 26.2 & $1922-55$ & $\mid$ Mar. 15, 1940 & 5.85 & 3,410 & 130 & Aug. $19,3: 30$ p. m. & 4.95 & r 1,700 & 64.9 \\
\hline 215 & $\begin{array}{l}\text { Lamington (Black) River near } \\
\text { Pottersville, N. J. }\end{array}$ & 32.8 & $1921-55$ & Mar. 15, 1940y & v5. 34 & $\mathrm{v} 2,630$ & 80.2 & $\begin{array}{l}\text { Aug. } 13,4: 30 \text { p. m. } \\
\text { Aug. } 18,10 \text { p. m. }\end{array}$ & $\begin{array}{l}5.24 \\
4.71\end{array}$ & $\begin{array}{l}2,480 \\
1,770\end{array}$ & $\begin{array}{l}75.6 \\
54.0\end{array}$ \\
\hline 216 & $\begin{array}{l}\text { North Branch Raritan River near } \\
\text { Raritan, N. J. }\end{array}$ & 190 & $1923-55$ & Sept. 21, 1938 & 12.16 & 16,500 & 86.8 & $\begin{array}{l}\text { Aug. } 13,12: 30 \text { p. } \mathrm{m} . \\
\text { Aug. } 19,7 \text { a. m. }\end{array}$ & $\mid \begin{array}{ll}b & 12.70 \\
\text { b } & 13.59\end{array}$ & 20,700 & $109^{-}$ \\
\hline 217 & Raritan River at Manville, N. J. & 490 & $\begin{array}{l}1903-7 \\
1921-55\end{array}$ & Sept. 22, 1938 & $\mathrm{~b} 20.42$ & - & - & Aug. 19,4 p. m. & b 22.06 & 33,300 & 68.0 \\
\hline 218 & Stony Brook at Princeton, N. J. & $\mathrm{r} 44.0$ & $1953-55$ & WDec. 14,1953 & b8. 66 & - & - & Aug. 13, 10:30 a.m. & 11.90 & $\mathrm{r} 4,600$ & 105 \\
\hline
\end{tabular}

218 Stony Brook at Princeton, N. J. 


\begin{tabular}{|c|c|c|c|c|c|c|c|c|c|c|c|}
\hline 219 & Millstone River at Lake Carnegie, N.J. & 159 & $1926-55$ & ||Sept. 21, 1938 & b4.20 & - & - & $\begin{array}{l}\text { Aug. } 13,4 \text { p. m. } \\
\text { Aug. } 19,10 \text { a.m. }\end{array}$ & $\begin{array}{r}\text { b5.02 } \\
4.53\end{array}$ & - & $\begin{array}{l}- \\
-\end{array}$ \\
\hline 220 & Millstone River at Blackwells Mills, N. Jf & 258 & $1921-55$ & |Sept. 21, 1938 & b15.29 & - & - & $\begin{array}{l}\text { Aug. } 14,1 \text { a. } \mathrm{m} \\
\text { Aug. } 19,6 \text { p. } \mathrm{m} .\end{array}$ & $\begin{array}{l}12.42 \\
12.14\end{array}$ & - & - \\
\hline 221 & Raritan River at Bound Brook, N.J. & 779 & $\begin{array}{l}1903-9 \\
1945-55\end{array}$ & Sept. 22, 1938 & b16. 3 & - & - & Aug. 19,4 p. m. & 16.20 & 30,800 & 39.5 \\
\hline 222 & Green Brook at Plainfield, N. J. & 9.75 & $1938-55$ & July 23,1938 & 5.82 & 2,890 & 296 & $\begin{array}{l}\text { Aug. } 13,8 \text { a. } \mathrm{m} \text {. } \\
\text { Aug. } 19,3 \text { a. } \mathrm{m} \text {. }\end{array}$ & $\begin{array}{l}4.32 \\
3.30\end{array}$ & $\begin{array}{r}1,270 \\
644\end{array}$ & $\begin{array}{r}130 \\
66.1\end{array}$ \\
\hline 223 & Lawrence Brook at Farrington Dam, N. J & 34.4 & $1927-55$ & Sept. 21, 1938 & 26.18 & 2,660 & 77.3 & Aug. $13,10: 30$ a. $\mathrm{m}$. & 25.78 & 1,450 & 42.2 \\
\hline 224 & $\begin{array}{r}\text { South River at Old Bridge, N. J. } \\
\text { DELAWARE RIVER BASIN }\end{array}$ & 94.6 & $1939-55$ & Sept. 15, 1944 & 11.71 & 4,250 & 44.9 & Aug. 13,7 p. m. & 11.27 & 2,680 & 28.3 \\
\hline 225 & Callicoon Creek at Callicoon, N. Y. & 111 & $1940-55$ & Aug. 17, 1947 & 9.68 & 16,000 & 144 & Aug. 19,2 a. m. & 7.11 & 8,150 & 73.4 \\
\hline 226 & Tenmile River at Tusten, N. Y. & 45.0 & $1946-55$ & Nov. 26,1950 & 6.05 & 1,870 & 41.6 & Aug. 19,3 a. $\mathrm{m}$. & 9.08 & 6,850 & 150 \\
\hline $227 *$ & $\begin{array}{l}\text { Dela ware River above Lackawaxen } \\
\text { River, near Barryville, N. Y. }\end{array}$ & 2,023 & $1940-55$ & May 23,1942 & 23.19 & 105,000 & 51.9 & $\begin{array}{l}\text { Aug. } 19, \\
\text { about } 3: 30 \text { a. m. }\end{array}$ & 26.40 & $w 130,000$ & w78. 7 \\
\hline 228 & $\begin{array}{l}\text { West Branch Lackawaxen River at } \\
\text { Prompton, } \mathrm{Pa} \text {. }\end{array}$ & 59.7 & $1944-55$ & $\mid \begin{array}{l}\text { Apr. } 5,1947 \\
\text { Nov. 25, 1950 } \\
\text { May 23, 1942 }\end{array}$ & $\begin{array}{r}8.45 \\
8.42 \\
\mathrm{~b} 16.7\end{array}$ & $\begin{array}{l}5,230 \\
5,230 \\
-\end{array}$ & $\begin{array}{l}87.6 \\
87.6 \\
-\end{array}$ & Aug. 18,11 p. m. & 9.24 & 5,860 & 98.2 \\
\hline 229 & Dyberry Creek at Dyberry, $\mathrm{Pa}$. & 63.2 & $1943-55$ & $\mid \begin{array}{ll}\text { July } 10,1952 \\
\text { May } 23,1942\end{array}$ & $\begin{array}{c}14.6 \\
b 15.86\end{array}$ & $v 15,500$ & 245 & Aug. $18,11: 30$ p.m. & 13.78 & $11,400 \mathrm{~A}$ & 180 \\
\hline 230 & Lackawaxen River near Honesdale, $\mathrm{Pa}$. & 164 & $1948-55$ & $\mid$\begin{tabular}{|rr} 
July & 10,1952 \\
May & 1942
\end{tabular} & $\begin{array}{l}14.2 \\
24.5\end{array}$ & $\begin{array}{r}12,400 \\
\mathrm{r} 34,000\end{array}$ & $\begin{array}{l}75.6 \\
207\end{array}$ & Aug. $18,11: 30$ p.m. & 15.52 & 18,600 & 113 \\
\hline $231 *$ & Middle Creek near Hawley, $\mathrm{Pa}$. & 78.4 & $1944-55$ & 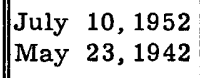 & $\mathrm{r}^{14.17}$ & $\begin{array}{l}6,970 \\
-\end{array}$ & $\begin{array}{c}88.9 \\
-\end{array}$ & Aug. 19,1 a. m. & 17.87 & $12,000 \mathrm{~A}$ & 153 \\
\hline $232 *$ & Lackawaxen River at Hawley, $\mathrm{Pa}$. & 518 & $\begin{array}{l}1908-19 \\
1938-55\end{array}$ & May 23, 1942 & 20.1 & 50,000 & a 172 & Aug. 19,2 . a. m. & 20.6 & 51,900 & a179 \\
\hline 233 & $\begin{array}{l}\text { Wallenpaupack Creek at South } \\
\text { Sterling, } \mathrm{Pa} \text {. }\end{array}$ & 14.3 & -- & May & - & y 10,600 & - & Aug. 19 , about $1 \mathrm{a} . \mathrm{m}$. & - & $22,200 \mathrm{~A}$ & 1,550 \\
\hline 234 & Angels Creek at Angels, $\mathrm{Pa}$. & 1.33 & $-\cdot$ & $\cdots$ & - & - & - & Aug. 18 & - & $1,070 \mathrm{E}$ & 805 \\
\hline 235 & $\begin{array}{l}\text { East Branch Wallenpaupack, Creek at } \\
\text { Greentown, Pa. }\end{array}$ & 33.9 & -- & $\cdots$ & - & - & - & $\begin{array}{l}\text { Aug. } 19 \\
\text { about } 12: 30 \text { a.m. }\end{array}$ & - & $33,300 \mathrm{~A}$ & 982 \\
\hline
\end{tabular}




\begin{tabular}{|c|c|c|c|c|c|c|c|c|c|c|c|}
\hline \multirow[b]{2}{*}{$\begin{array}{c}\text { No. } \\
\text { on } \\
\text { pl. } \\
1\end{array}$} & \multirow[b]{2}{*}{ Stream and place of determination } & \multirow[b]{2}{*}{$\begin{array}{l}\text { Drainage } \\
\text { area } \\
\text { (sq mi) }\end{array}$} & \multirow[b]{2}{*}{$\begin{array}{c}\text { Period } \\
\text { of } \\
\text { record }\end{array}$} & \multicolumn{4}{|c|}{ Maximum flood previously known } & \multicolumn{4}{|c|}{ Maximum during present flood } \\
\hline & & & & Date & $\begin{array}{l}\text { Gage } \\
\text { height } \\
\text { (feet) }\end{array}$ & $\begin{array}{c}\text { Discharge } \\
\text { (cfs) }\end{array}$ & $\begin{array}{l}\text { Cfs per } \\
\text { sq mi }\end{array}$ & Date and hour & $\begin{array}{c}\text { Gage } \\
\text { height } \\
\text { (feet) }\end{array}$ & $\begin{array}{c}\text { Discharge } \\
\text { (cfs) }\end{array}$ & $\begin{array}{l}\text { Cfs per } \\
\text { sq mi }\end{array}$ \\
\hline 236 & $\begin{array}{l}\text { DELAWARE RIVER BASIN--Continued } \\
\text { West Branch Wallenpaupack Creek near } \\
\text { Sterling, } \mathrm{Pa} \text {. }\end{array}$ & 52.4 & $-\cdots$ & May $\quad 1942$ & - & z 7,720 & - & Aug. 19 & - & $14,000 \mathrm{~A}$ & 267 \\
\hline 237 & Butternut Creek near Sterling, $\mathrm{Pa}$. & 5.03 & --- & $\cdots$ & - & - & - & Aug. 18-19 & - & $3,520 \mathrm{~A}$ & 700 \\
\hline 238 & Wallenpaupack Creek at Wilsonville, $\mathrm{Pa}$. & 228 & $\begin{array}{l}1913-22 \\
1925-55\end{array}$ & --- & - & - & - & $\begin{array}{l}\text { Aug. } 19,11 \mathrm{p.m} . \text { to } \\
\text { Aug. } 21,11 \mathrm{p.m} .\end{array}$ & aa3. 45 & $a b 2,900$ & - \\
\hline 239 & Shohola Creek at Lords Valley, $\mathrm{Pa}$. & 25.2 & --- & -- & - & - & - & Aug. 19 & - & $13,800 \mathrm{~A}$ & 548 \\
\hline 240 & Mongaup River near Mongaup, N.Y. & 202 & $1939-55$ & Apr. 9,1940 & - & ac 4,240 & 21.0 & Aug. $19,4: 30$ a. m. & 11.22 & r,ac10, 800 & 53.5 \\
\hline 241 & Shingle Kill near Sparrow Bush, N. Y. & 12.1 & $--\cdot$ & --- & - & - & - & Aug. 19 & - & $6,590 \mathrm{~A}$ & 545 \\
\hline $242 *$ & Delaware River at Port Jervis, N. Y. & 3,076 & $1904-55$ & $\begin{array}{l}\text { May } 23,1942 \\
\text { Oct. } 10,1903\end{array}$ & $\begin{array}{l}17.76 \\
23.1\end{array}$ & $\begin{array}{r}\mathrm{p} 140,000 \\
205,000\end{array}$ & $\begin{array}{l}45.5 \\
66.6\end{array}$ & $\begin{array}{l}\text { Aug. } 19 \\
\text { about } 5: 30 \text { a. m. }\end{array}$ & 23.91 & $\mathrm{p} 233,000 \mathrm{~A}$ & 75.7 \\
\hline $243 *$ & $\begin{array}{l}\text { Neversink River at Oakland Valley, } \\
\text { N. Y. }\end{array}$ & 222 & $1928-55$ & Nov. 26,1950 & 12.62 & 23,300 & 105 & Aug. 19, 2:30 a.m. & 12.74 & $\operatorname{ad23}, 800$ & $\operatorname{ad} 183$ \\
\hline 244 & Gumaer Brook near Wurtsboro, N. Y & 6.92 & $\cdots$ & --- & - & - & - & Aug. 19 & - & $2,870 \mathrm{~A}$ & 415 \\
\hline 245 & Pine Kill near West Brookville, N. Y. & 10.7 & $-\cdots$ & -- & - & - & - & Aug. 19 & - & 2,640 & 247 \\
\hline 246 & Basher Kill at West Brookville, N. Y. & 59.5 & --- & --- & - & - & - & Aug. 19 & - & 4,040 & 67.9 \\
\hline 247 & Neversink River at Godeffroy, N. Y. & 302 & $1937-55$ & Nov. 26, 1950 & 11.79 & 20,000 & 66.2 & Aug. 19,4 a. m. & 12.49 & $\operatorname{ad} 33,000 \mathrm{~A}$ & $\operatorname{ad} 157$ \\
\hline $248 *$ & Delaware River at Montague, N. J. & 3,480 & $1940-55$ & $\begin{array}{l}\text { May } 23,1942 \\
\text { Oct. } 10,1903\end{array}$ & $\begin{array}{l}25.70 \\
35.5\end{array}$ & 136,500 & 39.2 & Aug. 19,9 a. $\mathrm{m}$. & 35.15 & - & - \\
\hline 249 & $\begin{array}{l}\text { Dingmans Creek at Dingmans } \\
\text { Ferry, } \mathrm{Pa} \text {. }\end{array}$ & 15.2 & --- & --- & - & - & - & Aug. 19 & - & $3,660 \mathrm{~B}$ & 241 \\
\hline 250 & Bush Kill near Shoemakers, Pa. & 85.6 & -- & $\cdots$ & - & - & - & Aug. 19 & - & $19,500 \mathrm{~A}$ & 228 \\
\hline 251 & Saw Creek near Shoemakers, Pa. & 29.9 & --- & $\cdots$ & - & - & - & Aug. 19 & - & $3,870 \mathrm{~A}$ & 129 \\
\hline
\end{tabular}


252* Bush Kill at Shoemakers, $\mathrm{Pa}$.

253 Big Flat Brook near Hainesville, N.J.

254* Flat Brook near Flatbrookville, N. J.

255

Middle Branch Brodhead Creek near Canadensis, $\mathrm{Pa}$.

256

257

258

259

260* Brodhead Creek at Minisink Hills, $\mathrm{Pa}$,

261 Paulens Kill at Lafayette, N. J.

262 Culvers Brook at Culvers Pond near Branchville, N. J.

263 Culvers Brook at Branchville, N. J.

264 Dry Brook at Branchville, N. J.

265 Paulins Kill at Paulins Kill Lake Dam near Newton, N. J.

266 Trout Brook at Middleville, N. J.

267* Paulins Kill at Blairstown, N. J.

268 Pequest River at Huntsville, N. J.

269 Pequest River at Pequest, N. J.

270 Beaver Brook near Belvidere, N. J.

271* Delaware River at Belvidere, N. J.

272* Lehigh River at Stoddartsville, $\mathrm{Pa}$.

\begin{tabular}{|c|c|c|c|}
\hline 117 & $1908-55$ & $\mid$ July 24,1920 & 7.2 \\
\hline 19.9 & -- & $-\cdots$ & - \\
\hline 65.1 & $1923-55$ & Apr. 6,1952 & 7.24 \\
\hline 6.14 & -- & -- & - \\
\hline 12.6 & $\ldots$ & -- & - \\
\hline 124 & -- & $\cdots$ & - \\
\hline 65.3 & $1911-38$ & Mar. 12, 1936 & b10.5 \\
\hline 37.7 & $1911-19$ & $\begin{array}{l}\text { Jan. } 7,1915 \\
\text { Feb. 26, } 1918\end{array}$ & $\begin{array}{l}6.52 \\
7.1\end{array}$ \\
\hline 259 & $1950-55$ & Dec. 1, 1952 & 14.43 \\
\hline 33.6 & -- & -- & - \\
\hline 7.12 & -- & --- & - \\
\hline 10.7 & $\ldots$ &.-- & - \\
\hline 4.80 & -- & -- & - \\
\hline 81 & --- & --- & - \\
\hline 5.50 & $\ldots$ & $\ldots$ & - \\
\hline 126 & $1921-55$ & Sept. 22, 1938 & 7.56 \\
\hline 31.4 & $1939-55$ & July 20,1945 & 4.68 \\
\hline 108 & $1921-55$ & Mar. 14, 1936 & 4.97 \\
\hline 36.2 & $1922-55$ & Mar. 12, 1936 & 5.76 \\
\hline 4,535 & $1922-55$ & $\begin{array}{l}\text { Mar. } 19,1936 \\
\text { Oct. } 10,1903\end{array}$ & $\begin{array}{r}25.0 \\
\mathrm{~b} 28.6\end{array}$ \\
\hline 91.7 & $1943-55$ & $\left|\begin{array}{lr}\text { Dec. } & 4,1950 \\
\text { May } & 22,1942\end{array}\right|$ & $\begin{array}{r}8.59 \\
12.03\end{array}$ \\
\hline
\end{tabular}

\begin{tabular}{|c|c|c|}
\hline 13.95 & $23,400 \mathrm{~A}$ & 200 \\
\hline - & 4,140 & 208 \\
\hline b12.58 & $9,550 \mathrm{~A}$ & 147 \\
\hline - & $3,840 \mathrm{BD}$ & 625 \\
\hline - & $8,870 \mathrm{D}$ & 704 \\
\hline - & $72,200 \mathrm{~A}$ & 582 \\
\hline b 14.1 & $5,740 \mathrm{BE}$ & 87.9 \\
\hline - & $22,400 \mathrm{~A}$ & 594 \\
\hline b 29.9 & $68,800 \mathrm{~A}$ & 266 \\
\hline - & 1,440 & 42.9 \\
\hline - & 444 & 62.4 \\
\hline - & 1,560 & 146 \\
\hline - & 1,790 & 373 \\
\hline - & 6,020 & 74.3 \\
\hline - & 899 & 163 \\
\hline 11.12 & $8,740 A$ & 69.4 \\
\hline 5.06 & 565 & 18.0 \\
\hline 3.86 & 1,110 & 10.3 \\
\hline 4.40 & 750 & 20.7 \\
\hline 30.21 & $247,000 \mathrm{~A}$ & 54.5 \\
\hline 16.37 & $31,900 \mathrm{~A}$ & 348 \\
\hline
\end{tabular}

1
8
8
8 
Table 2. --Summary of stages and discharges in the Northeast during the floods of August 1955--Continued

\begin{tabular}{|c|c|c|c|c|c|c|c|c|c|c|c|}
\hline \multirow[b]{2}{*}{$\begin{array}{c}\text { No. } \\
\text { on } \\
\text { pl. } \\
1\end{array}$} & \multirow[b]{2}{*}{ Stream and place of determination } & \multirow[b]{2}{*}{$\begin{array}{l}\text { Drainage } \\
\text { area } \\
\text { (sq mi) }\end{array}$} & \multirow[b]{2}{*}{$\begin{array}{l}\text { Period } \\
\text { of } \\
\text { record }\end{array}$} & \multicolumn{4}{|c|}{ Maximum flood previously known } & \multicolumn{4}{|c|}{ Maximum diring presert flood } \\
\hline & & & & Date & $\begin{array}{c}\text { Gage } \\
\text { height } \\
\text { (feet) }\end{array}$ & $\begin{array}{c}\text { Discharge } \\
\text { (cfs) }\end{array}$ & $\begin{array}{l}\text { Cfs per } \\
\text { sq mi }\end{array}$ & Date and hour & $\begin{array}{l}\text { Gage } \\
\text { height } \\
\text { (feet) }\end{array}$ & $\begin{array}{l}\text { Discharge } \\
\text { (cfs) }\end{array}$ & $\begin{array}{l}\text { Cfs per } \\
\text { sq ni }\end{array}$ \\
\hline & DELAWARE RIVER BASIN --Continued & & & & & & & & & & \\
\hline 273 & Tobyhanna Creek near Tobyhanna, Pa. & 7. 6 & -- & $\cdots$ & & - & - & Aug. $19,3: 30$ a. m. & - & $2,310 \mathrm{D}$ & 304 \\
\hline 274 & Tobyhanna Creek near Blakeslee, $\mathrm{Pa}$. & 118 & -- & May 23, 1942 & - & ae 7,070 & 59.9 & Aug. 18 & - & $335,300 \mathrm{~A}$ & 299 \\
\hline $275 *$ & Lehigh River at Tannery, $\mathrm{Pa}$ & 322 & $1914-55$ & May 22, 1942 & 16.51 & 29,600 & 91.9 & Aug. 19,5 a. $\mathrm{m}$ & 22.2 & $58,300 \mathrm{~A}$ & 181 \\
\hline 276 & Dilldown Creek near Long Pond, $\mathrm{Pa}$. & 2.39 & $1948-55$ & July 10,1952 & 3.195 & 281 & 118 & Aug. 18,7 p. m. & 3.37 & 342 & 143 \\
\hline 277 & Wild Creek at Hatchery, $\mathrm{Pa}$ & 16.8 & $1941-55$ & May 23, 1942 & 6.00 & $\mathrm{v} 2,360$ & 140 & Aug. $18,9: 30$ p.m. & 5.59 & $1,830 \mathrm{~B}$ & 109 \\
\hline 278 & Pohapoco Creek near Parryville, $\mathrm{Pa}$. & 109 & $1940-55$ & May 23, 1942 & 7.42 & 5,300 & 48.6 & Aug. 18, 11:30 p. m. & $6.7 \%$ & $5,250 \mathrm{~A}$ & 48.2 \\
\hline 279 & Aquashicola Creek at Palmerton, $\mathrm{Pa}$. & 76.7 & $1939-55$ & July 10,1945 & 13.63 & 11,700 & 153 & Aug. $19,3: 30$ a. m. & 9.82 & 4,380 & 57.1 \\
\hline $280 *$ & Lehigh River at Walnutport, $\mathrm{Pa}$. & 889 & $1946-55$ & $\begin{array}{l}\text { Dec. } 4,1950 \\
\text { May 23, } 1942\end{array}$ & $\begin{array}{r}12.66 \\
\mathrm{~b} 20.6\end{array}$ & 46,000 & 51.7 & Aug. $19,6: 30$ a. $\mathrm{m}$ & b17.68 & $' 77,800$ & 87.5 \\
\hline 281 & Little Lehigh Creek near Allentown, $\mathrm{Pa}$. & 80.8 & $1945-55$ & $\begin{array}{rr}\text { Aug. } & 18,1946 \\
\text { July } & 9,1935\end{array}$ & $\begin{array}{l}5.14 \\
9.5\end{array}$ & 1,300 & 16.1 & Aug. 18,9 p. m. & 6.17 & 1,880 & 23.3 \\
\hline 282 & Jordan Creek at Allentown, $\mathrm{Pa}$. & 75.8 & $1944-55$ & May 23,1942 & r7.1 & - & - & Aug. $19,4: 30$ a. $\mathrm{m}$. & b 8.00 & $9,520 \mathrm{~A}$ & 126 \\
\hline 283 & Monocacy Creek at Bethlehem, Pa. & 44.5 & $1948-55$ & $\begin{array}{l}\text { July } 28,1951 \\
\text { July } 10,1945\end{array}$ & $\begin{array}{r}4.42 \\
b 9.74\end{array}$ & $\begin{array}{r}419 \\
5,200\end{array}$ & 9,42 & Aug. 19, 5:30 a. m. & 4.68 & 495 & 11.1 \\
\hline 284* & Lehigh River at Bethlehe $\mathrm{m}, \mathrm{Pa}$. & 1,279 & $\begin{array}{l}1902-05 \\
1909-55\end{array}$ & May 23, 1942 & b23.47 & 92,000 & 71.9 & Aug. 19,10 a.m. & 23.38 & 91,300 & 71.4 \\
\hline 285 & $\begin{array}{l}\text { Musconetcong River at outlet of Lake } \\
\text { Hopatcong, N. J. }\end{array}$ & 25.6 & $1928-55$ & Mar. 19, 1936 & 3.17 & 534 & 20.9 & Aug. 20,5 a. $\mathrm{m}$. & 3.85 & r 700 & 27.3 \\
\hline 286 & $\begin{array}{l}\text { Musconetcong River near Hackettstown, } \\
\text { N. J. }\end{array}$ & 70.0 & $1921-55$ & Mar. 12, 1936 & i 5.88 & 1,430 & 20.4 & Aug. 19,4 p. m. & 3.98 & r 2,200 & 31.4 \\
\hline $287 *$ & $\begin{array}{l}\text { Musconetcong River near Bloomsbury, } \\
\text { N. J. }\end{array}$ & 143 & $1921-55$ & Mar. 15, 1940 & 7.55 & $v 5,760$ & 40.3 & Aug. 19,9 a. $\mathrm{m}$. & 6.95 & $4,430 \mathrm{~A}$ & 31.0 \\
\hline
\end{tabular}




\begin{tabular}{|c|c|c|c|c|c|c|c|c|c|c|c|c|}
\hline $288 *$ & Delaware River at Riegelsville, N. J. & 6,328 & $1906-55$ & $\begin{array}{l}\text { Mar. } 19,1936 \\
\text { Oct. } 10,1903\end{array}$ & $\mid \begin{array}{c}32.45 \\
b 35.9\end{array}$ & $\begin{array}{r}210,000 \\
\text { r 275, 000 }\end{array}$ & $\begin{array}{l}33.2 \\
43.5\end{array}$ & Aug. 20,1 a. $\mathrm{m}$. & 38.85 & - & - & \\
\hline 289* & Tahickon Creek near Pipersville, $\mathrm{Pa}$. & 97.4 & $1935-55$ & Aug. 9,1942 & 10.48 & 13,700 & 141 & Aug. 18,12 p. m. & 11.26 & 16,000 & 164 & \\
\hline $290 *$ & Delaware River at Trenton, N. J. & 6,780 & 1913-55 & $\mid \begin{array}{l}\text { Mar. 19, } 1936 \\
\text { Mar. 8, 1904 } \\
\text { Oct. } 11,1903\end{array}$ & $\begin{array}{r}16.66 \\
\mathrm{k} 22.8 \\
20.7\end{array}$ & $\begin{array}{c}227,000 \\
- \\
295,000\end{array}$ & $\begin{array}{c}33.5 \\
- \\
43.5\end{array}$ & Aug. 20, 6-7 a. m. & 20.83 & r 329,000 & 48.5 & \\
\hline 291* & Neshaminy Creek near Langhorne, $\mathrm{Pa}$. & 210 & $1934-55$ & \begin{tabular}{|l} 
July 23,1938 \\
Aug. 23, 1933
\end{tabular} & $\begin{array}{r}15.94 \\
\mathrm{~b} 17.3\end{array}$ & $\begin{array}{r}.24,800 \\
30,000\end{array}$ & $\begin{array}{l}118 \\
143\end{array}$ & Aug. 19,9 a. $\mathrm{m}$. & 22.84 & $49,300 \mathrm{~A}$ & 235 & \\
\hline 292 & Schuylkill River at Pottsville, $\mathrm{Pa}$. & 53.4 & $1943-55$ & $\left|\begin{array}{lr}\text { Nov. } & 25, \\
\text { May } & 1950 \\
& 1942\end{array}\right|$ & $\begin{array}{r}7.9 \\
\text { b8.8 }\end{array}$ & $\begin{array}{l}4,800 \\
6,170\end{array}$ & $\begin{array}{l}89.9 \\
116\end{array}$ & Aug. 18,8 p. m. & 6.33 & 2,760 & 51.7 & \\
\hline 293 & Schuylkill River at Auburn, $\mathbf{P a}$. & $16 \theta$ & $1947-51$ & \begin{tabular}{|lr} 
Nov. & 25, \\
May & 1950 \\
\end{tabular} & $\begin{array}{c}13.19 \\
\text { b15.5 }\end{array}$ & $\begin{array}{l}9,630 \\
-\end{array}$ & $\begin{array}{c}60.2 \\
-\end{array}$ & Aug. 19 & b10.80 & 6,520 & 40.8 & \\
\hline $294 *$ & Little Schuylkill River at Tamaqua, $\mathrm{Pa}$. & 42.9 & $1916-55$ & May 22, 1942 & 7.95 & 4,310 & 100 & Aug. $18,5: 30$ p. m. & b 11.10 & $7,790 \mathrm{~B}$ & 182 & 5 \\
\hline 295 & $\begin{array}{l}\text { Little Schuylkill River at Drehersville, } \\
\text { Pa. }\end{array}$ & 122 & $1947-51$ & $\left|\begin{array}{l}\text { Nov. } 25,1950 \\
\text { Dec. } 4,1950\end{array}\right|$ & $\begin{array}{l}10.96 \\
11.02\end{array}$ & $\begin{array}{l}10,300 \\
10,300\end{array}$ & $\begin{array}{l}84.4 \\
84.4\end{array}$ & Aug. 19 & b 14.76 & 18,200 & 149 & $\begin{array}{l}\forall \\
\forall \\
\end{array}$ \\
\hline 296 & Schuylkill River at Berne, $\mathrm{Pa}$. & 355 & $1947-55$ & \begin{tabular}{||lr} 
Dec. & 4,1950 \\
May & 1942
\end{tabular} & $\mid \begin{array}{c}14.46 \\
\text { b15.0 }\end{array}$ & $\begin{array}{l}24,200 \\
26,900\end{array}$ & $\begin{array}{l}68.2 \\
75.8\end{array}$ & Aug. 19,1 a. m. & 15.73 & 29,400 & 82.8 & 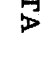 \\
\hline 297 & Tulpehocken Creek near Reading, Pa. & 211 & $|1950-55|$ & |Sept. 1, 1952 & 8.65 & v 7, 680 & 36.4 & Aug. 18, 11:30 p.m. & 7.94 & 6,760 & 32.0 & \\
\hline 298 & Schuylkill River at Pottstown, $\mathrm{Pa}$. & 1,147 & $|1927-55|$ & $\mid$\begin{tabular}{|l} 
May 13,1942 \\
Feb. 28, 1902
\end{tabular} & $\begin{array}{r}20.15 \\
\mathrm{~b} 21.0\end{array}$ & $\begin{array}{l}50,800 \\
53,900\end{array}$ & $\begin{array}{l}44.3 \\
47.0\end{array}$ & Aug. $19,1: 30$ p. m. & 17.98 & 42,300 & 36.9 & \\
\hline 299 & Perkiomen Creek at Graterford, $\mathrm{Pa}$. & 279 & $|1914-55|$ & |July 9,1935 & 18.26 & 39,900 & 143 & Aug. 19, 12:15 a.m. & 14.08 & 24,400 & 87.5 & \\
\hline 300 & Schuylkill River at Philadelphia, Pa. & 1,893 & $\left|\begin{array}{c}1898- \\
1912 \\
1931-55\end{array}\right|$ & $\mid \begin{array}{ll}\text { Mar. } & 1,1902 \\
\text { Oct. } & 4,1869\end{array}$ & $\begin{array}{r}14.8 \\
\mathrm{~b} 17.0\end{array}$ & $\begin{array}{r}98,000 \\
135,000\end{array}$ & $\begin{array}{l}51.8 \\
71.3\end{array}$ & Aug. $19,6: 30$ a. m. & 14.32 & 89,800 & 47.4 & \\
\hline 301 & Ridley Creek at Maylan, $\mathbf{P a}$. & 31.9 & $1931-53$ & |Nov. 25,1950 & 10.84 & 5,720 & 179 & Aug. 18 & b9. 42 & 4,390 & 138 & \\
\hline 302 & Chester Creek near Chester, Pa. & 61.1 & $1931-55$ & $\mid$ Nov. 25,1950 & b16.21 & v 14,400 & 236 & Aug. 18,9 p. m. & b 13.57 & $9,380 \mathrm{~B}$ & 154 & \\
\hline 303 & $\begin{array}{l}\text { Brandywine Creek at Chadds Ford, Pa. } \\
\text { SUSQUEHANNA RIVER BASIN }\end{array}$ & 287 & $\left|\begin{array}{lll}1911 & -53\end{array}\right|$ & Mar. 5, 1920 & b15.0 & 17,200 & 59.9 & Aug. 19,2 a.m. & b 14.64 & 16,400 & 57.1 & \\
\hline
\end{tabular}


Table 2. --Summary of stages and discharges in the Northeast during the floods of August 1955--Continued

\begin{tabular}{|c|c|c|c|c|c|c|c|c|c|c|c|c|}
\hline \multirow{2}{*}{$\begin{array}{l}\text { No. } \\
\text { on } \\
\text { pl. } \\
1\end{array}$} & \multirow[b]{2}{*}{ Stream and place of determination } & \multirow{2}{*}{$\begin{array}{c}\text { Drainage } \\
\text { area } \\
\text { (sq mi) }\end{array}$} & \multirow{2}{*}{$\begin{array}{l}\text { Period } \\
\text { of } \\
\text { record }\end{array}$} & \multicolumn{5}{|c|}{ Maximum flood previously known } & \multicolumn{4}{|c|}{ Maximum during present flood } \\
\hline & & & & \multicolumn{2}{|r|}{ Date } & $\begin{array}{l}\text { Gage } \\
\text { height } \\
\text { (feet) }\end{array}$ & $\begin{array}{c}\text { Discharge } \\
\text { (cfs) }\end{array}$ & $\begin{array}{l}\text { Cfs per } \\
\text { sq mi }\end{array}$ & Date and hour & $\begin{array}{l}\text { Gage } \\
\text { height } \\
\text { (feet) }\end{array}$ & $\begin{array}{c}\text { Discharge } \\
\text { (cfs) }\end{array}$ & $\begin{array}{l}\text { Cfs per } \\
\text { sq mi }\end{array}$ \\
\hline 305 & $\begin{array}{l}\text { SUSQUEHANNA RIVER BASIN--Con. } \\
\text { Lackawanna River at Archbald, } \mathrm{Pa} \text {. }\end{array}$ & 108 & $1940-55$ & \multicolumn{2}{|c|}{ May 22, 1942} & 10.58 & 9,510 & 88.1 & Aug. 19,1 a. $\mathrm{m}$. & 7.50 & 5,050 & 46.8 \\
\hline 306 & Roaring Brook at Dunmore, $\mathrm{Pa}$. & 49.4 & --- & \multicolumn{2}{|r|}{--} & - & - & - & Aug. 19,1 a. $\mathrm{m}$. & - & $18,500 \mathrm{D}$ & 374 \\
\hline 307 & Little Roaring Brook at Dunmore, $\mathrm{Pa}$. & 5.12 & -- & \multicolumn{2}{|r|}{$-\cdot$} & - & - & - & Aug. 18 & - & 601D & 117 \\
\hline 308 & Spring Brook at Rockdale, $\mathrm{Pa}$. & 15.9 & -- & May & 1942 & - & 2,120 & 133 & Aug. 18-19 & - & $3,130 \mathrm{D}$ & 197 \\
\hline 309 & Spring Brook near Rockdale, $\mathrm{Pa}$. & 37.0 & -- & May & 1942 & - & 7,500 & 203 & Aug. 19 & - & $9,280 \mathrm{D}$ & 251 \\
\hline 310 & $\begin{array}{l}\text { Spring Brook at intake Dam near } \\
\text { Moosic, } \mathrm{Pa} \text {. }\end{array}$ & 43.1 & --- & May & 1942 & - & 8,360 & 194 & Aug. 19 & - & $10,500 \mathrm{D}$ & 244 \\
\hline $312 *$ & Lackawanna River at Old Forge, $\mathrm{Pa}$. & 332 & $1938-55$ & May & 23,1942 & 15.30 & 20,900 & 63.0 & Aug. 19,2 a. $\mathrm{m}$. & 20.05 & $31,000 \mathrm{~A}$ & 93.4 \\
\hline 313 & Susquehanna River at Wilkes-Barre, Pa. & 9,860 & $\begin{array}{r}1890- \\
1955\end{array}$ & Mar. & 20,1936 & 33.07 & 232,000 & 23. 3 & Aug. 19, 7:30 a. m. & 12.84 & 52,500 & 5.27 \\
\hline 314 & Toby Creek at Luzerne, $\mathrm{Pa}$. & 32.4 & $1941-55$ & Dec. & 30,1942 & - & - & - & Aug. 18, 7 p. m. & 4.33 & 2,440 & 75.3 \\
\hline 315 & Solomon Creek at Wilkes-Barre, Pa. & 15.7 & $1940-55$ & $\begin{array}{l}\text { Aug. } \\
\text { Sept. }\end{array}$ & $\begin{array}{l}17,1947 \\
.16,1933\end{array}$ & $\begin{array}{r}6.40 \\
\mathrm{~b} 11.4\end{array}$ & $\begin{array}{l}- \\
-\end{array}$ & $\begin{array}{l}- \\
-\end{array}$ & Aug. 18, 7:30 p.m. & 9.83 & $2,450 \mathrm{C}$ & 157 \\
\hline $316 *$ & Wapwallopen Creek near Wapwallopen, $\mathrm{Pa}$. & 45.8 & $1919-55$ & July & 22,1952 & 8.96 & 2,980 & 65.1 & Aug. 18,11 p. m. & 9.23 & 3,140 & 68.6 \\
\hline 317 & Nescopeck Creek near Nescopeck, Pa. & 161 & -- & & -- & - & - & - & Aug. 18-19 & - & $13,400 \mathrm{~B}$ & 83.2 \\
\hline 318 & Fishing Creek near Bloomsburg, $\mathrm{Pa}$. & 274 & $1938-55$ & $\begin{array}{l}\text { Mar } \\
\text { Mar. }\end{array}$ & $\begin{array}{r}31,1940 \\
.18,1936\end{array}$ & $\begin{array}{r}\text { b } 12.08 \\
11.86\end{array}$ & $\begin{array}{l}18,100 \\
17,600\end{array}$ & $\begin{array}{l}66.1 \\
64.2\end{array}$ & Aug. 19,4 a. m. & 8.54 & 7,980 & 29.1 \\
\hline
\end{tabular}

(




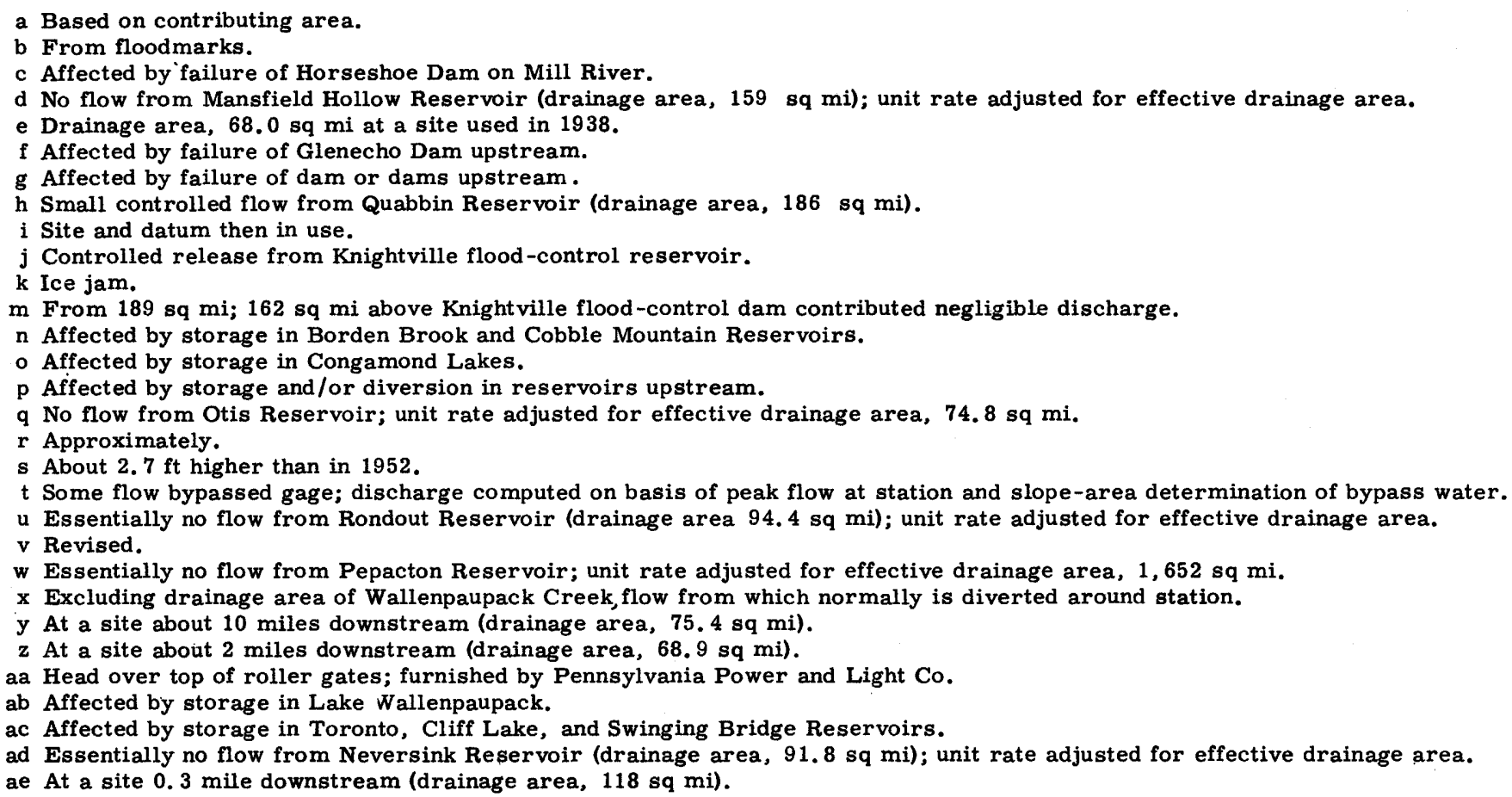

(nelease from Knightville flood-control reservoir.

$\mathrm{m}$ From $189 \mathrm{sq} \mathrm{mi} ; 162 \mathrm{sq} \mathrm{mi}$ above Knightville flood-control dam contributed negligible discharge.

n Affected by storage in Borden Brook and Cobble Mountain Reservoirs.

q No flow from Otis Reservoir; unit rate adjusted for effective drainage area, $74.8 \mathrm{sq} \mathrm{mi}$

r Approximately.

Some flow bypassed gage; discharge computed on basis of peak flow at station and slope-area determination of bypass water.

u Essentially no flow from Rondout Reservoir (drainage area $94.4 \mathrm{sq} \mathrm{mi}$ ); unit rate adjusted for effective drainage area.

At a site about 2 miles downstream (drainage area, $68.9 \mathrm{sq} \mathrm{mi)}$.

ab Affected by storage in Lake Wallenpaupack.

ac Affected by storage in Toronto, Cliff Lake, and Swinging Bridge Reservoirs.

ae At a site 0.3 mile downstream (drainage area, $118 \mathrm{sq} \mathrm{mi}$ ). 
In figures 4 and 5 , the unit discharge (cubic feet per second per square mile) has been plotted against drainage area (square miles) for all stations listed in the summary of stages and discharges (table 2). These graphs, show the interrelation of the peak discharges at the many observation points. An excellent idea of the upper limiting parameter of the plotted points may be obtained from the graphs, and they serve as a basis for comparison of the data for the present flood with those for other floods. The reference numbers used in table 2 and on plate 1 are shown alongside the highest plotted points for identity.

\section{Gaging-Station Records}

Continuous records are collected at the regular gaging stations that show both stage and discharge at any moment and their variations during any length of time. It is thus possible to plot graphs of discharge in flood periods, like those shown in figures 6,7 , and 8. These $g$ raphs are for data at selected gaging stations in the August flood area, chosen for their geographic distribution. The peak discharze of the highest previous flood at each station has been shown as a point of comparison.

The graphs show the different flow characteristics of the streams and the relative effect on them of the Connie and Diane storms. The Connie storm had very little effect on some streams, but it had large effect on others. For most stations, the hydrograph in the Connie storm period (around August 12 and 13) was drawn from daily mean discharges. This has the effect of attenuating the rise at that time as compared with the more detailed graph for the later and greater peak. The area under the curve, however, essentially represents the volume of flow.
One of the more interesting graphs is that for Charles River in figure 6. Charles River rose to an intermediate peak and leveled for a while. This was followed by a slower rise to a higher peak, followed by a quite slow recession. The action demonstrates the streamflow characteristic of the basin which is formed by sandy soils that have high rainfall absorption, flat terrain and its slow runoff characteristics, and a sizable area of ponds. It is quite in contrast with the hydrograph for a number of the other stations where the water rose quickly and then subsided almost as quickly.

In order to plot detailed graphs of stage and dis charge, and to present other information for the floods, tabulations of data are presented in the following pages for 51 gaging stations in the flood area. This is a selection from a total of about 200 gazing stations in that area. The number shown in parentheses with the name of each gaging station is the same as that used for that station in the summary cable 2 and on the map showing the location of all sites (plate 1).

For each gaging station the tabulations include a station description and two tables. The descriptive section contains a description of the location, how the record was obtained, maxima during the present and during previous floods, and other information. The first table lists the daily mean discharge during the flood period, usually August 1-31. The second table shows stage and discharge in sufficient detail to plot accurate hydrographs. In most cases, the daily mean discharge can be computed from the detailed data; in cases where this cannot be done, because of minor variations of no hydrologic import, the fact is noted.

Other, and more detailed, data are available in the basic records held by the Geological Survey, and these may be studied at the appropriate field office. 


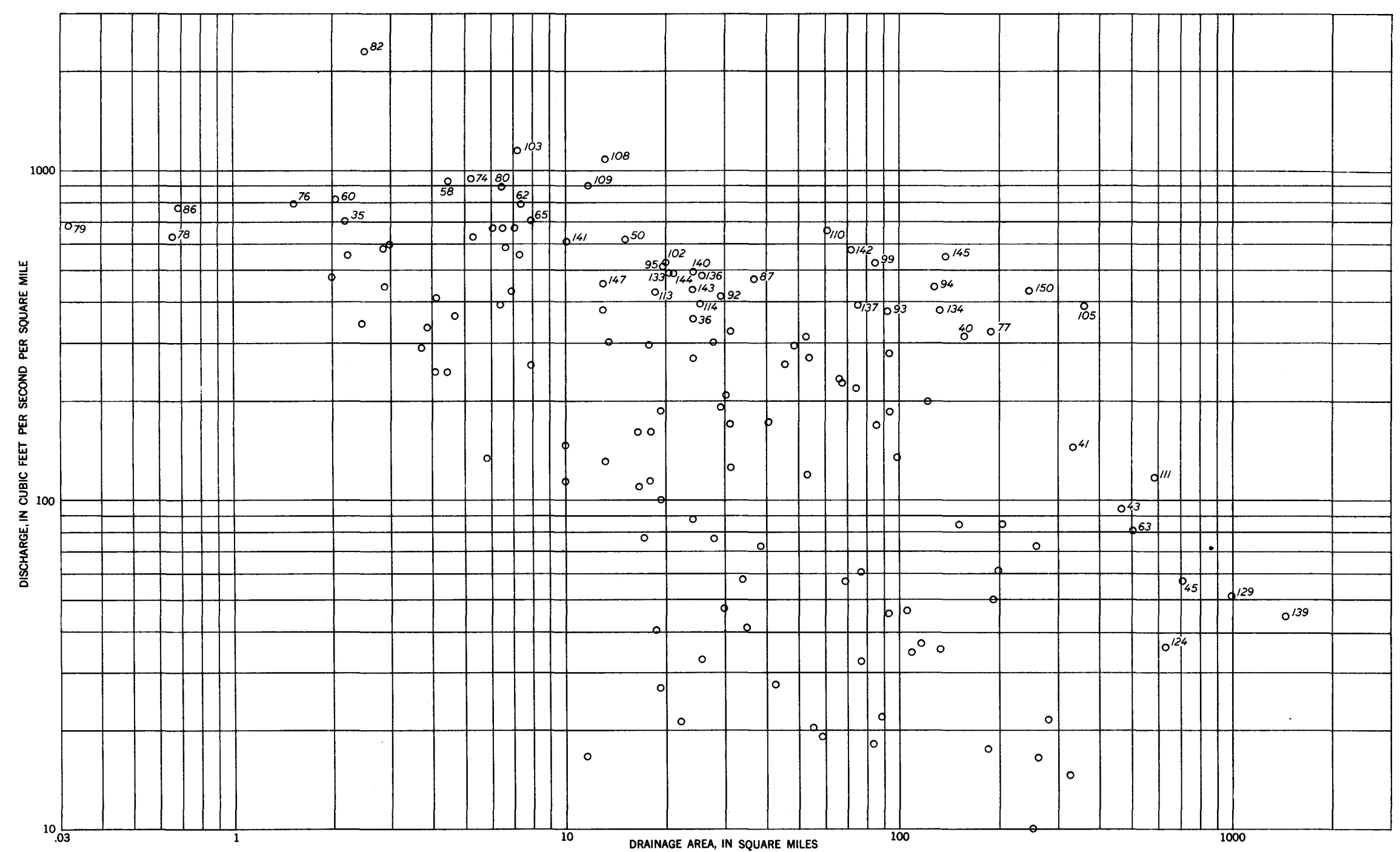




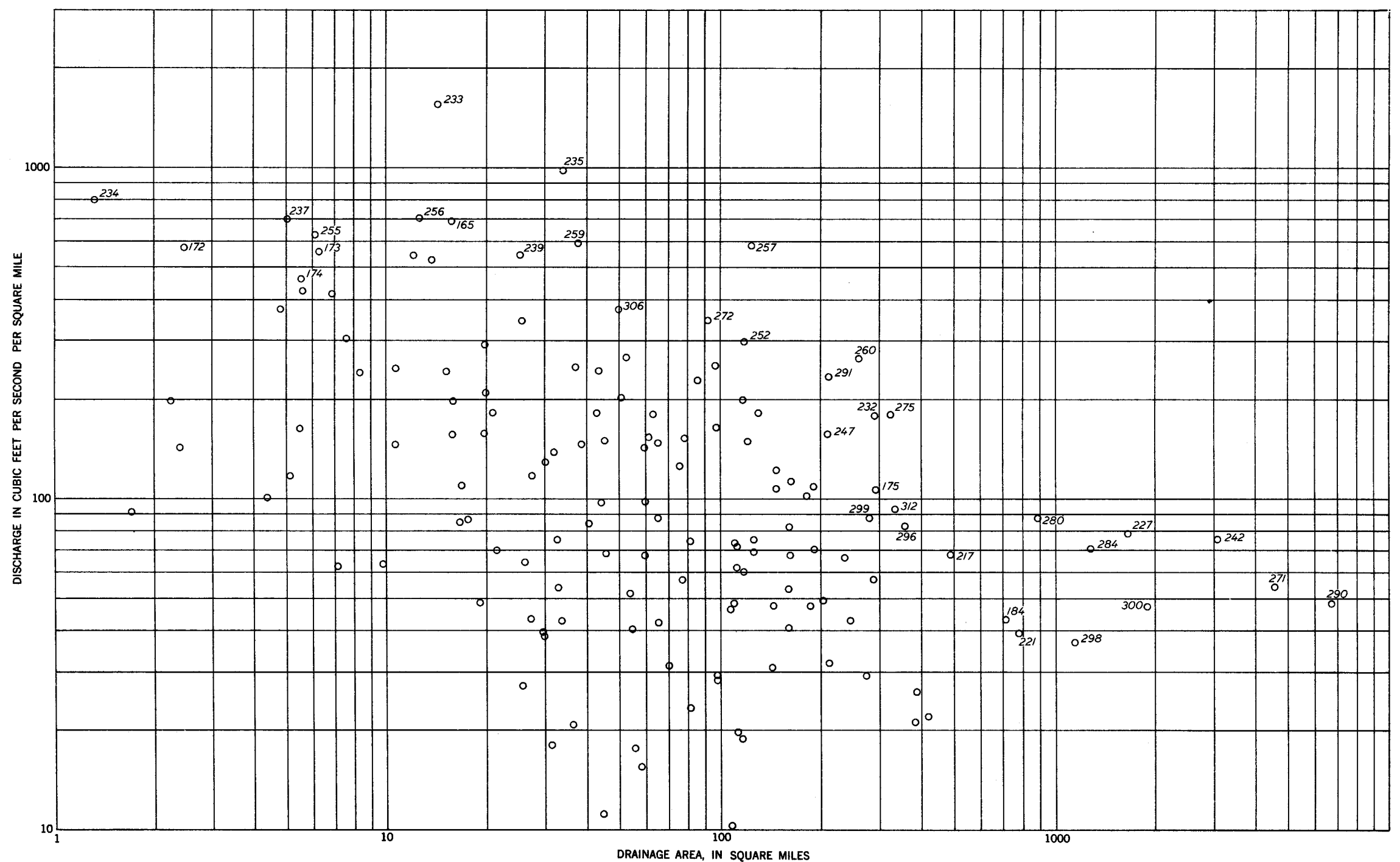

Figure 5. --Graph of relation of unit discharge and size of drainaze area, west of Hudson River, floods of August 18-19, 1955. 

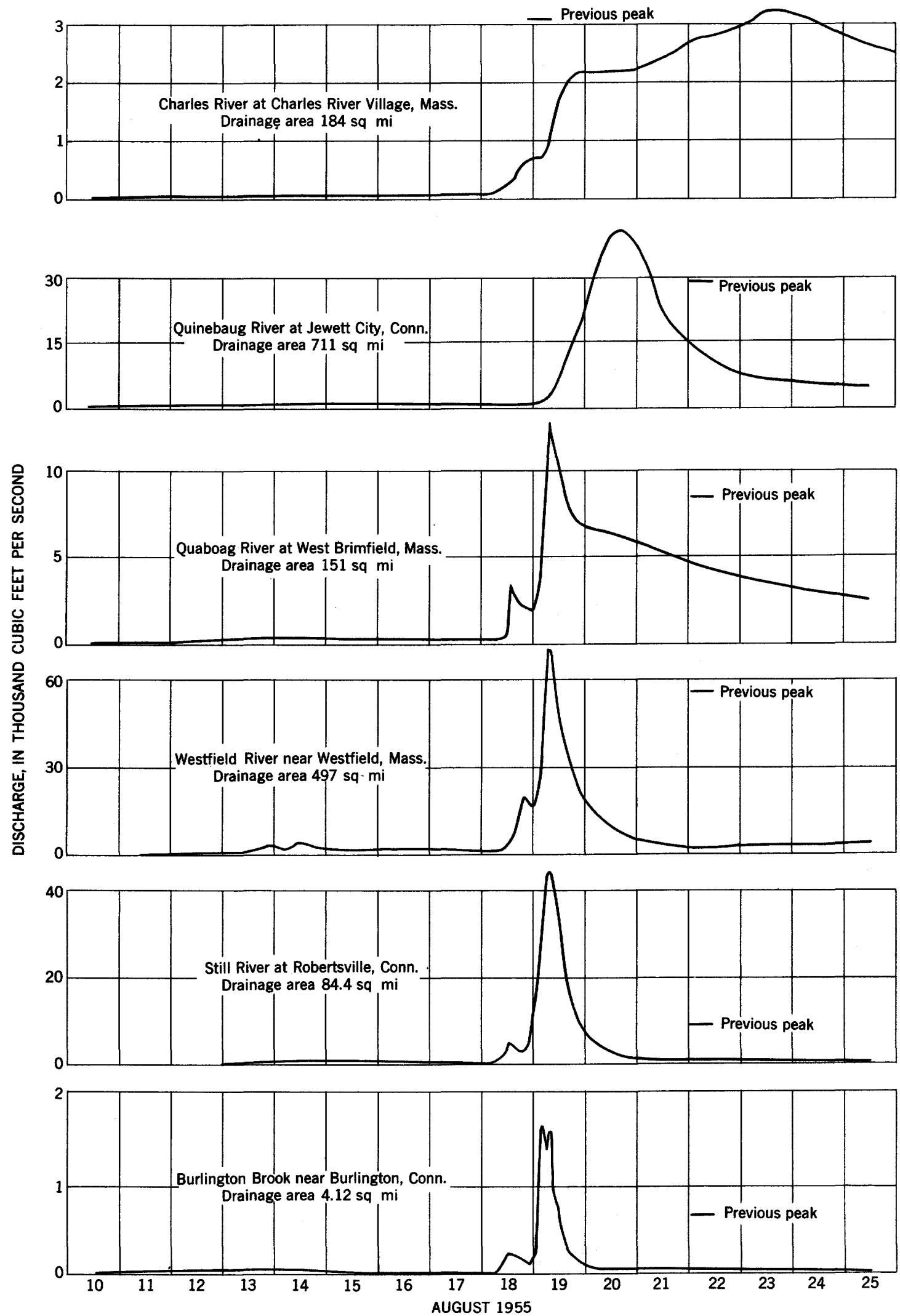

Figure 6. --Graphs of discharge at selected gaging stations, August 10-25. 

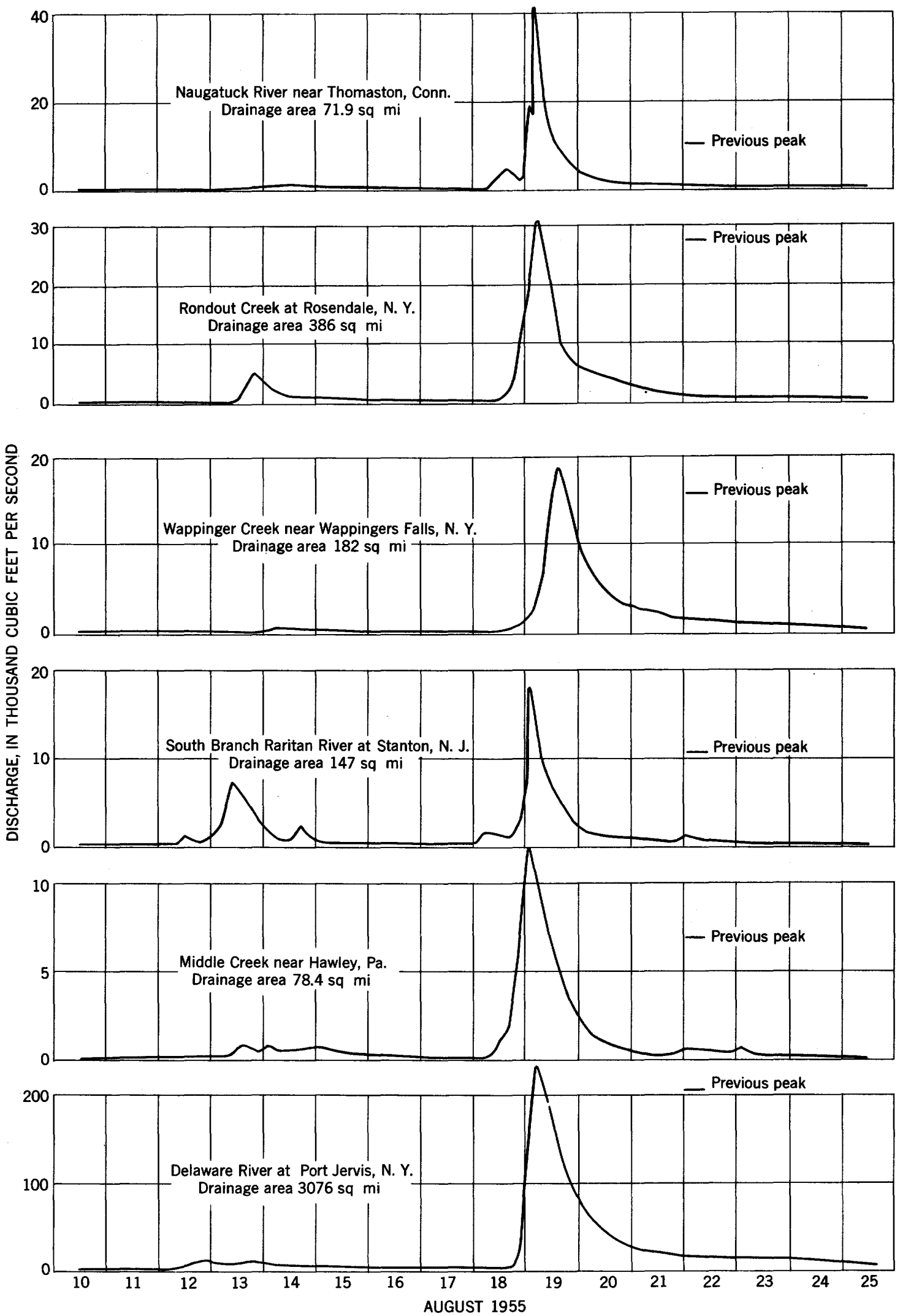

Figure 7. --Graphs of discharge at selected gaging stations, August 10-25. 

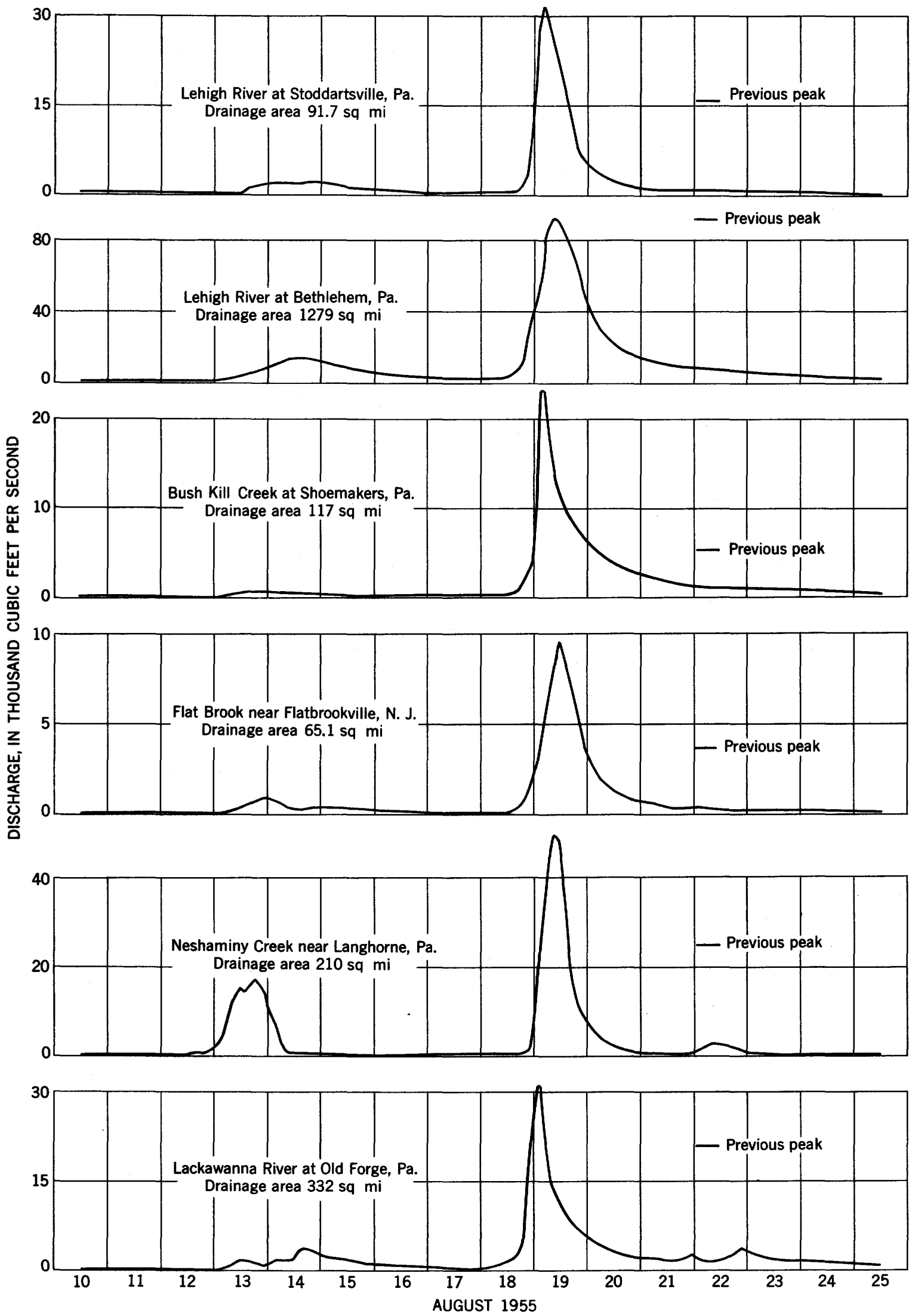

Figure 8. --Graphs of discharge at selected gaging stations, August 10-25. 
(2) Concord River below River Meadow Brook, at Lowell, Mass.

Location.--Lat $42^{\circ} 38^{\prime} 12^{\prime \prime}$, long $71^{\circ} 18^{\prime} 09^{\prime \prime}$, on right bank $300 \mathrm{ft}$ downstream from Rogers Street Bridge at Lowell, Middlesex County, 0.3 mile downstream' from River Meadow Brook, and 0.8 mile upstream from mouth. Datum of gage is $67.41 \mathrm{ft}$ above mean sea level, datum of 1929 .

Drainage area.--Total above gage, $405 \mathrm{sq} \mathrm{mi}$; net above gage, $312 \mathrm{sq} \mathrm{ml}$ (diversion as needed from $92.6 \mathrm{sq} \cdot \mathrm{mi}$ for use of Boston metropolitan district)

Gage-he1ght record.--Water-stage recorder graph.

Discharge record.--Stage-discharge relation defined by current-meter measurements. Backwater from grass at times. Maxjma.-August 1955: Discharge, $4,540 \mathrm{cfs} 8$ to $10 \mathrm{p.m}$. Aug 23 (gage he1ght, $8.97 \mathrm{ft}$ ).

1936 to July 1955: Discharge, 3,790 cfs July 29,1938 (gage he1ght, 8.11 ft).

Remarks.--Discharge includes water wasted from $92.6 \mathrm{sq} \mathrm{mi}$ in basins of Sudbury River and Lake Cochituate. Water

diverted above station for use of city of Lowell. Medium and low flows regulated by mills above station.

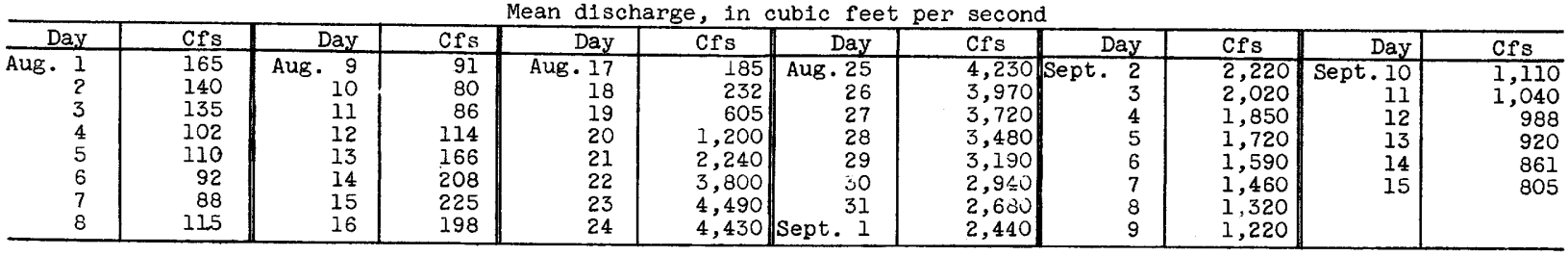

Gage height, in feet, and discharge, in cubic feet per second, at indicated time

\begin{tabular}{|c|c|c|c|c|c|c|c|c|c|c|c|}
\hline Time & $\begin{array}{c}\text { Gage } \\
\text { height }\end{array}$ & $\begin{array}{c}\text { Dis- } \\
\text { charge }\end{array}$ & Time & $\begin{array}{c}\text { Gage } \\
\text { he1ght }\end{array}$ & $\begin{array}{c}\text { D1s- } \\
\text { charge }\end{array}$ & TIme & $\begin{array}{c}\text { Gage } \\
\text { helght }\end{array}$ & $\begin{array}{l}\text { Dis- } \\
\text { charge }\end{array}$ & Time & $\begin{array}{c}\text { Gage } \\
\text { he1ght }\end{array}$ & $\begin{array}{l}\text { D1s- } \\
\text { charge }\end{array}$ \\
\hline 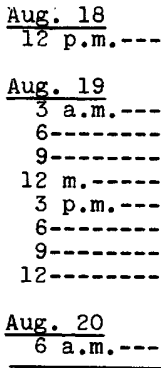 & $\begin{array}{l}5.23 \\
5.39 \\
5.45 \\
5.84 \\
6.05 \\
6.12 \\
6.19 \\
6.25\end{array}$ & $\begin{array}{l}297 \\
375 \\
408 \\
644 \\
784 \\
840 \\
890 \\
935\end{array}$ & 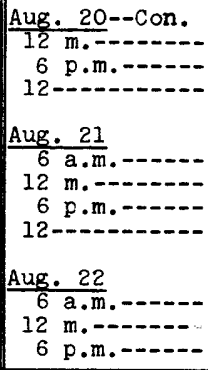 & $\begin{array}{l}7.27 \\
7.28 \\
7.85 \\
8.19\end{array}$ & $\begin{array}{l}1,190 \\
1,310 \\
1,510 \\
1,840 \\
2,190 \\
2,600 \\
3,170 \\
3,540 \\
3,860 \\
4,070\end{array}$ & 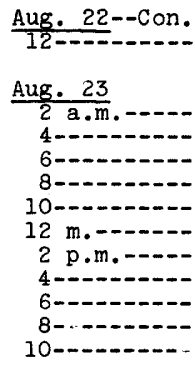 & $\begin{array}{l}8.84 \\
3.88 \\
8.92 \\
8.95 \\
8.96 \\
8.96 \\
8.96 \\
8.95 \\
8.95 \\
8.97 \\
8.97\end{array}$ & $\begin{array}{l}4,280 \\
\\
4,320 \\
4,410 \\
4,480 \\
4,520 \\
4,530 \\
4,530 \\
4,530 \\
4,520 \\
4,520 \\
4,540 \\
4,540\end{array}$ & 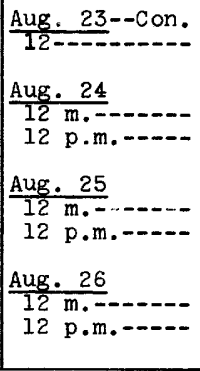 & $\begin{array}{l}8.73 \\
8.63\end{array}$ & $\begin{array}{l}4,230 \\
4,100\end{array}$ \\
\hline
\end{tabular}

(3) Charles River at Charles River Village, Mass.

Location.--Lat $42^{\circ} 15^{\prime} 23^{\prime \prime}$, long $71^{\circ} 15^{\prime} 42^{\prime \prime}$, on right bank 0.25 mile downstream from highway bridge at Charles River V11lage, Norfolk County, $0.8 \mathrm{mile}$ downstream from Noanet Brook, and 1.3 miles northeast of Dover. Datum of

gage is $89.76 \mathrm{ft}$ above mean sea level, datum of 1929.

Drainage area. $--184 \mathrm{sq} \mathrm{ml}$.

Gage-helght record.--Water-stage recorder graph.

Discharge record.-Stage-discharge relation defined by current-meter measurements.

Maxima.-August 1955: Discharge, 3,220 cfs 2 to 3 p.m. Aug. 23 (gage he1ght, 9.24 ft)

1937 to July 1955: Discharge, 3,110 cfs July 27, 1938 (gage height, 9.00 ft).

Flood in March 1936 reached a discharge of 3,170 cfs, by computation of flow over dam at s1te a quarter of a mile above station.

Remarks.-Diversion above station for municipal supply of Wellesley and Needham. Occasional diversion from Sudbury River basin.

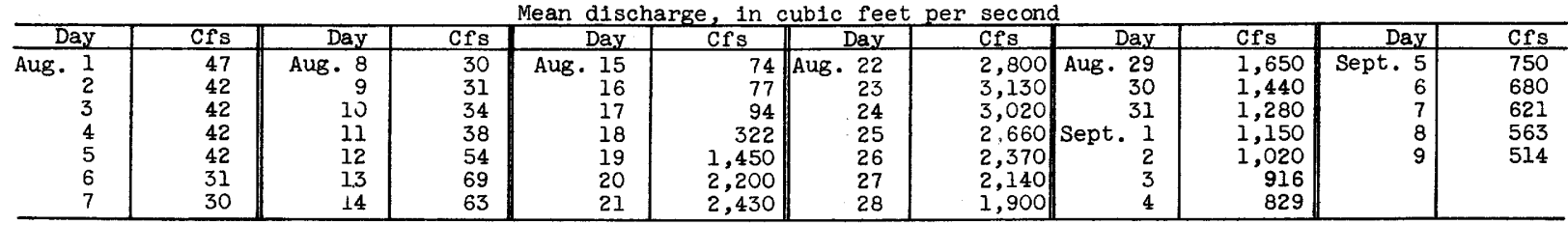


(3) Charles River at Charles River Village, Mass.--Continued

Gage height, in feet, and discharge, in cubic feet per second, at indicated time

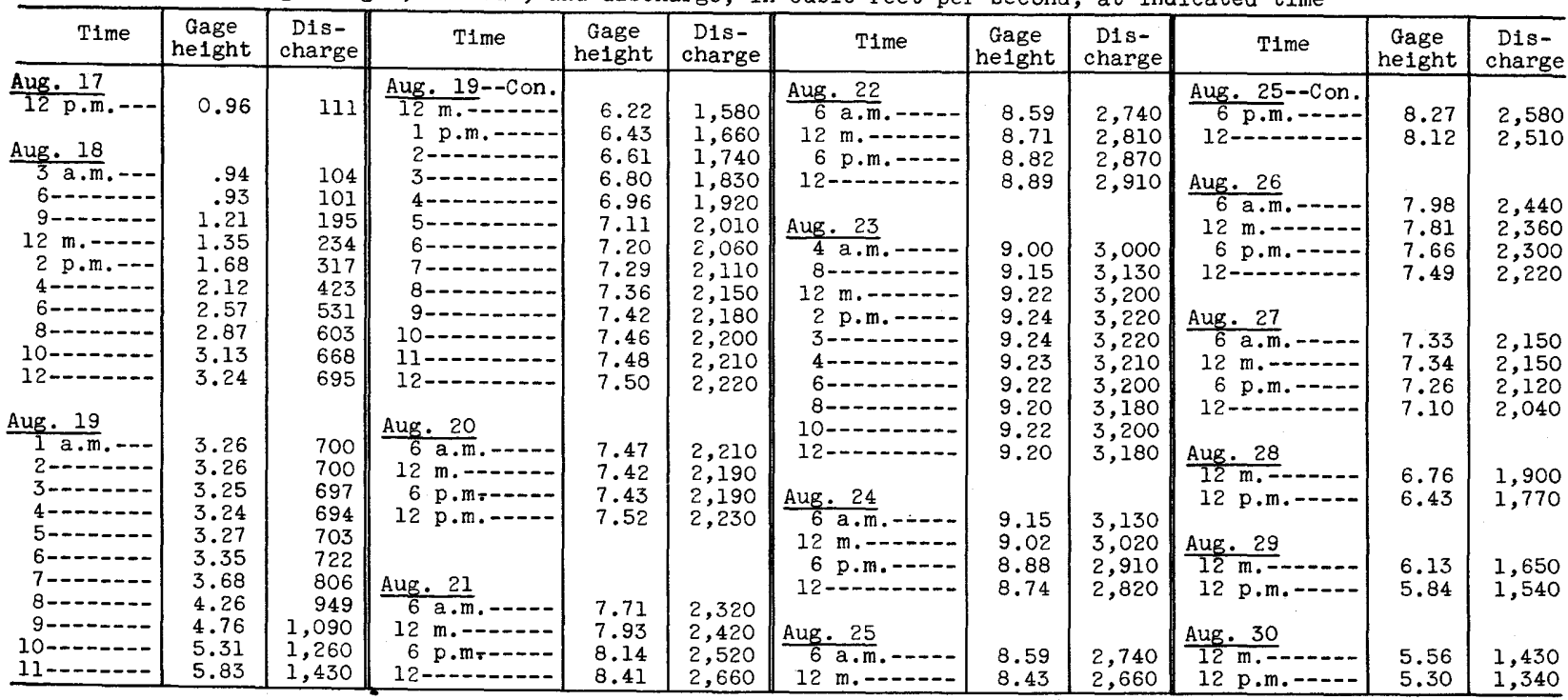

(16) Blackstone River at Woonsocket, R. I.

Location.--Lat $42^{\circ} 00^{\prime} 22^{\prime \prime}$, long $71^{\circ} 30^{\prime} 13^{\prime \prime}$, on right bank at Woonsocket, Providence County, 50 ft downstream from Peters River. Datum of gage is $107.42 \mathrm{ft}$ above mean sea level, datum of 1929 .

Drainage area. $-416 \mathrm{sq} \mathrm{mi}$.

Gage-height record.--Water-stage recorder graph except for periods 8:30 p.m. Aug. 19 to 1 p.m. Aug. 22 and 6:30 p.m. Aug. 23 to $8 \mathrm{am}$. Aug. 26. One measurement of stage from a temporary reference point obtained Aug. 20 . Graph for Aug. 24, 25 drawn on basis of range line and trend of recorded graph.

Discharge record.--Stage-discharge relation defined by current-meter measurements below 15,000 cfs and extended to peak stage on basis of slope-area determination at gage height 21.8 ft. Discharge for period 9 p.m. Aug. 19 to noon Aug. 22 estimated on basis of 1 measurement of stage, floodmarks, and records for Blackstone River at Northbridge, Mass. Backwater from grass at times.

axima.--August 1955: Discharge, 32,900 cfs 10 p.m. Aug. 19 (gage height, $21.8 \mathrm{ft}$, from floodmarks), affected by ailure of Horseshoe Dam on Mill River.

1929 to July 1955: Discharge, 15,100 cfs July 24, 1938 (gage height, $14.43 \mathrm{ft}$ ).

Remarks.--Discharge includes flow diverted from Nashua River basin to Blackstone River basin for supply of Worcester, Mass., and flow diverted around station in Hamlet Trench. Flood flow affected by reservolrs and by dam failures above station. Low and medium flows regulated by powerplants above station.

Mean discharge, in cuble feet per second, August 1955

\begin{tabular}{c|c|c|c||r|r|r|r|r|r|r|r|r|}
\hline Day & Cfs & Day & Cfs & Day & Cfs & Day & Cfs & Day & Cfs & Day & Cfs \\
\hline 1 & 142 & 7 & 128 & 12 & 211 & 17 & 318 & 22 & 6,430 & 27 & 2,190 \\
2 & 162 & 8 & 150 & 13 & 357 & 18 & 686 & 23 & 4,410 & 28 & 1,910 \\
3 & 135 & 9 & 185 & 14 & 595 & 19 & 11,200 & 24 & 4,260 & 29 & 1,710 \\
4 & 125 & 10 & 150 & 15 & 403 & 20 & 25,900 & 25 & 3,500 & 30 & 1,460 \\
5 & 139 & 11 & 155 & 16 & 348 & 21 & 12,200 & 26 & 2,750 & 31 & 1,380 \\
6 & 127 & & & & & & \\
\hline
\end{tabular}

Gage height, in feet, and discharge, in cubic feet per second, at indicated time

\begin{tabular}{|c|c|c|c|c|c|c|c|c|c|c|c|}
\hline Time & $\begin{array}{l}\text { Gage } \\
\text { height }\end{array}$ & $\begin{array}{l}\text { Dis- } \\
\text { charge }\end{array}$ & Time & $\begin{array}{c}\text { Gage } \\
\text { height }\end{array}$ & $\begin{array}{l}\text { Dis- } \\
\text { charge }\end{array}$ & Time & & $\begin{array}{c}\text { Dis- } \\
\text { charge }\end{array}$ & Time & & $\begin{array}{l}\text { Dis- } \\
\text { charge }\end{array}$ \\
\hline 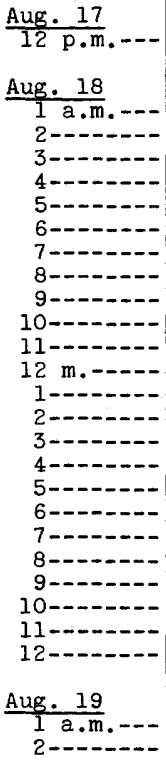 & $\begin{array}{l}2.50 \\
2.51 \\
2.51 \\
2.50 \\
2.48 \\
2.84 \\
3.12 \\
2.76 \\
2.88 \\
2.88 \\
2.98 \\
3.41 \\
3.22 \\
3.80 \\
3.52 \\
3.55 \\
3.60 \\
3.61 \\
4.22 \\
4.36 \\
4.19 \\
4.20 \\
4.23 \\
4.33\end{array}$ & $\begin{array}{r}291 \\
294 \\
294 \\
291 \\
285 \\
407 \\
530 \\
377 \\
423 \\
423 \\
465 \\
683 \\
580 \\
939 \\
751 \\
770 \\
803 \\
810 \\
1,230 \\
1,340 \\
1,210 \\
1,220 \\
1,240 \\
1,320\end{array}$ & 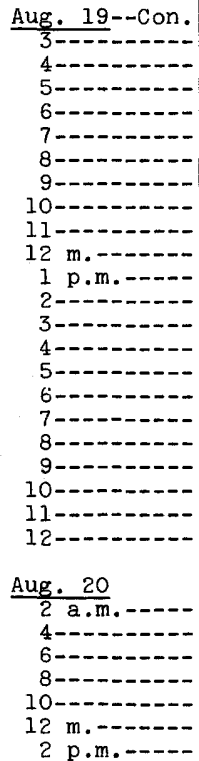 & $\begin{array}{r}4.63 \\
4.86 \\
5.70 \\
6.89 \\
7.51 \\
8.09 \\
8.53 \\
9.20 \\
9.89 \\
10.28 \\
10.64 \\
11.47 \\
12.17 \\
12.77 \\
13.47 \\
14.21 \\
14.69 \\
15.37 \\
--- \\
21.8 \\
--- \\
-.- \\
\\
--- \\
--- \\
--- \\
--- \\
--- \\
---\end{array}$ & $\begin{array}{l}1,650 \\
1,900 \\
2,910 \\
4,460 \\
5,260 \\
6,010 \\
6,580 \\
7,460 \\
8,390 \\
8,940 \\
9,440 \\
10,600 \\
11,700 \\
12,600 \\
13,600 \\
14,700 \\
15,500 \\
16,600 \\
27,100 \\
32,900 \\
31,500 \\
29,800 \\
\\
28,400 \\
27,800 \\
27,800 \\
28,400 \\
29,200 \\
29,600 \\
28,300\end{array}$ & 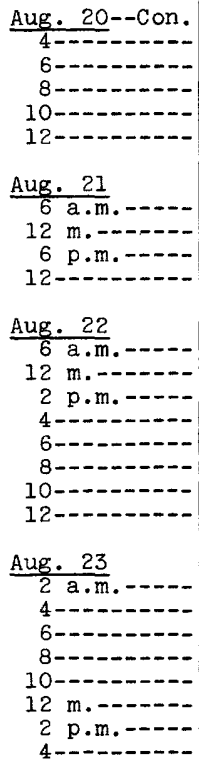 & $\begin{array}{l}--- \\
--- \\
8.05 \\
7.84 \\
7.68 \\
7.52 \\
7.35 \\
7.18 \\
\\
7.01 \\
6.85 \\
6.99 \\
6.81 \\
6.74 \\
6.71 \\
6.71 \\
6.72\end{array}$ & $\begin{array}{c}25,200 \\
22,700 \\
20,600 \\
19,100 \\
17,500 \\
\\
14,200 \\
11,800 \\
9,940 \\
8,470 \\
\\
7,320 \\
6,310 \\
5,960 \\
5,680 \\
5,480 \\
5,270 \\
5,050 \\
4,830 \\
\\
4,620 \\
4,420 \\
4,590 \\
4,380 \\
4,290 \\
4,260 \\
4,260 \\
4,270\end{array}$ & 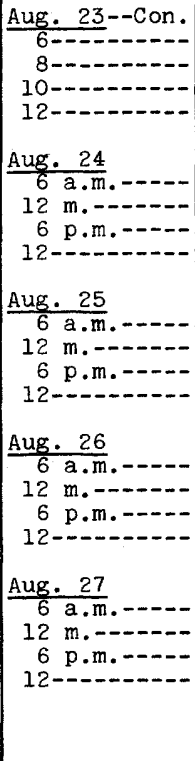 & $\begin{array}{l}6.88 \\
6.73 \\
6.57 \\
6.40 \\
6.24 \\
6.09 \\
5.92 \\
5.72\end{array}$ & $\begin{array}{l}4,300 \\
4,400 \\
4,470 \\
4,530 \\
\\
4,460 \\
4,280 \\
4,080 \\
3,880 \\
\\
3,690 \\
3,510 \\
3,310 \\
3,090 \\
\end{array}$ \\
\hline
\end{tabular}


(20) Willimantic River near South Coventry, Conn.

Location.--Lat $41^{\circ} 45^{\prime} 02^{\prime \prime}$, long $72^{\circ} 15^{\prime} 58^{\prime \prime}$, on left bank 700 ft upstream from highway bridge, 1 mile downstream from Mill Brook. 2.4 miles southwest of South Coventry, Tollard County, 2.8 miles upstream from Hop River, and at mile 6.3. Datum of gage is $239.05 \mathrm{ft}$ above mean sea level, datum of 1929 (levels by Corps of Engineers). Drainage area. - -121 sq $\mathrm{mi}$.

Gage-helght record. - Water-stage recorder graph except for period 1:30 to $10 \mathrm{p.m}$. Aug. 19. Graph for this per1od drawn on basis of floodmark and time of peak.

Discharge record.--Stage-discharge relation defined by current-meter measurements below 3,600 cfs and by computation of flow over dam at gage height $12.19 \mathrm{ft}$ and contracted-opening determination at gage height $18.66 \mathrm{ft}$.

Maxima.--August 1955: Discharge, $24,200 \mathrm{cfs} 4 \mathrm{p} . \mathrm{m}$. Aug. 19 (gage helght, $18.66 \mathrm{ft}$, from floodmarks).

1931 to July 1955: Discharge, $15,500 \mathrm{cfs}$ Sept. 21 , 1938 (gage height, 18.08 ft, from floodmarks), by compu-

tation of flow over dam at Eagleville above station, prior to washing out, adjusted for flow from intervening area.

Remarks.--Flood flow affected brlefly when storage was released by washout of dam at Eagleville.

Mean discharge, in cubic feet per second, August 1955

\begin{tabular}{r|r|r|r|r|r|r|r||r|r|r||r}
\hline Day & Cfs & Day & Cfs & Day & Cfs & Day & Cfs & Day & Cfs & Day & Cfs \\
\hline 1 & 24 & 7 & 36 & 12 & 147 & 17 & 186 & 22 & 1,030 & 27 & 408 \\
2 & 25 & 8 & 73 & 13 & 735 & 18 & 337 & 23 & 924 & 28 & 392 \\
3 & 27 & 9 & 60 & 14 & 1,230 & 19 & 12,100 & 24 & 830 & 29 & 336 \\
4 & 29 & 10 & 53 & 15 & 628 & 20 & 6,650 & 25 & 580 & 30 & 288 \\
5 & 30 & 11 & 42 & 16 & 310 & 21 & 1,850 & 26 & 468 & 31 \\
6
\end{tabular}

Gage height, in feet, and disciarge, in cubic feet per second, at indicated time

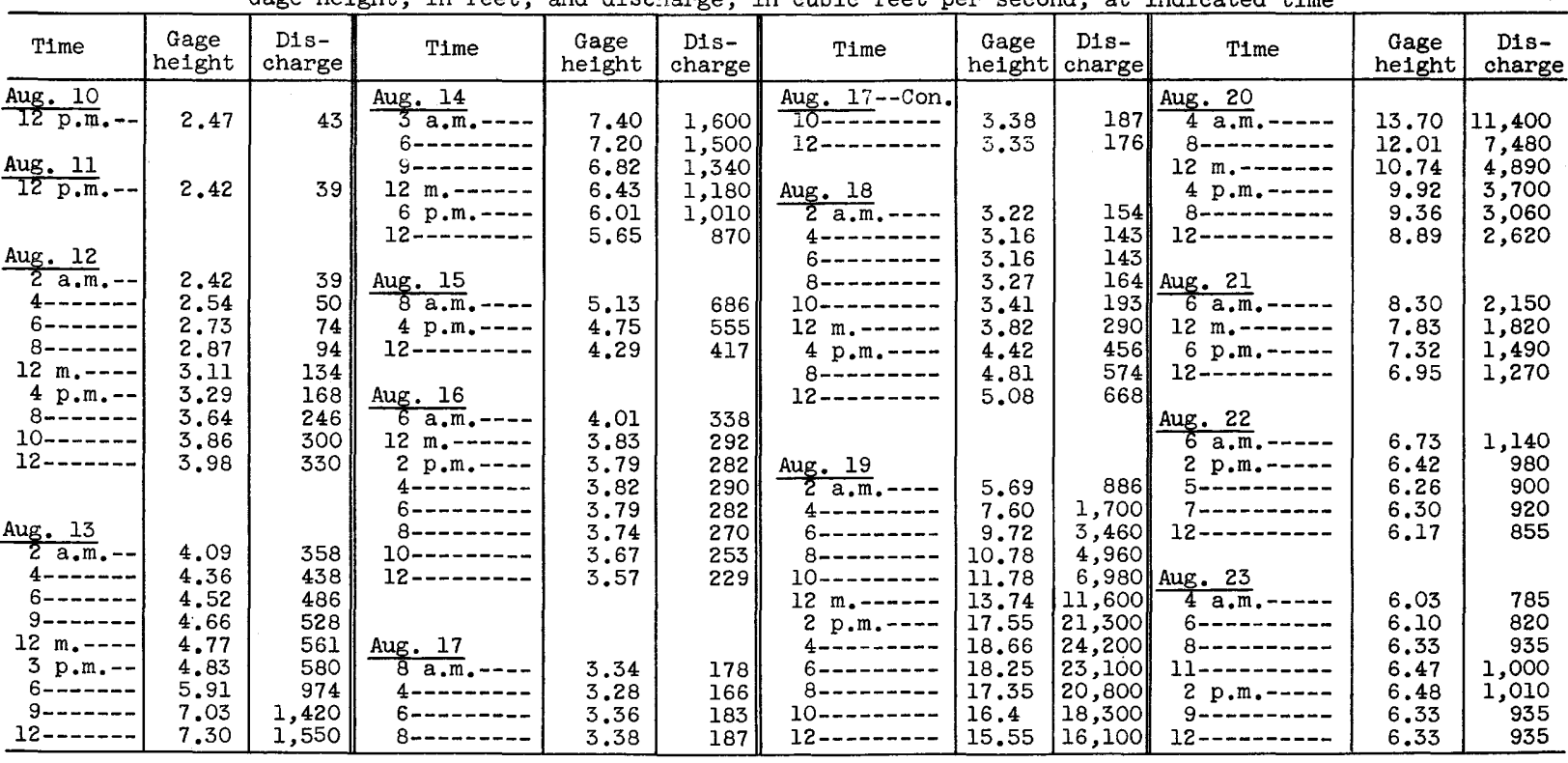

(30) zuinebaug River at Westville, Mass.

Location.--Lat $42^{\circ} 04^{\prime} 23^{\prime \prime}$, long $72^{\circ} 04^{\prime} 28^{\prime \prime}$, on right bank $350 \mathrm{ft}$ upstream from highway bridge, 0.45 mile downstream from of gage is $537.66 \mathrm{ft}$ above mean sea level, unadjusted (levels by Corps of Engineers).

Drainage area. --93.8 sq $\mathrm{mi}$

Gage-helght record. - -Water-stage recorder graph except for period 1 to 12 p.m. Aug. 19, when graph was drawn on basis of floodmarks. Stage graph adjusted for intake drawdown during period 8:30 a.m. Aug. 21 to 8 a.m. Aug. 22 and for intake lag during period Aug. 26-31.

Discharge record.--Stage-discharge relation defined by current-meter measurements below 1,300 cfs and extended to peak stage on basis of slope-area determination of peak flow.

Maxima --August 1955: Discharge, 17,500 cf s 6:30 p.m. Aug. 19 (gage helght, 16.11 ft, from floodmarks).

i 939 to July 1955: Discharge, 1,500 cf s Mar. 22 , 1948 (gage he1ght, $6.93 \mathrm{ft}$ ).

Flood in September 1938 reached a discharge of $8,400 \mathrm{cfs}$, by slope-area determination.

Remarks.--Flood flow affected by dam fallures and by reservolrs above station. Medium and low flows regulated by milis and reservolrs.

Mean discharge, in cubic feet per second, August 1955

\begin{tabular}{|c|c|c|c|c|c|c|c|c|c|c|c|}
\hline Day & Cfs & Day & Cfs & Day & Cfs & Day & Cfs & Day & Cfs & Day & Cfs \\
\hline $\begin{array}{l}1 \\
2 \\
3 \\
4 \\
5 \\
6\end{array}$ & $\begin{array}{l}34 \\
43 \\
45 \\
45 \\
41 \\
33\end{array}$ & $\begin{array}{r}7 \\
8 \\
9 \\
10 \\
11\end{array}$ & $\begin{array}{l}47 \\
65 \\
63 \\
54 \\
48\end{array}$ & $\begin{array}{l}12 \\
13 \\
14 \\
15 \\
16\end{array}$ & $\begin{array}{r}99 \\
252 \\
374 \\
433 \\
330\end{array}$ & $\begin{array}{l}17 \\
18 \\
19 \\
20 \\
21\end{array}$ & $\begin{array}{r}228 \\
605 \\
11,400 \\
8,830 \\
3,800\end{array}$ & $\begin{array}{l}22 \\
23 \\
24 \\
25 \\
26\end{array}$ & $\begin{array}{r}1,870 \\
1,230 \\
999 \\
774 \\
611\end{array}$ & $\begin{array}{l}27 \\
28 \\
29 \\
30 \\
31\end{array}$ & $\begin{array}{l}499 \\
436 \\
390 \\
365 \\
344\end{array}$ \\
\hline
\end{tabular}

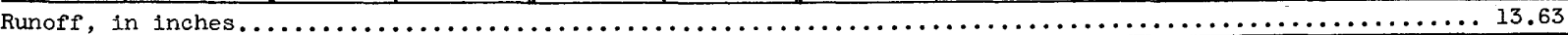


(30) Quinebaug River at Westville, Mass.--Continued

Gage helght, in feet, and discharge, in cublc feet per second, at indicated time

\begin{tabular}{|c|c|c|c|c|c|c|c|c|c|c|c|}
\hline Time & $\begin{array}{c}\text { Gage } \\
\text { helght }\end{array}$ & $\begin{array}{c}\text { Dis- } \\
\text { charge }\end{array}$ & Time & $\begin{array}{c}\text { Gage } \\
\text { height }\end{array}$ & $\begin{array}{c}\text { Dis- } \\
\text { charge }\end{array}$ & Time & $\begin{array}{c}\text { Gage } \\
\text { height }\end{array}$ & $\begin{array}{c}\text { D1s- } \\
\text { charge }\end{array}$ & Time & $\begin{array}{c}\text { Gage } \\
\text { height }\end{array}$ & $\begin{array}{c}\text { Dis- } \\
\text { charge }\end{array}$ \\
\hline$\frac{\text { Aug. } 17}{12}$ p. & 3.75 & 214 & 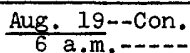 & 11.48 & 6,360 & $\frac{\text { Aug. } 20}{7 \mathrm{a} \mathrm{m}}-\mathrm{Con}$. & 13.60 & 10,200 & $\frac{\text { Aug. } 22}{4 \text { a.m. }}$ & 7.89 & 2,270 \\
\hline & & & & 12.69 & 8,410 & & 13.42 & 9,840 & & & \\
\hline ug. & & & $8-\cdots$ & 13.21 & 9,420 & $9-\cdots-$ & 13.26 & & $12 \mathrm{~m} .-$ & 7.28 & 1,820 \\
\hline 32 & 3.68 & 201 & $9-\ldots$ & 13.73 & 10,500 & $10-1-1$ & 13.14 & 9,2 & & 7.03 & 0 \\
\hline & 3.62 & 191 & $10 \ldots$ & 14.11 & 11,400 & $11-$. & 12.95 & 8,900 & & 6.80 & 1,500 \\
\hline $8--$ & 3.63 & 192 & $11-\ldots$ & 14.41 & 12,1 & $12 \mathrm{~m}$. & 12. & 8 , & $12-\cdots$ & 6.62 & 1,390 \\
\hline $10--$ & 3.64 & 194 & $12 \mathrm{~m} . \cdots$ & 14. & 12,8 & $2 \mathrm{p} . \mathrm{m}$ & 12. & & & & \\
\hline $11-$ & 3.68 & 201 & 1 p.m. & 14.94 & 13,600 & & 12.11 & & Aug. 23 & & \\
\hline $\begin{aligned} 12 \mathrm{~m} \\
1 \mathrm{p}\end{aligned}$ & $\begin{array}{l}3.80 \\
3.87\end{array}$ & 224 & $2-\cdots$ & 15.17 & 14, & $6---$ & 11.78 & 6,8 & $4 \mathrm{a} \cdot \mathrm{m}$ & 6.49 & $\begin{array}{l}1,310 \\
1,270\end{array}$ \\
\hline $2-7$ & $\begin{array}{l}3.87 \\
3.98\end{array}$ & $\begin{array}{l}268 \\
262\end{array}$ & 4 & $\begin{array}{l}15.42 \\
15.64\end{array}$ & $\begin{array}{l}15,100 \\
15,800\end{array}$ & 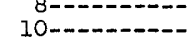 & $\begin{array}{l}11.44 \\
11.12\end{array}$ & $\begin{array}{l}6,300 \\
5,820\end{array}$ & $12 \mathrm{m.}=$ & $\begin{array}{l}6.42 \\
6.34\end{array}$ & $\begin{array}{l}1,270 \\
1,230\end{array}$ \\
\hline & 4.11 & 293 & $5-m-n$ & 15.87 & 16,600 & $12-\ldots$ & 10.84 & 5,420 & $4 \mathrm{p.m}$ & 6.25 & 1,180 \\
\hline & 4.38 & 365 & $6-\cdots$ & 16.08 & 17,400 & & & & & & 1,140 \\
\hline & 4.79 & 490 & $6: 30$ & 16.11 & 17,5 & Aug. & & & & 6.10 & 1,110 \\
\hline & 5.53 & 752 & & 16.0 & 17, & $2 a$ & 10.57 & 5,040 & & & \\
\hline & & 892 & 8----- & 15.95 & 16 , & $4-\ldots$ & 10.35 & 4,760 & Aug. 24 & & \\
\hline $8--$ & 6.65 & 1,280 & $9-\ldots$ & 15.70 & 16,000 & $6----$ & 10.11 & 4,460 & $6 \mathrm{a} . \mathrm{m}$. & 5.99 & 1,060 \\
\hline $9--$ & 7.22 & 1,620 & $10-$ & 15.50 & & $8---$ & 9.87 & & $12 \mathrm{~m} .-$ & & 1,000 \\
\hline 10 & 7.71 & 1,980 & $11-\ldots-$ & 15.2 & & $10--0-0$ & 9.67 & & $6 \mathrm{p.m}$. & & 942 \\
\hline & 8.23 & 2,410 & $12-$ & 15.01 & 13,800 & 12 m.-...- & 9.44 & 3,7 & $12-\cdots$ & 5.60 & 875 \\
\hline & & & Aug. 20 & & & $4---\cdots$ & 9.03 & & Aug. 25 & & \\
\hline ug. 1 & & & $1 \mathrm{a} \cdot \mathrm{m}_{0}$ & 14.77 & 13,100 & & 8.82 & 3,080 & $6 \mathrm{a} \cdot \mathrm{m}$ & 5.47 & 816 \\
\hline $1 a$. & 9.22 & 3,380 & $2-\cdots$ & 14.65 & 12,800 & $8 \ldots$ & 8.61 & & $12 \mathrm{~m}-\cdots$ & & 769 \\
\hline $2---$ & $\begin{array}{r}9.61 \\
10\end{array}$ & 3,820 & $3-\cdots$ & 14.31 & 11,900 & $10-10-$ & 8 & & 6 p.m.- & & 729 \\
\hline $4--$ & 10.46 & $\begin{array}{l}4,320 \\
4,890\end{array}$ & 4 & 14.05 & 11,200 & $12 \ldots \ldots$ & 8. & & & 5.16 & \\
\hline & 11.04 & 5,700 & $6--\cdots$ & 13.72 & $\begin{array}{l}10,500 \\
10,500\end{array}$ & & & & & & \\
\hline
\end{tabular}

(39) French R1ver at Webster, Mass.

Location.--Lat $42^{\circ} 03^{\prime} 03^{\prime \prime}$, long $71^{\circ} 53^{\prime} 08^{\prime \prime}$, on right bank $50 \mathrm{ft}$ upstream from Pleasant Street Bridge at Webster, Worcester County, and 1.1 miles upstream from Potash Brook. Datum of gage is $406.74 \mathrm{ft}$ above mean sea level, datum of 1929

Drainage area. $--85.3 \mathrm{sq} \mathrm{m} 1$

Gage-helght record.--Water-stage recorder graph except for period 1:15 p.m. Aug. 19 to 4:30 p.m. Aug. 22 Gage heights for period $1: 15 \mathrm{p.m}$. to $6 \mathrm{p.m}$. Aug. 19 are from graph based on floodmarks and readings from reference points and for period $4 \mathrm{pm}$. Aug. 21 to noon Aug. 22 from graph based on engineer's tape-gage reading.

D1scharge record.--Stage-discharge relation defined by current-meter measurements below 2 , 400 cfs and extended to peak stage on basis of computation of peak flow over dam. Discharge for period 8 p.m. Aug. 19 to noon Aug. 21 estimated on basis of records for Quinebaug River at Westville and Littie River at Buffumvilie. Backwater from debris at times.

Maxima.--August 1955: D1scharge, 14,400 cfs 6 p.m. Aug. 19 (gage helght, 26.05 ft, from floodmarks). 1948 to July 1955: Discharge, 2,320 cf's Sept. 12, 1954 (gage height, $11.64 \mathrm{ft}$ ).

Flood of Mar. 19, 1936, reached a discharge of 4,700 cfs, by computation of flow over dam.

Remarks.--Flood flow affected by dam fallures and by reservoirs above station. Low and medium flows regulated by Lake Chaubunagungamaug (estimated usable capacity, 207,000,000 cu ft) and other reservolrs above station.

\begin{tabular}{|c|c|c|c|c|c|c|c|c|c|c|c|}
\hline Day & Cfs & Day & Cf's & Day & $\mathrm{Cfs}$ & Day & Cfs & Day & Cfs & Day & $\mathrm{Cfs}$ \\
\hline $\begin{array}{l}1 \\
2 \\
3 \\
4 \\
5 \\
6\end{array}$ & $\begin{array}{c}39 \\
42 \\
29 \\
24 \\
44 \\
9.6\end{array}$ & $\begin{array}{r}7 \\
8 \\
9 \\
10 \\
11\end{array}$ & $\begin{array}{l}14 \\
50 \\
44 \\
49 \\
36\end{array}$ & $\begin{array}{l}12 \\
13 \\
14 \\
15 \\
16\end{array}$ & $\begin{array}{r}43 \\
48 \\
96 \\
97 \\
131\end{array}$ & $\begin{array}{l}17 \\
18 \\
19 \\
20 \\
21\end{array}$ & $\begin{array}{r}125 \\
222 \\
6,170 \\
5,640 \\
2,180\end{array}$ & $\begin{array}{l}22 \\
23 \\
24 \\
25 \\
26\end{array}$ & $\begin{array}{r}1,290 \\
937 \\
782 \\
730 \\
720\end{array}$ & $\begin{array}{l}27 \\
28 \\
29 \\
30 \\
31\end{array}$ & $\begin{array}{l}618 \\
540 \\
464 \\
436 \\
422\end{array}$ \\
\hline
\end{tabular}

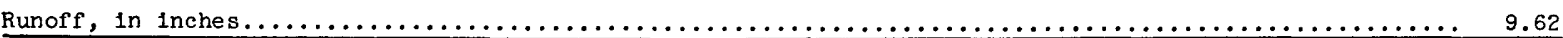

\begin{tabular}{|c|c|c|c|c|c|c|c|c|c|c|c|}
\hline Time & $\begin{array}{c}\text { Gage } \\
\text { height }\end{array}$ & $\begin{array}{c}\text { Dis- } \\
\text { charge }\end{array}$ & Time & $\begin{array}{c}\text { Gage } \\
\text { he1ght }\end{array}$ & $\begin{array}{c}\text { Dis- } \\
\text { charge }\end{array}$ & Time & $\begin{array}{c}\text { Gage } \\
\text { height }\end{array}$ & $\begin{array}{l}\text { Dis- } \\
\text { charge }\end{array}$ & Time & $\begin{array}{c}\text { Gage } \\
\text { height }\end{array}$ & $\begin{array}{l}\text { Dis- } \\
\text { charge }\end{array}$ \\
\hline 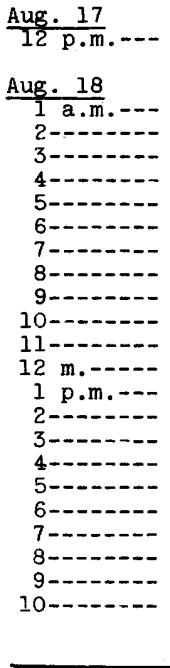 & $\begin{array}{l}4.94 \\
4.98 \\
5.01 \\
5.03 \\
5.05 \\
5.34 \\
5.63 \\
5.55 \\
5.48 \\
5.45 \\
5.63 \\
5.52 \\
5.72 \\
5.63 \\
5.52 \\
5.50 \\
5.73 \\
5.69 \\
5.80 \\
5.86 \\
5.94 \\
6.01\end{array}$ & $\begin{array}{r}99 \\
104 \\
109 \\
112 \\
115 \\
167 \\
239 \\
218 \\
199 \\
192 \\
239 \\
209 \\
263 \\
239 \\
236 \\
204 \\
266 \\
255 \\
285 \\
302 \\
324 \\
344\end{array}$ & 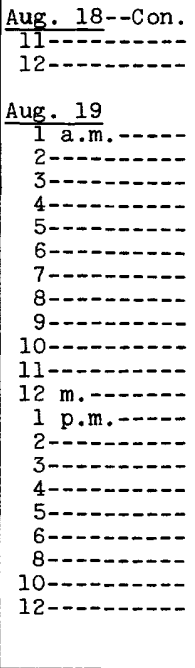 & $\begin{array}{r}6.30 \\
6.56 \\
7.15 \\
7.66 \\
8.87 \\
9.41 \\
10.09 \\
10.75 \\
11.57 \\
12.57 \\
14.28 \\
16.39 \\
19.22 \\
20.00 \\
20.85 \\
21.77 \\
24.00 \\
26.05\end{array}$ & $\begin{array}{r}369 \\
397 \\
\\
425 \\
498 \\
663 \\
813 \\
1,210 \\
1,400 \\
1,670 \\
1,930 \\
2,280 \\
2,780 \\
3,670 \\
4,970 \\
7,200 \\
7,900 \\
8,660 \\
9,570 \\
11,900 \\
14,400 \\
13,000 \\
11,400 \\
10,000\end{array}$ & 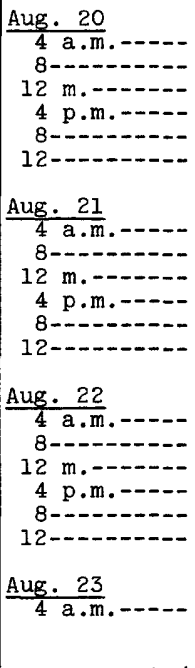 & $\begin{array}{r}10.59 \\
10.34 \\
10.12 \\
9.93 \\
9.72 \\
9.54\end{array}$ & $\begin{array}{l}7,900 \\
6,300 \\
5,200 \\
4,300 \\
3,600 \\
3,100 \\
\\
2,700 \\
2,350 \\
2,100 \\
1,860 \\
1,710 \\
1,580\end{array}$ & 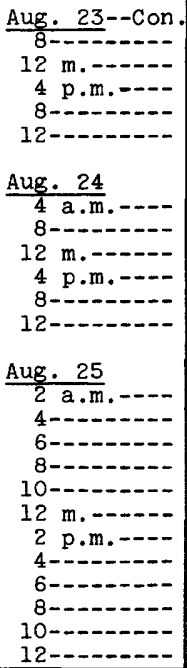 & $\begin{array}{l}8.45 \\
8.40 \\
8.38 \\
8.40 \\
8.52 \\
8.69 \\
8.86 \\
8.96 \\
9.00 \\
8.99 \\
8.94 \\
8.89\end{array}$ & $\begin{array}{l}649 \\
635 \\
629 \\
635 \\
671 \\
726 \\
786 \\
819 \\
834 \\
831 \\
813 \\
795\end{array}$ \\
\hline
\end{tabular}


(45) Quinebaug River at Jewett City, Conn.

Location.--Lat $41^{\circ} 35^{\prime} 52^{\prime \prime}$, long $71^{\circ} 59^{\prime} 05^{\prime \prime}$, on left bank in rear of school house on Slater Avenue at Jewett City,

New London County, $570 \mathrm{ft}$ downstream from outlet of canal from Fisk M11ls, Inc. at mouth of Pachaug R1ver, 1,000

$\mathrm{ft}$ downstream from rall road bridge, and at mile 6.1. Datum of gage $1 \mathrm{~s} 63.07 \mathrm{ft}$ dibove mean sea level, datum of 1929 . Drainage area. --711 sq $\mathrm{ml}$.

Gage-height record.--Water-stage recorder graph except for period 2 p.m. Aug. 19 to 3 p.m. Aug. 22 . Graph for this period drawn on basis of floodmark and time of peak.

Discharge record.--Stage-discharge relation defined by current-meter measurements below 11,000 cfs, and by computation

of flow over three nearby dams at gage helghts $21.7,22,5$, and $24.0 \mathrm{ft}$, and at dam 6 miles downstream at gage height $29.0 \mathrm{ft}$. Shifting-control method used at times.

Maxima.--August 1955: Discharge, $40,700 \mathrm{cfs} 5$ to $6 \mathrm{p.m}$. Aug. 20 (gage height, $29.0 \mathrm{ft}$, from floodmarks).

T918 to July 1955: Discharge, 29,200 cf s Mar. 19, 1936 (gage height, $24.0 \mathrm{ft}$, from floodmarks).

Mean discharge, in cublc feet per second, August 1955

\begin{tabular}{c|r|r|r||r|r|r|r||r|r|r|r}
\hline Day & Cfs & Day & Cfs & Day & Cfs & Day & Cfs & Day & Cfs & Day & Cfs \\
\hline 1 & 337 & 7 & 106 & 12 & 360 & 17 & 998 & 22 & 11,200 & 27 & 3,110 \\
2 & 291 & 8 & 348 & 13 & 1,210 & 18 & 1,060 & 23 & 6,770 & 28 & 2,750 \\
3 & 272 & 9 & 289 & 14 & 1,610 & 19 & 7,020 & 24 & 5,800 & 29 & 2,390 \\
4 & 267 & 10 & 299 & 15 & 1,600 & 20 & 35,300 & 25 & 4,680 & 30 & 2,070 \\
5 & 126 & 11 & 375 & 16 & 1,260 & 21 & 25,100 & 26 & 3,750 & 31 & 1,940 \\
6 & 42 & & & & & & & & & & \\
\hline
\end{tabular}

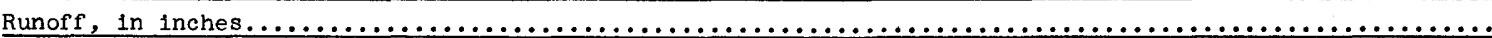

6.42

Gage height, in feet, and discharge, in cublc feet per second, at indicated time

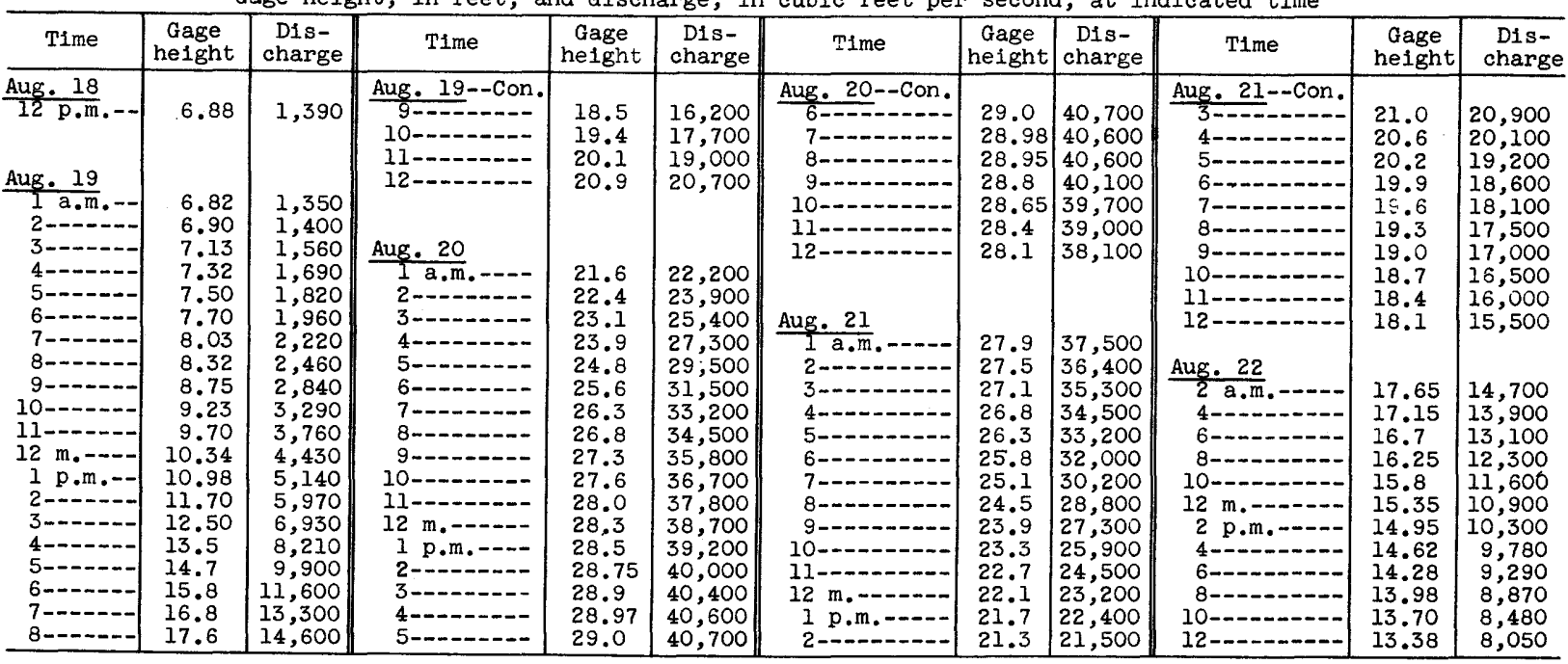


(47) Connecticut River at Montague City, Mass.

Location.--Lat $42^{\circ} 34^{\prime} 48^{\prime \prime}$, long $72^{\circ} 34^{\prime} 30^{\prime \prime}$, on left bank 75 ft downstream from New York, New Haven \& Hartford Rälroad bridge at Montague City, Franklin County, and 1 , 0 ju ft downstream from Deerfield River. Datum of gage is $99.87 \mathrm{ft}$ above mean sea level, datum of 1929 .

Drainage area. $--7,865 \mathrm{sq} \mathrm{mi}$.

Gage-height record.--Water-stage recorder graph.

Discharge record.-Stage-discharge relation defined by current-meter measurements.

Maxima.-August i955: Discharge, $42,800 \mathrm{cfs} 1$ to $1: 30 \mathrm{p.m}$. Aug. 14 (gage height, $19.75 \mathrm{ft}$ ).

i904 to July 1955: Discharge, 236 , 000 cfs Mar. 19, i936 (gage helght, 49.2 ft, from floodmarks), from rating curve extended above $160,000 \mathrm{cfs}$.

Remarks.--Flow regulated by powerplants and by First Connecticut and Second Connecticut Lakes, Lake Franc1s, Comerford Station Pond, and other reservolrs (combined usabie capacity, about $27 \frac{1}{2}$ billion cu ft).

\begin{tabular}{|c|c|c|c|c|c|c|c|c|c|c|c|}
\hline Day & $\mathrm{Cfs}$ & Day & $\mathrm{Cfs}$ & Day & $\mathrm{Cfs}$ & Day & $\mathrm{Cfs}$ & Day & $\mathrm{Cfs}$ & Day & $\mathrm{Cfs}$ \\
\hline $\begin{array}{l}1 \\
2 \\
3 \\
4 \\
5 \\
6\end{array}$ & $\begin{array}{r}2,780 \\
3,420 \\
2,000 \\
1,990 \\
1,940 \\
601\end{array}$ & $\begin{array}{r}7 \\
8 \\
9 \\
10 \\
11\end{array}$ & $\begin{array}{r}581 \\
1,010 \\
2,460 \\
2,980 \\
6,000\end{array}$ & $\begin{array}{l}12 \\
13 \\
14 \\
15 \\
16\end{array}$ & $\begin{array}{r}9,720 \\
16,800 \\
39,400 \\
33,300 \\
18,800\end{array}$ & $\begin{array}{l}17 \\
18 \\
19 \\
20 \\
21\end{array}$ & $\begin{array}{l}17,600 \\
20,100 \\
33,400 \\
26,000 \\
13,500\end{array}$ & $\begin{array}{l}22 \\
23 \\
24 \\
25 \\
26\end{array}$ & $\begin{array}{r}10,800 \\
9,610 \\
10,800 \\
11,500 \\
10,400\end{array}$ & $\begin{array}{l}27 \\
28 \\
29 \\
30 \\
31\end{array}$ & $\begin{array}{l}5,920 \\
3,640 \\
7,300 \\
7,340 \\
7,540\end{array}$ \\
\hline
\end{tabular}

Gage height, in feet, and discharge, in cublc feet per second, at indicated time

\begin{tabular}{|c|c|c|c|c|c|c|c|c|c|c|c|}
\hline $\mathrm{T} 1$ & & $\begin{array}{l}\text { Dis- } \\
\text { charge }\end{array}$ & Time & & $\begin{array}{l}\text { Dis- } \\
\text { charge }\end{array}$ & Time & & $\begin{array}{c}\text { Dis- } \\
\text { charge }\end{array}$ & Time & & $\begin{array}{l}\text { Dis- } \\
\text { charge }\end{array}$ \\
\hline 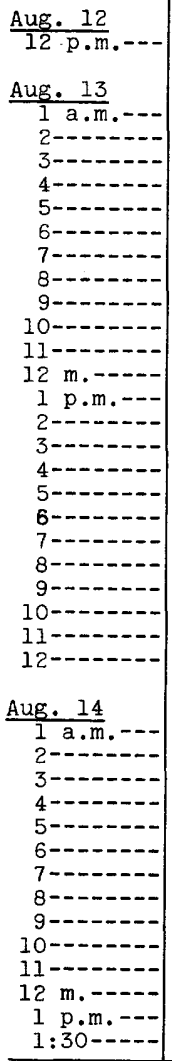 & $\begin{array}{r}11.63 \\
11.04 \\
10.53 \\
9.89 \\
9.40 \\
9.52 \\
10.32 \\
10.95 \\
11.47 \\
11.47 \\
11.13 \\
11.85 \\
12.34 \\
12.69 \\
12.28 \\
12.35 \\
13.03 \\
13.54 \\
15.16 \\
15.77 \\
15.87 \\
16.50 \\
17.18 \\
17.45\end{array}$ & $\begin{array}{r}13,700 \\
12,000 \\
10,600 \\
9,010 \\
7,930 \\
8,180 \\
10,000 \\
11,800 \\
13,200 \\
13,200 \\
12,300 \\
14,400 \\
15,800 \\
16,900 \\
15,600 \\
15,800 \\
17,900 \\
19,500 \\
25,000 \\
27,200 \\
27,500 \\
29,900 \\
32,500 \\
33,500\end{array}$ & 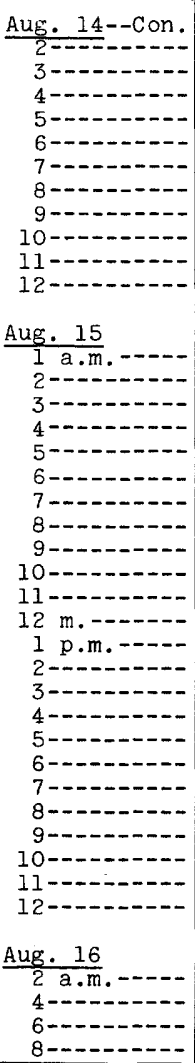 & $\begin{array}{l}19.39 \\
19.41 \\
19.41 \\
19.38 \\
19.18 \\
19.16 \\
19.25 \\
19.27 \\
19.17 \\
18.89 \\
18.64 \\
18.40 \\
17.39 \\
16.97 \\
17.05 \\
16.73 \\
16.18 \\
15.17 \\
14.54 \\
14.19 \\
13.84 \\
13.76 \\
13.67 \\
13.60\end{array}$ & $\begin{array}{l}41,300 \\
41,300 \\
41,300 \\
41,200 \\
40,400 \\
40,300 \\
40,700 \\
40,800 \\
40,400 \\
39,300 \\
38,300 \\
37,300 \\
33,300 \\
31,700 \\
32,000 \\
30,800 \\
28,700 \\
25,000 \\
22,800 \\
21,600 \\
20,500 \\
20,200 \\
19,900 \\
19,700 \\
\end{array}$ & 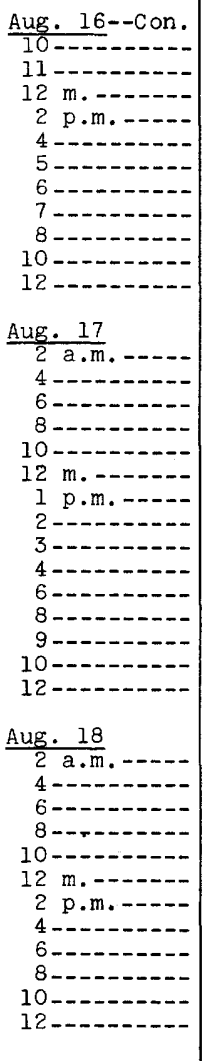 & $\begin{array}{l}13.63 \\
13.72 \\
13.65 \\
13.21 \\
13.07 \\
13.07 \\
13.25 \\
13.28 \\
13.22 \\
13.05 \\
13.08\end{array}$ & $\begin{array}{l}19,800 \\
20,100 \\
19,900 \\
18,500 \\
18,000 \\
18,000 \\
18,600 \\
18,700 \\
18,500 \\
18,000 \\
18,100\end{array}$ & 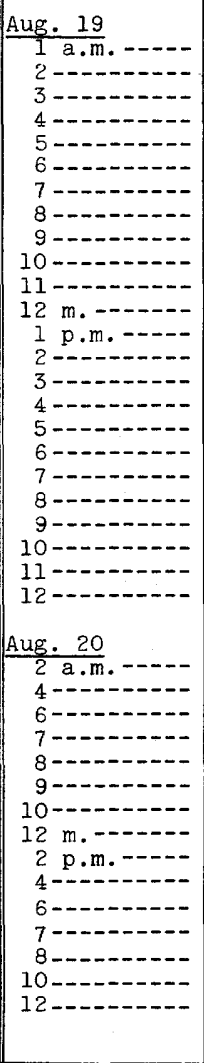 & & $\begin{array}{l}23,500 \\
23,400 \\
23,500 \\
23,800 \\
24,300 \\
25,200 \\
26,400 \\
31,600 \\
34,700 \\
36,000 \\
37,600 \\
38,300 \\
39,200 \\
40,500 \\
41,100 \\
40,800 \\
40,300 \\
39,500 \\
38,500 \\
37,600 \\
36,700 \\
36,000 \\
35,100 \\
33,200\end{array}$ \\
\hline
\end{tabular}


(55) Ware River at Gibbs Crossing, Mass.

Location.--Lat $42^{\circ} 14^{\prime} 07^{\prime \prime}$, long $72^{\circ} 16^{\circ} 45^{\prime \prime}$, on right bank half a mile upstream from Gibbs Crossing, Hampshire County, $1.8 \mathrm{miles}$ upstream from Beaver Brook, and $2 \frac{1}{2} \mathrm{miles}$ southwest of Ware. Datum of gage is $379.79 \mathrm{ft}$ above mean sea level, datum of 1929 .

Drainage area.--199 $\mathrm{sq} \mathrm{mi}$.

Gage-height record.--Water-stage recorder graph.

Discharge record.--Stage-discharge relation defined by current-meter measurements below 4,600 cfs and extended to peak stage on basis of contracted-opening determination at $12.83 \mathrm{ft}$ and slope-area determination at $18.2 \mathrm{ft}$. Backwater from grass at times.

Maxima.--August 1955: Discharge, $12,200 \mathrm{cfs} 10 \mathrm{a} . \mathrm{m}$. Aug. 19 (gage helght, $12.83 \mathrm{ft}$ ).

1912 to July 1955: Discharge, $22,700 \mathrm{cf}$ s Sept. 21, 1938 (gage height, $18.2 \mathrm{ft}$, from floodmarks), from slopearea determination.

Remarks.--Flood flow affected by diversion from $97 \mathrm{sq} \mathrm{mi}$ for supply of Boston metropolitan district. Regulation at low and medium flows by mills above station.

Mean discharge, in cubic feet per second, August 1955

\begin{tabular}{r|r||r|r|r|r||r|r||r|r|r|r|r}
\hline Day & Cfs & Day & Cfs & Day & Cfs & Day & Cfs & Day & Cfs & Day & Cfs \\
\hline 1 & 78 & 7 & 28 & 12 & 87 & 17 & 199 & 22 & 1,000 & 27 & 685 \\
2 & 57 & 8 & 75 & 13 & 166 & 18 & 1,550 & 23 & 1,260 & 28 & 625 \\
3 & 22 & 9 & 27 & 14 & 565 & 19 & 8,880 & 24 & 1,160 & 29 & 553 \\
4 & 21 & 10 & 61 & 15 & 553 & 20 & 5,060 & 25 & 812 & 30 & 436 \\
5 & 21 & 11 & 31 & 16 & 370 & 21 & 1,930 & 26 & 842 & 31 \\
6 & 21 & & & & & & & \\
\hline
\end{tabular}

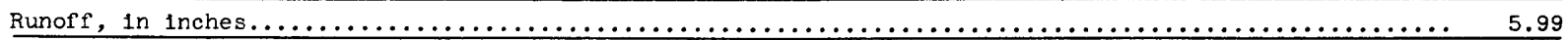

Gage height, in feet, and discharge, in cubic feet per second, at indicated time

\begin{tabular}{|c|c|c|c|c|c|c|c|c|c|c|c|}
\hline Time & $\begin{array}{c}\text { Gare } \\
\text { he1ght }\end{array}$ & $\begin{array}{l}\text { Dis- } \\
\text { charge }\end{array}$ & Ti & $\begin{array}{l}\text { Gage } \\
\text { he1ght }\end{array}$ & $\begin{array}{l}\text { Dis- } \\
\text { charge }\end{array}$ & Time & $\begin{array}{l}\text { Gage } \\
\text { he1ght }\end{array}$ & $\begin{array}{c}\text { D1s- } \\
\text { charge }\end{array}$ & Time & $\begin{array}{l}\text { Gage } \\
\text { height }\end{array}$ & $\begin{array}{l}\text { Dis- } \\
\text { eharge }\end{array}$ \\
\hline$\frac{\text { Aug. } 17}{12 \mathrm{p} \cdot \mathrm{m}}$ & 2.52 & 179 & Aug. 18--Con. & 6.33 & 3,270 & Aug. 19--Con. & 10.29 & 8,140 & Aug. $22--$ Con. & & 1,0 \\
\hline Aug. 18 & & & Aug. 19 & & & Aug. 20 & & & $12 \mathrm{m.}-$ & & 1,00 \\
\hline $1 \mathrm{a.m}$. & 2.51 & 175 & $1 \mathrm{a} . \mathrm{m}_{0}-$ & 6.35 & 3,290 & a a.m. & 9.82 & 7,480 & $6 \mathrm{p.m}$. & 3.77 & 9 \\
\hline $3-$ & $\begin{array}{l}2.51 \\
2.50\end{array}$ & $\begin{array}{l}175 \\
172\end{array}$ & $\begin{array}{l}2-\cdots \\
3-\cdots\end{array}$ & & & $\begin{array}{l}4-- \\
6--\end{array}$ & & & $\begin{array}{c}8-\cdots- \\
10 \cdots\end{array}$ & & $\begin{array}{l}97 . \\
9 .\end{array}$ \\
\hline $4--$ & 2.50 & 172 & $4-\cdots$ & 7. & 4,160 & $8 \cdots$ & 8.51 & 5,730 & $12-$ & 3.77 & \\
\hline $5---$ & 2.49 & 168 & $5---$ & 8. & 5,5 & $10--$ & & 5,250 & & & \\
\hline $6---$ & 2.49 & 168 & $6---$ & 9. & 7,3 & $12 \mathrm{m.--}$ & 7.7 & 4,830 & Aug. 23 & & \\
\hline $7-\cdots$ & 2.50 & 172 & $7-\ldots-\infty$ & 10. & $9, i$ & $2 \mathrm{p.m}$. & & 4,390 & $6 \mathrm{a} \cdot \mathrm{m}$. & 4.10 & 1,2 \\
\hline $8---5$ & 2.54 & 187 & $8-\cdots$ & 12. & 10,8 & & & 4,110 & & & 3 \\
\hline $9-\cdots-1$ & 2.57 & 199 & & & & & & & $10-\cdots$ & & \\
\hline $10-1 . . .+2$ & 2.58 & 203 & $10-00-1$ & 3 & & $8---$ & & & $12 \mathrm{m.-}$ & & 1,2 \\
\hline $11---0$ & 2.66 & 23 & $11-0--0$ & 6 & & $10-\ldots$ & & 3,140 & $6 \mathrm{pm}$ & & 1,3 \\
\hline $12 \mathrm{~m}$ & 2.96 & 385 & $12 \mathrm{m.}$. & 12. & & $12-\ldots$ & 5.94 & 2,880 & $12=--$ & & 1,3 \\
\hline 1 p.m. & 3.66 & 828 & I p.m. & 12.42 & & & & & & & \\
\hline & 4.: & 1,950 & $2-\cdots$ & 27 & & Aug. 21 & & & Aug. & & \\
\hline $3--$ & 5.76 & 2,7 & $3---$ & 12.14 & 11,0 & $4 \overline{\mathrm{a}} \mathrm{m}$, & 5.45 & 2,440 & $2 \mathrm{a} \cdot \mathrm{m}$. & .24 & 1,35 \\
\hline $4---$ & 6.25 & & & 12.03 & & $8 \cdots-$ & & & & 13 & 2 \\
\hline & & & $5-\cdots$ & & & $12 \mathrm{~m} . \cdots$ & & & & & 23 \\
\hline $6---$ & 6.5 & & $6---\cdots$ & 11.73 & 10 , & 4 p.m. & & $\$ 50$ & $8--$ & & 1,25 \\
\hline$=--$ & 6.64 & & $7-\cdots$ & 11.53 & 10,000 & $8 \cdots$ & 4.34 & 1,440 & $12 \mathrm{~m} .--$ & & 1,15 \\
\hline $8 \ldots \ldots$ & 6.64 & & & 11.29 & & $12--$ & 4.12 & 1,240 & 6 p.m.-- & & 1,04 \\
\hline $9-->>$ & 6.57 & & & 11.04 & & & & & & 3.75 & \\
\hline $10--$ & 6. & & $10 \cdots$ & 10. & & Aug. 22 & & & & & \\
\hline-- & .39 & & $11--\cdots$ & 10.53 & 8,500 & $6 \mathrm{a} . \mathrm{m} .-\cdots$ & 3.79 & 962 & & & \\
\hline
\end{tabular}

(61) 2uaboag River at West Brimfield, Mass.

Location.--Lat $42^{\circ} 10^{\prime} 31^{\prime \prime}$, long $72^{\circ} 15^{\prime} 46^{\prime \prime}$, on left bank $15 \mathrm{ft}$ upstream from site of former highway bridge at west Brimfield, Hampden County, 0.4 mile upstream from Blodgett Mill Brook, and $3 \frac{1}{2}$ miles northeast of Palmer. Datum of gage is $377.36 \mathrm{ft}$ above mean sea level, datum of 1929.

Drainage area. $-151 \mathrm{sq} \mathrm{mi}$.

Gage-he1ght record.--Water-stage recorder graph except for 8:15 to 9:15 a.m. Aug. 19; floodmark used to complete graph.

D1scharge record.--Stage-discharge relation defined by current-meter measurements below 2,700 cfs and extended to peak stage on basis of slope-area determinations at gage heights 11.8 and $14.79 \mathrm{ft}$.

Maxima.--August 1955: Discharge, 12,800 cf s 8:30 a.m. Aug. 19 (gage height, 14.79 ft, from floodmark in gage house). 1909 to July 1955: Discharge, $8,470 \mathrm{cfs}$ Sept. 2i, 1938 (gage height, $11.8 \mathrm{ft}$, from floodmarks), from slope-

Remarks.--Flood flow affected by natural storage. Slight diurnal regulation at low flow by mill above station.

\begin{tabular}{|c|c|c|c|c|c|c|c|c|c|c|c|}
\hline Day & Cfs & Day & $\mathrm{Cfs}$ & Day & Cfs & Day & Cfs & Day & Cfs & Day & Cfs \\
\hline $\begin{array}{l}1 \\
2 \\
3 \\
4 \\
5 \\
6\end{array}$ & $\begin{array}{l}41 \\
38 \\
38 \\
35 \\
35 \\
32\end{array}$ & $\begin{array}{r}7 \\
8 \\
9 \\
10 \\
11\end{array}$ & $\begin{array}{l}42 \\
65 \\
48 \\
45 \\
46\end{array}$ & $\begin{array}{l}12 \\
13 \\
14 \\
15 \\
16\end{array}$ & $\begin{array}{r}84 \\
338 \\
435 \\
352 \\
302\end{array}$ & $\begin{array}{l}17 \\
18 \\
19 \\
20 \\
21\end{array}$ & $\begin{array}{r}274 \\
1,220 \\
7,590 \\
6,370 \\
5,360\end{array}$ & $\begin{array}{l}22 \\
23 \\
24 \\
25 \\
26\end{array}$ & $\begin{array}{l}4,270 \\
3,640 \\
3,020 \\
2,500 \\
2,090\end{array}$ & $\begin{array}{l}27 \\
28 \\
29 \\
30 \\
31\end{array}$ & $\begin{array}{r}1,730 \\
1,440 \\
1,220 \\
1,040 \\
910\end{array}$ \\
\hline
\end{tabular}


FLOOD DATA

(61) Quaboag River at West Brimf'ield, Mass.--Continued

Gage helght, in feet, and discharge, in cublc feet per second, at indicated time

\begin{tabular}{|c|c|c|c|c|c|c|c|c|c|c|c|}
\hline Time & $\begin{array}{c}\text { Gage } \\
\text { he1ght }\end{array}$ & $\begin{array}{l}\text { Dis- } \\
\text { charge }\end{array}$ & Time & $\begin{array}{c}\text { Gage } \\
\text { he1ght }\end{array}$ & $\begin{array}{l}\text { Dis- } \\
\text { charge }\end{array}$ & Time & $\begin{array}{c}\text { Gage } \\
\text { he1ght }\end{array}$ & $\begin{array}{c}\text { Dis- } \\
\text { charge }\end{array}$ & Time & $\begin{array}{c}\text { Gage } \\
\text { he1ght }\end{array}$ & $\begin{array}{c}\text { Dis- } \\
\text { charge }\end{array}$ \\
\hline$\frac{\text { Aug. } 17}{12 \text { p.m. }}$ & 3.38 & 265 & $\frac{\text { Aug. } 19}{1 . \mathrm{a} \cdot \mathrm{m}}$ & $\begin{array}{l}5.99 \\
6.36 \\
6.90\end{array}$ & $\begin{array}{l}2,130 \\
2,460 \\
2,950\end{array}$ & Aug. $19--C o n$ & $\begin{array}{l}10.46 \\
10.42\end{array}$ & $\begin{array}{l}6,800 \\
6,750\end{array}$ & $\frac{\text { Aug. } 20--C o}{8-}$ & $\begin{array}{l}9.91 \\
9.85 \\
9.82\end{array}$ & $\begin{array}{l}6,140 \\
6,070 \\
6,030\end{array}$ \\
\hline Aug. 18 & & & & 7.70 & 3,730 & Aug. 20 & & & $11-\ldots$ & 9.78 & 5,990 \\
\hline $\begin{array}{l}6 \mathrm{a} . \mathrm{m} \\
7 \mathrm{-} .\end{array}$ & $\begin{array}{l}3.38 \\
3.39\end{array}$ & $\begin{array}{l}265 \\
270\end{array}$ & $\begin{array}{l}5-- \\
6--\end{array}$ & $\begin{array}{r}9.05 \\
10.77\end{array}$ & $\begin{array}{l}5,160 \\
7,170\end{array}$ & $\begin{array}{l}1 \mathrm{a} \cdot \mathrm{m} \\
2-\end{array}$ & $\begin{array}{l}10.40 \\
10.38\end{array}$ & $\begin{array}{l}6,730 \\
6,710\end{array}$ & $12 \ldots$ & 9.74 & 5,940 \\
\hline $8---$ & 3.41 & 279 & $7 \ldots$ & 13.96 & 11,6 & & 10.39 & 6,720 & Aug. 21 & & \\
\hline $9-\cdots$ & 3.44 & 293 & $8----$ & 14.27 & 12,0 & $4-\cdots$ & 10.29 & 6,600 & $\frac{1}{6} \mathrm{a} \cdot \mathrm{m}$ & 9.49 & 5,640 \\
\hline $10 \ldots$ & 3.46 & 302 & $8: 30-\ldots$ & 14.79 & 12,8 & $5-\ldots$ & 10.28 & 6,590 & $12 \mathrm{~m} .-\cdots$ & & 5,360 \\
\hline $11-\ldots$ & 3.53 & 336 & $9-\cdots-1-$ & 14.51 & 12,4 & $6-\cdots$ & 10.27 & 6,570 & 6 p.m. & 8.97 & 5,070 \\
\hline $12 \mathrm{m.-}$ & 3.66 & 403 & $10 \ldots$ & 13. & 11 , & $7-\ldots$ & 10.15 & 6,430 & & 8.71 & 4,780 \\
\hline $1 \mathrm{p} . \mathrm{n}$ & 4.03 & .603 & $11-1$ & 13.48 & & & & & & & \\
\hline & 6.12 & 2,250 & $12 \mathrm{~m} . \cdots$ & 13.14 & 10,300 & $9 \ldots \ldots$ & 10.15 & 6,430 & Aug. 22 & & \\
\hline $3--$ & 7.35 & 3,380 & $1 \mathrm{p.m}$. & 12.70 & 9,710 & $10---$ & 10.14 & 6,420 & $\mathrm{~b} \mathrm{a} \cdot \mathrm{m}$ & 8.45 & 4,500 \\
\hline $4--$ & 6.63 & & $2-\cdots$ & 12.29 & 9,1 & $11 \ldots$ & 10.13 & 6,410 & $12 \mathrm{m.--}$ & 8.22 & 4,250 \\
\hline $5-\cdots$ & 6.39 & & $3 \ldots$ & 11.91 & & $12 \mathrm{~m}$. & 10.11 & & 6 p.m. & 7.99 & 4,020 \\
\hline $6-\ldots$ & 6.39 & 2,490 & 4 & 11.62 & 8,240 & l p.m. & 10.10 & 6,370 & $12 \ldots$ & 7.79 & 3,820 \\
\hline $7--$ & 6.32 & & $5-\cdots$ & 11.39 & 7, & & 10.09 & & & & \\
\hline & 6.16 & & $6-\cdots$ & 11.05 & & & 10.06 & & Aug. 23 & & \\
\hline & & & $7-\cdots$ & & & & 10.04 & 6,300 & $2 \mathrm{a} \cdot \mathrm{m}$. & 7.88 & 3,910 \\
\hline $10 \cdots$ & 5.85 & & $8 \cdots-\cdots$ & 10.51 & & $5 \ldots$ & 10.01 & & & 7.87 & 3,900 \\
\hline $11-\ldots$ & 5.79 & & $9-\ldots$ & 10.49 & 6,840 & & 9.97 & & $12 \mathrm{m.c}$ & 7.61 & 3,640 \\
\hline $12-\ldots$ & 5.79 & 1,950 & $10 \ldots$ & 10.49 & 6,840 & 7 & 9.94 & 6,180 & 12 p.m. & 7.28 & 3,310 \\
\hline
\end{tabular}

(68) Chicopee River at Indian Orchard, Mass.

Location - Iat $42^{\circ} 09^{\prime} 38^{\prime \prime}$, long $72^{\circ} 30^{\prime} 52^{\prime \prime}$, on left bank 1,000 ft downstream from West Street Bridge at Indian Orchard, Hampden County, and 1.1 miles upstream from Fuller Brook. Altitude of gage is 125 ft (from topographic map).

Drainage area. $--688 \mathrm{sq} \mathrm{ml}$.

Gage-helsht record.--itater-stage recorder graph.

Discharge record.--Stage-discharge relation defined by current-meter measurements below 22,000 cfs and extended to peak stage on basis of computation of peak flow over dam. Backwater from grass at times.

Maxima.--August 1955: Discharge, 40,500 cfs 4 p.m. Aug. 19 (gage height, $22.14 \mathrm{ft}$ ).

1928 to July 1955: Discharge, $45,200 \mathrm{cfs}$ Sept, 21,1938 , by computation of flow over dam.

Remarks.-Flood flow affected by Quabbin Reservolr (usable capacity, 55,700,000,000 cu ft) on Swift River, by diversion diversion from Ware River, and by dam failures on small tributaries. Dive station, by Quabbin Reservolr, and by smaller reservolrs.

Mean discharge, in cubic feet per second, August 1955

\begin{tabular}{c|c||r|r|r|r|r|r|r||r||r||r|}
\hline $\mathrm{Da}_{i}$ & Cfs & Day & Cis & Day & Cfs & Day & Cfs & Day & Cfs & Day & Cfs \\
\hline 1 & 368 & 7 & 47 & 12 & 380 & 17 & 980 & 22 & 7,360 & 27 & 3,050 \\
2 & 259 & 8 & 253 & 13 & 702 & 18 & 3,000 & 23 & 6,450 \\
3 & 253 & 9 & 331 & 14 & 2,580 & 19 & 30,600 & 24 & 5,740 & 28 & 2,570 \\
4 & 256 & 10 & 256 & 15 & 1,890 & 20 & 20,800 & 25 & 4,330 & 3,340 \\
5 & 256 & 11 & 246 & 16 & 1,400 & 21 & 10,800 & 26 & 3,610 \\
6 & 184 & & & & 31 & 1,920 \\
\hline
\end{tabular}

Gage height, in feet, and discharge, in cuble feet per second, at indicated time

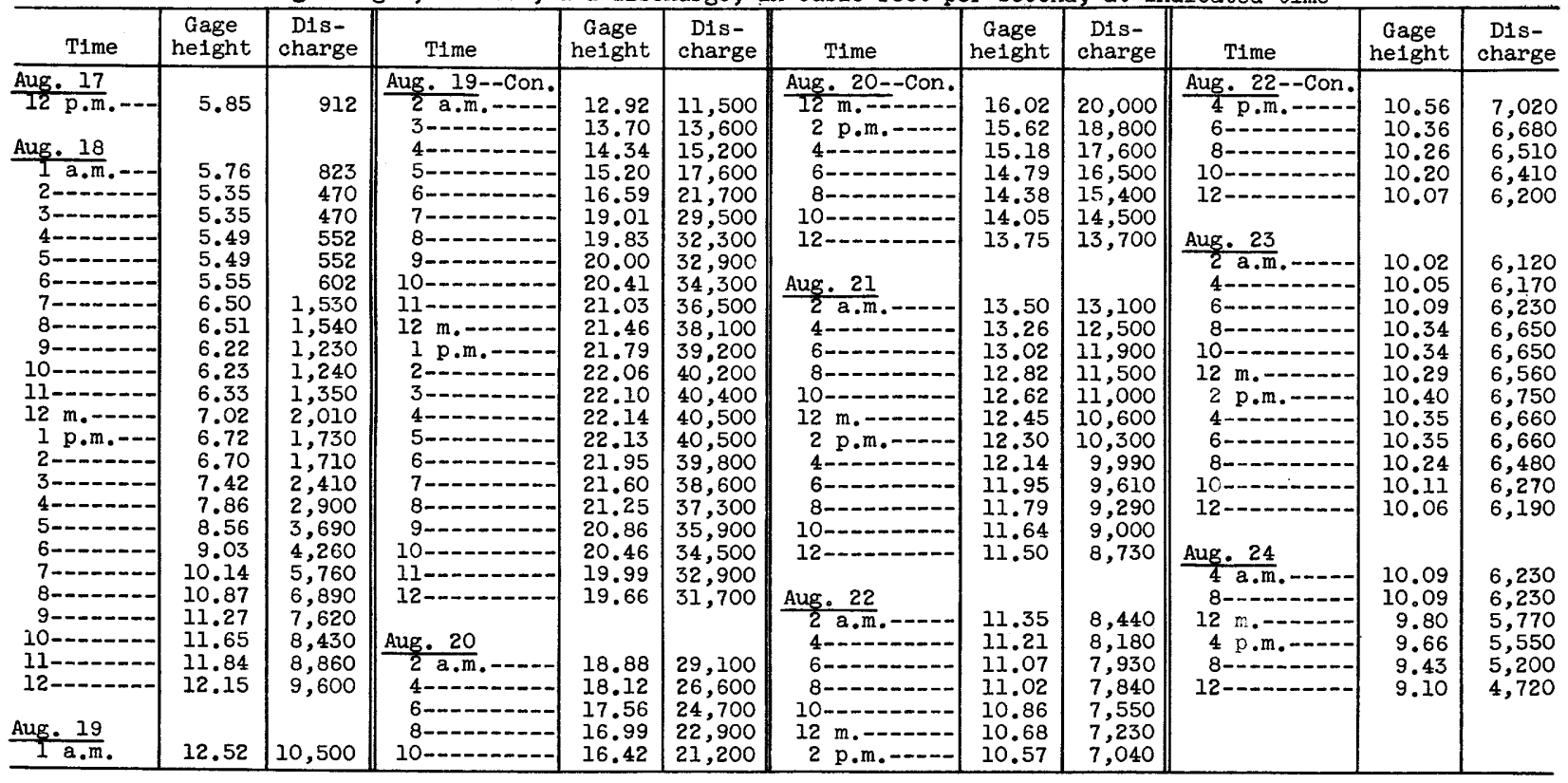


(71) Middle Branch Westfield River at Goss Heights, Mass.

Location. - Lat $42^{\circ} 15^{\prime} 31^{\prime \prime}$, long $72^{\circ} 52^{\prime} 23^{\prime \prime}$, on right bank at upstream side of highway bridge at Goss Heights, Hampshire County, 0.35 mile upstream from mouth and 1.7 miles north of Huntington. Datum of gage 1 s $400.30 \mathrm{ft}$ above mean sea level, datum of 1929.

Drainage area. $--52.6 \mathrm{sq} \mathrm{ms}$.

Gage-helght record.--Water-stage recorder graph.

Discharge record.--Stage-discharge relation defined by current-meter measurements below 5,700 cf's and extended to peak stage on basis of slope-area determination at gage height $11.33 \mathrm{ft}$.

Maxima.--August 1955: Discharge, 16,500 cfs 4 a.m. Aug. 19 (gage height, i1.33 ft)

from rating curve extended abre, 19,900 cfs Sept. 21, 1938 (gage height, 10.61 ft),

from rating curve extended above $3,200 \mathrm{cfs}$ on basis of mean of two contracted-opening determinations of peak
flow; maximum gage height, $13.87 \mathrm{ft}$ Mar. 12,1936 (ice Jam).

Mean discharge, in cubic feet per second, August 1955

\begin{tabular}{|c|c|c|c|c|c|c|c|c|c|c|c|}
\hline Day & $\mathrm{Cfs}$ & Day & Cfs & Day & $\mathrm{Cfs}$ & Day & $\mathrm{Cfs}$ & Day & $\mathrm{Cfs}$ & Day & Cfs \\
\hline $\begin{array}{l}1 \\
2 \\
3 \\
4 \\
5 \\
6\end{array}$ & $\begin{array}{l}2.4 \\
2.0 \\
1.8 \\
1.7 \\
1.6 \\
1.5\end{array}$ & $\begin{array}{r}7 \\
8 \\
9 \\
10 \\
11\end{array}$ & $\begin{array}{l}1.5 \\
1.4 \\
1.6 \\
2.0 \\
2.4\end{array}$ & $\begin{array}{l}12 \\
13 \\
14 \\
15 \\
16\end{array}$ & $\begin{array}{r}10 \\
329 \\
841 \\
211 \\
79\end{array}$ & $\begin{array}{l}17 \\
18 \\
19 \\
20 \\
21\end{array}$ & $\begin{array}{r}44 \\
1,400 \\
5,190 \\
578 \\
263\end{array}$ & $\begin{array}{l}22 \\
23 \\
24 \\
25 \\
26\end{array}$ & $\begin{array}{r}185 \\
154 \\
114 \\
85 \\
70\end{array}$ & $\begin{array}{l}27 \\
28 \\
29 \\
30 \\
31\end{array}$ & $\begin{array}{l}59 \\
51 \\
43 \\
39 \\
39\end{array}$ \\
\hline
\end{tabular}

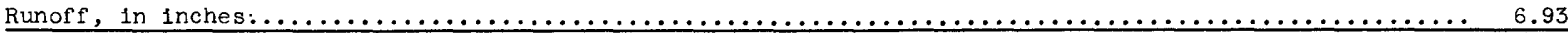

Gage height, in feet, and discharge, in cubic feet per second, at indicated time

\begin{tabular}{|c|c|c|c|c|c|c|c|c|c|c|c|}
\hline T1me & $\begin{array}{c}\text { Gage } \\
\text { height }\end{array}$ & $\begin{array}{l}\text { Dis- } \\
\text { charge }\end{array}$ & Time & $\begin{array}{c}\text { Gage } \\
\text { he1ght }\end{array}$ & $\begin{array}{l}\text { Dis- } \\
\text { charge }\end{array}$ & Time & $\begin{array}{c}\text { Gage } \\
\text { he1ght }\end{array}$ & $\begin{array}{c}\text { Dis- } \\
\text { charge }\end{array}$ & Time & $\begin{array}{c}\text { Gage } \\
\text { he1ght }\end{array}$ & $\begin{array}{l}\text { Dis- } \\
\text { charge }\end{array}$ \\
\hline 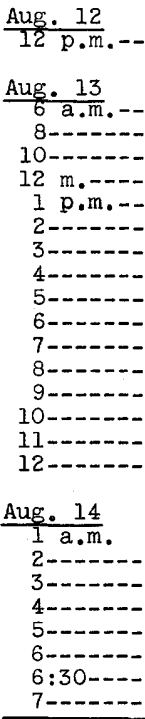 & $\begin{array}{l}1.47 \\
1.59 \\
1.79 \\
2.05 \\
2.29 \\
2.81 \\
2.97 \\
2.98 \\
3.05 \\
3.26 \\
3.52 \\
3.47 \\
3.30 \\
3.12 \\
2.99 \\
2.87\end{array}$ & $\begin{array}{r}55 \\
72 \\
109 \\
173 \\
247 \\
465 \\
545 \\
550 \\
585 \\
706 \\
864 \\
832 \\
730 \\
622 \\
555 \\
495\end{array}$ & 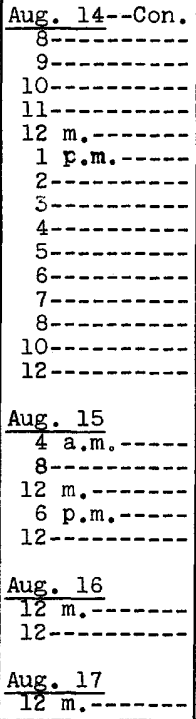 & $\begin{array}{l}4.36 \\
4.17 \\
3.95 \\
3.73 \\
3.57 \\
3.44 \\
3.28 \\
3.17 \\
3.09 \\
3.02 \\
2.99 \\
2.99 \\
2.95 \\
2.80 \\
2.65 \\
\\
2.42 \\
2.25 \\
2.12 \\
1.95 \\
1.79 \\
\\
1.61 \\
1.47\end{array}$ & $\begin{array}{r}1,490 \\
1,340 \\
1,160 \\
1,010 \\
900 \\
814 \\
718 \\
652 \\
605 \\
570 \\
555 \\
555 \\
535 \\
460 \\
389 \\
\\
\\
294 \\
234 \\
193 \\
146 \\
109 \\
\\
76 \\
55\end{array}$ & 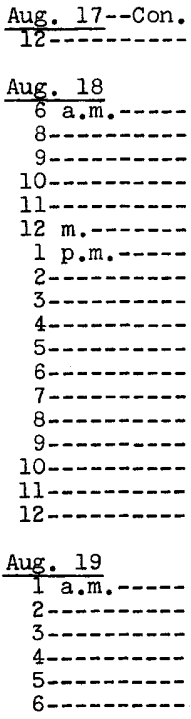 & $\begin{array}{r}1.30 \\
1.52 \\
1.70 \\
1.79 \\
2.68 \\
3.07 \\
5.00 \\
7.00 \\
7.18 \\
6.52 \\
6.03 \\
5.63 \\
5.23 \\
4.90 \\
4.62 \\
4.53 \\
4.50 \\
4.86 \\
\\
5.85 \\
7.47 \\
10.20 \\
11.33 \\
9.82 \\
8.50\end{array}$ & $\begin{array}{r}35 \\
62 \\
91 \\
109 \\
403 \\
595 \\
2,060 \\
4,740 \\
5,060 \\
3,960 \\
3,250 \\
2,740 \\
2,290 \\
1,960 \\
1,750 \\
1,630 \\
1,600 \\
1,920 \\
\\
3,020 \\
5,600 \\
12,600 \\
16,500 \\
12,900 \\
9,840\end{array}$ & 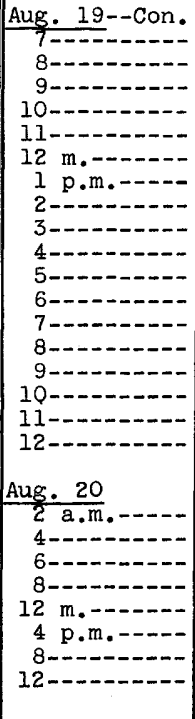 & $\begin{array}{l}7.50 \\
7.01 \\
6.75 \\
6.81 \\
6.76 \\
6.38 \\
5.90 \\
5.45 \\
4.95 \\
4.60 \\
4.32 \\
4.06 \\
3.81 \\
3.66 \\
3.50 \\
3.38 \\
3.25 \\
3.17\end{array}$ & $\begin{array}{r}7,640 \\
6,660 \\
6,140 \\
6,260 \\
6,160 \\
5,430 \\
4,570 \\
3,800 \\
3,020 \\
2,510 \\
2,150 \\
1,840 \\
1,560 \\
1,410 \\
1,260 \\
1,150 \\
1,040 \\
979\end{array}$ \\
\hline
\end{tabular}


(73) West Branch Westfield River at Huntington, Mass.

Location.--Lat $42^{\circ} 14^{\prime} 14^{\prime \prime}$, long $72^{\circ} 53^{\prime} 46^{\prime \prime}$, on left bank at Huntington, Hampshire County, 0.4 mile downstream from Roaring Brook and $1 \frac{1}{2}$ miles upstream from mouth. Datum of gage is $388.60 \mathrm{ft}$ above mean sea level, datum of 1929 . Drainage area. $--93.7 \mathrm{sq} \mathrm{mi}$.

Gage-height record.--Water-stage recorder graph.

Discharge record.--Stage-discharge relation defined by current-meter measurements below 9,500 cfs and extended to peak stage on basis of slope-area determination of peak flow. Shifting-control method used at times.

Maxima.--August 1955: Discharge, 26,100 cfs 4:30 a.m. Aug. 19 (gage height, $15.27 \mathrm{ft}$ ).

1935 to July 1955: Discharge, 21,800 cfs Sept. 21, i938 (gage height, $15.5 \mathrm{ft}$, from floodmarks), from rating curve extended above $1,900 \mathrm{cfs}$ on basis of computations of flow over dam at gage heights 11.93, 12.95, and 15.5 $\mathrm{ft}$ and slope-area determination at gage height $15.5 \mathrm{ft}$.

Remarks.--Some diurnal fluctuation at low flow caused by mill above station.

\begin{tabular}{r|r||r|r||r|r|r|r||r|r|r||r}
\hline Day & \multicolumn{1}{|c|}{ Cfs } & Day & Cfs & Day & Cfs & Day & Cfs & Day & Cfs & Day & Cfs \\
\hline 1 & 5.3 & 7 & 3.8 & 12 & 20 & 17 & 94 & 22 & 617 & 27 & 132 \\
2 & 4.8 & 8 & 3.5 & 13 & 554 & 18 & 2,260 & 23 & 481 & 28 & 114 \\
3 & 4.5 & 9 & 3.3 & 14 & 1,210 & 19 & 10,500 & 24 & 310 & 29 & 93 \\
4 & 4.2 & 10 & 4.0 & 15 & 341 & 20 & 1,390 & 25 & 211 & 30 & 81 \\
5 & 4.0 & 11 & 4.5 & 16 & 143 & 21 & 749 & 26 & 161 & 31 & 81 \\
6 & 3.8 & & & & & & & & & & \\
\hline
\end{tabular}

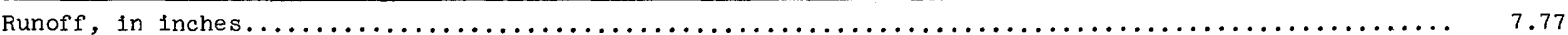

Gage height, in feet, and discharge, in cubic feet per second, at indicated time

\begin{tabular}{|c|c|c|c|c|c|c|c|c|c|c|c|}
\hline Time & $\begin{array}{c}\text { Gage } \\
\text { helght }\end{array}$ & $\begin{array}{l}\text { Dis- } \\
\text { charge }\end{array}$ & Time & $\begin{array}{c}\text { Gage } \\
\text { he1ght }\end{array}$ & $\begin{array}{l}\text { Dis- } \\
\text { charge }\end{array}$ & Time & $\begin{array}{c}\text { Gage } \\
\text { height }\end{array}$ & $\begin{array}{c}\text { D1s- } \\
\text { charge }\end{array}$ & me & $\begin{array}{c}\text { Gage } \\
\text { helght }\end{array}$ & $\begin{array}{c}\text { Dis- } \\
\text { charge }\end{array}$ \\
\hline$\frac{\text { Aug. } 11}{12}$ & & & Aug. 14-- & & & Aug. 1 & & & Aug. & & \\
\hline $12 \mathrm{p} \cdot \mathrm{m}$ & 0.34 & 4.8 & $10-\cdots$ & 3.74 & 1,450 & $1 p .1$ & 5.70 & 3,130 & $7-$ & 3.75 & 1,560 \\
\hline Aug. 1 & & & $12 \mathrm{~m}$ & $\begin{array}{l}3.60 \\
3.44\end{array}$ & 1,350 & & 7.45 & 5,210 & $8--$ & 3.68 & 1,490 \\
\hline$\frac{4 \cdot 1}{4 a \cdot 1}$ & .39 & 6.2 & $\begin{array}{rl}12 & \mathrm{~m} . \\
\mathrm{l} & \mathrm{p} .\end{array}$ & $\begin{array}{l}3.44 \\
3.32\end{array}$ & $\begin{array}{l}1,240 \\
1,150\end{array}$ & $\begin{array}{l}3-- \\
4--\end{array}$ & $\begin{array}{l}7.81 \\
7.65\end{array}$ & $\begin{array}{l}5,740 \\
5,480\end{array}$ & $9--$ & 3.61 & 1,440 \\
\hline $8--$ & .51 & 11 & $2-\cdots$ & 3.26 & 1,110 & & 7.06 & 4,690 & $11-$ & $\begin{array}{l}3.50 \\
3.50\end{array}$ & $\begin{array}{l}1,400 \\
1,350\end{array}$ \\
\hline $12 \mathrm{~m}$. & .63 & 17 & $3 \cdots-\cdots$ & 3.18 & 1,060 & & 6.80 & 4,390 & $12 \mathrm{~m}$ & 3.43 & 1,290 \\
\hline $3--$ & .66 & 19 & $4-\cdots$ & 3.09 & 993 & $7--$ & 6.48 & 3,990 & $1 \mathrm{p}$ & 3.36 & 1,240 \\
\hline $\begin{array}{l}6--1 \\
9--\end{array}$ & .81 & $\begin{array}{l}29 \\
39\end{array}$ & $5----$ & 3.01 & 937 & $8--$ & 6.16 & 3,640 & & $3 . ? 9$ & 1,180 \\
\hline $12-\cdots$ & $\begin{array}{r}.93 \\
1.14\end{array}$ & 39 & $6--\cdot--$ & 2.92 & 874 & $9--$ & 5.84 & 3,320 & & 3.23 & 1,130 \\
\hline $12--$ & 1.14 & 61 & $7---$ & 2.86 & 832 & $10--$ & 6.01 & 3,480 & $4--$ & 3.18 & 1,100 \\
\hline Aug. 13 & & & $9-$. & $\begin{array}{l}2.18 \\
2.71\end{array}$ & $\begin{array}{l}776 \\
727\end{array}$ & $11=-$ & 6.75 & 4,340 & $5--$ & 3.13 & 1,060 \\
\hline Ia. & 1.23 & 72 & $10 \ldots$ & $\begin{array}{l}2.11 \\
2.64\end{array}$ & $\begin{array}{l}678 \\
678\end{array}$ & $12-$ & 7.45 & 5,320 & $6--$ & 3.08 & $\begin{array}{l}1,030 \\
998\end{array}$ \\
\hline $2--$ & 1.27 & 78 & $11 \cdots$ & 2.57 & 630 & Aug. & & & & $\begin{array}{l}3.04 \\
3.01\end{array}$ & 977 \\
\hline $3--$ & 1.36 & 93 & $12--$ & 2.52 & 598 & $\frac{1}{\mathrm{a} \cdot \mathrm{m}}$ & 9.36 & 8,720 & $9=$ & 2.97 & 949 \\
\hline & 1.44 & 108 & & & & & 11.87 & 14,700 & $10-$ & 2.95 & 935 \\
\hline $5---$ & 1.50 & 121 & Aug. 15 & & & & 13.74 & 20,500 & $11-$ & 2.93 & 921 \\
\hline $6--$ & 1.57 & 138 & $6 \mathrm{a} \cdot \mathrm{m}$. & 2.24 & 419 & & 14.93 & 24,800 & $12=$ & $\begin{array}{l}. .90 \\
2.90\end{array}$ & 900 \\
\hline $7-\cdots$ & 1.67 & 165 & $12 \mathrm{mo}-$ & 2.05 & 315 & $4: 30$ & 15.27 & 26,100 & & 2.00 & (5) \\
\hline $8--$ & 1.84 & 222 & $6 \mathrm{p.m}$. & 1.88 & 237 & $5-1$ & 15.11 & 25,600 & Aug. 21 & & \\
\hline $9---$ & 1.95 & 268 & $12=--$ & 1.74 & 187 & $6-$ & 13.84 & 21,500 & $\frac{a}{2 \mathrm{a} \cdot \mathrm{m}}$ & 2.85 & 865 \\
\hline $10=-$ & 2.17 & 378 & & & & & 12.40 & 17,300 & $4-\ldots$ & $2.8 c$ & 844 \\
\hline $11=--$ & 2.34 & 481 & Aug. 16 & & & 8-- & 11.30 & 14,400 & $6--$ & 2.78 & 816 \\
\hline $12 \mathrm{~m} .--$ & 2.48 & 572 & $6 \mathrm{a} \cdot \mathrm{m}$ & 1.66 & 162 & & 10.43 & & & 2.75 & 795 \\
\hline 1 p.m. & 2.61 & 657 & $12 \mathrm{m.--}$ & 1.58 & 140 & $10 \ldots$ & 10.24 & 11,900 & $10-\ldots$ & 2.71 & 767 \\
\hline $2--$ & 2.76 & 762 & 6 p.m. & 1.51 & 123 & $11-\ldots$ & 9.91 & $11,200 \|$ & 1? m.- & 2.67 & 739 \\
\hline $3--$ & 2.93 & 881 & $12 \ldots$ & 1.44 & 108 & $12 \mathrm{~m} .-$ & 9.34 & 9,950 & $2 \mathrm{p}$. & 2.62 & 704 \\
\hline $4---$ & 3.15 & 1,040 & & & & & 8.68 & 8,600 & $4=-$ & 2.56 & 664 \\
\hline $5--$ & 3.20 & 1,070 & Aug. 17 & & & & 8.15 & 7,610 & $\epsilon--$ & 2.53 & 644 \\
\hline & 3.21 & 1,080 & $6 \mathrm{a} . \mathrm{m}$ & 1.39 & 98 & $3--$ & 7.54 & 6,510 & & 2.55 & 658 \\
\hline $\begin{array}{l}7---1 \\
8--1\end{array}$ & 3.13 & 1,020 & $12 \mathrm{m.--}$ & 1.35 & 92 & $4-\cdots$ & 7.03 & 5,690 & $10--$. & 2.58 & 677 \\
\hline $8---$. & 3.03 & 951 & 6 p.m. & 1.34 & 90 & $5--$ & 6.60 & 5,000 & $12--$ & 2.66 & 732 \\
\hline $\begin{array}{r}9-\cdots- \\
10---\end{array}$ & $\begin{array}{l}2.95 \\
2.91\end{array}$ & 895 & $12-\cdots$ & 1.31 & 85 & $6 \ldots$ & 6.22 & 4,420 & & & \\
\hline $10=-$ & 2.91 & 867 & & & & & & 3,880 & Aug. 22 & & \\
\hline $11--$ & 2.95 & 895 & Aug. 18 & & & $8--$. & 5.57 & 3,520 & & & \\
\hline $12---$ & 2.94 & 888 & I a.m. & 1.30 & 83 & $9--$ & 5.33 & 3,230 & $6-\cdots$ & 2.62 & 704 \\
\hline ug. 14 & & & & 1.29 & 81 & $10 \ldots$ & 5.07 & 2,910 & $12 \mathrm{m.-}$ & 2.52 & 638 \\
\hline & & & 4 & 1.29 & 81 & $11-\ldots$ & 4.86 & 2,680 & 6 p.m & 2.35 & 528 \\
\hline a.m. & 3.25 & 100 & 5 & 1.28 & 80 & $12 \ldots$ & 4.68 & 2,480 & $10-\cdots$ & 2.26 & 471 \\
\hline $3-$ & 3.32 & 1,150 & $6 \ldots$ & $\begin{array}{l}1.27 \\
1.27\end{array}$ & $\begin{array}{l}78 \\
78\end{array}$ & Aug. & & & & 2.25 & 465 \\
\hline $4---$ & 3.73 & 1,440 & $7-\ldots$ & 1.37 & 95 & $\frac{\mathrm{Aug} \cdot \mathrm{c}}{1 \mathrm{a} \cdot \mathrm{m}}$ & & $2,290 \|$ & Aug. 23 & & \\
\hline $5--$ & 4.58 & 2,120 & $8---5$ & 1.53 & 128 & $2-\cdots$ & 4.37 & 2,150 & $4 \mathrm{a} \cdot \mathrm{m}$ & 2.33 & 514 \\
\hline $6---$ & 4.82 & 2,340 & $9-\cdots$ & 1.73 & 183 & $3-\cdots$ & 4.2 & 2,000 & $8 \cdots$ & 2.36 & 534 \\
\hline 7--- & 4.55 & 2,100 & $10-\cdots$ & 2.14 & 364 & $4---$ & 4.08 & 1, & $12 \mathrm{m.-}$ & 2.33 & 514 \\
\hline 8--- & 4.19 & 1,780 & $11-\cdots$ & 3.22 & 1,080 & $5-\ldots$ & 3.96 & 1,740 & $6 \mathrm{p} . \mathrm{m}_{\mathrm{a}}$ & 2.22 & 447 \\
\hline & 3.96 & 1,600 & $12 \mathrm{m.}$ & 4.25 & 1,830 & $6 \ldots$ & 3.82 & 1,620 & $12 \cdots$ & 2.12 & 387 \\
\hline
\end{tabular}


(84) Westfield River near Westfield, Mass.

Location.--Lat $42^{\circ} 06^{\prime} 24^{\prime \prime}$, long $72^{\circ} 41^{\prime} 58^{\prime \prime}$, on left bank 0.7 mile downstream from Great Brook and 3 miles east of Westfield, Hampden County. Datum of gage is $98.25 \mathrm{ft}$ above mean sea level, datum of 1929 .

Drainage area. $-497 \mathrm{sq}$ m1. periods $5: 45 \mathrm{am}$. Aug. 19 to Aug. 21 and Aug. 23 to noon Aug. 24 based on outside-gage readings and floodmarks. Discharge record.--Stage-discharge relation defined by current-meter measurements below 18,000 cfs and extended to peak stage on basis of computations of flow over dam at gage heights $27.20,29.40$, and 34.2

Aug. 22 estimated on basis of trend of gage-he1ght graph. Backwater from grass at times.

Maxima.--August 1955: D1scharge, $70,300 \mathrm{cfs} 7$ to 8 a.m. Aug. 19 (gage helght, $34.2 \mathrm{ft}$ from floodmarks). computation of flow over dam.

Remarks.--Flood flow affected by Borden Brook and Cobble Mountain Reservoirs (combined usable capacity, 3,394,000,000 cuft) medium flows regulated by Borden Brook and Cobble Mountain Reservolrs. Diversion above station from Westrield medium flows regulated by Borden Brook and Cobble

Mean discharge, in cubic feet per second, August 1955

\begin{tabular}{c|r||r|r||r|r||r|r|r|r||r||r|}
\hline Day & Cfs & Day & Cfs & Day & Cf's & Day & Cfs & Day & Cfs & Day & Cfs \\
\hline 1 & 79 & 7 & 95 & 12 & 202 & 17 & 1,960 & 22 & 2,710 & 27 & 2,380 \\
2 & 110 & 8 & 79 & 13 & 1,300 & 18 & 7,210 & 23 & $3,040$. & 28 & 2,470 \\
3 & 95 & 9 & 83 & 14 & 3,140 & 19 & 37,400 & 24 & 3,200 & 29 & 3,490 \\
4 & 107 & 10 & 81 & 15 & 1,790 & 20 & 11,000 & 25 & 3,480 & 30 & 3,350 \\
5 & 114 & 11 & 101 & 16 & 2,230 & 21 & 3,620 & 26 & 2,740 & 31 & 2,580 \\
6 & 114 & & & & & & \\
\hline
\end{tabular}

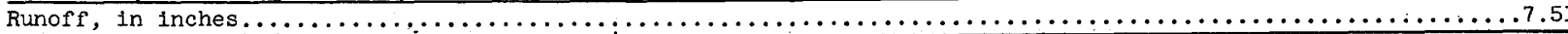

Gage height, in feet, and discharge, in cubic feet per second, at indicated time

\begin{tabular}{|c|c|c|c|c|c|c|c|c|c|c|c|}
\hline Time & $\begin{array}{l}\text { Gage } \\
\text { eight }\end{array}$ & $\begin{array}{l}\text { Dis- } \\
\text { harge }\end{array}$ & Time & & $\begin{array}{l}\text { Dis- } \\
\text { harge }\end{array}$ & Time & & $\begin{array}{c}\text { Dis- } \\
\text { charge }\end{array}$ & Time & & $\begin{array}{l}\text { Dis- } \\
\text { charge }\end{array}$ \\
\hline 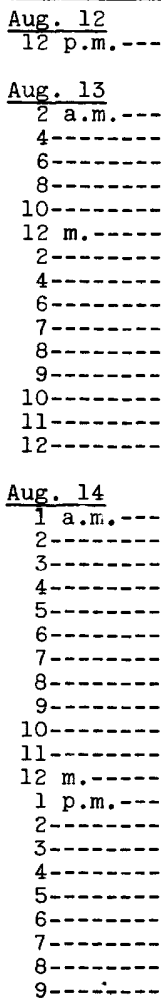 & $\begin{array}{l}7.47 \\
7.32 \\
6.99 \\
6.72 \\
6.61 \\
6.77 \\
7.04 \\
7.46 \\
8.10 \\
8.57 \\
8.71 \\
8.60 \\
8.33 \\
8.03 \\
7.77 \\
7.55 \\
7.43 \\
7.41 \\
7.31 \\
7.19 \\
7.05\end{array}$ & $\begin{array}{l}3,110 \\
2,940 \\
2,600 \\
2,340 \\
2,240 \\
2,380 \\
2,650 \\
3,100 \\
3,840 \\
4,430 \\
4,610 \\
4,470 \\
4,120 \\
3,760 \\
3,440 \\
3,200 \\
3,060 \\
3,040 \\
2,930 \\
2,800 \\
2,660\end{array}$ & 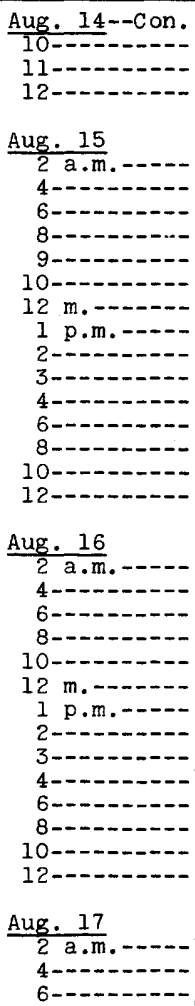 & $\begin{array}{l}6.54 \\
6.37 \\
6.20 \\
6.08 \\
6.09 \\
6.02 \\
5.86 \\
5.81 \\
5.85 \\
5.91 \\
5.92 \\
5.87 \\
5.82 \\
6.06 \\
6.46\end{array}$ & $\begin{array}{l}2,180 \\
2,020 \\
1,870 \\
1,760 \\
1,770 \\
1,710 \\
1,580 \\
1,540 \\
1,570 \\
1,620 \\
1,630 \\
1,590 \\
1,550 \\
1,740 \\
2,100\end{array}$ & 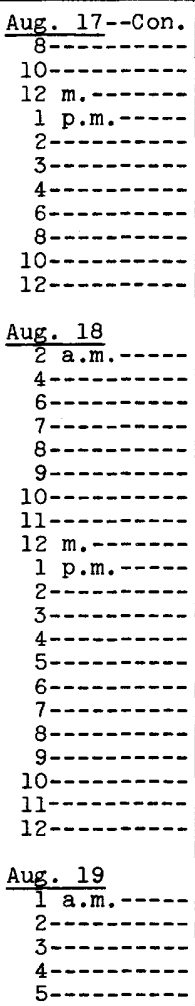 & $\begin{array}{r}6.49 \\
6.40 \\
6.30 \\
6.25 \\
6.28 \\
6.43 \\
6.25 \\
6.10 \\
5.88 \\
5.66 \\
5.42 \\
\\
\\
5.17 \\
4.98 \\
4.76 \\
4.72 \\
4.78 \\
4.87 \\
5.10 \\
5.95 \\
6.37 \\
7.10 \\
8.75 \\
10.90 \\
13.25 \\
14.77 \\
16.35 \\
16.99 \\
17.06 \\
16.78 \\
16.36 \\
16.04 \\
15.75 \\
\\
\end{array}$ & $\begin{array}{r}2,130 \\
2,050 \\
1,960 \\
1,920 \\
1,940 \\
2,080 \\
1,920 \\
1,780 \\
1,590 \\
1,420 \\
1,230 \\
\\
1,060 \\
932 \\
796 \\
772 \\
808 \\
862 \\
1,010 \\
1,650 \\
2,020 \\
2,710 \\
4,660 \\
7,810 \\
11,700 \\
14,500 \\
17,800 \\
19,200 \\
19,300 \\
18,700 \\
17,800 \\
17,100 \\
16,500 \\
\end{array}$ & 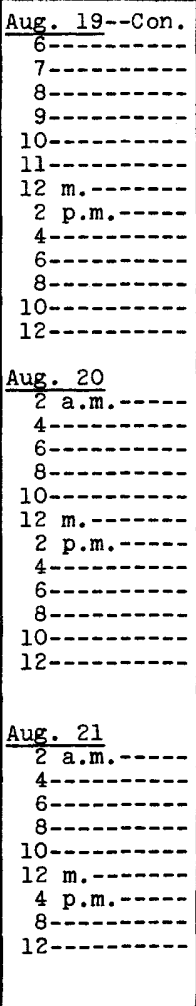 & $\begin{array}{l}9.00 \\
8.65 \\
8.37 \\
8.12 \\
7.90 \\
7.72 \\
7.42 \\
7.22 \\
7.09\end{array}$ & $\begin{array}{r}17,300 \\
15,000 \\
14,000 \\
12,600 \\
11,400 \\
10,300 \\
9,410 \\
8,450 \\
7,570 \\
6,820 \\
6,140 \\
5,550\end{array}$ \\
\hline
\end{tabular}


(85) Connecticut River at Thompsonvilie, Conn.

Location.--Lat $41^{\circ} 59^{\prime} 14^{\prime \prime}$, long $72^{\circ} 36^{\prime} 21^{\prime \prime}$, on right bank just upstream from Enfleld Dam and 1 mile downstream from Thompsonville, Hartford County. Datum of gage is $38.48 \mathrm{ft}$ above mean sea level, datum of 1929.

Drainage area.--9,661 sq $\mathrm{ml}$.

Gage-helght record.--Water-stage recorder graph.

Discharge record.-Stage-discharge relation defined by current-meter measurements.

Maxima.--August 1955: Discharge, 174,000 efs 7 p.m. Aug. 19 (gage he1ght, 10.93 ft).

1928 to July 1955: Discharge, $282,000 \mathrm{cfs}$ Mar. 20, 1936 (gage height, 16.6 ft, from floodmarks).

Remarks.--Flood flow affected by Quabbin Reservolr (usable capacity, 55,700,000,000 cu ft), Borden Brook and Cobble

Mountain Reservolrs (compined usable capacity, 3,394,000,000 cu ft), Knightville Reservolr (usable capacity,

$2,130,0 n 0,0 n 0 \mathrm{cu} f \mathrm{t}$ ), by other reservoirs, and by diversion from ware River. Discharge includes water diverted around station by canal of Connecticut Light \& Power Co. Low and medium flows regulated by powerplants, by di-

version from Chicopee River basin, and by reservoirs (combined usable capacity, about $88 \frac{1}{2}$ billion cu ft).

Mean discharge, in cubic feet per second, August 1955

\begin{tabular}{|c|c|c|c|c|c|c|c|c|c|c|c|}
\hline Day & Cfs & Day & Cf's & Day & Cfs & Day & Cfs & Day & $\mathrm{Cfs}$ & Day & $\mathrm{Cfs}$ \\
\hline $\begin{array}{l}1 \\
2 \\
3 \\
4 \\
5 \\
6\end{array}$ & $\begin{array}{l}2,460 \\
3,420 \\
3,400 \\
3,720 \\
2,620 \\
1,640\end{array}$ & $\begin{array}{r}7 \\
8 \\
9 \\
10 \\
11\end{array}$ & $\begin{array}{l}1,130 \\
1,800 \\
2,290 \\
2,550 \\
3,660\end{array}$ & $\begin{array}{l}12 \\
13 \\
14 \\
15 \\
16\end{array}$ & $\begin{array}{r}9,700 \\
15,400 \\
36,700 \\
45,600 \\
31,300\end{array}$ & $\begin{array}{l}17 \\
18 \\
19 \\
20 \\
21\end{array}$ & $\begin{array}{r}23,800 \\
29,200 \\
127,000 \\
104,000 \\
49,900\end{array}$ & $\begin{array}{l}22 \\
23 \\
24 \\
25 \\
26\end{array}$ & $\begin{array}{l}29,400 \\
25,700 \\
24,500 \\
24,800 \\
21,700\end{array}$ & $\begin{array}{l}27 \\
28 \\
29 \\
30 \\
31\end{array}$ & $\begin{array}{l}18,000 \\
11,100 \\
13,900 \\
14,900 \\
14,200\end{array}$ \\
\hline
\end{tabular}

Gage height, in feet, and discharge, in cublc feet per second, at indicated time

\begin{tabular}{|c|c|c|c|c|c|c|c|c|c|c|c|}
\hline $\mathrm{T} 1 \mathrm{~m}$ & & $\begin{array}{c}\text { D } \\
\text { che }\end{array}$ & ime & & & & & & Ime & & \\
\hline 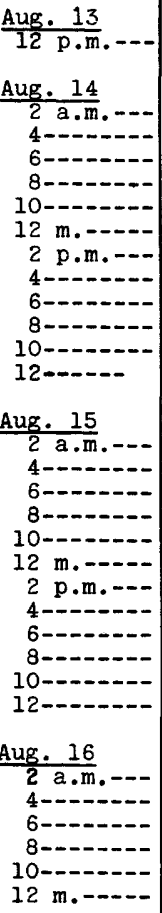 & $\begin{array}{l}2.59 \\
2.84 \\
3.07 \\
3.25 \\
3.42 \\
3.60 \\
3.75 \\
3.86 \\
3.94 \\
4.00 \\
4.05 \\
4.07\end{array}$ & $\begin{array}{l}22,600 \\
26,300 \\
30,000 \\
32,900 \\
35,700 \\
38,800 \\
41,400 \\
43,000 \\
44,500 \\
45,500 \\
46,400 \\
46,800\end{array}$ & 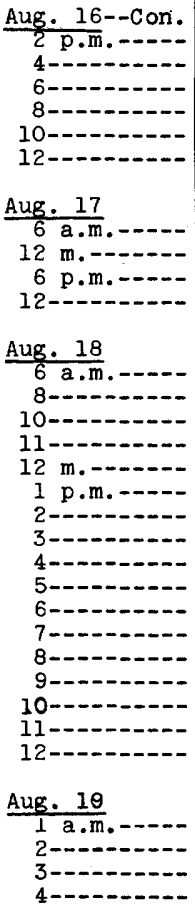 & $\begin{array}{l}2.45 \\
2.48 \\
2.54 \\
2.64 \\
2.76 \\
2.86 \\
2.96 \\
3.06 \\
3.16 \\
3.24 \\
3.38 \\
3.52 \\
3.67 \\
3.80 \\
3.92 \\
4.07 \\
4.22\end{array}$ & $\begin{array}{l}21,100 \\
21,400 \\
22,300 \\
23,700 \\
25,500 \\
27,100 \\
28,700 \\
30,200 \\
31,800 \\
33,000 \\
35,400 \\
37,800 \\
40,300 \\
42,600 \\
44,500 \\
47,300 \\
49,900\end{array}$ & 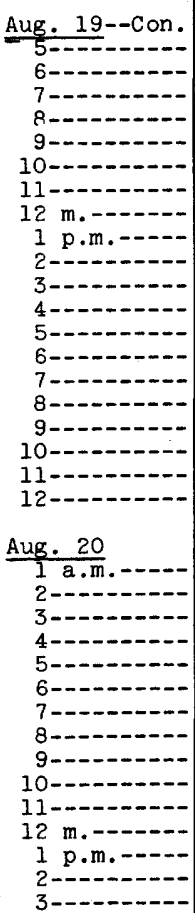 & $\begin{array}{r}5.53 \\
5.89 \\
6.35 \\
6.81 \\
7.51 \\
8.06 \\
8.77 \\
9.35 \\
9.84 \\
10.17 \\
10.44 \\
10.65 \\
10.82 \\
10.90 \\
10.93 \\
10.92 \\
10.84 \\
10.71 \\
10.56 \\
10.36 \\
\end{array}$ & $\begin{array}{r}74,800 \\
81,000 \\
90,400 \\
99,200 \\
112,000 \\
122,000 \\
135,000 \\
144,000 \\
153,000 \\
159,000 \\
164,000 \\
168,000 \\
172,000 \\
173,000 \\
174,000 \\
173,000 \\
172,000 \\
169,000 \\
167,000 \\
163,000\end{array}$ & 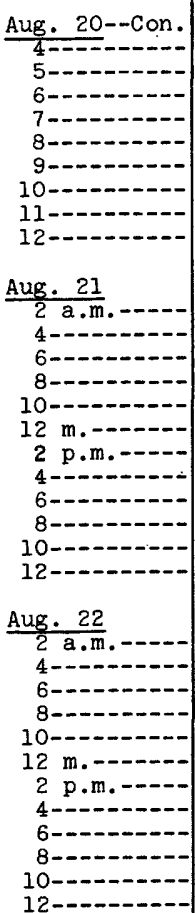 & $\begin{array}{l}3.46 \\
3.34 \\
3.22 \\
3.14 \\
3.09 \\
3.03 \\
2.94 \\
2.83 \\
2.78 \\
2.82\end{array}$ & $\begin{array}{l}36,000 \\
34,000 \\
32,000 \\
30,700 \\
29,900 \\
29,000 \\
27,500 \\
25,800 \\
25,100 \\
25,700 \\
25,400 \\
25,600\end{array}$ \\
\hline
\end{tabular}


(89) Scantic River at Broad Brook, Conn.

Location.--Lat $41^{\circ} 54 ' 42^{\prime \prime}$, long $72^{\circ} 33^{\prime} 48^{\prime \prime}$, on left bank 300 ft upstream from bridge on State Highway 140 , half a mile downstream from Broad Brook, I mile southwest of town of Broad Brook, Hartford County, and $8 \frac{1}{4}$ miles upstream from mouth. Datum of gage is $26.23 \mathrm{ft}$ above mean sea level, datum of 1929 .

Drainage area. $--98.4 \mathrm{sq} \mathrm{mi}$.

Gage-helght record.--Water-stage recorder graph except for periods 8 a.m. Aug. 19 to 1 p.m. Aug. 20 and 3 p.m. Aug. 24 to $9 \mathrm{a} . \mathrm{m}$. Aug. 27 . Graph for period $8 \mathrm{a} . \mathrm{m}$. Aug. 19 to $1 \mathrm{p.m}$. Aug. 20 drawn on basis of fioodmark and time of peak.

Discharge record--Stage-discharge relation defined by current-meter measurements below 1 ,200 cfs and by computations of flow over dams 7 and 9 miles above station at gage heights 13.9 and 14.4 ft, adjusted for flow from intervening area on basis of computation of flow over dam on Broad Brook, and by slope-area determination of peak flow. Discharge for Aug. 25, 26 estimated on basis of normal recession.

Maxima.--August 1955: Discharge, 13,300 cfs 6 p.m. Aug. 19 (gage helght, 19.9 ft, from floodmark). 1928 to July 1955: Discharge, $7,360 \mathrm{cfs}$ Sept. 21, 1938 (gage height, $16.08 \mathrm{ft}$, from floodmark).

\begin{tabular}{|c|c|c|c|c|c|c|c|c|c|c|c|}
\hline $\mathrm{Da}_{\mathrm{y}}$ & Cfs & $\mathrm{Da}$ & Cfs & Day & Cfs & Day & $\mathrm{Cfs}$ & Day & $\mathrm{Cfs}$ & Day & Cfs \\
\hline $\begin{array}{l}1 \\
2 \\
3 \\
4 \\
5 \\
6\end{array}$ & $\begin{array}{l}31 \\
31 \\
29 \\
28 \\
25 \\
28\end{array}$ & $\begin{array}{r}7 \\
8 \\
9 \\
10 \\
11\end{array}$ & $\begin{array}{l}29 \\
65 \\
62 \\
48 \\
48\end{array}$ & $\begin{array}{l}12 \\
13 \\
14 \\
15 \\
16\end{array}$ & $\begin{array}{r}93 \\
258 \\
403 \\
416 \\
233\end{array}$ & $\begin{array}{l}17 \\
18 \\
19 \\
20 \\
21\end{array}$ & $\begin{array}{r}114 \\
398 \\
7,770 \\
5,070 \\
1,460\end{array}$ & $\begin{array}{l}22 \\
23 \\
24 \\
25 \\
26\end{array}$ & $\begin{array}{l}839 \\
589 \\
567 \\
450 \\
360\end{array}$ & $\begin{array}{l}27 \\
28 \\
29 \\
30 \\
31\end{array}$ & $\begin{array}{l}324 \\
303 \\
282 \\
259 \\
246\end{array}$ \\
\hline
\end{tabular}

Gage height, in feet, and discharge, in cubic feet per second, at indicated time

\begin{tabular}{|c|c|c|c|c|c|c|c|c|c|c|c|}
\hline Time & $\begin{array}{c}\text { Gage } \\
\text { height }\end{array}$ & $\begin{array}{c}\text { Dis- } \\
\text { charge }\end{array}$ & Time & $\begin{array}{c}\text { Gage } \\
\text { height }\end{array}$ & $\begin{array}{c}\text { Dis- } \\
\text { charge }\end{array}$ & Time & $\begin{array}{c}\text { Gage } \\
\text { height }\end{array}$ & $\begin{array}{c}\text { Dis- } \\
\text { charge }\end{array}$ & Time & $\begin{array}{c}\text { Gage } \\
\text { height }\end{array}$ & $\begin{array}{l}\text { Dis- } \\
\text { charge }\end{array}$ \\
\hline 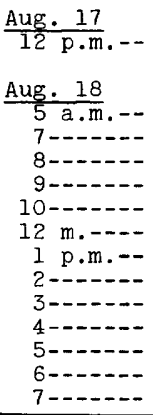 & $\begin{array}{l}1.21 \\
1.31 \\
1.31 \\
1.43 \\
2.05 \\
2.52 \\
2.86 \\
3.57 \\
4.33 \\
5.62 \\
6.07 \\
6.24 \\
6.35\end{array}$ & $\begin{array}{l}100 \\
113 \\
113 \\
129 \\
202 \\
278 \\
328 \\
407 \\
483 \\
645 \\
718 \\
748 \\
768\end{array}$ & 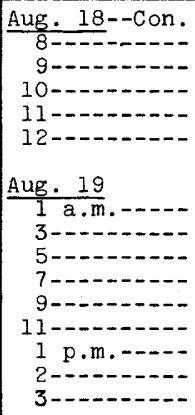 & $\begin{array}{l}7.95 \\
9.85 \\
11.00 \\
12.85 \\
14.6 \\
16.25 \\
17.9 \\
18.65 \\
19.1\end{array}$ & $\begin{array}{r}1,180 \\
1,840 \\
2,420 \\
3,600 \\
5,120 \\
7,050 \\
9,540 \\
10,900 \\
11,700\end{array}$ & 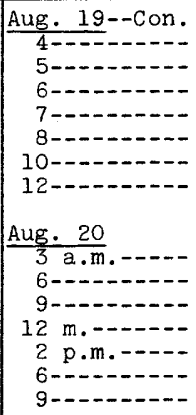 & $\begin{array}{l}19.5 \\
19.75 \\
19.9 \\
19.85 \\
19.8 \\
19.4 \\
18.8\end{array}$ & $\begin{array}{l}12,500 \\
13,000 \\
13,300 \\
13,200 \\
13,100 \\
12,300 \\
11,100\end{array}$ & 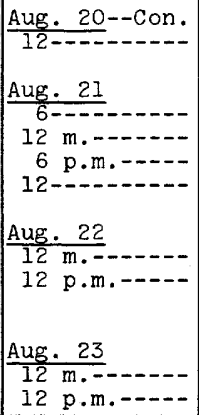 & $\begin{array}{l}9.72 \\
9.21 \\
8.68 \\
8.13\end{array}$ & $\begin{array}{l}1,650 \\
1,440 \\
1,230 \\
1,070\end{array}$ \\
\hline
\end{tabular}

(93) West Branch Farmington River near New Boston, Mass.

Location.--Lat $42^{\circ} 04^{\prime} 45^{\prime \prime}$, long $73^{\circ} \mathrm{O} 4^{\prime} 24^{\prime \prime}$, on left bank $5 \mathrm{ft}$ downstream from highway bridge, $0.3 \mathrm{mile}$ downstream from Clam River, and $i$ mile south of New Boston, Berkshire County. Datum of gage 1 s $758.2 i$ ft above mean sea level, datum of 1929 .

Drainage area.--92.0 $\mathrm{sq} \mathrm{mi}$.

Gage-height record.--Water-stage recorder graph.

Discharge record.--Stage-discharge relation defined by current-meter measurements below 9,600 cfs and extended to peak stage on basis of slope-area determination of peak flow.

Maxima.-August 1955: Discharge, 34,300 cfs 4:45 a.m. Aug. 19 (gage height, $14.06 \mathrm{ft}$ ).

1913 to July 1955: Discharge, 18,500 cfs Sept. 21 , ig38 (gage helght, 12.94 ft), from rating curve extended above $1,400 \mathrm{cfs}$ on basis of contracted-opening and slope-area determinations at gage helghts 10.65 and $12.94 \mathrm{ft}$. Remarks.--Flood flow affected by, and low and medium flows regulated by, otis Reservoir. Mean discharge, in cubic feet per second, August 1955

\begin{tabular}{|c|c|c|c|c|c|c|c|c|c|c|c|}
\hline Day & Cfs & Day & Cfs & Day & Cfs & Day & Cfs & Day & Cfs & Day & Cfs \\
\hline $\begin{array}{l}1 \\
2 \\
3 \\
4 \\
5 \\
6\end{array}$ & $\begin{array}{l}15 \\
14 \\
14 \\
14 \\
14 \\
16\end{array}$ & $\begin{array}{r}7 \\
8 \\
9 \\
10 \\
11\end{array}$ & $\begin{array}{l}16 \\
16 \\
15 \\
15 \\
16\end{array}$ & $\begin{array}{l}12 \\
13 \\
14 \\
15 \\
116\end{array}$ & $\begin{array}{r}34 \\
458 \\
1,340 \\
676 \\
337\end{array}$ & $\begin{array}{l}17 \\
18 \\
19 \\
20 \\
21\end{array}$ & $\begin{array}{r}178 \\
2,400 \\
16,100 \\
3,380 \\
1,710\end{array}$ & $\begin{array}{l}22 \\
23 \\
24 \\
25 \\
26\end{array}$ & $\begin{array}{r}1,160 \\
805 \\
504 \\
381 \\
302\end{array}$ & $\begin{array}{l}27 \\
28 \\
29 \\
30 \\
31\end{array}$ & $\begin{array}{l}272 \\
265 \\
223 \\
194 \\
171\end{array}$ \\
\hline
\end{tabular}


FLOOD DATA

(93) West Branch Farmington River near New Boston, Mass.--Continued

Gage helght, in feet, and discharge, in cubic feet per second, at indicated time

\begin{tabular}{|c|c|c|c|c|c|c|c|c|c|c|c|}
\hline & & & Time & & $\begin{array}{l}\text { Dis- } \\
\text { harge }\end{array}$ & Time & & arge & Time & & $\begin{array}{l}\text { Dis- } \\
\text { charge }\end{array}$ \\
\hline 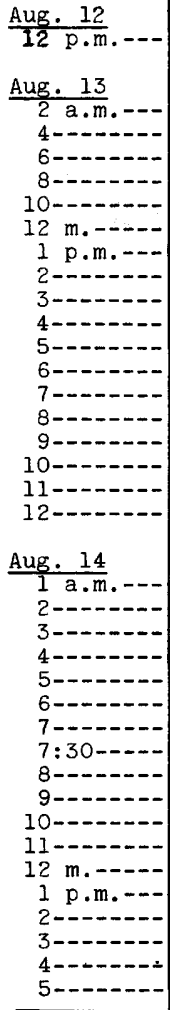 & $\begin{array}{l}5.40 \\
5.38 \\
5.39 \\
5.40 \\
5.58 \\
6.12 \\
6.92 \\
7.03 \\
7.02 \\
6.81 \\
6.58 \\
6.59 \\
6.46 \\
6.33 \\
6.23 \\
6.18 \\
6.11 \\
6.03\end{array}$ & $\begin{array}{r}850 \\
839 \\
844 \\
850 \\
958 \\
1,380 \\
2,150 \\
2,280 \\
2,260 \\
2,030 \\
1,800 \\
1,810 \\
1,680 \\
1,570 \\
1,480 \\
1,430 \\
1,370 \\
1,300\end{array}$ & 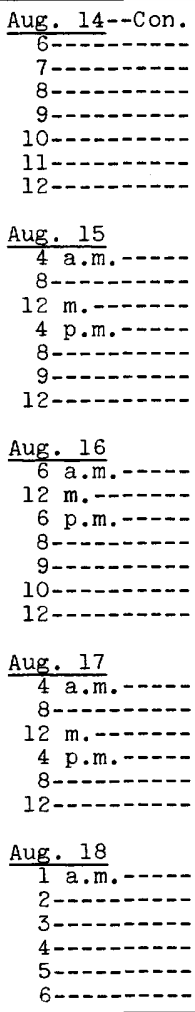 & $\begin{array}{l}4.39 \\
4.28 \\
4.15 \\
4.11 \\
4.06 \\
3.84 \\
3.80\end{array}$ & $\begin{array}{l}844 \\
746 \\
665 \\
595 \\
527 \\
472 \\
432 \\
\\
380 \\
343 \\
302 \\
290 \\
276 \\
217 \\
207\end{array}$ & 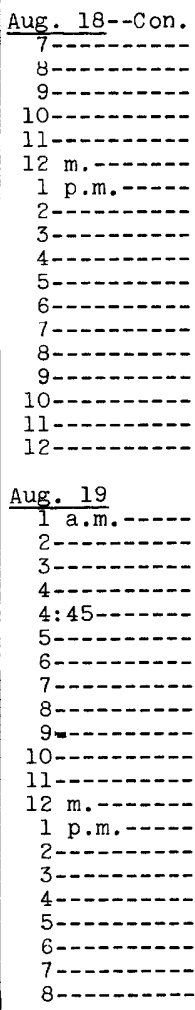 & $\begin{array}{r}11.22 \\
12.10 \\
12.84 \\
13.66 \\
14.06 \\
13.74 \\
13.35 \\
12.89 \\
12.63 \\
12.43 \\
12.17 \\
11.55 \\
11.12 \\
10.73 \\
10.42 \\
10.14 \\
9.38 \\
9.04 \\
9.44 \\
9.25 \\
9.10\end{array}$ & $\begin{array}{r}15,800 \\
20,600 \\
25,300 \\
31,200 \\
34,300 \\
31,800 \\
28,800 \\
25,600 \\
23,900 \\
22,600 \\
21,000 \\
17,500 \\
15,300 \\
13,500 \\
12,200 \\
11,000 \\
9,960 \\
9,010 \\
8,250 \\
7,540 \\
7,000\end{array}$ & 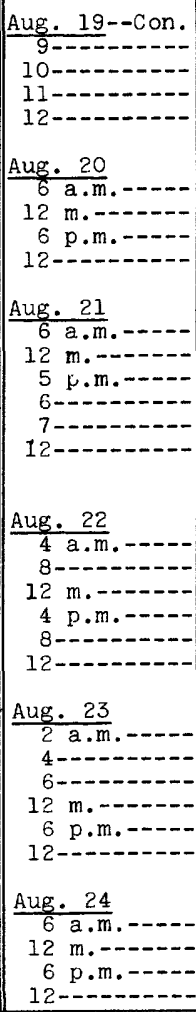 & $\begin{array}{l}5.65 \\
5.55 \\
5.45 \\
5.21 \\
5.01 \\
4.85\end{array}$ & $\begin{array}{l}1,720 \\
1,450 \\
1,220 \\
1,590 \\
2,400 \\
1,700\end{array}$ \\
\hline
\end{tabular}

(99) Still River at Robertsville, Conn.

Locat1on.--Lat $41^{\circ} 58^{\prime} 04^{\prime \prime}$, long $73^{\circ} 02^{\prime} 03^{\prime \prime}$, on left bank 1,500 ft downstream from Sandy Brook, 1 mile southeast of Robertsville, Litchfleld County, $1 \mathrm{mlle}$ northwest of Riverton, and 1 mile upstream from mouth. Datum of gage is $510.24 \mathrm{ft}$ above mean sea level, datum of 1929 .

Drainage area. $-84.4 \mathrm{sq} \mathrm{mi}$.

Gage-height record.--Water-stage recorder graph except for 5 to 11 a.m. on Aug. 19 during time of the peak. Graph for this period was drawn on basis of floodmark and time of peak.

Discharge record.--Stage-discharge relation defined by current-meter measurements below 5,600 cfs and by slope-area

determinations at gage heights 10.12 and $16.48 \mathrm{ft}$.

Maxima.--August 1955 ! Discharge, 44,000 of $8 \mathrm{a} . \mathrm{m}$. Aug. 19 (gage height, $16.48 \mathrm{ft}$, from floodmark).

I948 to July 1955: Discharge, $9,550 \mathrm{cf}$ s Dec. 31, 1948 (gage height, $10.12 \mathrm{ft}$ ). Mean discharge, in cubic feet per second, August 1955

\begin{tabular}{|c|c|c|c|c|c|c|c|c|c|c|c|}
\hline Day & Cfs & Day & Cfs & Day & Cfs & Day & Cfs & Day & Cfs & Day & Cfs \\
\hline $\begin{array}{l}1 \\
2 \\
3 \\
4 \\
5 \\
6\end{array}$ & $\begin{array}{c}6.8 \\
5.9 \\
6.1 \\
18 \\
14 \\
13 \\
\end{array}$ & $\begin{array}{r}7 \\
8 \\
9 \\
10 \\
11\end{array}$ & $\begin{array}{l}7.8 \\
5.7 \\
5.6 \\
5.9 \\
9.8\end{array}$ & $\begin{array}{l}12 \\
13 \\
14 \\
15 \\
16\end{array}$ & $\begin{array}{r}43 \\
331 \\
1,150 \\
592 \\
254\end{array}$ & $\begin{array}{l}17 \\
18 \\
19 \\
20 \\
21\end{array}$ & $\begin{array}{r}145 \\
2,490 \\
24,800 \\
3,710 \\
1,150\end{array}$ & $\begin{array}{l}22 \\
23 \\
24 \\
25 \\
26\end{array}$ & $\begin{array}{l}746 \\
629 \\
460 \\
324 \\
264\end{array}$ & $\begin{array}{l}27 \\
28 \\
29 \\
30 \\
31\end{array}$ & $\begin{array}{l}220 \\
202 \\
184 \\
140 \\
148\end{array}$ \\
\hline
\end{tabular}

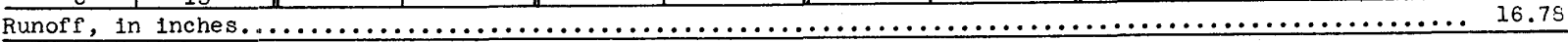

Gage height, in feet, and discharge, in cubic feet per second, at indicated time

\begin{tabular}{|c|c|c|c|c|c|c|c|c|c|c|c|}
\hline Time & $\begin{array}{c}\text { Gage } \\
\text { height }\end{array}$ & $\begin{array}{c}\text { Dis- } \\
\text { charge }\end{array}$ & T1me & $\begin{array}{c}\text { Gage } \\
\text { he1ght }\end{array}$ & $\begin{array}{l}\text { Dis- } \\
\text { charge }\end{array}$ & Time & $\begin{array}{c}\text { Gage } \\
\text { height }\end{array}$ & $\begin{array}{c}\text { Dis- } \\
\text { charge }\end{array}$ & TIme & $\begin{array}{c}\text { Gage } \\
\text { height }\end{array}$ & $\begin{array}{c}\text { Dis- } \\
\text { charge }\end{array}$ \\
\hline 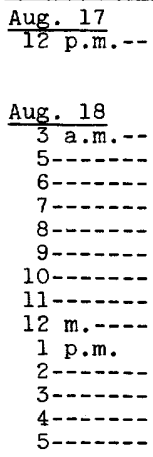 & $\begin{array}{l}1.43 \\
1.65 \\
1.68 \\
2.00 \\
3.20 \\
4.20 \\
4.80 \\
6.62 \\
6.57 \\
7.74 \\
7.48 \\
7.11 \\
6.96 \\
6.72\end{array}$ & $\begin{array}{r}75 \\
108 \\
113 \\
173 \\
620 \\
1,250 \\
1,700 \\
3,510 \\
3,450 \\
5,030 \\
4,640 \\
4,130 \\
3,940 \\
3,630\end{array}$ & 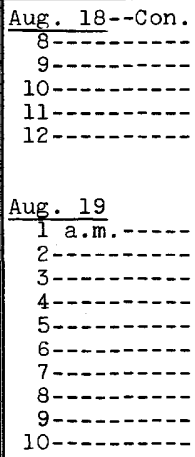 & $\begin{array}{l}11.45 \\
13.15 \\
14.05 \\
14.90 \\
15.58 \\
16.17 \\
16.43 \\
16.48 \\
16.40 \\
16.00\end{array}$ & $\begin{array}{l}13,800 \\
21,600 \\
26,700 \\
32,100 \\
36,900 \\
41,600 \\
43,600 \\
44,000 \\
43,400 \\
40,200\end{array}$ & 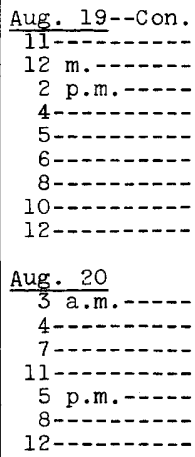 & $\begin{array}{r}15.43 \\
15.03 \\
13.72 \\
12.49 \\
11.90 \\
11.56 \\
10.54 \\
9.84 \\
9.33\end{array}$ & $\begin{array}{r}35,800 \\
33,000 \\
24,800 \\
18,200 \\
15,600 \\
14,200 \\
10,700 \\
8,800 \\
7,500\end{array}$ & 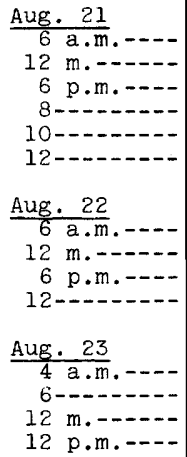 & $\begin{array}{l}5.31 \\
5.00 \\
4.59 \\
4.49 \\
4.49 \\
4.54\end{array}$ & $\begin{array}{r}1,380 \\
1,130 \\
854 \\
800 \\
800 \\
827\end{array}$ \\
\hline
\end{tabular}


(106) Burlington Brook near Burlington, Conn.

Location. - Lat $41^{\circ} 47^{\prime} 10^{\prime \prime}$, long $72^{\circ} 57^{\prime} 55^{\prime \prime}$, on left bank in rock gorge, $1 \frac{1}{4}$ miles north of Burlington, Hartford County, 3 miles upstream from mouth, and 3 miles southwest of Collinsvilie. Datum of gage is $714.00 \mathrm{ft}$ above mean sea level, datum of 1929 .

Drainage area. $--4.12 \mathrm{sq} \mathrm{mi}$.

Gage-height record.--Water-stage recorder graph.

Discharge record.--Stage-discharge relation defined by current-meter measurements below 320 cfs and by computations of flow through orffice and over weir and abutments at peak flow.

Maxima.--August 1955: Discharge, 1,720 cfs $4 \mathrm{a.m}$. Aug. 19 (gage height, $9.22 \mathrm{ft}$ ).

1931 to July 1955: Discharge, $676 \mathrm{cf}$ s Sept. 21, 1938 (gage helght, $7.24 \mathrm{ft}$ ).

Mean discharge, in cubic feet per second, August 1955

\begin{tabular}{|c|c|c|c|c|c|c|c|c|c|c|c|}
\hline Day & Cfs & Day & $\mathrm{Cfs}$ & Day & $\mathrm{Cf} s$ & Day & Cfs & Day & $\mathrm{Cfs}$ & Day & Cfs \\
\hline $\begin{array}{l}1 \\
2 \\
3 \\
4 \\
5 \\
6\end{array}$ & $\begin{array}{r}0.81 \\
.75 \\
.69 \\
.79 \\
.72 \\
1.06\end{array}$ & $\begin{array}{r}7 \\
8 \\
9 \\
10 \\
11\end{array}$ & $\begin{array}{r}2.80 \\
2.75 \\
.92 \\
.97 \\
1.99\end{array}$ & $\begin{array}{l}12 \\
13 \\
14 \\
15 \\
16\end{array}$ & $\begin{array}{l}8.98 \\
54 \\
53 \\
12.4 \\
5.77\end{array}$ & $\begin{array}{l}17 \\
18 \\
19 \\
20 \\
21\end{array}$ & $\begin{array}{c}3.57 \\
109 \\
677 \\
56 \\
27.0\end{array}$ & $\begin{array}{l}22 \\
23 \\
24 \\
25 \\
26\end{array}$ & $\begin{array}{l}17.8 \\
14.2 \\
11.9 \\
10.3 \\
10.4\end{array}$ & $\begin{array}{l}27 \\
28 \\
29 \\
30 \\
31\end{array}$ & $\begin{array}{l}8.95 \\
8.0 \\
7.13 \\
6.85 \\
7.13\end{array}$ \\
\hline
\end{tabular}

Gage height, in feet, and discharge, in cubic feet per second, at indicated time

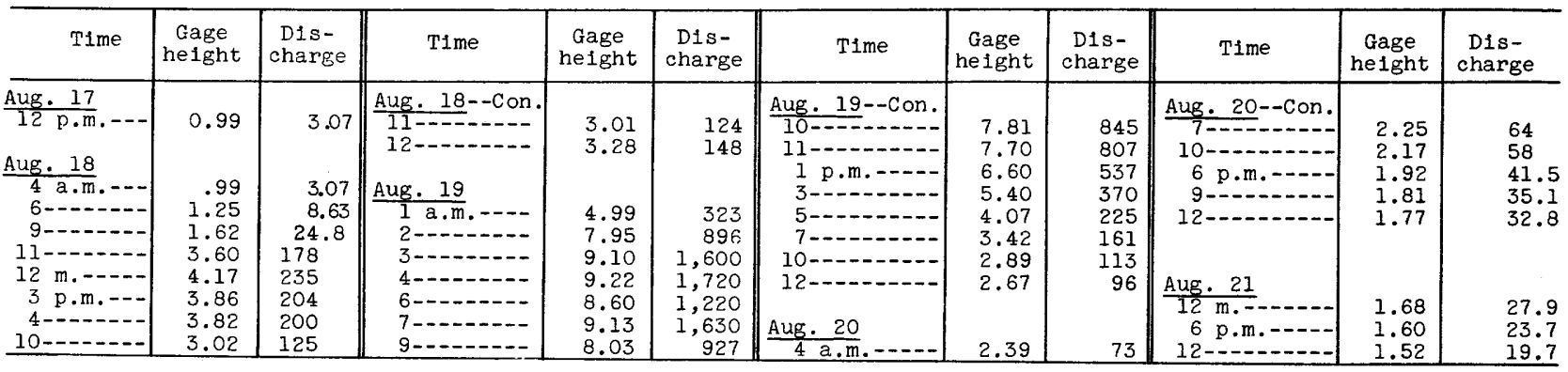

(111) Farmington River at Rainbow, Conn.

Location.--Lat $41^{\circ} 54^{\prime} 41^{\prime \prime}$, long $72^{\circ} 41^{\prime} 16^{\prime \prime}$, on left bank at Rainbow, Hartford County, 300 ft from Stevens Paper Mill,

.4 mile downstream from Farmington River Power Co. Dam, 1.3 miles upstream from Poquonock, 6.4 miles downstream

from Salmon Brook and 8 miles upstream from mouth. Datum of gage is $35.36 \mathrm{ft}$ above mean sea level, datum of 1929 . Drainage area. $-584 \mathrm{sq} \mathrm{mi}$.

Gage-height.- Water-stage recorder graph 9 a.m. to 12 p.m. Aug. 31 only.

Discharge record.--Discharges computed from powerplant data furnished by Farmington River Power Co., and determjnation of peak flow over dam for flood of August 19.

Maxima.--August 1955: D1scharge, 69,200 cfs $10-11$ p.m. Aug. 19 (gage height, 23.5 ft, from floodmarks). 1928 to July 1955: Discharge, 29,900 cfs Sept. 22, 1938 ; gage he1ght, $13.83 \mathrm{ft}$ Jan. $1,1949$.

Remarks.--Flood flow regulated by powerplants, by otis, Barkhamsted, East Branch, Nepaug and Whigville Reservolrs

having a combined capacity of about 6,450,000,000 cubic feet, and by diversions for domestic water supply from

Barkhamsted, Nepaug, and Whigville Reservoirs and Whites Bridge pumping plant.

\begin{tabular}{|c|c|c|c|c|c|c|c|c|c|c|c|}
\hline Day & Cfs & Day & $\mathrm{Cfs}$ & Day & Cf's & Day & Cfs & Day & Cfs & Day & $\mathrm{Cfs}$ \\
\hline $\begin{array}{l}1 \\
2 \\
3 \\
4 \\
5 \\
6\end{array}$ & $\begin{array}{l}215 \\
205 \\
215 \\
550 \\
300 \\
230\end{array}$ & $\begin{array}{r}7 \\
8 \\
9 \\
10 \\
11\end{array}$ & $\begin{array}{l}290 \\
420 \\
230 \\
230 \\
310\end{array}$ & $\begin{array}{l}12 \\
13 \\
14 \\
15 \\
16\end{array}$ & $\begin{array}{r}805 \\
1,700 \\
2,500 \\
4,500 \\
2,600\end{array}$ & $\begin{array}{l}17 \\
18 \\
19 \\
20 \\
21\end{array}$ & $\begin{array}{r}1,800 \\
1,850 \\
45,100 \\
56,700 \\
27,600\end{array}$ & $\begin{array}{l}22 \\
23 \\
24 \\
25 \\
26\end{array}$ & $\begin{array}{r}13,800 \\
8,460 \\
5,520 \\
4,870 \\
2,500\end{array}$ & $\begin{array}{l}27 \\
28 \\
29 \\
30 \\
31\end{array}$ & $\begin{array}{l}1,600 \\
1,450 \\
1,250 \\
1,450 \\
1,250\end{array}$ \\
\hline
\end{tabular}

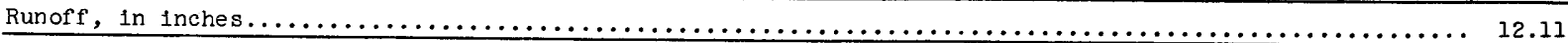

Gage height, in feet, and discharge, in cubic feet per second, at indicated time

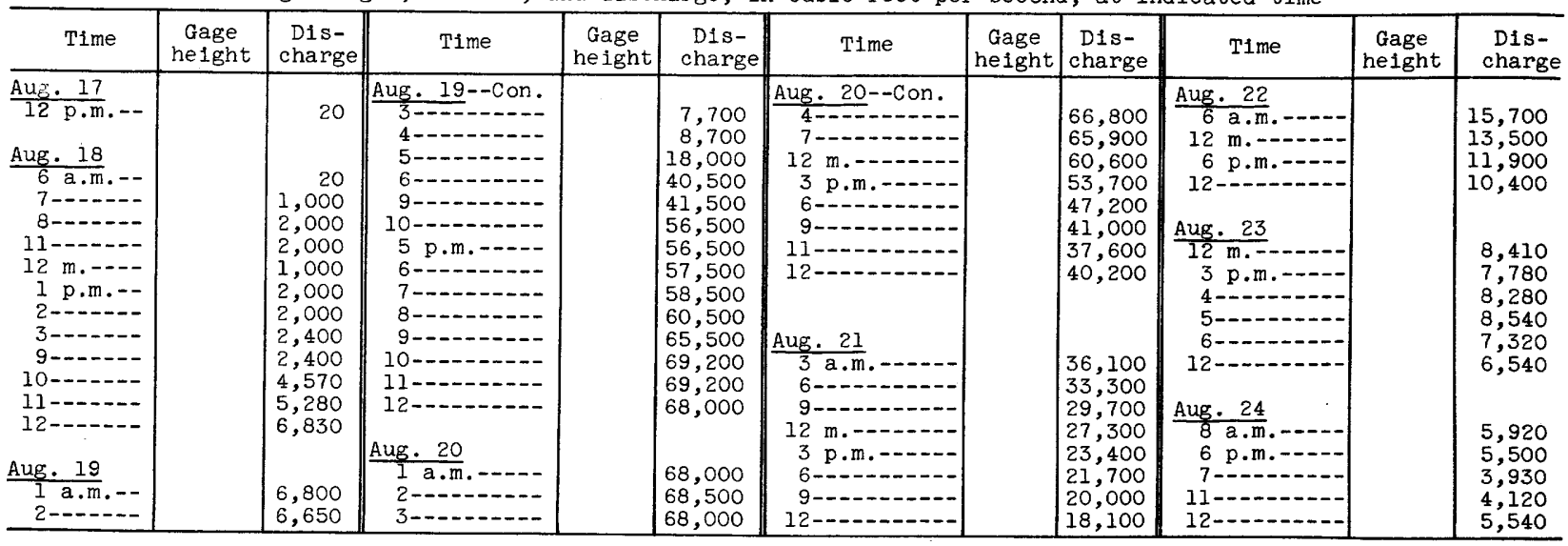


(129) Housatonic River at Gaylordsville, Conn.

Location.--Lat $41^{\circ} 39^{\prime} 11^{\prime \prime}$, long $73^{\circ} 29^{\prime} 25^{\prime \prime}$, on left bank 0.4 mile downstream from hydroelectric plant of connecticut Light \& Power Co., 0.5 mile upstream from bridge on U. S. Highwav 7 at Gavlordsville, Litchfield County, $1 \frac{1}{2}$ miles downstream from Tenmile River, and at mile 50.6. Datum of gage 1 s 236.78 feet above mean sea level, datum of 1929 .

Drainage area. $--994 \mathrm{sq} \mathrm{m}$.

Gage-helght record.--Water-stage recorder graph.

D1scharge record.--Stage-discharge relation defined by current-meter measurements below $28,000 \mathrm{cfs}$ and extended to peak stage on basis of logarithmic plotting.

Maxima.--August 1955: Discharge, 51,800 cfs 5 p.m. Aug. 19 (gage height, $18.58 \mathrm{ft}$ ).

1900-1914, 1940 to July 1955: D1scharge, 32,300 cfs Jan. 1, 1949 (gage helght, $14.85 \mathrm{ft}$ ).

Flood of May 1854 reached a stage of $21 \mathrm{ft} 3 \mathrm{in}$, at former gage site 0.6 mile downstream at different datum, reported by observer in 1902 . Flood of Sept. 22, 1938, reached a stage of $14.5 \mathrm{ft}$., from floodmarks, at present site (discharge, $37,000 \mathrm{cfs}$, by computation of peak flow over dam $2 \frac{1}{2}$ miles upstream adjusted for flow from intervening area).

Remarks.--Low and medium flows regulated by powerplants above station.

Mean discharge, in cubic feet per second, August 1955

\begin{tabular}{|c|c|c|c|c|c|c|c|c|c|c|c|}
\hline Day & Cfs & Day & Cfs & Day & Cfs & Day & Cfs & Day & Cfs & Day & $\mathrm{Cfs}$ \\
\hline $\begin{array}{l}1 \\
2 \\
3 \\
4 \\
5 \\
6\end{array}$ & $\begin{array}{l}227 \\
208 \\
206 \\
231 \\
299 \\
385\end{array}$ & $\begin{array}{r}7 \\
8 \\
9 \\
10 \\
11\end{array}$ & $\begin{array}{l}146 \\
157 \\
177 \\
145 \\
247\end{array}$ & $\begin{array}{l}12 \\
13 \\
14 \\
15 \\
16\end{array}$ & $\begin{array}{r}328 \\
955 \\
3,130 \\
3,240 \\
2,250\end{array}$ & $\begin{array}{l}17 \\
18 \\
19 \\
20 \\
21\end{array}$ & $\begin{array}{r}1,560 \\
1,820 \\
38,800 \\
27,700 \\
15,100\end{array}$ & $\begin{array}{l}22 \\
23 \\
24 \\
25 \\
26\end{array}$ & $\begin{array}{l}9,490 \\
7,460 \\
5,520 \\
4,040 \\
3,120\end{array}$ & $\begin{array}{l}27 \\
28 \\
29 \\
30 \\
31\end{array}$ & $\begin{array}{l}2,600 \\
2,320 \\
2,020 \\
1,850 \\
1,710\end{array}$ \\
\hline
\end{tabular}

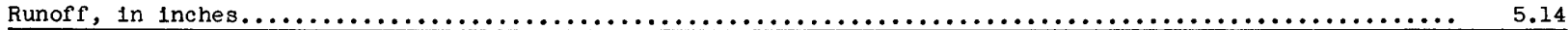

Gage height, in feet, and discharge, in cubic feet per second, at indicated time

\begin{tabular}{|c|c|c|c|c|c|c|c|c|c|c|c|}
\hline Time & $\begin{array}{c}\text { Gage } \\
\text { height }\end{array}$ & $\begin{array}{l}\text { Dis- } \\
\text { charge }\end{array}$ & Time & $\begin{array}{c}\text { Gage } \\
\text { height }\end{array}$ & $\begin{array}{l}\text { Dis- } \\
\text { charge }\end{array}$ & Time & $\begin{array}{c}\text { Gage } \\
\text { he1ght }\end{array}$ & $\begin{array}{l}\text { Dis- } \\
\text { charge }\end{array}$ & Time & $\begin{array}{c}\text { Gage } \\
\text { height }\end{array}$ & $\begin{array}{l}\text { Dis- } \\
\text { charge }\end{array}$ \\
\hline$\frac{\text { Aug. } 17}{12 \text { p.m. }}$ & 3.41 & 1,380 & $\frac{\text { Aug. } 19}{2 \cdot \mathrm{a} \cdot \mathrm{m}}$ & 8.95 & 10,700 & $\begin{array}{l}\text { Aug. } 20--C o n . \\
12 \text { m. }\end{array}$ & 13.56 & 26.200 & $\frac{\text { Aug. } 23}{4 a^{2}}$ & 7.80 & \\
\hline Aug. 18 & & & 4--.-- & $\begin{array}{l}11.75 \\
14.76\end{array}$ & $\begin{array}{l}19,000 \\
31,800\end{array}$ & $\begin{array}{r}6 \text { p.m. } \\
\end{array}$ & $\begin{array}{l}12.58 \\
11.81\end{array}$ & $\begin{array}{l}22,100 \\
19,200\end{array}$ & 4 p.m.-... & $\begin{array}{l}7.00 \\
7.72 \\
7.43\end{array}$ & \\
\hline $1 \mathrm{a} \cdot \mathrm{m}$ & $\begin{array}{l}3.20 \\
3.22\end{array}$ & $1, ? .10$ & $8-\cdots-$ & 16.52 & 40,600 & & & & $12=\ldots$ & 7.06 & 6,490 \\
\hline $4--$ & $\begin{array}{l}3.22 \\
2.56\end{array}$ & $\begin{array}{l}1,230 \\
746\end{array}$ & $10-10-10-10$ & 17.38 & 45,200 & Aug. 21 & & & & & \\
\hline $5-1$ & 2.52 & $\begin{array}{l}746 \\
722\end{array}$ & & 17.91 & 48,000 & $8 \mathrm{a} \cdot \mathrm{m}, \ldots$ & 10.95 & 16,300 & & & \\
\hline-- & 2.90 & 980 & 4 & $\begin{array}{l}18.23 \\
18.54\end{array}$ & $\begin{array}{l}49,800 \\
51,500\end{array}$ & 4 p.m. & 10.08 & 13,700 & Aug. 24 & & \\
\hline $7-\cdots$ & 3.21 & 1,220 & $5 \ldots$ & 18.58 & 51,800 & & & & 12 p.m. & $\begin{array}{l}6.52 \\
6.08\end{array}$ & $\begin{array}{l}5,490 \\
4,730\end{array}$ \\
\hline 10-10-10-1 & 3.27 & 1,270 & $6 \ldots$ & 18.53 & 51,500 & 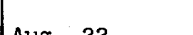 & & & & & \\
\hline 2 p.m. & $\begin{array}{l}3.48 \\
3.50\end{array}$ & $\begin{array}{l}1,440 \\
1,460\end{array}$ & 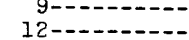 & $\begin{array}{l}17.78 \\
16.65\end{array}$ & 47,300 & $\frac{\text { Aug } \cdot 22}{8 \cdot 2 \cdot m}$ & & & 25 & & \\
\hline & 4.26 & 2,230 & & & & 4 p.m. & 8.15 & & $12 \mathrm{~m} .-$ & 5.65 & 4,040 \\
\hline $12-$ & $\begin{array}{l}4.35 \\
6.24\end{array}$ & $\begin{array}{l}2,300 \\
5,000\end{array}$ & $\frac{\text { Aug. } 20}{6 \cdot 2 \cdot m}$ & 14.86 & 32,300 & $\begin{array}{r}8-2-1 \\
12-0\end{array}$ & $\begin{array}{l}7.92 \\
7.80\end{array}$ & $\begin{array}{l}8,270 \\
8,010\end{array}$ & 12 p.m.n- & 5.23 & 3,430 \\
\hline
\end{tabular}

(137) Pomperaug River at Southbury, Conn.

Location.--Lat $41^{\circ} 28^{\prime} 50^{\prime \prime}$, long $73^{\circ} 13 \cdot 30^{\prime \prime}$, on right bank $200 \mathrm{ft}$ upstream from highway bridge, 800 ft downstream from Bullet Hill Brook, 0.6 mile west of Southbury, New Haven County, and 5.8 miles upstream from mouth. Datum of

gage is $165.60 \mathrm{ft}$ above mean sea level, datum of 1929 (levels by Corps of Englneers).

Drainage area. $--75.3 \mathrm{sq} \mathrm{ml}$.

Gag-helght record.--Water-stage recorder graph except for period 1 a.m. to 7 p.m. Aug. 19. Graph for this period drawn on basis of floodmark and time of peak.

Discharge record.--Stage-discharge relation defined by current-meter measurements below 1,200 cfs and by computation

of flow over dam at gage helght $16.0 \mathrm{ft}$ and slope-area determination of peak.

Maxima.--August 1955: Discharge, 29,400 cfs 6:30 a.m. Aug. 19 (gage height, $21.8 \mathrm{ft}, \mathrm{from}$ floodmarks).

1932 to July 1955: D1scharge, 7,420 ofs Sept. 21, 1938 (gage height, 16.0 ft, from floodmarks).

Remarks.--Infrequent regulation at low flow only by mili above station.

Mean discharge, in cubic feet per second, August 1955

\begin{tabular}{|c|c|c|c|c|c|c|c|c|c|c|c|}
\hline Day & Cfs & Day & Cfs & Day & $\mathrm{Cfs}$ & Day & Cfs & Day & $\mathrm{Cfs}_{\mathrm{s}}$ & Day & Cfs \\
\hline $\begin{array}{l}1 \\
2 \\
3 \\
4 \\
5 \\
6\end{array}$ & $\begin{array}{l}15 \\
14 \\
13 \\
12 \\
12 \\
12\end{array}$ & $\begin{array}{r}7 \\
8 \\
9 \\
10 \\
11\end{array}$ & $\begin{array}{l}13 \\
21 \\
16 \\
14 \\
15\end{array}$ & $\begin{array}{l}12 \\
13 \\
14 \\
15 \\
16\end{array}$ & $\begin{array}{r}60 \\
380 \\
1,360 \\
348 \\
181\end{array}$ & $\begin{array}{l}17 \\
18 \\
19 \\
20 \\
21\end{array}$ & $\begin{array}{r}126 \\
1,550 \\
9,510 \\
1,370 \\
679\end{array}$ & $\begin{array}{l}22 \\
23 \\
24 \\
25 \\
26\end{array}$ & $\begin{array}{l}468 \\
350 \\
270 \\
226 \\
193\end{array}$ & $\begin{array}{l}27 \\
28 \\
29 \\
30 \\
31\end{array}$ & $\begin{array}{l}88 \\
73 \\
56 \\
48 \\
48\end{array}$ \\
\hline
\end{tabular}

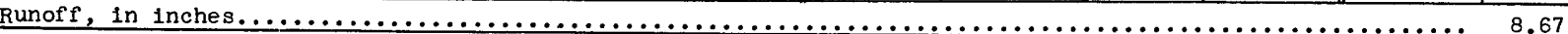

Gage helght, in feet, and discharge, in cubic feet per second, at indicated time

\begin{tabular}{|c|c|c|c|c|c|c|c|c|c|c|c|}
\hline Time & $\begin{array}{c}\text { Gatre } \\
\text { helght }\end{array}$ & $\begin{array}{c}\text { Dis- } \\
\text { charge }\end{array}$ & Time & $\begin{array}{c}\text { Gage } \\
\text { helght }\end{array}$ & $\begin{array}{c}\text { Dis- } \\
\text { charge }\end{array}$ & Time & $\begin{array}{c}\text { Gage } \\
\text { height }\end{array}$ & $\begin{array}{c}\text { Dis- } \\
\text { cnarge }\end{array}$ & Time & $\begin{array}{c}\text { Gage } \\
\text { helght }\end{array}$ & $\begin{array}{c}\text { Dis- } \\
\text { charge }\end{array}$ \\
\hline 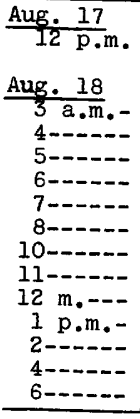 & $\begin{array}{l}3.47 \\
3.80 \\
4.52 \\
5.40 \\
5.87 \\
6.52 \\
7.45 \\
7.90 \\
9.23 \\
9.94 \\
9.75 \\
8.84 \\
8.02\end{array}$ & $\begin{array}{r}106 \\
165 \\
344 \\
680 \\
905 \\
1,230 \\
1,710 \\
1,960 \\
2,700 \\
3,120 \\
3,010 \\
2,470 \\
2,020 \\
\end{array}$ & 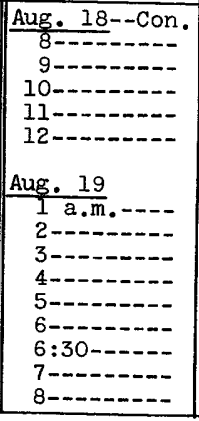 & $\begin{array}{l}10.00 \\
11.25 \\
13.2 \\
17.3 \\
19.5 \\
21.65 \\
21.8 \\
21.65 \\
20.9\end{array}$ & $\begin{array}{r}1,660 \\
1,540 \\
1,670 \\
2,150 \\
2,630 \\
\\
3,160 \\
3,960 \\
5,500 \\
9,500 \\
13,000 \\
24,600 \\
29,400 \\
28,100 \\
23,600\end{array}$ & 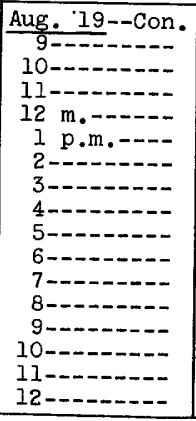 & $\begin{array}{l}19.95 \\
19.0 \\
18.05 \\
17.15 \\
16.0 \\
15.0 \\
14.0 \\
12.95 \\
11.85 \\
11.0 \\
10.35 \\
9.80 \\
9.35 \\
9.03 \\
8.73 \\
8.43\end{array}$ & $\begin{array}{l}19,800 \\
16,600 \\
13,800 \\
11,600 \\
9,300 \\
7,700 \\
6,440 \\
5,360 \\
4,400 \\
3,800 \\
3,370 \\
3,040 \\
2,770 \\
2,580 \\
2,410 \\
2,250 \\
\end{array}$ & 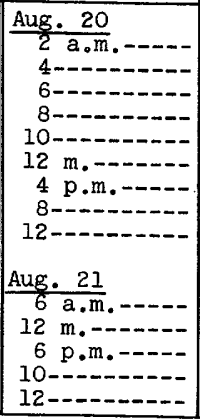 & $\begin{array}{l}7.93 \\
7.65 \\
7.39 \\
7.16 \\
6.83 \\
6.66 \\
6.25 \\
5.93 \\
5.76\end{array}$ & $\begin{array}{r}2,000 \\
1,820 \\
1,670 \\
1,550 \\
1,380 \\
1,300 \\
1,100 \\
935 \\
850\end{array}$ \\
\hline
\end{tabular}


(142) Naugatuck River near Thomaston, Conn.

Location.--Lat $41^{\circ} 42 \cdot 15^{\prime \prime}$, long $73^{\circ} 03^{\prime} 53^{\prime \prime}$, on right bank near downstream side of Twomlle Bridge, $250 \mathrm{ft}$ downstream from New York, New Haven \& Hartford Railroad bridge, 0.4 mile upstream from Leadmine Brook and 2 miles north of Thomaston, Litchfield County, and at mile 31. Datum of gage is $389.44 \mathrm{ft}$ above mean sea level, datum of 1929 (levels by Corps of Engineers).

Drainage area.--71.9 $\mathrm{sq} \mathrm{mi}$.

Gage-height record.--Water-stage recorder graph except for period 4 a.m. Aug. 19 to 3 p.m. Aug. 20 and Aug. 21 to 30. Graph for period $4 \mathrm{a} . \mathrm{m}$. Aug. 19 to $3 \mathrm{p} . \mathrm{m}$. Aug 20 was drawn on basis of floodmark and time of peak and for Aug. 21 to 30 on basis of shape of recession graph for former floods.

Discharge record.--Stage-discharge relation defined by current-meter measurements below 6,000 cf's and by slope-area determination of peak flow.

Maxima.--August 1955: Discharge, 41,600 ofs 5:30 a.m. Aug. 19 (gage height, 24.0 ft, from floodmarks). 1930 to July 1955: Discharge 10,200 cfs Dec. 31, 1948 (gage height, $12.03 \mathrm{ft}$ ).

Mean discharge, in cuible feet per second, August 1955

\begin{tabular}{r|r||r|r||r|r||r|r|r|r||r||r|}
\hline Day & Cfs & Day & Cfs & Day & Cfs & Day & Cfs & Daj & Cfs & Daj & Cfs \\
\hline 1 & 13 & 7 & 26 & 12 & 71 & 17 & 110 & 22 & 500 & 27 & 180 \\
2 & 20 & 8 & 31 & 13 & 433 & 18 & 2,160 & 23 & 350 & 170 \\
3 & 19 & 9 & 24 & 14 & 1,690 & 19 & 14,700 & 24 & 280 & 28 & 170 \\
4 & 21 & 10 & 23 & 15 & 442 & 20 & 2,320 & 25 & 220 & 30 & 145 \\
5 & 25 & 11 & 35 & 16 & 187 & 21 & 1,000 & 26 & 190 & 31 & 142 \\
6 & 42 & & & & & & & & & \\
\hline
\end{tabular}

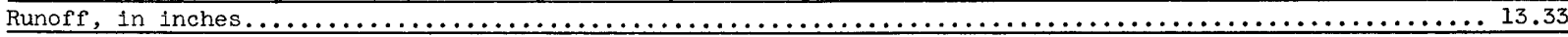

Gage helght, in feet, and discharge, in cubic feet per second, at indicated time

\begin{tabular}{|c|c|c|c|c|c|c|c|c|c|c|c|}
\hline Time & $\begin{array}{c}\text { Gage } \\
\text { height }\end{array}$ & $\begin{array}{l}\text { Dis- } \\
\text { charge }\end{array}$ & Time & $\begin{array}{l}\text { Gage } \\
\text { helght }\end{array}$ & $\begin{array}{l}\text { Dis- } \\
\text { charge }\end{array}$ & Time & $\begin{array}{c}\text { Gage } \\
\text { he1ght }\end{array}$ & $\begin{array}{l}\text { Dis- } \\
\text { charge }\end{array}$ & Time & $\begin{array}{c}\text { Gage } \\
\text { height }\end{array}$ & $\begin{array}{l}\text { Dis - } \\
\text { charge }\end{array}$ \\
\hline$\frac{\text { Aug. } 17}{12 \mathrm{p} \cdot \mathrm{m}}$ & 1.74 & 100 & Aug. $18--C o n$. & 7.54 & 4,450 & $\frac{\text { Aug. } 19--C o n}{3: 30-}$ & 16.24 & 19,600 & $\frac{\text { Aug. } 19--C}{6-\ldots-}$ & 10.2 & 7,950 \\
\hline & & & & 6.85 & 3,700 & $4-$ & 15.52 & 17,800 & $8-\cdots$ & 9.2 & 6,530 \\
\hline Aug. 18 & & & $7--$ & 6.13 & 3,000 & $4: 30-\ldots$ & 21.5 & 33,100 & $10-\cdots$ & 8.3 & 5,360 \\
\hline $6 \mathrm{a} \cdot \mathrm{m}$. & 1.70 & 93 & & 5.65 & 2,560 & $5-1-1-1-$ & 23.8 & 41,000 & $12 \ldots$ & 7.5 & 4,400 \\
\hline $7---$ & 1.95 & 144 & $9-\ldots$ & 5.32 & 2,270 & $5: 30 \ldots$ & 24.0 & 41,600 & & & \\
\hline $8---$ & 2.85 & 465 & $10 \cdots$ & 5.07 & 2,040 & $6-\ldots$ & 23.9 & 41,300 & Aug. 20 & & \\
\hline $9--$ & 4.20 & 1,310 & $11 \cdots$ & 5.65 & 2,560 & 7----- & 21.8 & 34,700 & $2 \mathrm{a} \cdot \mathrm{m}$ & 6.8 & 3,650 \\
\hline $10-\ldots$ & 6.17 & 3,030 & $12 \cdots \cdots$ & 7.21 & 4,080 & $8-\ldots$ & 18.9 & 26,200 & $4-\cdots$ & 6.3 & 3,150 \\
\hline $11=--$ & 6.45 & 3,300 & & & & $9-\cdots$ & 17.0 & 21,400 & $6-\cdots$ & 5.9 & 2,790 \\
\hline $12 \mathrm{m.-}$ & 6.63 & 3,480 & Aug. 19 & & & $10 \ldots$ & 15.6 & 18,000 & $9-\ldots$ & 5.5 & 2,430 \\
\hline $1 \mathrm{p} . \mathrm{m}$ & 6.82 & 3,670 & $1 \mathrm{a} \cdot \mathrm{m}$. & 8.80 & 5,990 & $11-0$ & 14.5 & 15,800 & $12 \mathrm{~m}, \ldots$ & 5.2 & 2,160 \\
\hline $2--$ & 7.17 & 4,040 & $2-\cdots-$ & 9.30 & 6,670 & $12 \mathrm{m.n}$ & 13.6 & 13,800 & 3 p.m. & 4.95 & 1,940 \\
\hline $3 \cdots-\cdots$ & 7.60 & 4,520 & $2: 30 \cdots$ & 10.00 & 7,650 & 2 p.m. & 12.1 & 11,100 & $6-\cdots$ & 4.63 & 1,650 \\
\hline $4----$ & 7.72 & 4,660 & & 14.00 & 14,600 & & 11.1 & 9,370 & $12-\ldots$ & 4.12 & 1,250 \\
\hline
\end{tabular}

(169) Rondout Creek near Lackawack, N. Y.

Location.--Lat $41^{\circ} 46^{\prime} 30^{\prime \prime}$, long $74^{\circ} 24^{\prime} 10^{\prime \prime}$,on left bank at lownstream side of highway bridge known as Wilburs Bridge, $2 \frac{1}{2}$ miles southeast of Lackawack, Ulster County, and 3 miles downstream from Merriman Dam (Roundout Reservolr). Datum of gage is $601.07 \mathrm{ft}$ above mean sea level (datum of New York C1ty Board of Water Supply).

Drainage area.--100 sq $\mathrm{m} 1$.

Gage-height record.--Water-stage recorder graph except for period 12:40 p.m. Aug. 1 to $12: 50$ p.m. Aug. 5, for which discharge was estimated on basis of weather records.

Discharge record. --Stage-discharge relation defined by current-meter measurements below 1,400 cfs and extended to peak stage by logarithmic plotting.

Maxima.--August 1955: Discharge 2,360 cfs 1:15 a.m. Aug. 19 (gage height, $5.76 \mathrm{ft}$ )

1906 to July 1955: Discharge 26,700 ofs Aug. 26, 1928 (slope-area determination by Board of Water Supply, city of New York).

Remarks.--Practicaliy entire flow above Merriman Dam (drainage area, $94.4 \mathrm{sq} \mathrm{ml}$ ) diverted for water supply of city of New York, subsequent to May 9, 1951.

Mean discharge, in cubic feet per second, August 1955

\begin{tabular}{r|r||r|r|r|r|r|r|r|r|r||r}
\hline Day & Cfs & Day & Cfs & Day & Cfs & Day & Cfs & Day & Cfs & Day & Cfs \\
\hline 1 & 18 & 7 & 19 & 12 & 20 & 17 & 22 & 22 & 43 & 27 & 28 \\
2 & 18 & 8 & 19 & 13 & 75 & 18 & 356 & 23 & 37 & 28 & 27 \\
3 & 18 & 9 & 19 & 14 & 33 & 19 & 561 & 24 & 33 & 29 & 25 \\
4 & 19 & 10 & 19 & 15 & 22 & 20 & 94 & 25 & 30 & 30 & 23 \\
5 & 19 & 11 & 19 & 16 & 22 & 21 & 57 & 26 & 29 & 31 & 24 \\
6 & 19 & & & & & &
\end{tabular}

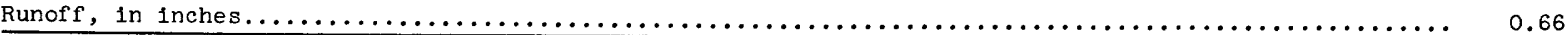


(163) Rondout Creek near Lackawack, N. Y.--Continued

Gage height, in feet, and discharge, in cublc feet per second, at indicated time

\begin{tabular}{|c|c|c|c|c|c|c|c|c|c|c|c|}
\hline Time & $\begin{array}{c}\text { Gage } \\
\text { height }\end{array}$ & $\begin{array}{l}\text { Dis- } \\
\text { charge }\end{array}$ & Time & $\begin{array}{c}\text { Gage } \\
\text { he1ght }\end{array}$ & $\begin{array}{l}\text { Dis - } \\
\text { charge }\end{array}$ & Time & $\begin{array}{c}\text { Gage } \\
\text { height }\end{array}$ & $\begin{array}{c}\text { Dis- } \\
\text { charge }\end{array}$ & Time & $\begin{array}{c}\text { Gage } \\
\text { he1ght }\end{array}$ & $\begin{array}{c}\text { Dis- } \\
\text { charge }\end{array}$ \\
\hline 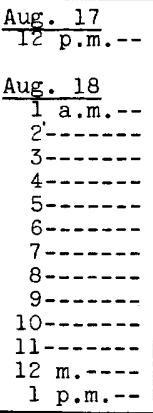 & $\begin{array}{r}.75 \\
.75 \\
.78 \\
.84 \\
.94 \\
1.10 \\
1.37 \\
1.56 \\
1.63 \\
1.96 \\
2.09 \\
2.25 \\
2.06\end{array}$ & $\begin{array}{r}25 \\
25 \\
26 \\
31 \\
38 \\
52 \\
81 \\
106 \\
115 \\
168 \\
194 \\
230 \\
188\end{array}$ & 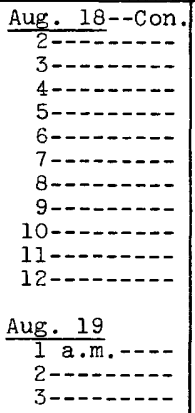 & $\begin{array}{l}1.90 \\
1.79 \\
1.70 \\
1.63 \\
1.92 \\
2.62 \\
3.48 \\
4.76 \\
5.06 \\
4.91 \\
5.26\end{array}$ & $\begin{array}{r}157 \\
138 \\
125 \\
115 \\
161 \\
326 \\
653 \\
1,430 \\
1,680 \\
1,550 \\
1,860 \\
\\
2,300 \\
2,080 \\
1,420\end{array}$ & 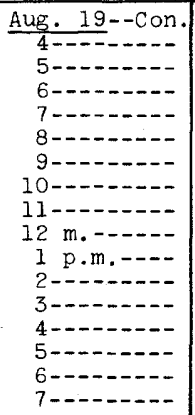 & $\begin{array}{l}4.02 \\
3.59 \\
3.33 \\
3.16 \\
3.01 \\
2.87 \\
2.74 \\
2.62 \\
2.50 \\
2.40 \\
2.31 \\
2.22 \\
2.15 \\
2.09 \\
2.02 \\
1.97\end{array}$ & $\begin{array}{l}934 \\
705 \\
585 \\
514 \\
456 \\
406 \\
363 \\
326 \\
292 \\
266 \\
244 \\
223 \\
207 \\
194 \\
180 \\
170\end{array}$ & 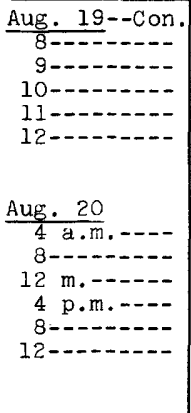 & $\begin{array}{l}1.65 \\
1.55 \\
1.45 \\
1.35 \\
1.29 \\
1.25\end{array}$ & $\begin{array}{l}161 \\
154 \\
147 \\
140 \\
134\end{array}$ \\
\hline
\end{tabular}

(175) Rondout Creek at Rosendale, N. Y.

Location.--Lat $41^{\circ} 50^{\prime} 35^{\prime \prime}$, long $74^{\circ} 05^{\prime} 10^{\prime \prime}$, on left bank $150 \mathrm{ft}$ upstream from upper highway bridge in Rosendale, Ulster County, and 3 miles upstream from Wallk1ll River. Datum of gage is $42.83 \mathrm{ft}$ above mean sea level, datum of 1929 , supplementary adjustment of 1943.

Drainage area. $--386 \mathrm{sq} \mathrm{mi}$

Gage-helght record.--Water-stage recorder graph, except for per1od 4 a.m., Aug. 19 to 4 p.m., Aug. 20 , for which graph was reconstructed on basis of high-water mark in gage house.

Discharge record.--Stage-discharge defined by current-meter measurements below 15,000 cfs and extended to peak stage on basis of contracted-opening determination.

Maxima.--August 1955: Discharge $30,900 \mathrm{cfs}$ about $6 \mathrm{a} . \mathrm{m}$. Aug. 19 (gage height, $23.93 \mathrm{ft}$, from high-water mark in gage house)

1901-03, 1906-19, 1926 to July 1955: Discharge, 27,300 cfs Aug. 27, 1928 (gage he1ght, 21.9 ft) from rating curve extended by logarithmic plotting.

Remarks.--Practically entire flow above Merriman Dam (drainage area, $94.4 \mathrm{sq} \mathrm{mi}$ ) diverted for water supply of city of New York, subsequent to May 9 , 1951.

\begin{tabular}{|c|c|c|c|c|c|c|c|c|c|c|c|}
\hline$\overline{\text { Day }}$ & Cfs & Day & $\mathrm{Cfs}$ & Day & Cis & Day & $\mathrm{cfs}^{\prime}$ & Day & Cfs & $\overline{D a y}$ & Cfs \\
\hline $\begin{array}{l}1 \\
2 \\
3 \\
4 \\
5 \\
6\end{array}$ & $\begin{array}{l}33 \\
58 \\
37 \\
30 \\
31 \\
33\end{array}$ & $\begin{array}{r}7 \\
8 \\
9 \\
10 \\
11\end{array}$ & $\begin{array}{l}36 \\
34 \\
42 \\
42 \\
34\end{array}$ & $\begin{array}{l}12 \\
13 \\
14 \\
15 \\
16\end{array}$ & $\begin{array}{r}47 \\
1,690 \\
1,800 \\
881 \\
371\end{array}$ & $\begin{array}{l}17 \\
18 \\
19 \\
20 \\
21\end{array}$ & $\begin{array}{r}286 \\
2,300 \\
17,900 \\
4,560 \\
2,170\end{array}$ & $\begin{array}{l}22 \\
23 \\
24 \\
25 \\
26\end{array}$ & $\begin{array}{r}1,320 \\
884 \\
716 \\
547 \\
485\end{array}$ & $\begin{array}{l}27 \\
28 \\
29 \\
30 \\
31\end{array}$ & $\begin{array}{l}401 \\
332 \\
283 \\
255 \\
261\end{array}$ \\
\hline
\end{tabular}

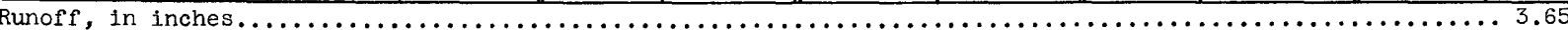

Gage he1ght, in feet, and discharge, in cublc feet per second, at indicated time

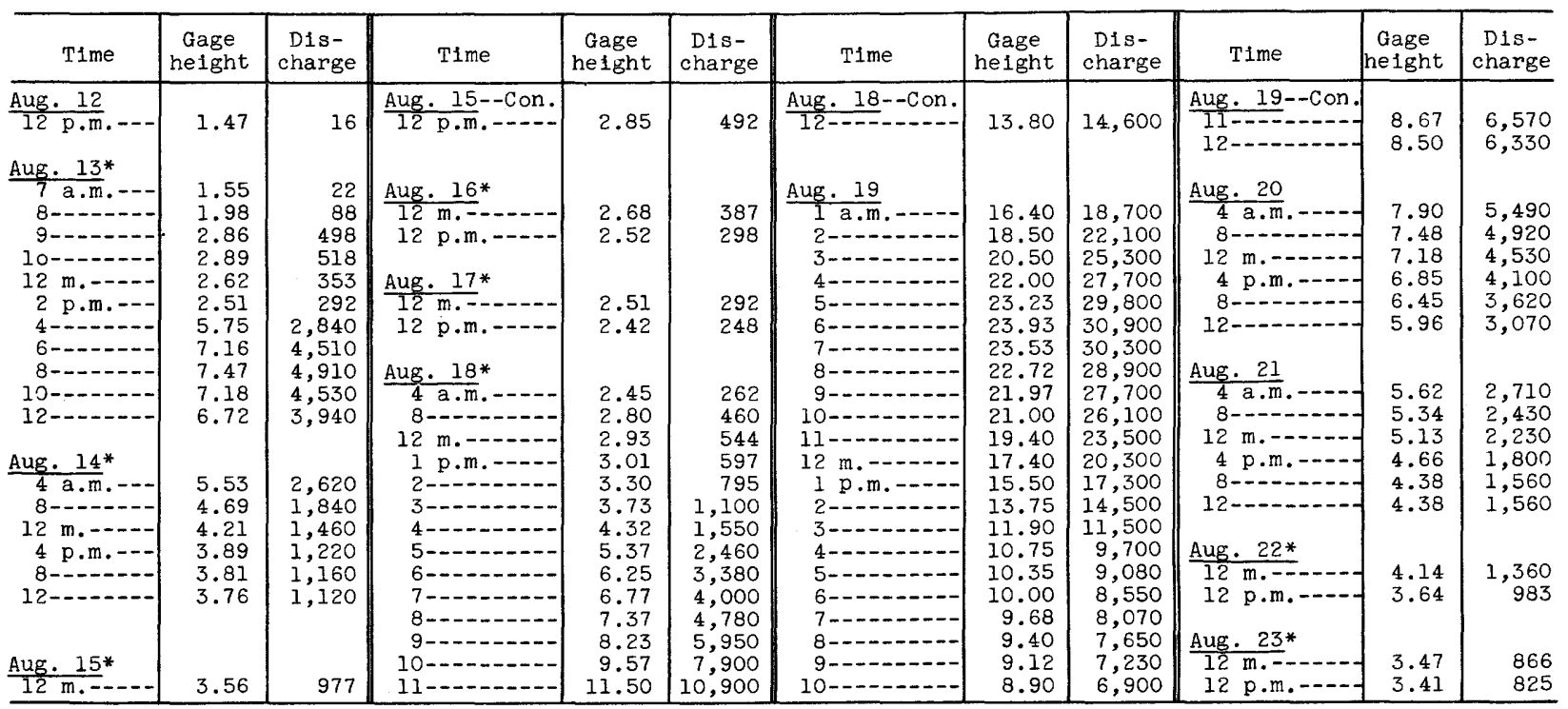


(184) Wallkill River at Gardiner, N. Y.

Location.--Lat $41^{\circ} 41^{\prime} 10^{\prime \prime}$, long $74^{\circ} \mathrm{Og} \mathrm{g}^{\prime} 55^{\prime \prime}$, on left bank $400 \mathrm{ft}$ upstream from highway bridge, 500 ft downstream from Shawangunk Kill, and three-quarters of a mile northwest of Gardiner, Ulster County. Datum of gage is $185.70 \mathrm{ft}$ above mean sea level, adjustment of 1912 .

Drainage area. --711 sq $\mathrm{m} 1$.

. discharge was estimated on basis of weather records.

ischarge record.--Stage-discharge relation defined by current-meter measurements.

Maxima -August 1955: Discharge 30,600 ofs 10:30 a.m. Aug. 19 (gage height, $19.77 \mathrm{ft}$ )

i924 to July 1955: Discharge 21 , 200 cfs June 1, 1952 (gage height, 14.88 ft); gage he1ght, 18.83 ft Mar. 7, 1945 (1ce Jam).

\begin{tabular}{|c|c|c|c|c|c|c|c|c|c|c|c|}
\hline Day & $\mathrm{Cfs}$ & Day & $\mathrm{Cfs}$ & Day & $\mathrm{Cfs}$ & Day & Cfs & Day & Cf s & Day & $\mathrm{Cfs}$ \\
\hline $\begin{array}{l}1 \\
2 \\
3 \\
4 \\
5 \\
6\end{array}$ & $\begin{array}{l}30 \\
30 \\
29 \\
28 \\
26 \\
25\end{array}$ & $\begin{array}{r}7 \\
8 \\
9 \\
10 \\
11\end{array}$ & $\begin{array}{l}29 \\
28 \\
21 \\
22 \\
56\end{array}$ & $\begin{array}{l}12 \\
13 \\
14 \\
15 \\
16\end{array}$ & $\begin{array}{l}119 \\
1,220 \\
2,800 \\
2,160 \\
2,150\end{array}$ & $\begin{array}{l}17 \\
18 \\
19 \\
20 \\
21\end{array}$ & $\begin{array}{r}2,020 \\
3,460 \\
25,200 \\
14,300 \\
10,400\end{array}$ & $\begin{array}{l}22 \\
23 \\
24 \\
25 \\
26\end{array}$ & $\begin{array}{l}9,910 \\
8,620 \\
6,680 \\
4,610 \\
2,870\end{array}$ & $\begin{array}{l}27 \\
28 \\
29 \\
30 \\
31\end{array}$ & $\begin{array}{r}2,000 \\
1,570 \\
1,320 \\
925 \\
798\end{array}$ \\
\hline
\end{tabular}

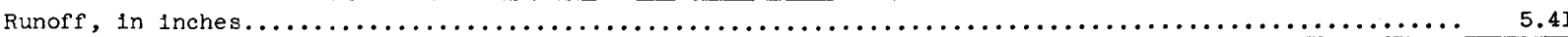

Gage height, in feet, and discharge, in cubic feet per second, at indicated time

\begin{tabular}{|c|c|c|c|c|c|c|c|c|c|c|c|}
\hline Time & $\begin{array}{l}\text { Gage } \\
\text { helght }\end{array}$ & $\begin{array}{l}\text { D1s- } \\
\text { harge }\end{array}$ & Time & $\begin{array}{l}\text { Gage } \\
\text { eight }\end{array}$ & $\begin{array}{l}\text { Dis- } \\
\text { charge }\end{array}$ & Time & & $\begin{array}{c}\text { Dis- } \\
\text { charge }\end{array}$ & Time & $\begin{array}{c}\text { Gage } \\
\text { height }\end{array}$ & $\begin{array}{c}\text { Dis- } \\
\text { charge }\end{array}$ \\
\hline 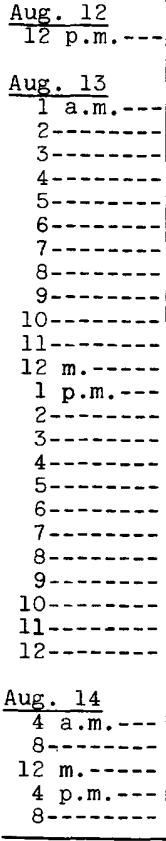 & $\begin{array}{l}2.71 \\
2.72 \\
2.75 \\
2.79 \\
2.84 \\
2.91 \\
2.96 \\
2.97 \\
3.01 \\
3.13 \\
3.29 \\
3.40 \\
3.57 \\
3.95 \\
4.25 \\
4.48 \\
4.80 \\
5.32 \\
5.74 \\
6.02 \\
6.17 \\
6.28 \\
6.32 \\
6.30\end{array}$ & $\begin{array}{r}197 \\
200 \\
212 \\
226 \\
246 \\
276 \\
299 \\
303 \\
322 \\
384 \\
476 \\
545 \\
662 \\
958 \\
1,220 \\
1,420 \\
1,720 \\
2,240 \\
2,390 \\
3,010 \\
3,190 \\
3,330 \\
3,370 \\
3,350\end{array}$ & 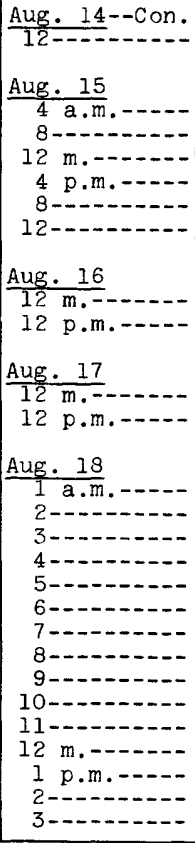 & $\begin{array}{l}5.00 \\
4.98 \\
4.97 \\
4.98 \\
4.99 \\
5.01 \\
5.05 \\
5.14 \\
5.25 \\
5.47 \\
5.60 \\
5.71 \\
5.81 \\
5.77 \\
5.73\end{array}$ & $\begin{array}{l}1,920 \\
1,900 \\
1,890 \\
1,900 \\
1,910 \\
1,930 \\
1,970 \\
2,060 \\
2,170 \\
2,400 \\
2,540 \\
2,660 \\
2,770 \\
2,730 \\
2,680\end{array}$ & 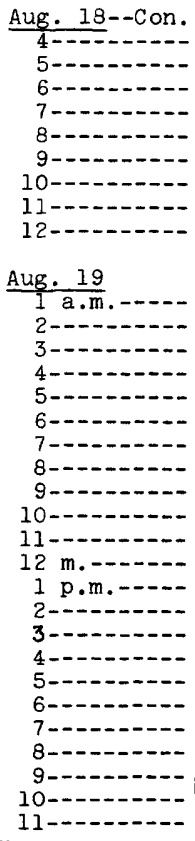 & $\begin{array}{l}5.75 \\
5.81 \\
6.03 \\
6.32 \\
6.81 \\
8.05 \\
10.20 \\
11.90 \\
13.20 \\
\\
14.30 \\
15.25 \\
16.76 \\
18.00 \\
18.60 \\
19.00 \\
19.26 \\
19.51 \\
19.68 \\
19.77 \\
19.77 \\
19.65 \\
19.45 \\
19.17 \\
18.85 \\
18.53 \\
18.15 \\
17.81 \\
17.45 \\
17.11 \\
16.85 \\
16.55 \\
16.28\end{array}$ & $\begin{array}{l}16,000 \\
18,100 \\
21,700 \\
25,000 \\
26,800 \\
28,000 \\
28,900 \\
29,700 \\
30,300 \\
30,600 \\
30,600 \\
30,200 \\
29,500 \\
28,600 \\
27,500 \\
26,500 \\
25,400 \\
24,500 \\
23,500 \\
22,600 \\
21,900 \\
21,200 \\
20,500\end{array}$ & 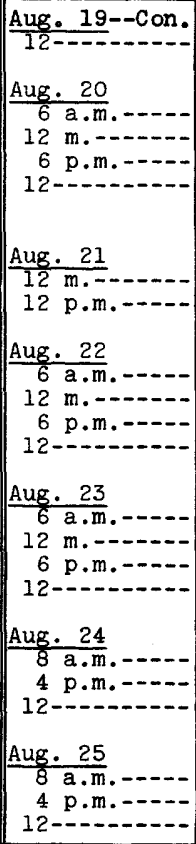 & $\begin{array}{l}14.59 \\
13.03 \\
12.00 \\
11.45\end{array}$ & $\begin{array}{r}10,300 \\
10,300 \\
\\
10,160 \\
9,970 \\
9,670 \\
9,350\end{array}$ \\
\hline
\end{tabular}


(185) Wappinger Creek near Wappingers Falls, N. Y.

Location. - Lat $41^{\circ} 39^{\prime} 0^{\prime \prime}$, long $73^{\circ} 52^{\prime} 20^{\prime \prime}$, on left bank $700 \mathrm{ft}$ downstream from Red Oak Mill dam and $4 \frac{1}{2} \mathrm{~m} 1 \mathrm{les}$ northeast of village of Wappingers Falls, Dutchess County. Altitude of gage is $120 \mathrm{ft}$ (from topographic map).

Drainage area.--182 sq mi.

Gage-height record.--Water-stage recorder graph, except for period $12 \mathrm{~m}$. to $6 \mathrm{p.m}$. Aug. 19, for wh1ch graph was reconstructed on basis of h1gh-water mark.

D1scharge record.--Stage-discharge relation defined by current-meter measurements below $3,800 \mathrm{cfs}$ and extended on basis of flow-over-dam determination at $18.02 \mathrm{ft}$, and contracted-opening and flow-over-road determination at $19.60 \mathrm{ft}$ made upstream at Manchester Bridge at Arlington, N. Y., with discharge of 18,000 cfs.

Maxima.--August 1955: Discharge, 18,600 cfs about $3 \mathrm{p} . \mathrm{m}$. Aug. ig (gage he1ght, $19.60 \mathrm{ft}$, from h1gh-water mark in gage house).

1928 to July 1955: D1scharge, 15,900 cf's Sept. 22, 1938 (gage height $18.02 \mathrm{ft}$, from floodmarks).

\begin{tabular}{r|r||r|r||r|r||r|r||r|r|r||r}
\hline Day & Cfs & Day & Cfs & Day & Cfs & Day & Cfs & Day & Cfs & Day & Cfs \\
\hline 1 & 14 & 7 & 12 & 12 & 12 & 17 & 95 & 22 & 1,400 & 27 & 473 \\
2 & 14 & 8 & 12 & 13 & 112 & 18 & 312 & 23 & 1,230 & 28 & 426 \\
3 & 13 & 9 & 11 & 14 & 410 & 19 & 10,500 & 24 & 916 & 29 & 365 \\
4 & 13 & 10 & 11 & 15 & 221 & 20 & 5,490 & 25 & 664 & 30 & 327 \\
5 & 12 & 11 & 11 & 16 & 137 & 21 & 2,290 & 26 & 551 & 31 \\
6 & 12 & & & & & & & & & \\
\hline
\end{tabular}

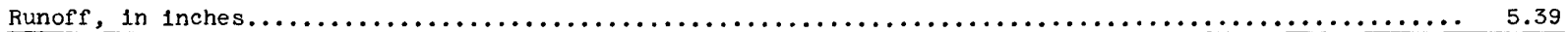

Gage height, in feet, and discharge, in cubic feet per second, at indicated time

\begin{tabular}{|c|c|c|c|c|c|c|c|c|c|c|c|}
\hline Time & $\begin{array}{c}\text { Gage } \\
\text { he1ght }\end{array}$ & $\begin{array}{l}\text { Dis- } \\
\text { charge }\end{array}$ & Time & $\begin{array}{c}\text { Gage } \\
\text { height }\end{array}$ & $\begin{array}{l}\text { Dis- } \\
\text { charge }\end{array}$ & Time & $\begin{array}{c}\text { Gage } \\
\text { he1ght }\end{array}$ & $\begin{array}{c}\text { Dis- } \\
\text { charge }\end{array}$ & Time & $\begin{array}{c}\text { Gage } \\
\text { height }\end{array}$ & $\begin{array}{c}\text { Dis- } \\
\text { charge }\end{array}$ \\
\hline Aug. 17 & & & Aug. 19- & & & Aug. $20^{*}-\mathrm{Con}$. & & & Aug. 22 & & \\
\hline & 3.14 & 83 & $10-1$. & $\begin{array}{l}14.30 \\
16.00\end{array}$ & $\begin{array}{l}10,700 \\
13,200\end{array}$ & $12 \mathrm{~m}$ & $\begin{array}{r}10.34 \\
9.94\end{array}$ & $\begin{array}{l}5,510 \\
5,030\end{array}$ & $12 \mathrm{~m}$ & & \\
\hline 2g. $18^{*}$ & & & $12 \mathrm{m.c-}$ & & 15,300 & 2 p.m. & & & 4 p.m. & & \\
\hline $4 \mathrm{a} \cdot \mathrm{m}$. & 3.14 & 83 & $1 \mathrm{p}$. & & & $4---$ & & & & & \\
\hline $8 \cdots$ & 3.24 & 99 & & & & $6-\cdots$ & 8. & 20 & $12-$ & & \\
\hline $12 \mathrm{~m}$ & 3.43 & 13 & $3-\cdots$ & & & $8---$ & 8. & 3,530 & & & $1,10+3$ \\
\hline & 3.76 & 226 & $4---$ & & & $10-\cdots$ & 8. & 3,380 & Aug. 23 & & \\
\hline & 4.76 & 660 & $5---$ & 18.70 & 17,200 & $12--\cdots$ & 8.2 & 3,190 & $4 \mathrm{a} \cdot \mathrm{m}$ & 5.61 & \\
\hline $12--$ & 5.95 & 1,350 & & 18.10 & 16,300 & & & & $8---$ & & \\
\hline & & & 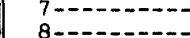 & & & g. 21 & & & $12 \mathrm{~m}$ & & \\
\hline g. 19 & & & $9-\ldots$ & & & 4 a.m. & & 2,790 & & & \\
\hline $1 \mathrm{a.m}$. & 6.26 & & $10--\ldots$ & & & $8-\cdots$ & & & $12---$ & & 1,09 \\
\hline & 6.69 & & $11-\ldots$ & & & $12 \mathrm{m.-.-}$ & & & & & \\
\hline & $\begin{array}{l}7.36 \\
7.91\end{array}$ & $\begin{array}{l}2,390 \\
2,880\end{array}$ & & 14.00 & 10,300 & 4 p.m.- & & 2,000 & Aug. 24 & & \\
\hline & 8.48 & 3,4 & Aug. $20^{*}$ & & & $12 \ldots$ & $\begin{array}{l}6.63 \\
6.40\end{array}$ & 1,650 & $\begin{array}{l}4 \mathrm{a} \cdot \mathrm{m} \cdot- \\
8\end{array}$ & & \\
\hline $6--$ & 9.50 & 4,5 & $2 \mathrm{a} \cdot \mathrm{m} .-$ & & & & & & $12 \mathrm{~m}, \ldots$ & & 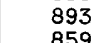 \\
\hline & & & $4--\cdots$ & & & 20 & & & 4 p.m.- - & & \\
\hline $\begin{array}{l}8- \\
9-\end{array}$ & $\begin{array}{l}11.50 \\
12.71\end{array}$ & $\begin{array}{l}6,910 \\
8,480\end{array}$ & 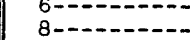 & $\begin{array}{l}11.43 \\
10.78\end{array}$ & $\begin{array}{l}6,8 \\
6,0\end{array}$ & $\frac{\text { Aug. } 22}{4 . \mathrm{m} .}$ & 6.27 & 1,560 & 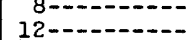 & $\begin{array}{l}5.14 \\
5.04\end{array}$ & 805 \\
\hline
\end{tabular}


(210) South Branch Raritan River at Stanton, N. J.

Location.--Lat $40^{\circ} 34^{\prime} 21^{\prime \prime}$, long $74^{\circ} 52^{\prime} 10^{\prime \prime}$, on right bank at downstream side of highway bridge near Stanton railroad station, Reading Township, Hunterdon County, half a mile upstream from Prescott Brook. Datum of zage is l25.01 $f t$ above mean sea level, datum of 1929 .

Drainage area. $--147 \mathrm{sq} \mathrm{mi}$

Gage-helght record,--Water-stage recorder graph.

Discharge record.--Stage-discharge relation defined by current-meter measurements below 6,500 cf s and extended to peak stage on basis of adjustment to station of contracted-opening determination of 22,900 cfs made 1.7 miles downstream and siope-area determination of 19,600 cfs made 0.4 mile downstream. Backwater from aquatic growth or debris, at times.

Maxima.--August 1955: Discharge, 18,000 cf's 2 a.m. Aug. 19 (gage height, $15.22 \mathrm{ft}$ ).

1903-6, 1919-July 1955: Discharge, 10,900 cf's Mar. 15, 1940, July 19, 1945 (gage height, 12.2 ft).

Mean discharge, in cubic feet per second, August 1955

\begin{tabular}{r|r||r|r||r|r||r|r|r||r||r||r|}
\hline Day & Cfs & Day & Cfs & Day & Cfs & Day & Cfs & Day & Cfs & Day & Cf's \\
\hline 1 & 72 & 7 & 83 & 12 & 740 & 17 & 300 & 22 & 880 & 27 & 310 \\
2 & 70 & 8 & 193 & 13 & 4,390 & 18 & 1,920 & 23 & 575 & 28 & 276 \\
3 & 65 & 9 & 87 & 14 & 1,390 & 19 & 8,060 & 24 & 453 \\
4 & 63 & 10 & 76 & 15 & 625 & 20 & 1,430 & 29 & 25 \\
5 & 65 & 11 & 119 & 16 & 346 & 21 & 924 & 39 & 26 & 338 \\
6 & 67 & & & & & 31 & 245 \\
\hline
\end{tabular}

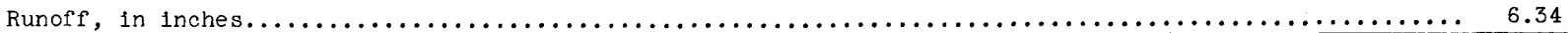

\begin{tabular}{|c|c|c|c|c|c|c|c|c|c|c|c|}
\hline Time & $\begin{array}{c}\text { Gage } \\
\text { height }\end{array}$ & $\begin{array}{l}\text { Dis- } \\
\text { charge }\end{array}$ & Time & $\begin{array}{c}\text { Gage } \\
\text { he1ght }\end{array}$ & $\begin{array}{c}\text { D1s- } \\
\text { charge }\end{array}$ & Time & $\begin{array}{c}\text { Gage } \\
\text { height }\end{array}$ & $\begin{array}{c}\text { Dis- } \\
\text { charge }\end{array}$ & Time & $\begin{array}{c}\text { Gage } \\
\text { he1ght }\end{array}$ & $\begin{array}{l}\text { Dis- } \\
\text { charge }\end{array}$ \\
\hline 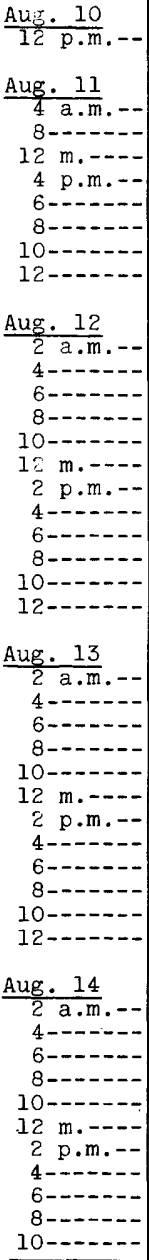 & $\begin{array}{r}4.82 \\
6.28 \\
8.15 \\
9.65 \\
10.29 \\
9.94 \\
9.72 \\
9.19 \\
8.48 \\
7.69 \\
7.03 \\
6.38\end{array}$ & $\begin{array}{l}1,210 \\
2,220 \\
4,140 \\
6,270 \\
7,330 \\
6,740 \\
6,380 \\
5,560 \\
4,560 \\
3,590 \\
2,890 \\
2,300\end{array}$ & 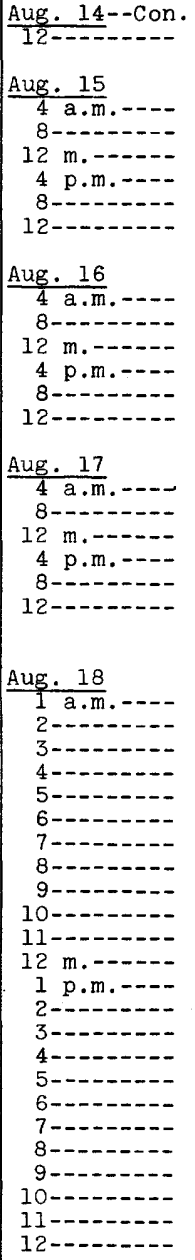 & $\begin{array}{r}3.50 \\
4.02 \\
4.73 \\
5.35 \\
5.41 \\
5.42 \\
5.37 \\
5.25 \\
5.08 \\
4.89 \\
4.72 \\
4.66 \\
4.64 \\
4.63 \\
4.60 \\
4.53 \\
4.50 \\
4.59 \\
5.15 \\
6.15 \\
7.50 \\
8.35 \\
9.85 \\
13.17\end{array}$ & $\begin{array}{r}460 \\
732 \\
1,160 \\
1,560 \\
1,600 \\
1,600 \\
1,570 \\
1,490 \\
1,380 \\
1,250 \\
1,150 \\
1,120 \\
1,100 \\
1,100 \\
1,080 \\
1,040 \\
1,020 \\
1,070 \\
1,420 \\
2,120 \\
3,380 \\
4,400 \\
6,590 \\
13,000\end{array}$ & 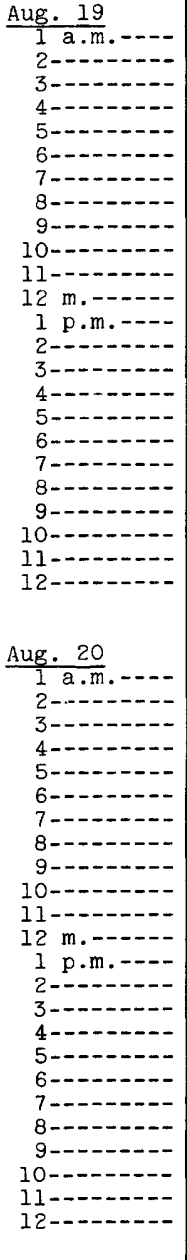 & $\begin{array}{r}14.53 \\
15.22 \\
14.84 \\
13.75 \\
12.75 \\
12.00 \\
11.49 \\
11.26 \\
11.14 \\
10.75 \\
10.37 \\
9.90 \\
9.50 \\
9.26 \\
9.04 \\
8.82 \\
8.60 \\
8.31 \\
8.00 \\
7.68 \\
7.32 \\
6.99 \\
6.66 \\
6.36\end{array}$ & $\begin{array}{r}16,300 \\
18,000 \\
17,100 \\
14,400 \\
12,200 \\
10,600 \\
9,580 \\
9,130 \\
8,910 \\
8,160 \\
7,480 \\
6,670 \\
6,030 \\
5,670 \\
5,340 \\
5,030 \\
4,720 \\
4,340 \\
3,960 \\
3,580 \\
3,180 \\
2,850 \\
2,540 \\
2,290\end{array}$ & 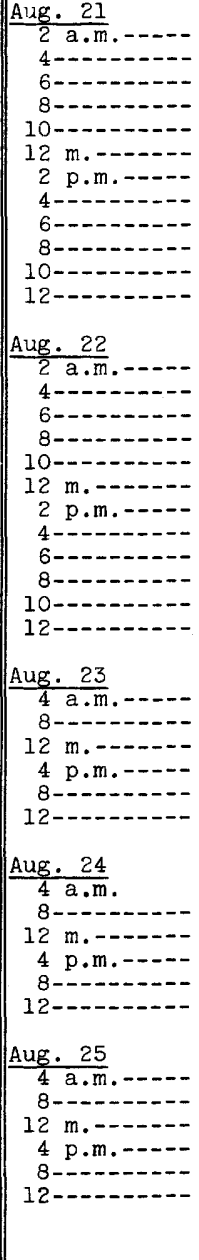 & $\begin{array}{l}3.82 \\
3.78 \\
3.75 \\
3.68 \\
3.62 \\
3.57 \\
\\
3.54 \\
3.52 \\
3.49 \\
3.45 \\
3.41 \\
3.39\end{array}$ & $\begin{array}{r}1,000 \\
972 \\
948 \\
930 \\
906 \\
876 \\
840 \\
804 \\
774 \\
786 \\
1,100 \\
1,270\end{array}$ \\
\hline
\end{tabular}


(227) Delaware River above Lackawaxen River, near Barryville, N. Y.

Location.--Lat $41^{\circ} 30 \cdot 30^{\prime \prime}$, long $74^{\circ} 59^{\prime} 15^{\prime \prime}$, on left bank 1.6 miles upstream from Lackawaxen River and 5.8 miles nort $n-$ west of Barryville, Sullivan County. 'Datum of gage is $600.22 \mathrm{ft}$ above mean sea level (datum of 1929 , New YorkPennsylvania supplementary adjustment of 1943).

Drainage area. $--2,023 \mathrm{sq} \mathrm{m}$.

Gage-height record.-Water-stage recorder graph except for period 9 p.m. Aug. 18 to 9 a.m. Aug. 22, for which graph was reconstructed on basis of high-water mark in gage house and outside-gage readings.

Discharge record. - Stage-discharge relation defined by current-meter measurements below 55,000 cfs and by slope-area determination at $23.19 \mathrm{ft}$.

Maxima.--August 1955: Discharge, $130,000 \mathrm{cfs}$, about 3:30 a.m. Aug. 19 (gage helght, 26.40 ft, from high-water mark in gage house).

1940 to July 1955: Discharge, 105,000 cfs, May 23, 1942 (gage height, $23.19 \mathrm{ft}$ ), from slope-area determination. Remarks.--Practically entire flow above Downsvilie Dam (drainage area, $371 \mathrm{sq}$ mi) diverted for water supply of cit. of New York, subsequent to Sept. 15, 1954.

Mean discharge, in cubic feet per second, August 1955

\begin{tabular}{c|c|c|c|c|c|c|c|c|c||c|c}
\hline Day & Cfs & Day & Cfs & Day & Cfs & Day & Cfs & Day & Cfs & Day & Cfs \\
\hline 1 & 532 & 7 & 566 & 12 & 808 & 17 & 1,930 & 22 & 9,480 & 27 & 2,910 \\
2 & 541 & 8 & 576 & 13 & 2,720 & 18 & 10,400 & 23 & 9,720 & 28 & 2,520 \\
3 & 516 & 9 & 605 & 14 & 6,100 & 19 & 85,000 & 24 & 6,750 & 29 & 2,200 \\
4 & 505 & 10 & 560 & 15 & 5,180 & 20 & 25,800 & 25 & 4,770 & 30 & 1,840 \\
5 & 500 & 11 & 565 & 16 & 2,920 & 21 & 12,700 & 26 & 3,600 & 31 & 2,140 \\
6 & 523 & & & & & & & & & & \\
\hline
\end{tabular}

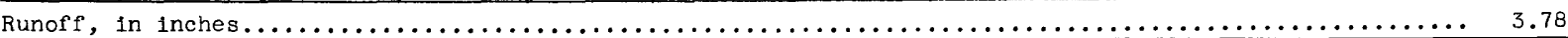

Gage height, in feet, and discharge, in cubic feet per second, at indicated time

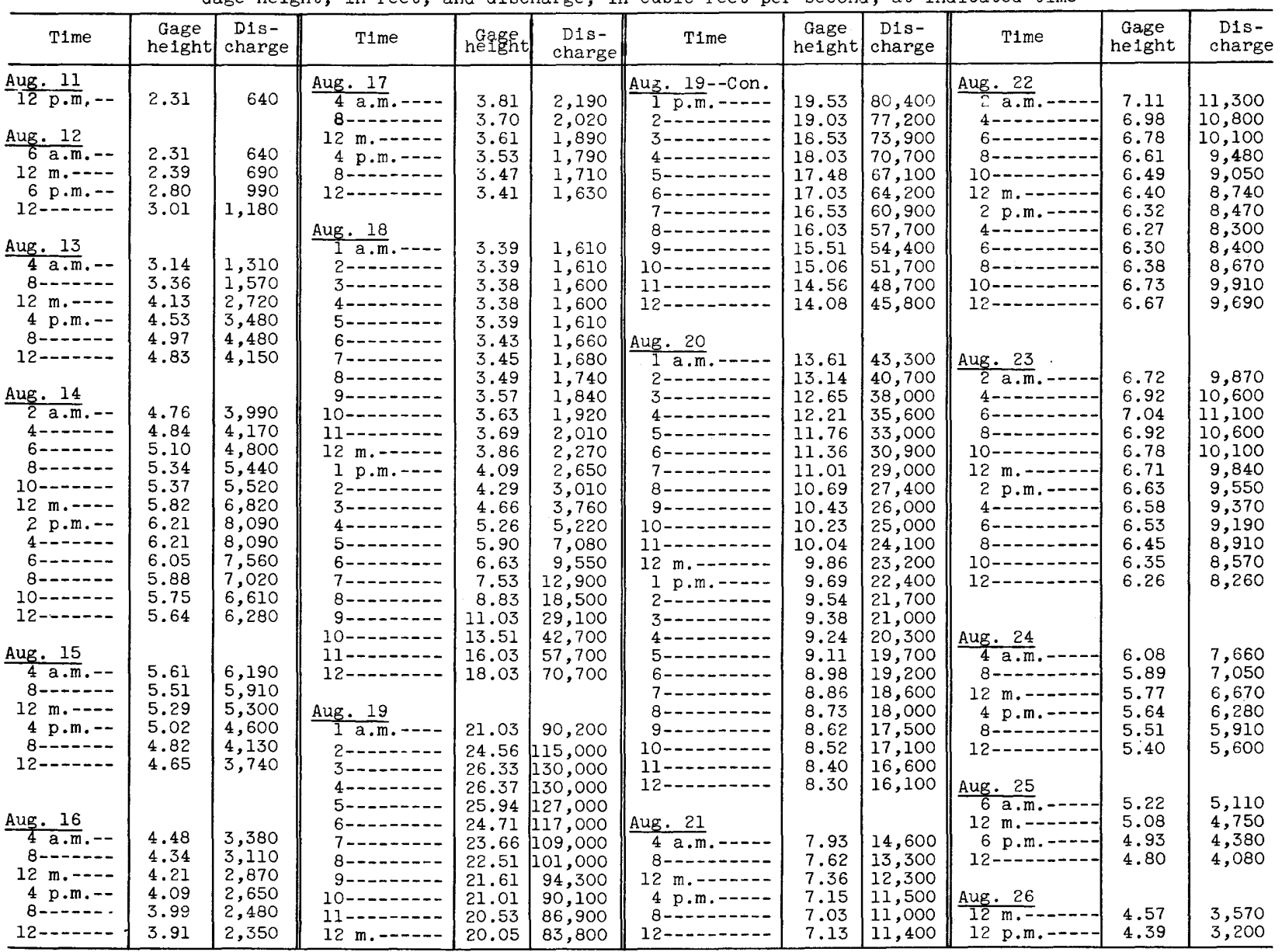


(231) Middle Creek near Hawley, Pa.

Location:--Lat $41^{\circ} 29^{\prime} 05^{\prime \prime}$, long $75^{\circ} 13^{\prime} 20^{\prime \prime}$, on downstream side of left span of highway bridge near center pler, 0.1 mile downstream from Red Shale Brook, 2 miles northwest of Hawley, Wayne County, and 2.5 miles upstream from mouth. Datum of gage is $1,017.73 \mathrm{ft}$ above mean sea leve1, unadjusted.

Drainage area. $--78.4 \mathrm{sq} \mathrm{mi}$.

Gage-helght record. - Wire-weight gage read twice dally and peak from crest-stage indicator.

Discharge record.--Stage-discharge relation defined by current-meter measurements below 3,500 cfs and extended above on basis of slope-area determinations at gage heights $13.22 \mathrm{ft}$ and $17.87 \mathrm{ft}$.

Maxima.--August 1955: Discharge, 12,000 cfs 1 a.m. Aug. 19 (gage height, $17.87 \mathrm{ft}$, from crest-stage indicator). 1944 to July 1955: Discharge, 6,970 cfs July 10, 1952 (gage height, 14.17 ft), from rating curve extended as explained above.

Flood of May 23, 1942 reached a stage of about $18 \mathrm{ft}$.

Mean discharge, in cubic feet per second, August 1955

\begin{tabular}{|c|c|c|c|c|c|c|c|c|c|c|c|}
\hline Day & Cfs & Day & Cfs & Day & Cfs & Day & $\mathrm{Cfs}$ & Day & Cfs & Day & $\mathrm{Cfs}$ \\
\hline $\begin{array}{l}1 \\
2 \\
3 \\
4 \\
5 \\
6\end{array}$ & $\begin{array}{l}3.1 \\
2.9 \\
2.8 \\
2.8 \\
2.7 \\
2.7\end{array}$ & $\begin{array}{r}7 \\
8 \\
9 \\
10 \\
11\end{array}$ & $\begin{array}{l}6.2 \\
4.9 \\
3.7 \\
3.1 \\
4.3\end{array}$ & $\begin{array}{l}12 \\
13 \\
14 \\
15 \\
16\end{array}$ & $\begin{array}{r}22 \\
453 \\
629 \\
498 \\
197\end{array}$ & $\begin{array}{l}17 \\
18 \\
19 \\
20 \\
21\end{array}$ & $\begin{array}{r}108 \\
2,420 \\
6,740 \\
1,220 \\
548\end{array}$ & $\begin{array}{l}22 \\
23 \\
24 \\
25 \\
26\end{array}$ & $\begin{array}{r}422 \\
419 \\
207 \\
133 \\
94\end{array}$ & $\begin{array}{l}27 \\
28 \\
29 \\
30 \\
31\end{array}$ & $\begin{array}{l}80 \\
84 \\
54 \\
48 \\
93\end{array}$ \\
\hline
\end{tabular}

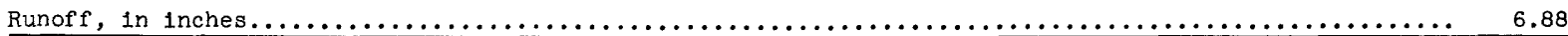

Gage height, in feet, and discharge, in cublc feet per second, at indicated time

\begin{tabular}{|c|c|c|c|c|c|c|c|c|c|c|c|}
\hline Time & $\begin{array}{c}\text { Gage } \\
\text { he1ght }\end{array}$ & $\begin{array}{l}\text { D1s- } \\
\text { charge }\end{array}$ & Time & $\begin{array}{c}\text { Gage } \\
\text { height }\end{array}$ & $\begin{array}{c}\text { Dis- } \\
\text { charge }\end{array}$ & TIme & $\begin{array}{c}\text { Gage } \\
\text { he1ght }\end{array}$ & $\begin{array}{c}\text { Dis- } \\
\text { charge }\end{array}$ & Time & $\begin{array}{c}\text { Gage } \\
\text { helght }\end{array}$ & $\begin{array}{l}\text { Dis- } \\
\text { charge }\end{array}$ \\
\hline 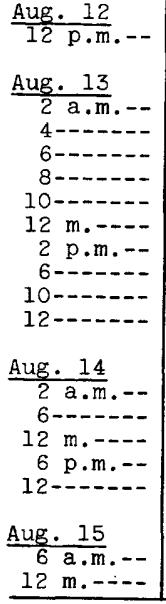 & $\begin{array}{l}2.78 \\
3.20 \\
3.74 \\
4.49 \\
5.34 \\
5.94 \\
5.92 \\
5.45 \\
5.07 \\
5.50\end{array}$ & $\begin{array}{r}59 \\
103 \\
181 \\
336 \\
571 \\
769 \\
762 \\
606 \\
491 \\
622\end{array}$ & 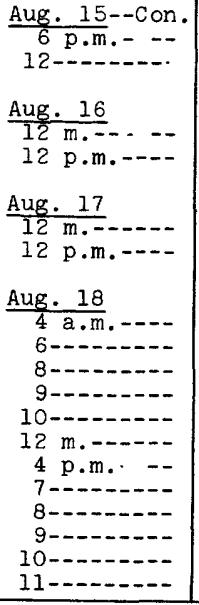 & $\begin{array}{r}3.23 \\
3.51 \\
4.20 \\
4.99 \\
5.55 \\
6.75 \\
8.00 \\
10.00 \\
13.00 \\
15.12 \\
16.17 \\
16.96 \\
\end{array}$ & $\begin{array}{r}186 \\
133 \\
\\
105 \\
88 \\
\\
108 \\
144 \\
269 \\
468 \\
638 \\
1,100 \\
1,730 \\
3,060 \\
5,790 \\
8,260 \\
9,620 \\
10,700 \\
\end{array}$ & 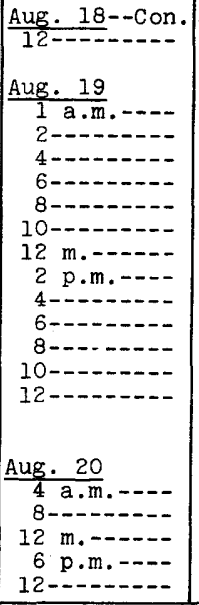 & $\begin{array}{r}17.87 \\
17.56 \\
16.46 \\
15.22 \\
14.13 \\
13.04 \\
11.96 \\
10.73 \\
9.68 \\
8.91 \\
8.18 \\
7.53 \\
6.91\end{array}$ & $\begin{array}{r}12,000 \\
11,600 \\
10,400 \\
9,320 \\
8,340 \\
7,360 \\
6,400 \\
5,410 \\
4,570 \\
3,960 \\
3,410 \\
2,950 \\
2,520\end{array}$ & 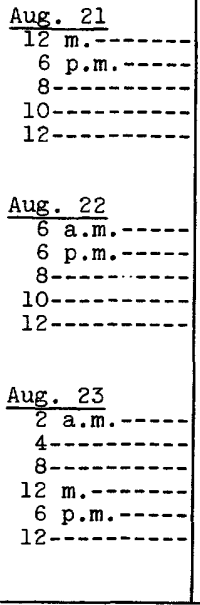 & $\begin{array}{l}3.29 \\
3.11 \\
2.65 \\
2.36 \\
2.11 \\
1.95\end{array}$ & $\begin{array}{l}675 \\
610 \\
456 \\
371 \\
304 \\
264\end{array}$ \\
\hline
\end{tabular}


(232) Lackawaxen River at Hawley, Pa.

Location.--Lat $41^{\circ} 28^{\prime} 30^{\prime \prime}$, long $75^{\circ} 10^{\prime} 5^{\prime \prime}$, on left bank at Hawley, wayne County, 300 ft downstream from Wallenpaupack Creek and 1,000 ft downstream from highway bridge. Datum of gage is $869.00 \mathrm{ft}$ above mean sea level, datum of 1929 , supplementary adjustment of 1943 .

Drainage area.--290 sq $\mathrm{mi}$ (excludes that of wallenpaupack Creek, which is $228 \mathrm{sq} \mathrm{mi}$, and flow from which 1s diverted around station to hydroelectric plant downstream.)

Gage-height record.--Water-stage recorder graph to $11 \mathrm{p} . \mathrm{m}$. Aug. 18 at which time recording gage was destroyed. Record from $11 \mathrm{p.m}$. Aug. 18 to $6 \mathrm{a} . \mathrm{m}$. Aug. 23 based on graph of once-daily gage readings at biridge $1,000 \mathrm{ft}$ upstream equated to recording gage site and floodmark of peak stage. Record from 6 a.m. Aug. 23 to Aug. 31 based on graph ot once or twice-daily readings of inclined staff gage di recording gage site.

Discharge record.--Stage-discharge relation defined by current-meter measurements below $12,000 \mathrm{cfs}$ and by slopearea determination at gage he1ght $20.1 \mathrm{ft}$

Maxima.--August 1955: Discharge, 51,900 cfs about $2 \mathrm{a.m}$. Aug. 19 (gage helght, $20.6 \mathrm{ft}$, from floodmark).

1908-19, 1938 to July 1955: Discharge, 50,000 cfs May 23, 1942 (gage height, $20.1 \mathrm{ft}$, from floodmark).

Flood of March 1936 reached a stage of $13.9 \mathrm{ft}$, from floodmark (discharge, 27,600 cfs).

Remarks.--At 11 p.m., August 18, the elevation of the water surface in Lake wallenpaupack was $1,182.7 \mathrm{ft}$ and $\mathrm{rising}$. Spill over the top of the roller gates began at 7:15 a.m. Aug. 19 on the basis of information furnished by Pennsylvania Power and Light Co. The peak stage and peak discharge of the Lackawaxen River at Hawley, which occurred at about $2 \mathrm{a} . \mathrm{m}$. August 19 , were not affected by spill over the gates. Daily discharges for the period August 19-31 include spill over the gates which is listed below, in cfs:

\begin{tabular}{c|c||c|c||c|c||c|c}
\hline Day & Discharge & Day & Discharge & Day & D1scharge & Day & Discharge \\
\hline 19 & 1,280 & 23 & 2,160 & 26 & 1,140 & 29 & 639 \\
20 & 2,900 & 24 & 1,710 & 27 & 944 & 30 & 480 \\
21 & 2,900 & 25 & 1,380 & 28 & 789 & 31 & 369 \\
22 & 2,650 & & & & \\
\hline
\end{tabular}

Mean discharge, in cubic feet per second, August 1955

\begin{tabular}{|c|c|c|c|c|c|c|c|c|c|c|c|}
\hline Day & Cfs & Day & Cfs & Day & Cfs & Day & Cfs & Day & Cfs & Day & Cfs \\
\hline $\begin{array}{l}1 \\
2 \\
3 \\
4 \\
5 \\
6\end{array}$ & $\begin{array}{l}22 \\
21 \\
20 \\
19 \\
19 \\
23\end{array}$ & $\begin{array}{r}7 \\
8 \\
9 \\
10 \\
11\end{array}$ & $\begin{array}{l}46 \\
32 \\
29 \\
24 \\
34\end{array}$ & $\begin{array}{l}12 \\
13 \\
14 \\
15 \\
16\end{array}$ & $\begin{array}{r}133 \\
2,390 \\
2,080 \\
1,400 \\
692\end{array}$ & $\begin{array}{l}17 \\
18 \\
19 \\
20 \\
21\end{array}$ & $\begin{array}{r}413 \\
10,800 \\
26,800 \\
6,810 \\
4,820\end{array}$ & $\begin{array}{l}22 \\
23 \\
24 \\
25 \\
26\end{array}$ & $\begin{array}{l}4,170 \\
4,240 \\
2,730 \\
2,060 \\
1,610\end{array}$ & $\begin{array}{l}27 \\
28 \\
29 \\
30 \\
31\end{array}$ & $\begin{array}{r}1,420 \\
1,310 \\
1,180 \\
862 \\
825\end{array}$ \\
\hline
\end{tabular}

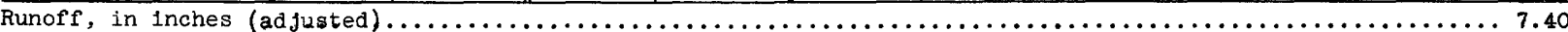

Gage height, in feet, and discharge, in cublc feet per second, at indicated time

\begin{tabular}{|c|c|c|c|c|c|c|c|c|c|c|c|}
\hline Time & $\begin{array}{c}\text { Gage } \\
\text { height }\end{array}$ & $\begin{array}{c}\text { D1s- } \\
\text { charge }\end{array}$ & TIme & $\begin{array}{c}\text { Gage } \\
\text { height }\end{array}$ & $\begin{array}{c}\text { Dis- } \\
\text { charge }\end{array}$ & Time & $\begin{array}{c}\text { Gage } \\
\text { helght }\end{array}$ & $\begin{array}{c}\text { Dis- } \\
\text { charge }\end{array}$ & Time & $\begin{array}{c}\text { Gage } \\
\text { he1ght }\end{array}$ & $\begin{array}{c}\text { Dis- } \\
\text { charge }\end{array}$ \\
\hline 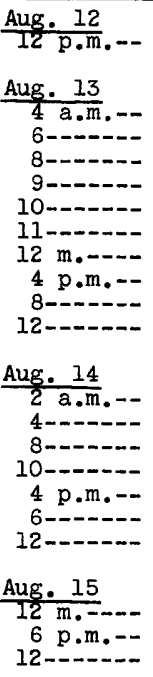 & $\begin{array}{l}2.13 \\
2.47 \\
3.00 \\
3.40 \\
4.35 \\
5.35 \\
5.88 \\
5.85 \\
5.28 \\
4.72\end{array}$ & $\begin{array}{r}217 \\
370 \\
675 \\
965 \\
1,960 \\
3,560 \\
4,580 \\
4,520 \\
3,430 \\
2,490\end{array}$ & 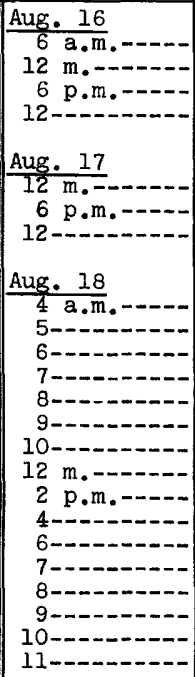 & $\begin{array}{r}2.54 \\
2.74 \\
3.00 \\
3.38 \\
5.20 \\
7.05 \\
7.50 \\
8.08 \\
8.40 \\
8.52 \\
8.87 \\
9.33 \\
10.10 \\
12.20 \\
14.15 \\
17.00\end{array}$ & $\begin{array}{r}404 \\
514 \\
675 \\
949 \\
3,290 \\
7,590 \\
8,850 \\
10,500 \\
11,400 \\
11,800 \\
12,800 \\
14,200 \\
16,500 \\
22,900 \\
29,200 \\
38,900\end{array}$ & 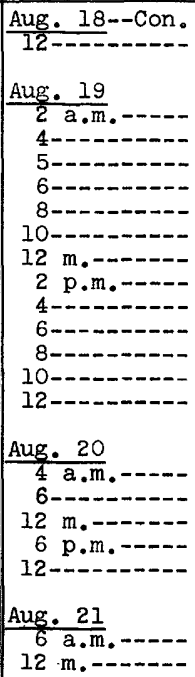 & $\begin{array}{r}20.60 \\
19.50 \\
18.37 \\
17.20 \\
15.35 \\
13.46 \\
12.00 \\
10.85 \\
9.87 \\
9.15 \\
8.63 \\
8.20 \\
7.80\end{array}$ & $\begin{array}{r}51,900 \\
47,800 \\
43,700 \\
39,600 \\
33,200 \\
27,000 \\
22,300 \\
18,800 \\
15,800 \\
13,600 \\
12,100 \\
10,900 \\
9,710\end{array}$ & 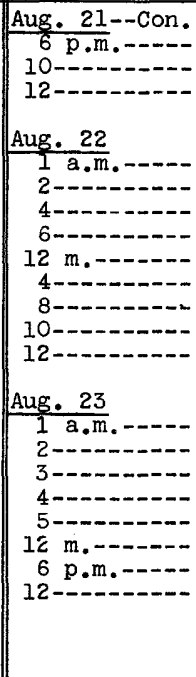 & $\begin{array}{l}6.43 \\
6.48 \\
5.82 \\
5.50 \\
5.27 \\
5.25 \\
5.50 \\
5.98 \\
6.68\end{array}$ & $\begin{array}{l}5,900 \\
6,030 \\
4,450 \\
3,830 \\
3,420 \\
3,380 \\
3,830 \\
4,800 \\
6,570 \\
\\
7,450 \\
7,590 \\
7,030 \\
5,310 \\
4,410 \\
3,780 \\
3,310 \\
3,050\end{array}$ \\
\hline
\end{tabular}


(242) Delaware River at Port Jervis, N. Y.

Location.--Lat $41^{\circ} 22^{\prime} 20^{\prime \prime}$, long $74^{\circ} 41^{\prime} 50^{\prime \prime}$, on right bank $250 \mathrm{ft}$ downstream from highway bridge on U. S. Highways 6 and 209 at Port Jervis, Orange County, $1 \frac{1}{2}$ miles upstream from Neversink River, and 6.5 miles downstream from Mongaup River. Datum of gage is $415.35 \mathrm{ft}$ above mean sea level, datum of 1929 .

Drainage area. $--3,076 \mathrm{sq} \mathrm{mi}$.

Gage-height record.--Water-stage recorder graph except for periods 3-8 p.m. Aug. 13 (graph reconstructed on basis of recorded range in stage) and 4-8 a.m. Aug 19 (graph reconstructed on basis of high-water mark in gage house). Discharge record.--Stage-discharge defined by current-meter measurements below 89 , $000 \mathrm{cfs}$ and extended to peak stage on basis of slope-area determinations at $14.95 \mathrm{ft}$ and $23.91 \mathrm{ft}$.

Maxima.--August 1955: Discharge, 233,000 cfs, about 5:30 a.m. Aug. 19 (gage height, 23.91 ft, from high-water mark in gage house)

1904 to July 1955: D1scharge 140,000 cfs, May 23, 1942 (gage height, 17.76 ft), from rating curve extended above $89,000 \mathrm{cfs}$ by logarithmic plotting on basis of slope-area determination.

oct. 10, 1903: Discharge, 205,000 cfs (gage he1ght, $23.1 \mathrm{ft}$, reported by United States Weather Bureau), from rating curve extended above 70,000 cfs by logarithmic plotting and velocity-area studies; maximum stage known, $25.5 \mathrm{ft}$ Mar. 8, 1904 (1ce jam).

Remarks.--Practically entire flow above Downsville Dam (drainage area, 371 sq mi) diverted for water supply of city of New York, subsequent to Sept. 15, 1954. Flow regulated by Lake Wallenpaupack and by Toronto, Cliff Lake, and Swinging Bridge reservoirs (combined capac1ty, $12,300,000,000 \mathrm{cu} \mathrm{ft}$ ).

Mean discharge, in cubic feet per second, August 1955

\begin{tabular}{|c|c|c|c|c|c|c|c|c|c|c|c|}
\hline Day & Cfs & Day & $\mathrm{Cf}^{\prime} \mathrm{s}$ & Day & $\operatorname{Cfs}$ & Day & Cf's & Day & $\mathrm{Cfs}$ & Day & Cfs \\
\hline $\begin{array}{l}1 \\
2 \\
3 \\
4 \\
5\end{array}$ & $\begin{array}{r}762 \\
1,350 \\
1,430 \\
1,300 \\
1,400\end{array}$ & $\begin{array}{r}7 \\
8 \\
9 \\
10 \\
11\end{array}$ & $\begin{array}{l}810 \\
850 \\
810 \\
807 \\
945\end{array}$ & $\begin{array}{l}12 \\
13 \\
14 \\
15 \\
16\end{array}$ & $\begin{array}{r}1,450 \\
5,640 \\
10,600 \\
9,170 \\
5,530\end{array}$ & $\begin{array}{l}17 \\
18 \\
19 \\
20 \\
21\end{array}$ & $\begin{array}{r}4,170 \\
13,700 \\
163,000 \\
49,900 \\
23,400\end{array}$ & $\begin{array}{l}22 \\
23 \\
24 \\
25 \\
26\end{array}$ & $\begin{array}{r}17,400 \\
17,100 \\
12,700 \\
9,140 \\
7,170\end{array}$ & $\begin{array}{l}27 \\
28 \\
29 \\
30 \\
31\end{array}$ & $\begin{array}{l}5,770 \\
4,870 \\
4,570 \\
4,320 \\
4,960\end{array}$ \\
\hline
\end{tabular}

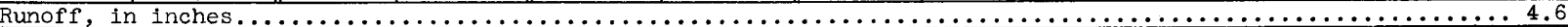

Gage height, in feet, and ajscharge, in cubic feet per second, at indicated time

\begin{tabular}{|c|c|c|c|c|c|c|c|c|c|c|c|}
\hline Tim & $\begin{array}{l}\text { tage } \\
\text { eight }\end{array}$ & $\begin{array}{l}\text { D1s- } \\
\text { harge }\end{array}$ & Time & & & Time & & $\begin{array}{l}\text { Dis- } \\
\text { charge }\end{array}$ & Time & & $\begin{array}{l}\text { Dis- } \\
\text { charge }\end{array}$ \\
\hline 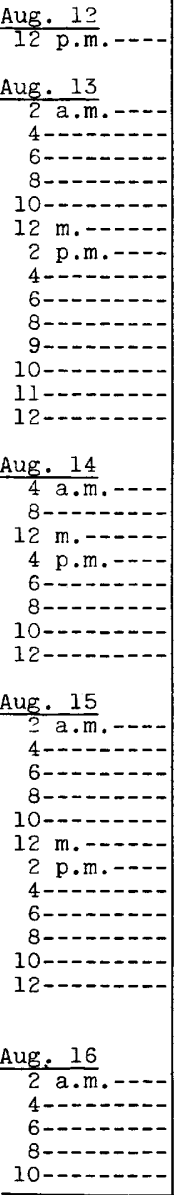 & $\begin{array}{l}1.98 \\
2.13 \\
2.34 \\
2.64 \\
2.94 \\
3.47 \\
4.03 \\
4.35 \\
4.61 \\
5.20 \\
5.25 \\
5.29 \\
5.33 \\
5.31\end{array}$ & $\begin{array}{r}1,230 \\
1,450 \\
1,810 \\
2,410 \\
3,110 \\
4,570 \\
6,420 \\
7,650 \\
8,740 \\
11,500 \\
11,800 \\
12,000 \\
12,200 \\
12,000\end{array}$ & 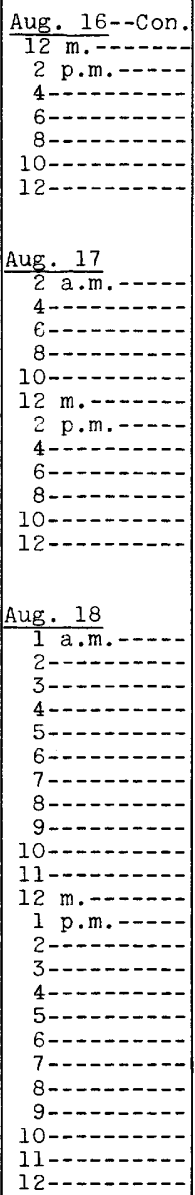 & $\begin{array}{r}3.04 \\
2.97 \\
2.92 \\
2.93 \\
3.00 \\
3.05 \\
3.10 \\
3.19 \\
3.27 \\
3.35 \\
3.39 \\
3.41 \\
3.41 \\
3.42 \\
3.46 \\
3.51 \\
4.35 \\
5.89 \\
6.50 \\
7.08 \\
8.15 \\
10.27 \\
12.60 \\
16.14\end{array}$ & $\begin{array}{r}3,360 \\
3,180 \\
3,060 \\
3,080 \\
3,260 \\
3,390 \\
3,520 \\
3,760 \\
3,990 \\
4,220 \\
4,330 \\
4,390 \\
4,390 \\
4,420 \\
4,540 \\
4,690 \\
7,650 \\
15,000 \\
18,300 \\
21,900 \\
29,500 \\
47,000 \\
70,500 \\
112,000\end{array}$ & 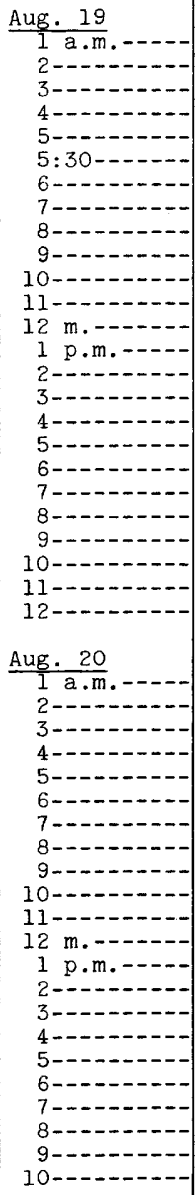 & $\begin{array}{l}18.86 \\
20.69 \\
22.40 \\
23.30 \\
23.88 \\
23.91 \\
23.80 \\
23.40 \\
22.93 \\
22.50 \\
22.00 \\
21.47 \\
20.65 \\
19.93 \\
19.21 \\
18.62 \\
18.00 \\
17.45 \\
16.90 \\
16.31 \\
15.73 \\
15.22 \\
14.64 \\
14.22 \\
13.75 \\
\end{array}$ & $\begin{array}{r}151,000 \\
179,000 \\
207,000 \\
223,000 \\
233,000 \\
233,000 \\
231,000 \\
224,000 \\
216,000 \\
209,000 \\
200,000 \\
192,000 \\
178,000 \\
167,000 \\
156,000 \\
147,000 \\
138,000 \\
130,000 \\
123,000 \\
115,000 \\
107,000 \\
101,000 \\
93,600 \\
88,500 \\
83,200\end{array}$ & 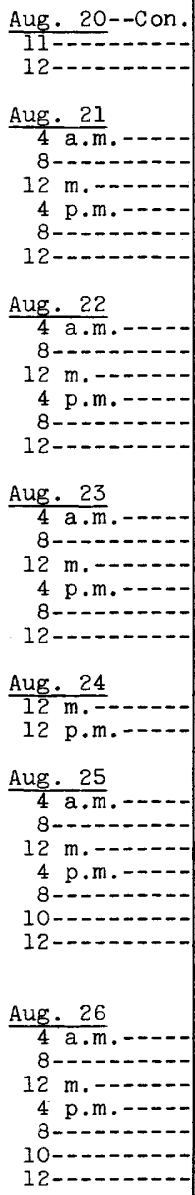 & $\begin{array}{l}4.92 \\
4.77 \\
4.70 \\
4.61 \\
4.40 \\
4.53 \\
4.39\end{array}$ & $\begin{array}{l}17,100 \\
18,400 \\
18,200 \\
16,800 \\
16,000 \\
15,100\end{array}$ \\
\hline
\end{tabular}


FLOOD DATA

(243) Neversink River at Oakland Valley, N. Y.

Location.--Lat $41^{\circ} 29145^{\prime \prime}$, long $74^{\circ} 38^{\prime} 45^{\prime \prime}$, on right bank 250 ft downstream from highway bridge known as Paradise Bridge, three-quarters of a mile downstream from Oakland Valley, Sullivan County, and $3 \frac{1}{2}$ miles northwest of cuddebackville. Datum of gage is $332.00 \mathrm{ft}$ above mean sea levei, adjustment of 1912

Drainage area. --222 $: \mathrm{a} \mathrm{m}$.

Gage-helght recordi.--Water-stage recorder graph.

Discharge record.--Stage-discharge relation defined by current-meter measurements below 7,800 cfs and extended to peak stage on basis of slope-area determination at $12.62 \mathrm{ft}$.

Maxima.--August 1955: Discharge, 23,800 cfs, 2:30 a.m. Aug. 19 (gage he1ght, $12.74 \mathrm{ft}$ ).

1928 to July 1955: Discharge, 23,300 cfs, Nov. 26, 1950 (gage height 12.62 ft), on basis of slope-area determination.

Remarks.--Practically entire flow above Neversink Dam (drainage area, $91.8 \mathrm{sq} \mathrm{mi}$ ) diverted for water supply to city of Nevi York, subsequent to June 2, 1953.

Mean d1scharge, in cubic feet per second, August 1955

\begin{tabular}{r|r||r|r||r|r||r|r||r|r|r|r}
\hline Day & Cfs & Day & Cfs & Day & Cf's & Day & Cf's & Day & Cfs & Day & Cfs \\
\hline 1 & 97 & 7 & 116 & 12 & 146 & 17 & 220 & 22 & 700 & 27 & 258 \\
2 & 101 & 9 & 106 & 13 & 1,540 & 18 & 2,510 & 23 & 847 & 28 & 222 \\
3 & 97 & 9 & 101 & 14 & 1,120 & 19 & 11,000 & 24 & 544 & 29 & 192 \\
4 & 96 & 10 & 105 & 15 & 614 & 20 & 2,240 & 25 & 380 & 30 & 179 \\
5 & 95 & 11 & 114 & 16 & 324 & 21 & 1,080 & 26 & 296 & 31 & 221 \\
6 & 96 & & & & & & & & & &
\end{tabular}

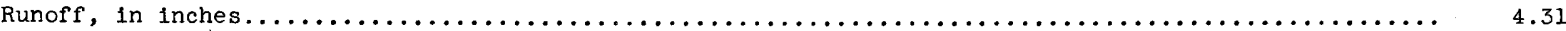

Gage height, in feet, and discharge, in cubic feet per second, at indicated time

\begin{tabular}{|c|c|c|c|c|c|c|c|c|c|c|c|}
\hline Time & $\begin{array}{c}\text { Gage } \\
\text { helght }\end{array}$ & $\begin{array}{l}\text { Dis- } \\
\text { charge }\end{array}$ & TIme & $\begin{array}{c}\text { Gage } \\
\text { height }\end{array}$ & $\begin{array}{l}\text { Dis- } \\
\text { charge }\end{array}$ & Time & $\begin{array}{c}\text { Gage } \\
\text { height }\end{array}$ & $\begin{array}{c}\text { Dis- } \\
\text { charge }\end{array}$ & Time & $\begin{array}{c}\text { Gage } \\
\text { height }\end{array}$ & $\begin{array}{l}\text { Dis- } \\
\text { charge }\end{array}$ \\
\hline 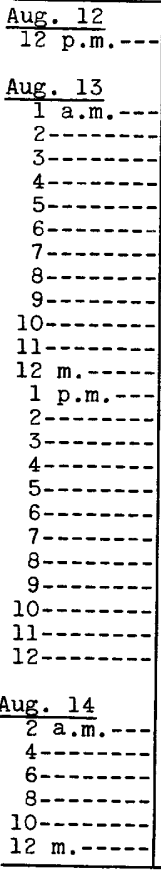 & $\begin{array}{l}2.01 \\
2.07 \\
2.11 \\
2.13 \\
2.21 \\
2.36 \\
2.60 \\
2.90 \\
3.28 \\
3.81 \\
4.11 \\
4.39 \\
4.82 \\
5.15 \\
5.22 \\
5.57 \\
5.70 \\
5.54 \\
5.25 \\
5.02 \\
4.82 \\
4.65 \\
4.50 \\
4.40\end{array}$ & $\begin{array}{r}186 \\
205 \\
218 \\
225 \\
253 \\
308 \\
411 \\
561 \\
795 \\
1,200 \\
1,460 \\
1,740 \\
2,200 \\
2,600 \\
2,700 \\
3,170 \\
3,350 \\
3,130 \\
2,740 \\
2,440 \\
2,200 \\
2,020 \\
1,850 \\
1,750\end{array}$ & 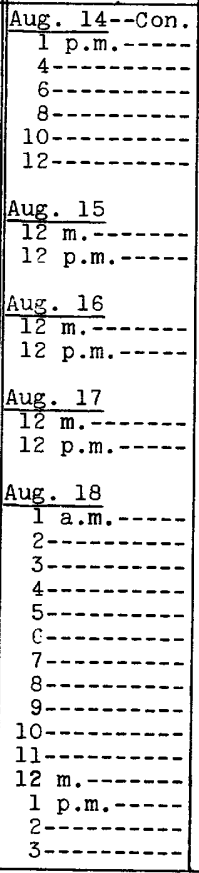 & $\begin{array}{l}1.97 \\
1.98 \\
2.06 \\
2.22 \\
2.42 \\
2.77 \\
3.02 \\
3.10 \\
3.15 \\
3.18 \\
3.24 \\
3.38 \\
3.59 \\
3.68 \\
3.73\end{array}$ & $\begin{array}{r}174 \\
177 \\
202 \\
256 \\
332 \\
493 \\
629 \\
677 \\
709 \\
728 \\
768 \\
865 \\
1,020 \\
1,090 \\
1,130\end{array}$ & 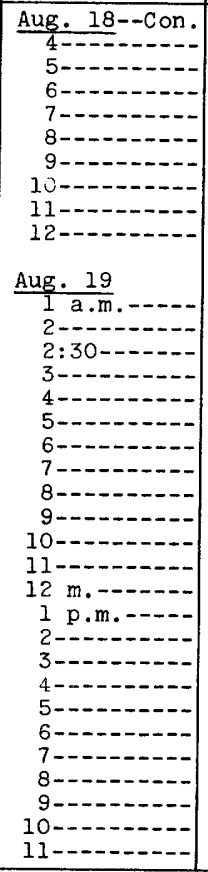 & $\begin{array}{r}4.12 \\
4.52 \\
4.77 \\
5.32 \\
6.30 \\
7.45 \\
8.70 \\
10.00 \\
10.90 \\
\\
11.72 \\
12.59 \\
12.74 \\
12.67 \\
12.39 \\
11.82 \\
11.03 \\
10.40 \\
9.85 \\
9.35 \\
8.90 \\
8.50 \\
8.20 \\
7.90 \\
7.63 \\
7.40 \\
7.18 \\
6.98 \\
6.78 \\
6.60 \\
6.43 \\
9.28 \\
6.13 \\
5.98 \\
\end{array}$ & $\begin{array}{r}1,470 \\
1,870 \\
2,150 \\
2,830 \\
4,280 \\
6,460 \\
9,580 \\
13,700 \\
16,900 \\
\\
19,900 \\
23,200 \\
23,800 \\
23,500 \\
22,400 \\
20,300 \\
17,400 \\
15,100 \\
13,200 \\
11,500 \\
10,200 \\
9,020 \\
8,230 \\
7,490 \\
6,860 \\
6,350 \\
5,890 \\
5,490 \\
5,100 \\
4,790 \\
4,500 \\
4,250 \\
4,010 \\
3,770\end{array}$ & 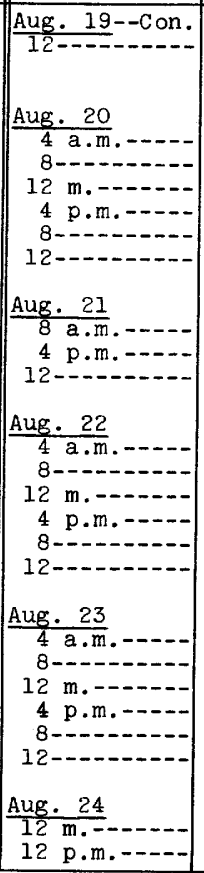 & $\begin{array}{l}3.23 \\
3.16 \\
3.12 \\
3.05 \\
2.98 \\
3.27\end{array}$ & $\begin{array}{l}2,920 \\
2,470 \\
2,120 \\
1,840 \\
1,620 \\
1,420\end{array}$ \\
\hline
\end{tabular}


(248) Delaware River at Montague, N. J.

Location.--Lat $41^{\circ} 18^{\prime} 30^{\prime \prime}$, long $74^{\circ} 47^{\prime} 50^{\prime \prime}$, on right bank at downstream side of old bridge pler and 0.4 mile upstream from toll bridge at Montague, Sussex County, three-quarters of a mile downstream from Saw K1ll. Datum of gage is $369.93 \mathrm{ft}$ above mean sea level, datum of 1929 .

Drainage area. $-3,480 \mathrm{sq} \mathrm{mi}$.

Discharge record.--Stage-discharge relation defined by current-meter measurements below 90,000 cfs. Discharge records being held pending completion of a basin study.

Maxima.--August 1955: Gage height, 35.15 ft 9 a m. Aug. 19.

1940 to July 1955: Discharge, $136,500 \mathrm{cfs}$ May 23, 1942 (gage he1ght, 25.70 $\mathrm{ft}$ ).

Flood of oct. 10, 1903 reached a stage of $35.5 \mathrm{ft}$.

Remarks.--Flow regulated by Lake Wallenpaupack and by Pepacton, Toronto, Cliff Lake, Swinging Bridge, and Neversink Reservolrs (combined capacity, 36,932,000,000 cu ft).

Gage height, in feet, and discharge, in cubic feet per second, at indicated time

\begin{tabular}{|c|c|c|c|c|c|c|c|c|c|c|c|}
\hline TIme & $\begin{array}{c}\text { Gage } \\
\text { helght }\end{array}$ & $\begin{array}{l}\text { *Dis } \\
\text { charge }\end{array}$ & Time & $\begin{array}{c}\text { Gage } \\
\text { height }\end{array}$ & $\begin{array}{l}\text { *Dis- } \\
\text { charge }\end{array}$ & Time & $\begin{array}{c}\text { Gage } \\
\text { helght }\end{array}$ & $\begin{array}{l}\text { *Dis- } \\
\text { charge }\end{array}$ & Time & $\begin{array}{c}\text { Gage } \\
\text { height }\end{array}$ & $\begin{array}{l}\text { *Dis- } \\
\text { charge }\end{array}$ \\
\hline 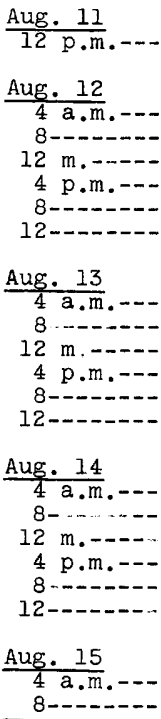 & $\begin{array}{l}4.99 \\
5.44 \\
6.08 \\
7.25 \\
7.87 \\
8.88\end{array}$ & & 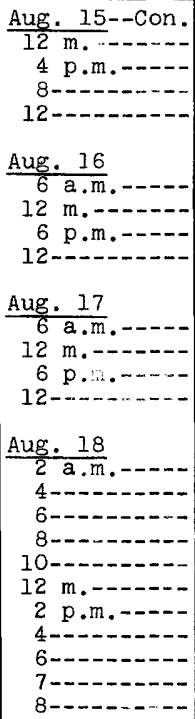 & $\begin{array}{l}8.41 \\
8.36 \\
8.26 \\
7.66 \\
7.21 \\
7.03 \\
6.93 \\
6.68 \\
\\
6.51 \\
6.38 \\
6.28 \\
6.47\end{array}$ & & 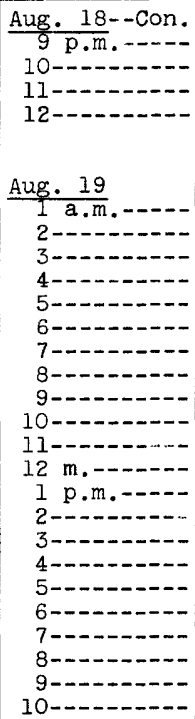 & $\begin{array}{l}19.30 \\
22.80 \\
26.50 \\
28.80 \\
30.70 \\
32.15 \\
33.70 \\
34.50 \\
35.15 \\
35.12 \\
34.92 \\
34.45 \\
33.85 \\
33.50 \\
33.00 \\
32.15 \\
31.00 \\
29.80 \\
28.70 \\
27.73 \\
26.63 \\
25.60\end{array}$ & & $\begin{array}{l}\text { Aug. } 19 \\
11 \mathrm{p} . \mathrm{m} . \\
12-\mathrm{Con} \\
\text { Aug. } 20 \\
1 \mathrm{a} . \mathrm{m} \\
2 \\
3\end{array}$ & $\begin{array}{l}22.75 \\
21.85 \\
21.00 \\
20.28 \\
19.70 \\
19.18 \\
18.72 \\
18.28 \\
17.80 \\
17.40 \\
17.05 \\
16.69 \\
16.39 \\
16.05 \\
15.75 \\
15.50 \\
15.25 \\
15.04 \\
14.82 \\
14.60 \\
14.40 \\
14.20 \\
14.00 \\
13.90\end{array}$ & \\
\hline
\end{tabular}

* Discharge records being held pending completion of a basin study.

(252) Bush Kill at Shoemakers, Pa.

Location.--Lat $41^{\circ} 05^{\prime} 15^{\prime \prime}$, long $75^{\prime \prime} 02: 20^{\prime \prime}$, on right bank at downstream end of wingwall of highway bridge, 0.1 mile downst eam from Saw Creek, three-quarters of a mile northwest of Shoemakers, Monroe County, and 2 miles southwes : of Bushkill. Datum of gage is $421.13 \mathrm{ft}$ above mean sea level, unadjusted.

Dra: nage area --117 sq mi.

Gage-height record.--Water-stage recorder graph except for per1od 3:30 a.m. to 5:30 a.m. Aug. 19. Graph for missing per:od drawn on basis of flocdmaik in gage shelter.

Discharge record. - Stage-discharge relation defined by current-meter measurements below 2,600 cfs and extended to peak stage on basis of slope-area determination. Determinations of the peak discharge by the slope-area method were run on Bush Kill (discharge, $19,500 \mathrm{cfs}$ ) at a site 0.4 mile upstream from the gaging station, and on Saw Creek (discharge, 3,870 cfs) 1.6 miles upstream from 1 ts mouth. Saw Creek enters Bush K1ll immediately above the gaging station. Shifting-control method used at times.

Maxima.--August 1955: Discharge, 23,400 cfs 4 a.m. Aug. 19, (gage he1ght, $13.95 \mathrm{ft}, \mathrm{from}$ floodmark). 1908 to July 1955: Discharge, 5,250 cfs July 24, 1920 (gage helght, 7.2 ft, from graph based on gage readings), from rating curve extended above 2,600 cfs by logarithmic plotting.

Mean discharge, in cubic feet per second, August 1955

\begin{tabular}{|c|c|c|c|c|c|c|c|c|c|c|c|}
\hline Day & Cfs & Day & Cfs & Day & Cfs & Day & $\mathrm{Cfs}$ & Day & Cfs & Day & $\overline{C f s}$ \\
\hline $\begin{array}{l}1 \\
2 \\
3 \\
4 \\
5 \\
6\end{array}$ & $\begin{array}{l}8.2 \\
8.2 \\
7.3 \\
6.8 \\
6.8 \\
6.8\end{array}$ & $\begin{array}{r}7 \\
8 \\
9 \\
10 \\
11\end{array}$ & $\begin{array}{l}9.7 \\
16 \\
15 \\
12 \\
14\end{array}$ & $\begin{array}{l}12 \\
13 \\
14 \\
15 \\
16\end{array}$ & $\begin{array}{r}31 \\
480 \\
584 \\
421 \\
304\end{array}$ & $\begin{array}{l}17 \\
18 \\
19 \\
20 \\
21\end{array}$ & $\begin{array}{r}225 \\
1,110 \\
11,800 \\
4,110 \\
1,980\end{array}$ & $\begin{array}{l}22 \\
23 \\
24 \\
25 \\
26\end{array}$ & $\begin{array}{r}1,270 \\
1,000 \\
744 \\
572 \\
448\end{array}$ & $\begin{array}{l}27 \\
28 \\
29 \\
30 \\
31\end{array}$ & $\begin{array}{l}375 \\
324 \\
272 \\
241 \\
379\end{array}$ \\
\hline
\end{tabular}

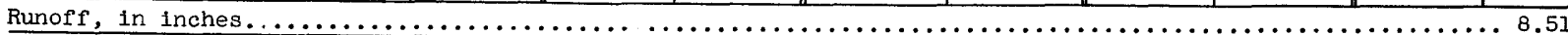


(252) Bush K1ll at Shoemakers, Pa.--Cont1nued

Gage height, in feet, and discharge, in cublc feet per second, at indicated time

\begin{tabular}{|c|c|c|c|c|c|c|c|c|c|c|c|}
\hline Time & $\begin{array}{c}\text { Gage } \\
\text { helght }\end{array}$ & $\begin{array}{l}\text { Dis- } \\
\text { charge }\end{array}$ & Time & $\begin{array}{c}\text { Gage } \\
\text { height }\end{array}$ & $\begin{array}{c}\text { Dis- } \\
\text { charge }\end{array}$ & Time & $\begin{array}{c}\text { Gage } \\
\text { helght }\end{array}$ & $\begin{array}{c}\text { Dis- } \\
\text { charge }\end{array}$ & Time & $\begin{array}{c}\text { Gage } \\
\text { helght }\end{array}$ & $\begin{array}{l}\text { Dis- } \\
\text { charge }\end{array}$ \\
\hline 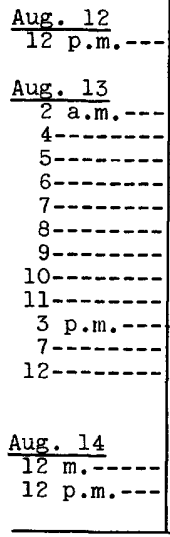 & $\begin{array}{l}1.42 \\
1.56 \\
1.74 \\
1.91 \\
2.15 \\
2.54 \\
2.62 \\
2.77 \\
2.89 \\
2.92 \\
3.03 \\
3.03\end{array}$ & $\begin{array}{r}58 \\
87 \\
134 \\
188 \\
276 \\
443 \\
482 \\
561 \\
628 \\
646 \\
713 \\
713\end{array}$ & 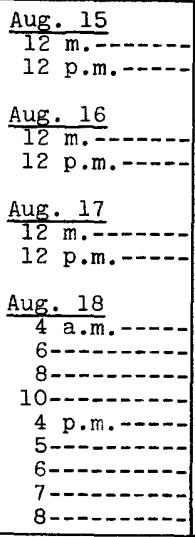 & $\begin{array}{l}1.97 \\
2.07 \\
2.20 \\
2.37 \\
2.63 \\
2.82 \\
3.06 \\
3.64 \\
4.73\end{array}$ & $\begin{array}{r}208 \\
245 \\
295 \\
366 \\
487 \\
588 \\
732 \\
1,150 \\
2,220\end{array}$ & 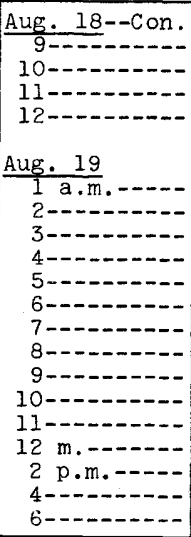 & $\begin{array}{r}5.77 \\
6.42 \\
6.95 \\
7.35 \\
\\
7.95 \\
9.69 \\
12.45 \\
13.95 \\
13.64 \\
12.79 \\
11.79 \\
11.24 \\
10.74 \\
10.34 \\
9.99 \\
9.74 \\
9.30 \\
8.92 \\
8.52\end{array}$ & $\begin{array}{r}3,560 \\
4,550 \\
5,440 \\
6,150 \\
\\
7,300 \\
11,100 \\
18,600 \\
23,400 \\
22,300 \\
19,600 \\
16,700 \\
15,100 \\
13,800 \\
12,800 \\
11,900 \\
11,300 \\
10,200 \\
9,340 \\
8,470\end{array}$ & 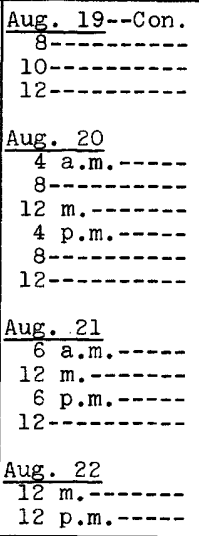 & $\begin{array}{l}6.95 \\
6.53 \\
6.10 \\
5.73 \\
5.40 \\
5.13\end{array}$ & $\begin{array}{l}7,660 \\
6,960 \\
6,340 \\
5,330 \\
4,620 \\
3,930 \\
3,380 \\
2,920 \\
2,580\end{array}$ \\
\hline
\end{tabular}

(254) Flat Brook near Flatbrookville, N. J.

Location.--Lat $41^{\circ} 06^{\prime} 24^{\prime \prime}$, long $74^{\circ} 57^{\prime} 09^{\prime \prime}$, on right bank 1 mile upstream from Flatbrookville, Sussex County, and $1 \frac{1}{2} \mathrm{mlies} u p s t r e a m$ from mouth. Datum of gage is $347.73 \mathrm{ft}$ above mean sea level, datum of 1929 .

Drainage area. $--65.1 \mathrm{sq} \mathrm{mi}$.

Gage-height record.--Water-stage recorder graph except for Aug. 19 (6 a.m. to 6 p.m.) for which graph was drawn on basis of adjoining graphs and high-water mark in gage house and for Aug. $22-25$ when there was no gage-height record.

D1scharge record.--Stage-discharge relation defined by current-meter measurements below 1 , 400 cfs and extended to peak stage on basis of slope-area determination. Discharge for period of no gage-height record Aug. $22-25$ estimated on basis of weather records and records for nearby stations.

Maxima.--August 1955: Discharge, 9,550 cfs 11 a.m. Aug. 19 (gage height, 12.58 ft, from high-water mark in. gage house).

1923 to July 1955: D1scharge, 3,630 cfs Apr. 6, 1952 (gage height, $7.24 \mathrm{ft}$ ). Mean discharge, in cubic feet per second, August 1955

\begin{tabular}{|c|c|c|c|c|c|c|c|c|c|c|c|}
\hline Day & Cfs & Day & $\mathrm{Cfs}$ & Day & $\mathrm{Cfs}$ & Day & $\mathrm{Cf} s$ & Day & Cfs & Day & Cfs \\
\hline $\begin{array}{l}1 \\
2 \\
3 \\
4 \\
5 \\
6\end{array}$ & $\begin{array}{l}8.0 \\
8.0 \\
6.8 \\
6.8 \\
6.8 \\
8.0\end{array}$ & $\begin{array}{r}7 \\
8 \\
9 \\
10 \\
11\end{array}$ & $\begin{array}{l}20 \\
29 \\
18 \\
12 \\
12\end{array}$ & $\begin{array}{l}12 \\
13 \\
14 \\
15 \\
16\end{array}$ & $\begin{array}{r}15 \\
462 \\
506 \\
369 \\
191\end{array}$ & $\begin{array}{l}17 \\
18 \\
19 \\
20 \\
21\end{array}$ & $\begin{array}{r}122 \\
468 \\
6,310 \\
1,510 \\
508\end{array}$ & $\begin{array}{l}22 \\
23 \\
24 \\
25 \\
26\end{array}$ & $\begin{array}{l}260 \\
180 \\
160 \\
140 \\
126\end{array}$ & $\begin{array}{l}27 \\
28 \\
29 \\
30 \\
31\end{array}$ & $\begin{array}{r}112 \\
110 \\
95 \\
86 \\
98\end{array}$ \\
\hline
\end{tabular}

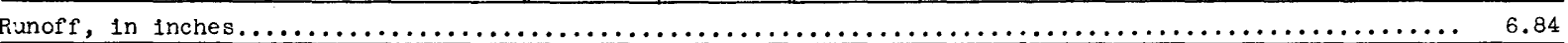

Gage he1ght, in feet, and discharge, in cubic feet per second, at indicated time

\begin{tabular}{|c|c|c|c|c|c|c|c|c|c|c|c|}
\hline Time & $\begin{array}{c}\text { Gage } \\
\text { height }\end{array}$ & $\begin{array}{l}\text { Dis- } \\
\text { charge }\end{array}$ & Time & $\begin{array}{c}\text { Gage } \\
\text { ne1ght }\end{array}$ & $\begin{array}{c}\text { Dis- } \\
\text { charge }\end{array}$ & Time & $\begin{array}{c}\text { Gage } \\
\text { he1ght }\end{array}$ & $\begin{array}{c}\text { Dis- } \\
\text { charge }\end{array}$ & Time & $\begin{array}{c}\text { Gage } \\
\text { height }\end{array}$ & $\begin{array}{l}\text { Dis- } \\
\text { charge }\end{array}$ \\
\hline 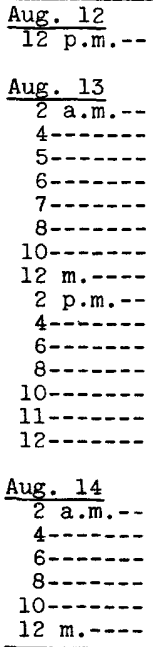 & $\begin{array}{l}2.04 \\
2.14 \\
2.24 \\
2.39 \\
2.66 \\
2.92 \\
3.26 \\
3.43 \\
3.60 \\
3.75 \\
3.96 \\
4.19 \\
4.35 \\
4.37 \\
4.34\end{array}$ & $\begin{array}{r}30 \\
43 \\
58 \\
86 \\
159 \\
249 \\
392 \\
470 \\
555 \\
632 \\
743 \\
874 \\
970 \\
982 \\
964\end{array}$ & 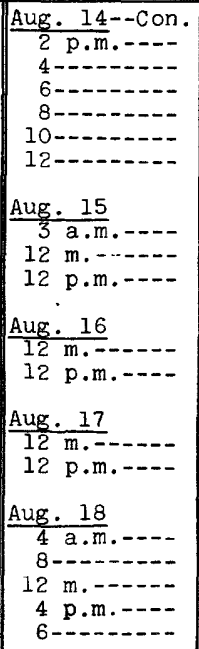 & $\begin{array}{l}3.08 \\
3.20 \\
3.37 \\
3.41 \\
3.40 \\
3.43\end{array}$ & $\begin{array}{l}312 \\
365 \\
442 \\
460 \\
455 \\
470\end{array}$ & $\begin{array}{l}\text { Aug. } 18-1 \text { Con. } \\
7 \\
8 \\
9 \\
10 \\
11 \\
12\end{array}$ & $\begin{array}{l}6.75 \\
7.45 \\
8.25 \\
8.95 \\
9.50 \\
10.2 \\
10.8 \\
11.3 \\
11.8 \\
12.4 \\
12.58 \\
12.5 \\
12.3 \\
11.9 \\
11.4 \\
10.9 \\
10.5 \\
10.13\end{array}$ & $\begin{array}{l}2,720 \\
3,370 \\
4,180 \\
4,940 \\
5,600 \\
6,440 \\
7,190 \\
7,840 \\
8,490 \\
9,310 \\
9,550 \\
9,450 \\
9,170 \\
8,620 \\
7,970 \\
7,320 \\
6,800 \\
6,360\end{array}$ & 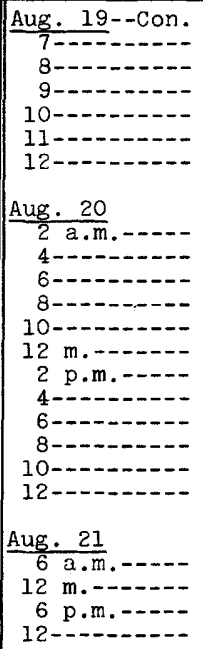 & $\begin{array}{l}6.75 \\
6.25 \\
5.76 \\
5.44 \\
5.13 \\
4.86 \\
4.65 \\
4.46 \\
4.30 \\
4.17 \\
4.05 \\
3.92 \\
\\
\\
3.64 \\
3.44 \\
3.29 \\
3.35\end{array}$ & $\begin{array}{r}2,720 \\
2,300 \\
1,920 \\
1,690 \\
1,470 \\
1,290 \\
1,160 \\
1,040 \\
940 \\
862 \\
792 \\
721\end{array}$ \\
\hline
\end{tabular}


(260) Brodhead Creek at Minisink H1lls, Pa.

Location.--Lat $41^{\circ} 00^{\prime} 00^{\prime \prime}$, long $75^{\circ} 08^{\prime} 50^{\prime \prime}$, on right bank $175 \mathrm{ft}$ downstream from maln building of Coates Paper Box Co. at Minisink Hills, Monroe County, 1 mile upstream from mouth and 3 miles southeast of East Stroudsburg. Datum of gage is 304.03 ft above mean sea level, datum of 1929 .

Drainage area. $--259 \mathrm{sq} \mathrm{mi}$.

Gage-helight record. - Water-stage recorder graph except for period Aug. 5-31. Graph based on readings from reference points and floodmarks Aug. 18, 19 and, during period Aug. 20-31, on occasional staff gage readings and shape of general recession.

Discharge record.--Stage-discharge relation defined by current-meter measurements below 5,000 cf s and extended above on basis of computation of flow over dam at gage height $14.43 \mathrm{ft}$ and slope-area determination at gage helght $29.9 \mathrm{ft}$. Discharge for days of no gage-height record Aug. 5-17 was estimated on basis of records for nearby stations.

Maxima. - August 1955: Discharge 68,800 cfs 2 a.m. Aug. 19 (gage he1ght, 29.9 ft).

1950 to July 1955: Discharge, 19,900 cfs Dec. 11, 1952 (gage height, 14.43 ft), from computation of peak flow over dam.

\begin{tabular}{|c|c|c|c|c|c|c|c|c|c|c|c|}
\hline $\mathrm{Da}$. & Cfs & $\mathrm{Da}_{j}$ & Cfs & Day & Cfs & Day & Cfs & $\overline{\mathrm{DaJ}}$ & Cfs & Day & Cfs \\
\hline $\begin{array}{l}1 \\
2 \\
3 \\
4 \\
5 \\
6\end{array}$ & $\begin{array}{l}51 \\
50 \\
46 \\
45 \\
41 \\
41\end{array}$ & $\begin{array}{r}7 \\
8 \\
9 \\
10 \\
11\end{array}$ & $\begin{array}{l}60 \\
96 \\
90 \\
77 \\
87\end{array}$ & $\begin{array}{l}12 \\
13 \\
14 \\
15 \\
16\end{array}$ & $\begin{array}{r}190 \\
3,000 \\
3,500 \\
2,600 \\
2,000\end{array}$ & $\begin{array}{l}17 \\
18 \\
19 \\
20 \\
21\end{array}$ & $\begin{array}{r}1,300 \\
6,730 \\
30,500 \\
9,970 \\
5,020\end{array}$ & $\begin{array}{l}22 \\
23 \\
24 \\
25 \\
26\end{array}$ & $\begin{array}{r}2,710 \\
2.190 \\
1,990 \\
1,190 \\
815\end{array}$ & $\begin{array}{l}27 \\
28 \\
29 \\
30 \\
31\end{array}$ & $\begin{array}{l}662 \\
594 \\
542 \\
575 \\
882\end{array}$ \\
\hline
\end{tabular}

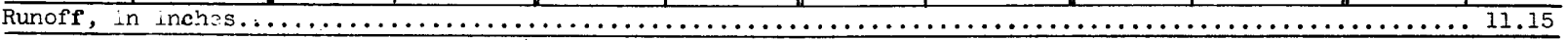

\begin{tabular}{|c|c|c|c|c|c|c|c|c|c|c|c|}
\hline Time & $\begin{array}{c}\text { Gage } \\
\text { height }\end{array}$ & $\begin{array}{c}\text { Dis- } \\
\text { charge }\end{array}$ & Time & $\begin{array}{c}\text { Gage } \\
\text { he1ght }\end{array}$ & $\begin{array}{l}\text { Dis- } \\
\text { charge }\end{array}$ & Time & $\begin{array}{c}\text { Gage } \\
\text { he1ght }\end{array}$ & $\begin{array}{l}\text { Dis- } \\
\text { charge }\end{array}$ & Time & $\begin{array}{c}\text { Gage } \\
\text { height }\end{array}$ & $\begin{array}{l}\text { Dis- } \\
\text { charge }\end{array}$ \\
\hline 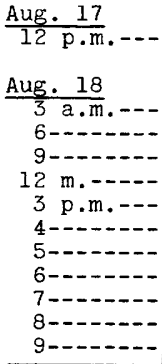 & $\begin{array}{r}2.50 \\
2.65 \\
2.88 \\
2.97 \\
3.12 \\
3.25 \\
3.48 \\
3.84 \\
4.45 \\
8.00 \\
15.00\end{array}$ & $\begin{array}{r}1,120 \\
1,220 \\
1,390 \\
1,470 \\
1,590 \\
1,690 \\
1,880 \\
2,210 \\
2,760 \\
7,130 \\
21,400\end{array}$ & $\begin{array}{l}\text { Aug. } 18-- \text { Con. } \\
10 \\
11 \\
12 \\
\text { Aug. } 19 \\
1 \text { a.m. } \\
2 \\
3 \\
4 \\
6 \\
7 \\
9\end{array}$ & $\begin{array}{l}20.70 \\
22.30 \\
23.85 \\
\\
26.00 \\
29.9 \\
26.80 \\
24.30 \\
22.55 \\
21.10 \\
20.10 \\
19.15 \\
18.40\end{array}$ & $\begin{array}{l}37,800 \\
42,600 \\
47,200 \\
\\
54,600 \\
68,800 \\
57,400 \\
48,600 \\
43,400 \\
39,000 \\
36,000 \\
33,200 \\
30,900\end{array}$ & $\begin{array}{r}\text { Aug. } 19--C o n . \\
10 \\
11 \\
12 \text { m. } \\
1 \text { p.m. } \\
210 \\
3 \\
4 \\
5 \\
6 \\
8 \\
10 \\
12\end{array}$ & $\begin{array}{l}17.65 \\
17.05 \\
16.45 \\
15.90 \\
15.42 \\
15.00 \\
14.59 \\
14.22 \\
13.85 \\
13.18 \\
12.55 \\
12.00\end{array}$ & $\begin{array}{l}28,700 \\
27,000 \\
25,300 \\
23,800 \\
22,500 \\
21,400 \\
20,400 \\
19,500 \\
18,600 \\
17,000 \\
15,500 \\
14,300\end{array}$ & 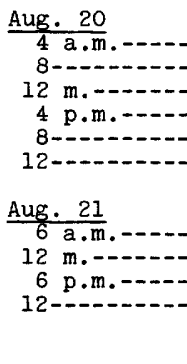 & $\begin{array}{r}11.10 \\
10.35 \\
9.75 \\
9.29 \\
8.80 \\
8.32\end{array}$ & $\begin{array}{r}12,300 \\
10,800 \\
9,580 \\
8,710 \\
7,820 \\
6,970\end{array}$ \\
\hline
\end{tabular}


(267) Paulins Kill at Bla1rstown, N. J.

Location.--Lat $40^{\circ} 58^{\prime} 44^{\prime \prime}$, long $74^{\circ} 57^{\prime} 15^{\prime \prime}$, on right bank 1,200 ft upstream from bridge on State Highway 94 in Blairstown, Warren County, 1,400 ft upstream from Blairs Creek, and 10 miles upstream from mouth. Datum of gage $1 \mathrm{~s}$ $335.86 \mathrm{ft}$ above mean sea level, datum of 1929.

Drainage area. $--126 \mathrm{sq} \mathrm{mi}$.

Gage-helght record. --Water-stage recorder graph except Aug. 19 (8 a.m. to 3 p.m.) for which graph was drawn on basis of adjoining graphs and high-water mark in gage house.

D1scharge record.--Stage-d1scharge relation defined below 6,100 cfs and extended to peak stage on bas1s of slopearea determination.

Maxima.--August 1955: Discharge, $8,740 \mathrm{cfs} 12 \mathrm{~m}$. Aug. 19 (gage he1ght, $11.12 \mathrm{ft}$, from high-water mark in gage house).

1921 to July 1955: Discharge, $4,480 \mathrm{cfs}$ Sept. 22,1938 (gage he1ght, $7.56 \mathrm{ft}$ ).

Remarks.--Diurnal fluctuation caused by powerplant above station and flow slightly regulated by Swartswood Lake. Mean discharge, in cuble feet per second, August 1955

\begin{tabular}{|c|c|c|c|c|c|c|c|c|c|c|c|}
\hline Day & $\mathrm{Cfs}$ & Day & Cfs & Day & $\mathrm{Cfs}$ & Day & $\mathrm{Cfs}$ & Day & Cfs & Day & $\mathrm{Cfs}$ \\
\hline $\begin{array}{l}1 \\
2 \\
3 \\
4 \\
5 \\
6\end{array}$ & $\begin{array}{c}17 \\
9.1 \\
14 \\
12 \\
12 \\
9.1 \\
\end{array}$ & $\begin{array}{r}7 \\
8 \\
9 \\
10 \\
11\end{array}$ & $\begin{array}{l}17 \\
38 \\
28 \\
16 \\
20\end{array}$ & $\begin{array}{l}12 \\
13 \\
14 \\
15 \\
16\end{array}$ & $\begin{array}{r}22 \\
299 \\
671 \\
558 \\
336\end{array}$ & $\begin{array}{l}17 \\
18 \\
19 \\
20 \\
21\end{array}$ & $\begin{array}{r}226 \\
409 \\
5,950 \\
3,750 \\
2,380\end{array}$ & $\begin{array}{l}22 \\
23 \\
24 \\
25 \\
26\end{array}$ & $\begin{array}{r}1,700 \\
1,110 \\
712 \\
506 \\
408\end{array}$ & $\begin{array}{l}27 \\
28 \\
29 \\
30 \\
31\end{array}$ & $\begin{array}{l}345 \\
292 \\
249 \\
221 \\
216\end{array}$ \\
\hline
\end{tabular}

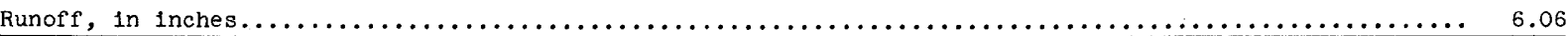

Gage height, in feet, and discharge, in cubic feet per second, at indicated time

\begin{tabular}{|c|c|c|c|c|c|c|c|c|c|c|c|}
\hline Time & $\begin{array}{c}\text { Gage } \\
\text { height }\end{array}$ & $\begin{array}{c}\text { Dis- } \\
\text { charge }\end{array}$ & Time & $\begin{array}{c}\text { Gage } \\
\text { height }\end{array}$ & $\begin{array}{c}\text { Dis- } \\
\text { charge }\end{array}$ & TIme & $\begin{array}{l}\text { Gage } \\
\text { eight }\end{array}$ & $\begin{array}{c}\text { D1s- } \\
\text { charge }\end{array}$ & Time & & $\begin{array}{c}\text { Dis- } \\
\text { charge }\end{array}$ \\
\hline 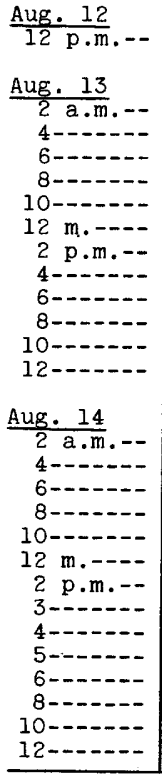 & $\begin{array}{l}3.29 \\
3.18 \\
3.08 \\
3.02 \\
2.95 \\
2.86 \\
2.99 \\
3.24 \\
3.42 \\
3.45 \\
3.44 \\
3.39 \\
3.29 \\
3.18\end{array}$ & $\begin{array}{l}714 \\
670 \\
630 \\
606 \\
578 \\
541 \\
594 \\
694 \\
766 \\
778 \\
774 \\
754 \\
714 \\
670\end{array}$ & 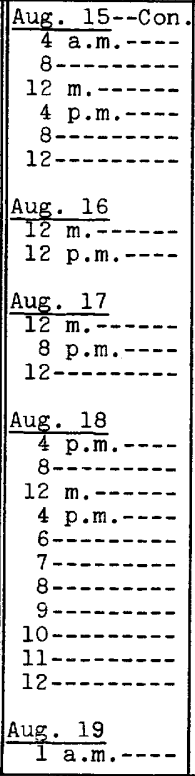 & $\begin{array}{l}2.14 \\
2.21 \\
2.28 \\
2.40 \\
2.46 \\
2.62 \\
2.94 \\
3.40 \\
4.08 \\
4.80 \\
5.42\end{array}$ & $\begin{array}{r}199 \\
230 \\
265 \\
331 \\
361 \\
437 \\
573 \\
758 \\
1,060 \\
1,410 \\
1,760\end{array}$ & 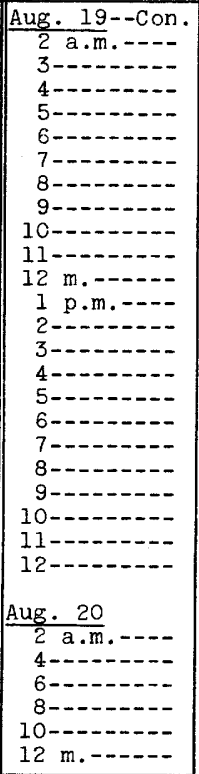 & $\begin{array}{c}6.40 \\
7.02 \\
7.70 \\
8.40 \\
9.10 \\
9.56 \\
10.0 \\
10.4 \\
10.8 \\
11.1 \\
11.12 \\
11.1 \\
10.9 \\
10.6 \\
10.32 \\
10.02 \\
9.76 \\
9.57 \\
9.38 \\
9.20 \\
9.02 \\
8.88 \\
8.74\end{array}$ & $\begin{array}{l}2,240 \\
2,970 \\
3,670 \\
4,480 \\
5,390 \\
6,040 \\
6,700 \\
7,380 \\
8,160 \\
8,700 \\
8,740 \\
8,700 \\
8,310 \\
7,740 \\
7,240 \\
6,730 \\
6,340 \\
6,060 \\
5,780 \\
5,530 \\
5,280 \\
5,090 \\
4,910\end{array}$ & 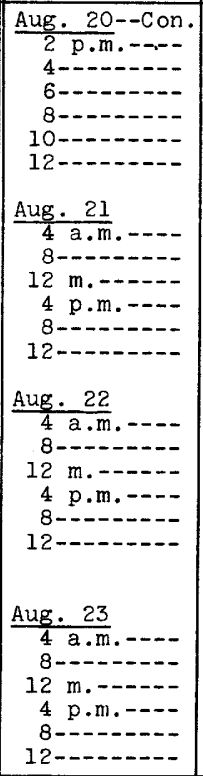 & $\begin{array}{l}6.72 \\
6.51 \\
6.28 \\
6.06 \\
5.85 \\
5.77\end{array}$ & $\begin{array}{l}2,700 \\
2,530 \\
2,350 \\
2,190 \\
2,040 \\
1,990 \\
\\
1,910 \\
1,820 \\
1,740 \\
1,590 \\
1,480 \\
1,380\end{array}$ \\
\hline
\end{tabular}


Location.--Lat $40^{\circ} 49^{\prime} 36^{\prime \prime}$, Iong $75^{\circ} \mathrm{C} 5^{\prime} 02^{\prime \prime}$, on left bank at Belvidere, Warren County, 1,500 ft downstream from Pequest River. Datum of gage is $226.43 \mathrm{ft}$ above mean sea level, datum of 1929 .

Drainage area. $--4,535 \mathrm{sq} \mathrm{mi}$.

Gage-he1ght record.--Water-stage recorder graph except Aug. 19 (5 p.m.) to Aug. 20 (12 m.) when graph was drawn on basis of hourly wire-weight gage readings by Delaware River Joint Toll Bridge Commission and high-water mark in gage house.

Discharge record.--Stage-discharge relation defined by current-meter measurements below 170,000 cfs and extended to peak stage on basis of slope-area determination.

Maxima.--August 1955: Discharge, 247,000 of s 8 p.m. Aug. 19 (gage he1ght, $30.21 \mathrm{ft}$, from high-water mark in gage house).

1922 to July 1955: Discharge, 179,000 cf's Mar. 19, 1936 (gage height, 25.0 ft).

Flood of oct. 10, 1903 , reached a stage of $28.6 \mathrm{ft}$, from floodmark (discharge, $220,000 \mathrm{cfs}$ )

Remarks.--Flow regulated by Lake Wallenpaupack and by Pepacton, Toronto, Cliff Lake, Swinging Bridge, and Neversink

Reservolrs (combined capacity, 36,932,000,000 cu ft).

Mean discharge, in cubic feet per second, August 1955

\begin{tabular}{|c|c|c|c|c|c|c|c|c|c|c|c|}
\hline Day & $\mathrm{Cfs}$ & Day & Cfs & Day & $\mathrm{Cfs}$ & Day & Cfs & Day & Cfs & Day & $\mathrm{Cfs}$ \\
\hline $\begin{array}{l}1 \\
2 \\
3 \\
4 \\
5 \\
6\end{array}$ & $\begin{array}{r}1,160 \\
940 \\
1,480 \\
1,670 \\
1,520 \\
1,560\end{array}$ & $\begin{array}{r}7 \\
8 \\
9 \\
10 \\
11\end{array}$ & $\begin{array}{l}1,520 \\
1,220 \\
1,120 \\
1,120 \\
1,060\end{array}$ & $\begin{array}{l}12 \\
13 \\
14 \\
15 \\
16\end{array}$ & $\begin{array}{r}1,410 \\
5,210 \\
16,200 \\
15,900 \\
11,900\end{array}$ & $\begin{array}{l}17 \\
18 \\
19 \\
20 \\
21\end{array}$ & $\begin{array}{r}7,980 \\
9,230 \\
178,000 \\
134,000 \\
45,500\end{array}$ & $\begin{array}{l}22 \\
23 \\
24 \\
25 \\
26\end{array}$ & $\begin{array}{l}29,300 \\
25,300 \\
21,600 \\
16,200 \\
12,900\end{array}$ & $\begin{array}{l}27 \\
28 \\
29 \\
30 \\
31\end{array}$ & $\begin{array}{r}10,800 \\
9,240 \\
7,900 \\
7,240 \\
7,720\end{array}$ \\
\hline
\end{tabular}

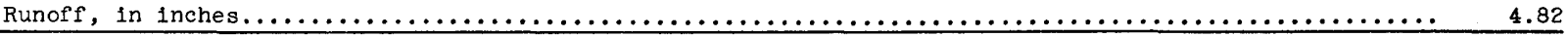

Gage height, in feet, and discharge, in cubic feet per second, at indicated time

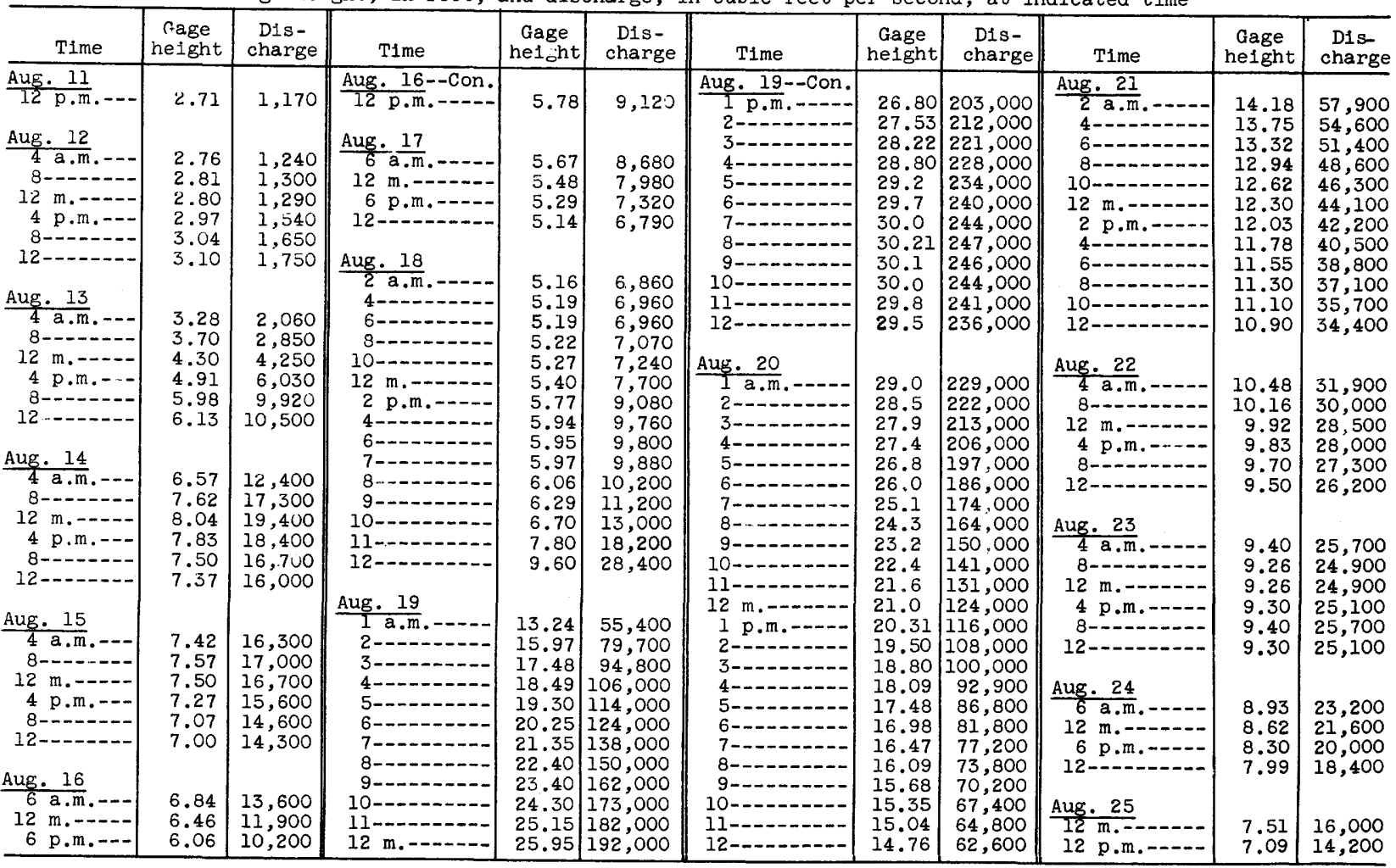

(272) Lehigh River at Stoddartsville, Pa.

Location.--Lat $41^{\circ} 07^{\prime} 45^{\prime \prime}$, Iong $75^{\circ} 37^{\prime} 40^{\prime \prime}$, nn left bank 75 ft upstream from concrete bridge on state Highway 115 , at Stoddartsville, Luzerne County, 1.9 miles upstream from Tobyhanna Creek, and 4 miles southwest of Thornhurst. Datum of gage is $1,463.81 \mathrm{ft}$ above mean sea level, datum of 1929 , supplementary adjustment of 1943 .

Drainage area. $--91.7 \mathrm{sq} \mathrm{mi}$

Gage-helght record. - Water-stage recorder graph except for period Aug. 19, 20 when graph was drawn based on elevation of crest stage and partial gage-height record.

Discharge record.--Stage-discharge relation defined by current-meter measurements below 1,700 cfs and extended to peak itage on basis of slope-area determination. Shifting-control method used at times.

Maxima.--August 1955: Discharge, 31,900 cfs 4 a.m. Aug. 19 (gage helght $16.37 \mathrm{ft}$, from floodmarks).

1943 to July 1955: Discharge, 7,250 cfs (revised) Dec. 4, 1950 (gage height, 8.59 ft) from rating curve extended as explained above.

Flood of May 22, 1942, reached a stage of $12.03 \mathrm{ft}$, present s1te and datum (discharge, 15,700 cfs, revised,

from rating curve extended as explained above).

Mean discharge, in cubic feet per second, August 1955

\begin{tabular}{|c|c|c|c|c|c|c|c|c|c|c|c|}
\hline Day & $\mathrm{Cfs}$ & Day & Cfs & Day & Cfs & Day & Cfs & Day & $\mathrm{Cfs}$ & Day & Cfs \\
\hline $\begin{array}{l}1 \\
2 \\
3 \\
4 \\
5 \\
6\end{array}$ & $\begin{array}{l}14 \\
14 \\
13 \\
13 \\
13 \\
14\end{array}$ & $\begin{array}{r}7 \\
8 \\
9 \\
10 \\
11\end{array}$ & $\begin{array}{l}14 \\
25 \\
19 \\
15 \\
23\end{array}$ & $\begin{array}{l}12 \\
13 \\
14 \\
15 \\
16\end{array}$ & $\begin{array}{r}74 \\
978 \\
2,190 \\
1,520 \\
660\end{array}$ & $\begin{array}{l}17 \\
18 \\
19 \\
20 \\
21\end{array}$ & $\begin{array}{r}372 \\
2,030 \\
18,900 \\
2,850 \\
1,110\end{array}$ & $\begin{array}{l}22 \\
23 \\
24 \\
25 \\
26\end{array}$ & $\begin{array}{l}702 \\
562 \\
401 \\
303 \\
240\end{array}$ & $\begin{array}{l}27 \\
28 \\
29 \\
30 \\
31\end{array}$ & $\begin{array}{l}209 \\
180 \\
167 \\
155 \\
360\end{array}$ \\
\hline
\end{tabular}

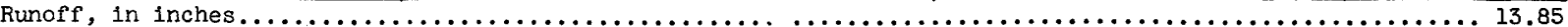


(272) Leh1gh River at Stoddartsville, Pa.--Continued

Gage helght, in feet, and discharge, in cublc feet per second, at indicated time

\begin{tabular}{|c|c|c|c|c|c|c|c|c|c|c|c|}
\hline Time & $\begin{array}{c}\text { Gage } \\
\text { he1ght }\end{array}$ & $\begin{array}{l}\text { Dis- } \\
\text { charge }\end{array}$ & Time & $\begin{array}{c}\text { Gage } \\
\text { he1ght }\end{array}$ & $\begin{array}{l}\text { D1s- } \\
\text { charge }\end{array}$ & Time & $\begin{array}{c}\text { Gage } \\
\text { he1ght }\end{array}$ & $\begin{array}{c}\text { Dis- } \\
\text { charge }\end{array}$ & Time & $\begin{array}{c}\text { Gage } \\
\text { helght }\end{array}$ & $\begin{array}{l}\text { D1s- } \\
\text { charge }\end{array}$ \\
\hline 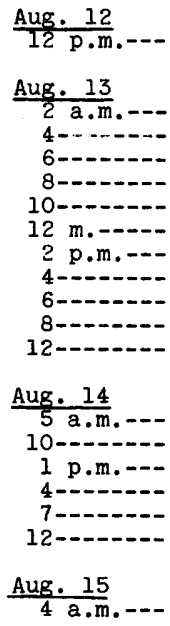 & $\begin{array}{l}1.08 \\
1.18 \\
1.46 \\
1.77 \\
2.27 \\
2.92 \\
3.63 \\
4.34 \\
4.73 \\
4.90 \\
4.94\end{array}$ & $\begin{array}{r}76 \\
94 \\
157 \\
247 \\
439 \\
749 \\
1,160 \\
1,640 \\
1,950 \\
2,100 \\
2,140 \\
\\
2,250 \\
2,020 \\
1,910 \\
2,190 \\
2,450 \\
2,270\end{array}$ & 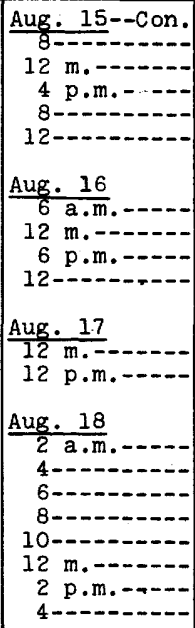 & $\begin{array}{l}4.42 \\
4.08 \\
3.78 \\
3.49 \\
3.24 \\
\\
2.96 \\
2.70 \\
2.50 \\
2.31 \\
\\
2.09 \\
1.93 \\
\\
1.93 \\
2.08 \\
2.24 \\
2.40 \\
2.64 \\
2.80 \\
2.92 \\
3.17\end{array}$ & $\begin{array}{r}1,710 \\
1,460 \\
1,250 \\
1,070 \\
924 \\
\\
770 \\
638 \\
543 \\
456 \\
\\
\\
364 \\
303\end{array}$ & $\begin{array}{l}\text { Aug. } 18 \text { Con. } \\
5 \\
6 \\
7 \\
8 \\
9 \\
10 \\
11 \\
12-\ldots\end{array}$ & $\begin{array}{l}14.50 \\
15.55 \\
16.10 \\
16.37 \\
16.30 \\
15.95 \\
15.45 \\
15.10 \\
14.75 \\
14.50 \\
14.00 \\
13.55 \\
13.00 \\
12.45 \\
11.90\end{array}$ & $\begin{array}{r}1,100 \\
1,600 \\
2,190 \\
3,390 \\
4,800 \\
7,470 \\
11,000 \\
17,000 \\
\\
24,200 \\
28,400 \\
30,700 \\
31,900 \\
31,600 \\
30,000 \\
28,000 \\
26,600 \\
25,200 \\
24,200 \\
22,300 \\
20,700 \\
18,800 \\
17,000 \\
15,300\end{array}$ & 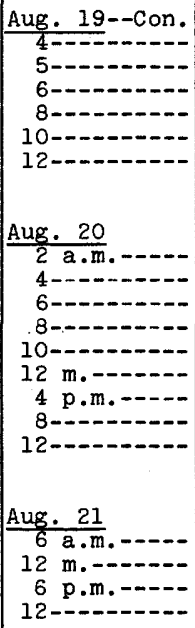 & $\begin{array}{l}7.67 \\
7.34 \\
7.02 \\
6.72 \\
6.43 \\
6.24 \\
5.63 \\
5.19 \\
4.88\end{array}$ & $\begin{array}{l}4,720 \\
4,180 \\
3,680 \\
3,250 \\
2,870 \\
2,630 \\
2,040 \\
1,680 \\
1,460\end{array}$ \\
\hline
\end{tabular}

(275) Lehigh River at Tannery, Pa.

Location.--Lat $41^{\circ} \mathrm{O} 2 \cdot 25^{\prime \prime}$, long $75^{\circ} 45^{\prime} 45^{\prime \prime}$, on right bank $600 \mathrm{ft}$ upstream from highway bridge at Tannery, Luzerne

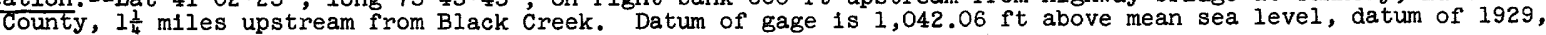
supplementary adjustment of 1943.

Drainage area. $-322 \mathrm{sq} \mathrm{mi}$.

Gage-helght record. --Water-stage recorder graph except for period Aug. 17-24 when graph was reconstructed on basis of floodmarks and hydrographic comparison with upstream and downstream stations.

Discharge record. --Stage-discharge relation defined by current-met $\mathrm{r}$ measurements below $7,100 \mathrm{cfs}$ and extended above on basis of siope-area determination at peak stage made at white Haven, 2 miles upstream (discharge, 57,300 cfs), Aug. 22-24 discharge estimated on basis of records for upstream and downstream stations. Shifting-control method used at times.

Maxima.--August i955: D1scharge, $58,300 \mathrm{cfs} 5 \mathrm{a} . \mathrm{m}$. Aug. 19 (gage height $22.2 \mathrm{ft}$, from floodmark).

i914 to July 1955: Discharge, 29,600 cfs May 22, 1942 (gage helght, $16.51 \mathrm{ft}$ ), from computation of peak flow over dam.

Mean discharge, in cublc feet per second, August 1955

\begin{tabular}{|c|c|c|c|c|c|c|c|c|c|c|c|}
\hline Day & Cfs & Day & Cf's & Day & Cfs & Day & Cfs & Day & Cfs & Day & Cfs \\
\hline $\begin{array}{l}1 \\
2 \\
3 \\
4 \\
5 \\
6\end{array}$ & $\begin{array}{l}66 \\
66 \\
64 \\
64 \\
68 \\
74 \\
\end{array}$ & $\begin{array}{r}7 \\
8 \\
9 \\
10 \\
11\end{array}$ & $\begin{array}{l}74 \\
86 \\
84 \\
74 \\
96\end{array}$ & $\begin{array}{l}12 \\
13 \\
14 \\
15 \\
16\end{array}$ & $\begin{array}{l}134 \\
1,370 \\
6,140 \\
4,180 \\
2,140\end{array}$ & $\begin{array}{l}17 \\
18 \\
19 \\
20 \\
21\end{array}$ & $\begin{array}{r}1,220 \\
2,740 \\
36,000 \\
8,060 \\
3,240\end{array}$ & $\begin{array}{l}22 \\
23 \\
24 \\
25 \\
26\end{array}$ & $\begin{array}{r}2,200 \\
1,700 \\
1,250 \\
914 \\
730\end{array}$ & $\begin{array}{l}27 \\
28 \\
29 \\
30 \\
31\end{array}$ & $\begin{array}{r}618 \\
528 \\
468 \\
469 \\
1,200\end{array}$ \\
\hline
\end{tabular}

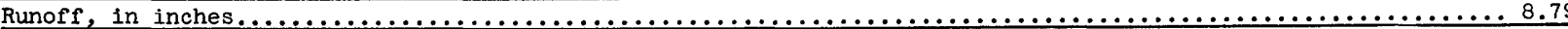

Gage helght, in feet, and discharge, in cubic feet per second, at indicated time

\begin{tabular}{|c|c|c|c|c|c|c|c|c|c|c|c|}
\hline & ght & $\begin{array}{c}\text { Dis- } \\
\text { harge }\end{array}$ & Time & $\begin{array}{l}\text { Gage } \\
\text { height }\end{array}$ & $\begin{array}{c}\text { Dis- } \\
\text { charge }\end{array}$ & Time & $\begin{array}{c}\text { Gage } \\
\text { height }\end{array}$ & $=\begin{array}{c}\text { Dis- } \\
\text { charge }\end{array}$ & Time & & $\begin{array}{l}\text { Dis- } \\
\text { charge }\end{array}$ \\
\hline 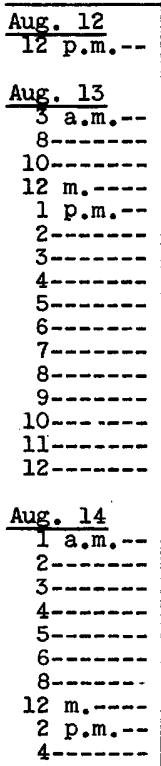 & $\begin{array}{l}1.35 \\
1.44 \\
1.56 \\
1.78 \\
2.15 \\
2.59 \\
3.02 \\
3.45 \\
3.90 \\
4.22 \\
4.54 \\
4.78 \\
5.25 \\
5.90 \\
6.55 \\
6.98\end{array}$ & $\begin{array}{r}138 \\
175 \\
234 \\
364 \\
632 \\
982 \\
1,360 \\
1,750 \\
2,180 \\
2,480 \\
2,790 \\
3,030 \\
3,500 \\
4,190 \\
4,920 \\
5,440\end{array}$ & 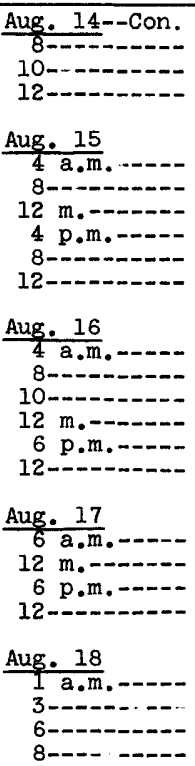 & $\begin{array}{l}6.80 \\
6.28 \\
5.78 \\
5.35 \\
4.97 \\
4.65\end{array}$ & $\begin{array}{l}2,610 \\
2,360 \\
2,220 \\
2,090 \\
1,790 \\
1,520\end{array}$ & 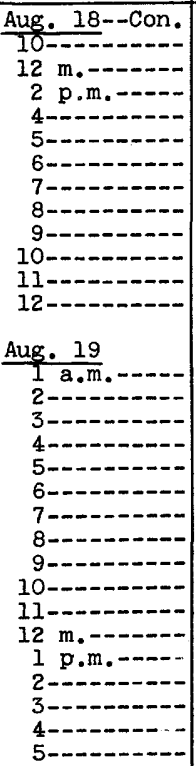 & $\begin{array}{r}3.37 \\
3.52 \\
3.72 \\
4.03 \\
4.30 \\
4.59 \\
5.05 \\
5.95 \\
6.95 \\
8.10 \\
9.10 \\
10.90 \\
\\
13.40 \\
17.35 \\
19.55 \\
21.50 \\
22.20 \\
22.00 \\
21.65 \\
21.10 \\
20.65 \\
20.15 \\
19.70 \\
19.20 \\
18.65 \\
18.10 \\
17.50 \\
16.75 \\
.6 .05\end{array}$ & $\begin{array}{l}1,670 \\
1,810 \\
2,000 \\
2,300 \\
2,560 \\
2,840 \\
3,300 \\
4,240 \\
5,400 \\
6,950 \\
8,610 \\
12,300 \\
\\
19,200 \\
33,400 \\
43,800 \\
54,100 \\
58,300 \\
57,100 \\
55,000 \\
51,700 \\
49,200 \\
46,800 \\
44,500 \\
42,000 \\
39,200 \\
36,500 \\
34,000 \\
31,000 \\
28,200\end{array}$ & 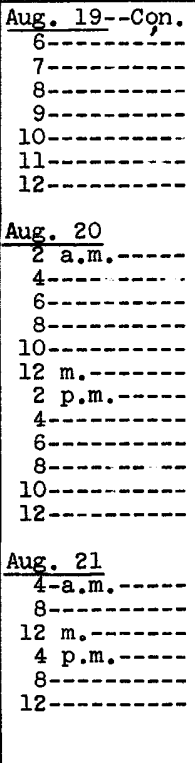 & $\begin{array}{r}15.45 \\
14.80 \\
14.25 \\
13.70 \\
13.1 .5 \\
12.67 \\
12.16 \\
\\
11.26 \\
10.58 \\
9.77 \\
9.15 \\
8.69 \\
8.22 \\
7.80 \\
7.43 \\
7.12 \\
6.83 \\
6.55 \\
6.27\end{array}$ & $\begin{array}{r}13,200 \\
11,500 \\
9,890 \\
8,700 \\
7,890 \\
7,130 \\
6,510 \\
6,010 \\
5,610 \\
5,260 \\
4,920 \\
4,600\end{array}$ \\
\hline
\end{tabular}


(280) Lehigh River at Walnutport, $\mathrm{Pa}$.

Location.--Lat $40^{\circ} 45^{\prime \prime} 20^{\prime \prime}$, long $75^{\circ} 36^{\prime} 15^{\prime \prime}$, on left bank 0.3 mile upstream from highway bridge at Walnutport, Northampton County, and $0.4 \mathrm{mile}$ upstream from Trout Creek. Datum of gage is $350.27 \mathrm{ft}$ above mean sea level, datum of 1929 , supplementary adjustment of 1943.

Drainage area. --889 sq $\mathrm{ml}$.

Gage-height record.--Water-stage recorder graph except for 15 hours on Aug. 19 when graph was drawn on basis of floodmark of peak and fragmentary gage-helght record.

Discharge record.--Stage-discharge relation defined by current-meter measurements below 60,000 cfs and extended to peak stage on basis of logarithmic plotting.

Maxima.--August 1955: Discharge, 77,800 cfs 6:30 a m. Aug. 19 (gage helght, $17.68 \mathrm{ft}$, from floodmark). 1946 to July 1955: Maximum discharge, 46,000 cfs Dec. 4, 1950 (gage height, $12.66 \mathrm{ft}$ ). Maximum stage known, 20.6 ft May 23, 1942, from floodmarks.

Mean discharge, in cubic feet per second, August 1955

\begin{tabular}{|c|c|c|c|c|c|c|c|c|c|c|c|}
\hline Day & Cfs & Day & Cfs & Day & Cfs & Day & $\mathrm{Cfs}$ & Day & Cfs & Day & Cfs \\
\hline $\begin{array}{l}1 \\
2 \\
3 \\
4 \\
5 \\
6\end{array}$ & $\begin{array}{l}228 \\
224 \\
212 \\
212 \\
216 \\
220\end{array}$ & $\begin{array}{r}7 \\
8 \\
9 \\
10 \\
11\end{array}$ & $\begin{array}{l}236 \\
265 \\
250 \\
228 \\
343\end{array}$ & $\begin{array}{l}12 \\
13 \\
14 \\
15 \\
16\end{array}$ & $\begin{array}{r}462 \\
3,560 \\
10,900 \\
7,150 \\
3,690\end{array}$ & $\begin{array}{l}17 \\
18 \\
19 \\
20 \\
21\end{array}$ & $\begin{array}{r}2,400 \\
12,800 \\
62,400 \\
18,200 \\
8,880\end{array}$ & $\begin{array}{l}22 \\
23 \\
24 \\
25 \\
26\end{array}$ & $\begin{array}{l}5,950 \\
4,890 \\
3,630 \\
2,880 \\
2,420\end{array}$ & $\begin{array}{l}27 \\
28 \\
29 \\
30 \\
31\end{array}$ & $\begin{array}{l}2,150 \\
1,900 \\
1,730 \\
1,630 \\
2,930\end{array}$ \\
\hline
\end{tabular}

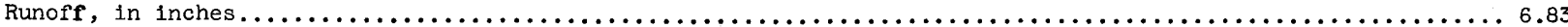

Gage height, in feet, and discharge, in cubic feet per second, at indicated time

\begin{tabular}{|c|c|c|c|c|c|c|c|c|c|c|c|}
\hline TIme & & $\begin{array}{c}\text { D18- } \\
\text { charge }\end{array}$ & Time & $\begin{array}{c}\text { Gage } \\
\text { helght }\end{array}$ & $\begin{array}{l}\text { Dis- } \\
\text { charge }\end{array}$ & Time & & & Time & & \\
\hline 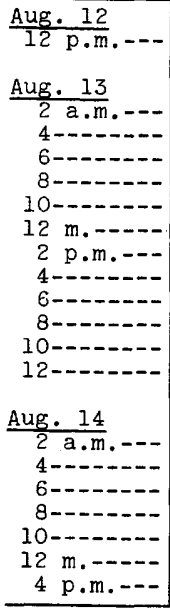 & $\begin{array}{l}2.12 \\
2.32 \\
2.69 \\
3.14 \\
3.49 \\
3.74 \\
3.91 \\
4.14 \\
4.49 \\
4.88 \\
5.35 \\
5.64\end{array}$ & $\begin{array}{r}525 \\
746 \\
1,280 \\
2,020 \\
2,710 \\
3,270 \\
3,670 \\
4,240 \\
5,170 \\
6,340 \\
7,980 \\
9,060\end{array}$ & 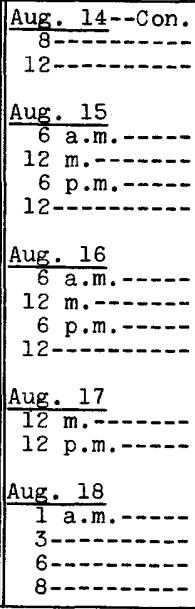 & $\begin{array}{l}4.10 \\
3.87 \\
3.70 \\
3.56\end{array}$ & $\begin{array}{l}4,140 \\
3,580 \\
3,180 \\
2,860\end{array}$ & 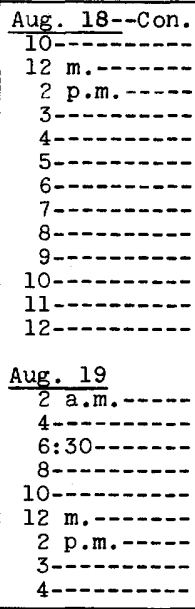 & $\begin{array}{r}3.90 \\
4.24 \\
4.64 \\
4.88 \\
5.58 \\
6.69 \\
7.75 \\
8.50 \\
9.51 \\
10.32 \\
11.55 \\
14.57 \\
16.55\end{array}$ & $\begin{array}{r}3,650 \\
4,490 \\
5,620 \\
6,340 \\
8,820 \\
13,500 \\
18,400 \\
22,100 \\
27,200 \\
31,800 \\
38,400 \\
56,300 \\
69,800 \\
\\
74,400 \\
76,500 \\
77,800 \\
77,100 \\
75,400 \\
72,600 \\
68,100 \\
62,800 \\
56,200\end{array}$ & 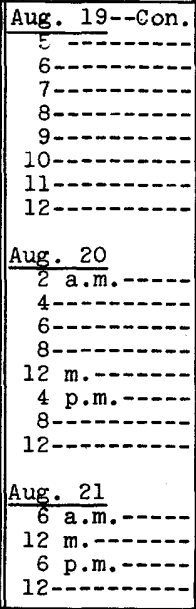 & $\begin{array}{l}13.60 \\
13.00 \\
12.35 \\
11.80 \\
11.33 \\
10.92 \\
10.46 \\
10.10\end{array}$ & $\begin{array}{l}50,500 \\
46,900 \\
43,000 \\
39,800 \\
37,200 \\
35,000 \\
32,400 \\
30,400\end{array}$ \\
\hline
\end{tabular}


(284) Lehigh River at Bethlehem, Pa.

Location.--Lat $40^{\circ} 37^{\prime} 05^{\prime \prime}$, long $75^{\circ} 21^{\prime} 55^{\prime \prime}$, on Ieft bank 1,650 ft upstream from Minsi Trail Bridge at Bethlehem, Northampton County, and 2,400 ft downstream from Monocacy Creek. Datum of gage is $208.45 \mathrm{ft}$ above mean sea level, datum of 1929.

Drainage area. $-1,279$ sq $\mathrm{mi}$.

Gage-height record.--Water-stage recorder graph except for period Aug. 19-24 when graph was drawn on basis of flood mark in gage shelter, engineer's staff gage readings, and wire-weight gage readings furnished by the U. S. Weather Bureau.

Discharge.--Stage-discharge relation defined by current-meter measurements below 50,000 cfs and extended to peak stage.

Shifting-control method used at times.

Maxima.--August 1955: Discharge, $91,300 \mathrm{cfs} 10$ a.m. Aug. 19 (gage height, 23.38 ft, from floodmark).

1902-05, 1909 to July 1955: Discharge, 92,000 cfs May 23, 1942 (gage height, 23.47 ft).

Flood of Feb. 28, 1902 reached a stage of $24.9 \mathrm{ft}$, from floodmark, site and datum in use in September 1902 (discharge about $88,000 \mathrm{cfs}$ ).

Mean discharge, in cubic feet per second, August 1955

\begin{tabular}{|c|c|c|c|c|c|c|c|c|c|c|c|}
\hline Day & $\mathrm{Cfs}$ & Day & $\mathrm{Cf}$ s & Day & Cfs & $\mathrm{Day}$ & Cfs & Day & Cfs & Day & Cfs \\
\hline $\begin{array}{l}1 \\
2 \\
3 \\
4 \\
5 \\
6\end{array}$ & $\begin{array}{l}463 \\
446 \\
436 \\
436 \\
441 \\
441\end{array}$ & $\begin{array}{r}7 \\
8 \\
9 \\
10 \\
11\end{array}$ & $\begin{array}{l}528 \\
633 \\
504 \\
468 \\
557\end{array}$ & $\begin{array}{l}12 \\
13 \\
14 \\
15 \\
16\end{array}$ & $\begin{array}{r}1,200 \\
4,500 \\
12,700 \\
9,670 \\
5,140\end{array}$ & $\begin{array}{l}17 \\
18 \\
19 \\
20 \\
21\end{array}$ & $\begin{array}{r}3,240 \\
9,890 \\
10,400 \\
22,800 \\
10,700\end{array}$ & $\begin{array}{l}22 \\
23 \\
24 \\
25 \\
26\end{array}$ & $\begin{array}{l}6,950 \\
4,870 \\
4,150 \\
3,750 \\
3,220\end{array}$ & $\begin{array}{l}27 \\
28 \\
29 \\
30 \\
31\end{array}$ & $\begin{array}{l}2,890 \\
2,630 \\
2,370 \\
2,250 \\
3,260\end{array}$ \\
\hline
\end{tabular}

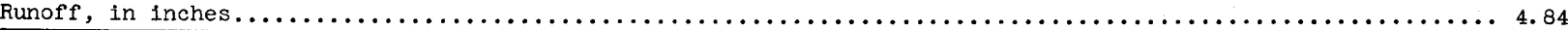

Gage height, in feet, and discharge, in cubic feet per second, at indicated time

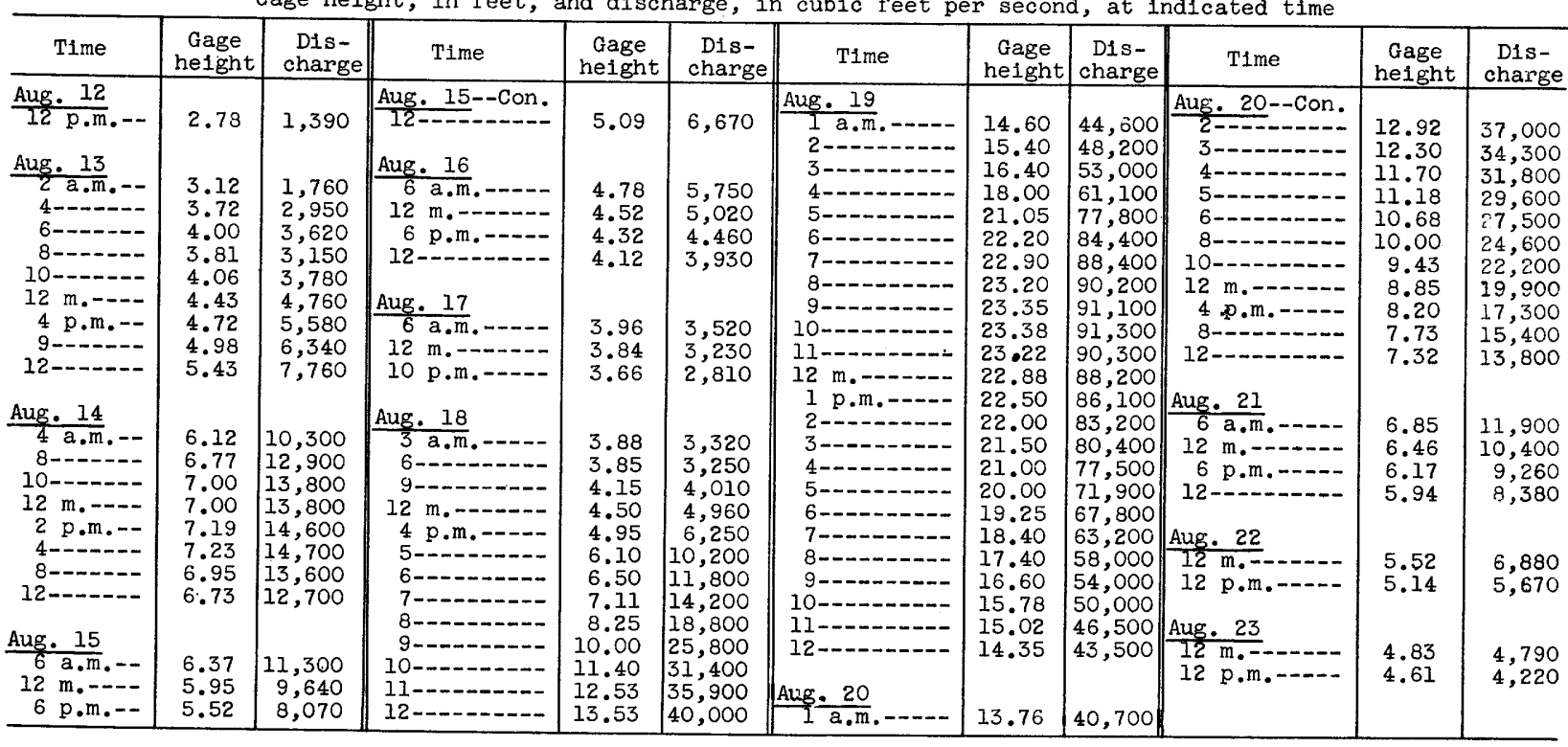


(287) Musconetcong River near Bloomsbury, N. J.

Location.--Lat $40^{\circ} 40^{\prime} 20^{\prime \prime}$, long $75^{\circ} 03^{\prime} 40^{\prime \prime}$, on right bank just downstream from highway bridge, $1 \frac{1}{2}$ miles upstream from Bloomsbury, Hunterdon County, and $9 \frac{1}{2}$ miles upstream from mouth. Datum of gage $1 \mathrm{~s} 274.83 \mathrm{ft}$ above mean sea level, datum of 1929 .

Drainage area. $--143 \mathrm{sq} \mathrm{mi}$.

Gage-helght record.--Water-stage recorder graph. Discharge record.--Stage-discharge relation defined by current-meter measurements below 1,800 cfs and extended to

peak stage on basis of slope-area determination.

Maxima.--August 1955: D1scharge, 4,430 cfs $9 \mathrm{a.m}$. Aug. 19 (gage height, 6.95 ft).

1921 to July 1955: Discharge, 5,760 efs (revised) Mar. 15, 1940 (gage height, 7.55 ft).

Remarks.--Flow regulated by Lake Hopatcong (usable capacity, $800,000,000 \mathrm{cu} f t$ ).

Difurnal fluctuation caused by small powerplants above station.

Mean discharge, in cublc feet per second, August 1955

\begin{tabular}{|c|c|c|c|c|c|c|c|c|c|c|c|}
\hline Day & $\mathrm{Cfs}$ & Day & $\mathrm{Cfs}$ & Day & Cfs & Day & $\mathrm{Crs}$ & Day & Cfs & Day & Cfs \\
\hline $\begin{array}{l}1 \\
2 \\
3 \\
4 \\
5 \\
6\end{array}$ & $\begin{array}{l}40 \\
35 \\
31 \\
32 \\
39 \\
32\end{array}$ & $\begin{array}{r}7 \\
8 \\
9 \\
10 \\
11\end{array}$ & $\begin{array}{l}21 \\
48 \\
44 \\
46 \\
48\end{array}$ & $\begin{array}{l}12 \\
13 \\
14 \\
15 \\
16\end{array}$ & $\begin{array}{l}132 \\
858 \\
674 \\
476 \\
385\end{array}$ & $\begin{array}{l}17 \\
18 \\
19 \\
20 \\
21\end{array}$ & $\begin{array}{r}335 \\
630 \\
2,890 \\
2,390 \\
1,720\end{array}$ & $\begin{array}{l}22 \\
23 \\
24 \\
25 \\
26\end{array}$ & $\begin{array}{r}1,250 \\
928 \\
734 \\
594 \\
500\end{array}$ & $\begin{array}{l}27 \\
28 \\
29 \\
30 \\
31\end{array}$ & $\begin{array}{l}451 \\
376 \\
342 \\
305 \\
290\end{array}$ \\
\hline
\end{tabular}

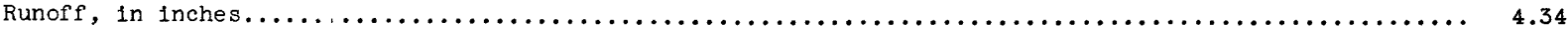

Gage helght, in feet, and discharge in cubic feet per second, at indicated time

\begin{tabular}{|c|c|c|c|c|c|c|c|c|c|c|c|}
\hline Time & $\begin{array}{c}\text { Gage } \\
\text { he1ght }\end{array}$ & $\begin{array}{c}\text { Dis- } \\
\text { charge }\end{array}$ & Time & $\begin{array}{c}\text { Gage } \\
\text { he1ght }\end{array}$ & $\begin{array}{l}\text { Dis- } \\
\text { charge }\end{array}$ & Time & & $\begin{array}{c}\text { Dis- } \\
\text { charge }\end{array}$ & Time & & $\begin{array}{c}\text { Dis- } \\
\text { charge }\end{array}$ \\
\hline 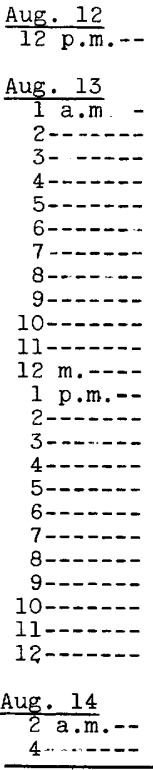 & $\begin{array}{l}1.7 \\
1.73 \\
1.90 \\
2.35 \\
2.77 \\
2.95 \\
3.21 \\
3.34 \\
3.50 \\
4.00 \\
4.21 \\
4.20 \\
4.15 \\
4.12 \\
4.06 \\
3.98 \\
3.85 \\
3.68 \\
3.45 \\
3.23 \\
3.05 \\
2.93 \\
2.85 \\
2.82\end{array}$ & $\begin{array}{r}187 \\
207 \\
265 \\
435 \\
612 \\
692 \\
815 \\
880 \\
960 \\
1,240 \\
1,360 \\
1,350 \\
1,320 \\
1,300 \\
1,270 \\
1,230 \\
1,160 \\
1,060 \\
935 \\
825 \\
738 \\
684 \\
648 \\
634\end{array}$ & 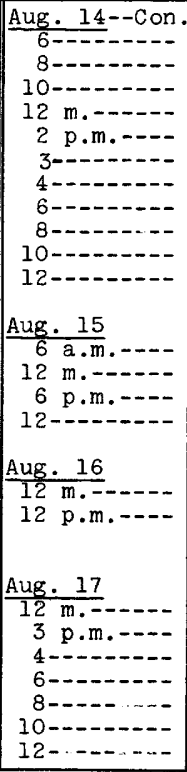 & $\begin{array}{l}2.85 \\
2.88 \\
2.83 \\
2.80 \\
2.98 \\
3.30 \\
3.35 \\
3.06 \\
2.91 \\
2.75 \\
2.65\end{array}$ & $\begin{array}{l}648 \\
661 \\
638 \\
625 \\
706 \\
860 \\
885 \\
742 \\
674 \\
602 \\
560\end{array}$ & 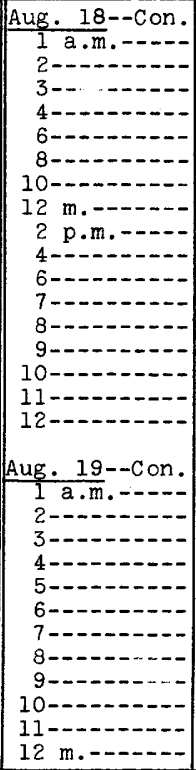 & $\begin{array}{l}2.41 \\
2.65 \\
2.80 \\
2.81 \\
2.66 \\
2.56 \\
2.46 \\
2.45 \\
2.51 \\
2.49 \\
2.48 \\
2.50 \\
2.90 \\
3.45 \\
3.90 \\
4.35 \\
4.65\end{array}$ & $\begin{array}{r}459 \\
560 \\
625 \\
630 \\
564 \\
522 \\
479 \\
475 \\
500 \\
491 \\
487 \\
495 \\
670 \\
935 \\
1,180 \\
1,440 \\
1,620 \\
\\
\\
1,820 \\
2,060 \\
2,440 \\
2,990 \\
3,430 \\
3,610 \\
3,720 \\
4,260 \\
4,430 \\
4,060 \\
3,510 \\
3,100\end{array}$ & 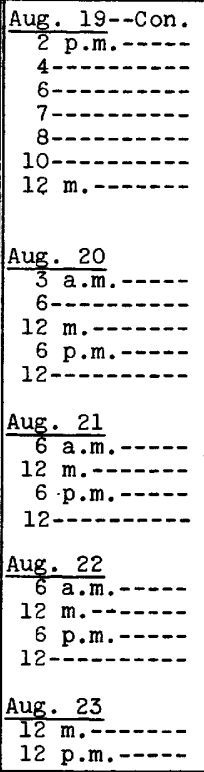 & $\begin{array}{l}5.85 \\
5.79 \\
5.62 \\
5.43 \\
5.21\end{array}$ & $\begin{array}{l}2,660 \\
2,590 \\
2,410 \\
2,220 \\
2,020 \\
\\
1,870 \\
1,730 \\
1,560 \\
1,450\end{array}$ \\
\hline
\end{tabular}


(288) Delaware River at Riegelsville, N. J.

Location.--Lat $40^{\circ} 35^{\prime} 36^{\prime \prime}$, long $75^{\circ} 11^{\prime} 17^{\prime \prime}$, on left bank $20 \mathrm{ft}$ upstream from suspension bridge at Riegelsville, Warren County, and $600 \mathrm{ft}$ upstream from Musconetcong River. Records include flow of Musconetcong River. Datum of gage is $125.12 \mathrm{ft}$ above mean sea level, datum of 1929 .

Drainage area.--6,328 sq mi (includes that of Musconetcong River).

Gag-helght record.--Water-stage recorder graph except Aug. 19 (12 m.) to Aug. 25 (11 a.m.) for which graph was drawn on basis of hourly readings by New Jersey Power \& Light Company 3.5 miles downstream and floodmark.

Discharge record.--Stage-discharge relation defined by current-meter measurements below 160,000 cfs. Discharge records being held pending completion of a basin study

Maxima.-August 1955: Gage he1ght, 38.85 (from floodmark) 1 a.m. Aug. 20.

1906 to July 1955: Discharge, $210,000 \mathrm{cfs}$ Mar. 19, 1936 (gage helght, $32.45 \mathrm{ft}$ ).

Flood of Oct. 10, 1903, reached a stage of $35.9 \mathrm{ft}$, from floodmarks (discharge, about 275,000 cfs).

Remarks.--Diversion above station to Delaware Division Canal. Flow regulated by Lakes Hopatcong and Wallenpaupack and by Pepacton, Toronto, Cliff Lake, Swinging Bridge, and Neversink Reservoirs (combined capacity, $37,732,000,000 \mathrm{cu} f \mathrm{t}$ ).

Gage height, in feet, and discharge, in cublc feet per second, at indicated time

\begin{tabular}{|c|c|c|c|c|c|c|c|c|c|c|c|}
\hline TIme & $\begin{array}{c}\text { Gage } \\
\text { helght }\end{array}$ & $\begin{array}{l}\text { * Dis- } \\
\text { charge }\end{array}$ & Time & $\begin{array}{c}\text { Gage } \\
\text { he1ght }\end{array}$ & $\begin{array}{l}\text { *Dis- } \\
\text { charge }\end{array}$ & Time & $\begin{array}{c}\text { Gage } \\
\text { he1ght }\end{array}$ & $\begin{array}{l}\text { *Dis- } \\
\text { charge }\end{array}$ & Time & $\begin{array}{c}\text { Gage } \\
\text { height }\end{array}$ & $\begin{array}{l}\text { *D1s- } \\
\text { charge }\end{array}$ \\
\hline 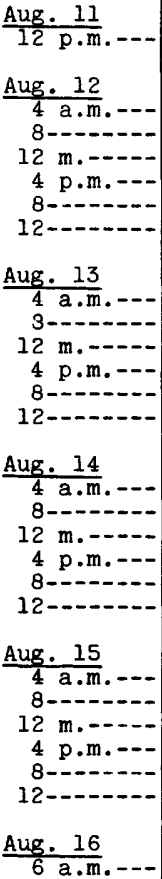 & $\begin{array}{r}3.56 \\
4.80 \\
5.56 \\
6.00 \\
6.40 \\
7.26 \\
\\
7.72 \\
8.75 \\
10.22 \\
10.76 \\
10.65 \\
10.17\end{array}$ & & 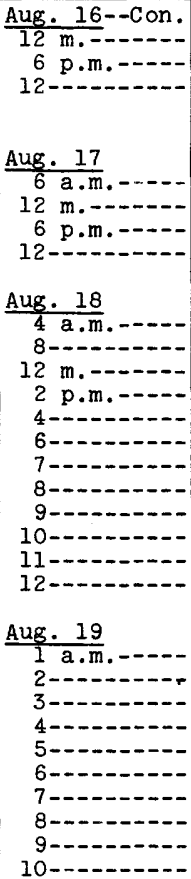 & $\begin{array}{r}7.54 \\
6.93 \\
6.41 \\
\\
\\
6.00 \\
5.76 \\
5.56 \\
5.28 \\
\\
5.31 \\
5.51 \\
5.62 \\
5.78 \\
6.04 \\
6.45 \\
7.00 \\
7.90 \\
8.65 \\
9.30 \\
10.25 \\
11.50 \\
\\
12.90 \\
14.20 \\
16.00 \\
19.30 \\
22.10 \\
24.70 \\
26.70 \\
28.50 \\
29.80 \\
30.90\end{array}$ & & $\begin{array}{r}\text { Aug. } 19 \\
112 \mathrm{~m} . \\
1 \mathrm{p} . \mathrm{m} . \\
2 \\
3 \\
4 \\
5 \\
6 \\
7 \\
8 \\
9\end{array}$ & $\begin{array}{l}32.00 \\
32.7 \\
33.2 \\
34.0 \\
34.8 \\
35.6 \\
36.3 \\
37.0 \\
37.5 \\
37.9 \\
38.2 \\
38.4 \\
38.6 \\
38.7 \\
\\
\\
38.85 \\
38.8 \\
38.5 \\
38.2 \\
37.7 \\
37.2 \\
36.6 \\
35.9 \\
35.0 \\
34.2 \\
33.7 \\
33.2 \\
31.5 \\
30.0 \\
29.0 \\
28.0 \\
27.0 \\
26.0 \\
25.0\end{array}$ & & 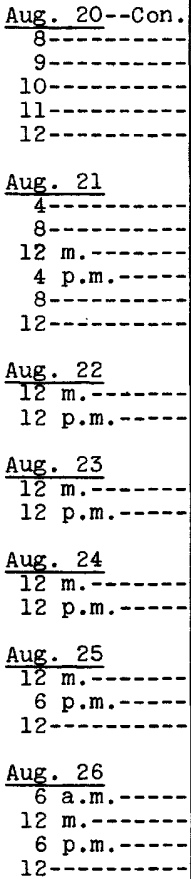 & $\begin{array}{r}24.2 \\
23.5 \\
22.9 \\
22.4 \\
22.0 \\
\\
20.0 \\
18.4 \\
17.2 \\
16.2 \\
15.5 \\
15.0 \\
\\
13.6 \\
12.6 \\
\\
11.8 \\
11.4 \\
\\
10.4 \\
9.2 \\
8.32 \\
8.04 \\
7.74 \\
7.43 \\
7.17 \\
6.97 \\
6.78\end{array}$ & \\
\hline
\end{tabular}

* Discharge records being held pending completion of a basin study. 
(289) Tohickon Creek near Pipersville, Pa.

Location.--Lat $10^{\circ} 26^{\prime} 00^{\prime \prime}$, long $75^{\circ} 07^{\prime} 00^{\prime \prime}$, on right bank at highway bridge, 1.5 miles northeast of Pipersville, Bucks count, and 4.5 miles upstream from mouth. Datum of gage is $258.96 \mathrm{ft}$ above mean sea level, datum of 1929 (tencount, and $4.5 \mathrm{miles}$

tative adjustment).

Gage-height record.--Water-stage recorder graph.

Discharge record.-Stage-discharge relation defined by current-meter measurements below 3,600 cfs and extended to peak stage on basis of slope-area determination at gage height $10.48 \mathrm{ft}$. Shifting-control method at times. Maxima.--Ausust 1955: Discharge, 16,000 ofs $12 \mathrm{p.m}$. Aug. 18 (gage height, $11.26 \mathrm{ft}$ ).

1935 to July 1955: Discharge, $13,700 \mathrm{cfs}$ Aug. 9, 1942 (gage height, 10.48 ft), on bas1s of slope-area determination.

\begin{tabular}{|c|c|c|c|c|c|c|c|c|c|c|c|}
\hline Day & Cfs & Day & Cfs & Day & Cfs & Day & Cfs & Day & Cf's & Day & Cfs \\
\hline $\begin{array}{l}1 \\
2 \\
3 \\
4 \\
5 \\
6\end{array}$ & $\begin{array}{r}0.5 \\
.4 \\
.5 \\
.5 \\
.5 \\
.5\end{array}$ & $\begin{array}{r}7 \\
8 \\
9 \\
10 \\
11\end{array}$ & $\begin{array}{l}0.5 \\
2.7 \\
4.2 \\
5.5 \\
5.5\end{array}$ & $\begin{array}{l}12 \\
13 \\
14 \\
15 \\
16\end{array}$ & $\begin{array}{r}166 \\
6,090 \\
1,650 \\
303 \\
128\end{array}$ & $\begin{array}{l}17 \\
18 \\
19 \\
20 \\
21\end{array}$ & $\begin{array}{r}73 \\
2,350 \\
3,740 \\
326 \\
127\end{array}$ & $\begin{array}{l}22 \\
23 \\
24 \\
25 \\
26\end{array}$ & $\begin{array}{r}553 \\
170 \\
78 \\
52 \\
38\end{array}$ & $\begin{array}{l}27 \\
28 \\
29 \\
30 \\
31\end{array}$ & $\begin{array}{l}31 \\
24 \\
18 \\
17 \\
19\end{array}$ \\
\hline
\end{tabular}

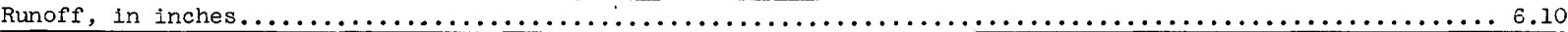

Gage height, in feet, and discharge, in cubic feet per second, at indicated time

\begin{tabular}{|c|c|c|c|c|c|c|c|c|c|c|c|}
\hline Time & $\begin{array}{c}\text { Gage } \\
\text { height }\end{array}$ & $\begin{array}{l}\text { Dis- } \\
\text { charge }\end{array}$ & Time & $\begin{array}{c}\text { Gage } \\
\text { height }\end{array}$ & $\begin{array}{l}\text { D1s- } \\
\text { charge }\end{array}$ & Time & $\begin{array}{c}\text { Gage } \\
\text { he1ght }\end{array}$ & $\begin{array}{l}\text { Dis- } \\
\text { charge }\end{array}$ & Time & $\begin{array}{c}\text { Gage } \\
\text { he1ght }\end{array}$ & $\begin{array}{l}\text { Dis- } \\
\text { charge }\end{array}$ \\
\hline 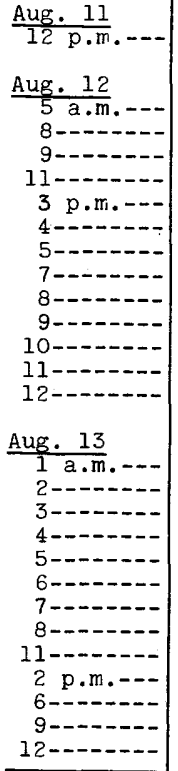 & $\begin{array}{l}4.19 \\
4.55 \\
5.48 \\
6.61 \\
7.30 \\
8.18 \\
7.99 \\
8.12 \\
8.61 \\
8.03 \\
7.44 \\
6.98 \\
6.43\end{array}$ & $\begin{array}{l}1,570 \\
1,930 \\
3,050 \\
4,800 \\
6,020 \\
7,810 \\
7,400 \\
7,670 \\
8,780 \\
7,480 \\
6,290 \\
5,430 \\
4,500\end{array}$ & 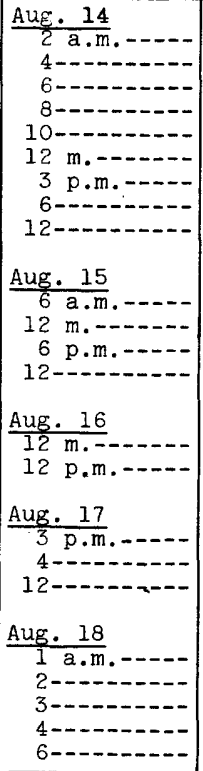 & $\begin{array}{l}5.96 \\
5.47 \\
4.97 \\
4.51 \\
4.11 \\
3.73 \\
3.37 \\
3.05 \\
2.80 \\
\\
2.63 \\
2.45 \\
2.28 \\
2.14\end{array}$ & $\begin{array}{r}3,750 \\
3,040 \\
2,390 \\
1,890 \\
1,500 \\
1,170 \\
888 \\
648 \\
474 \\
\\
374 \\
288 \\
223 \\
179 \\
\\
123 \\
86 \\
\\
65 \\
70 \\
74\end{array}$ & 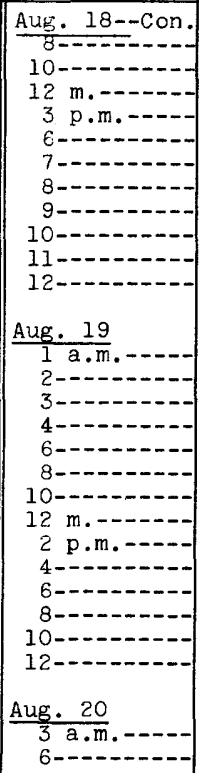 & $\begin{array}{r}10.73 \\
9.68 \\
8.55 \\
7.53 \\
6.27 \\
5.61 \\
5.15 \\
4.76 \\
4.37 \\
4.06 \\
3.80 \\
3.52 \\
3.25 \\
3.06\end{array}$ & $\begin{array}{r}14,400 \\
11,400 \\
8,640 \\
6,460 \\
4,240 \\
3,230 \\
2,620 \\
2,160 \\
1,750 \\
1,450 \\
1,230 \\
1,010 \\
798 \\
655\end{array}$ & 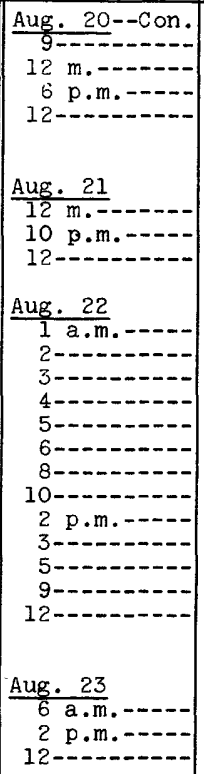 & $\begin{array}{l}3.30 \\
4.17 \\
4.30 \\
3.87 \\
3.38 \\
3.03 \\
2.74 \\
2.57 \\
2.42 \\
2.53 \\
2.72 \\
2.58 \\
2.44\end{array}$ & $\begin{array}{r}835 \\
1,550 \\
1,680 \\
1,290 \\
895 \\
632 \\
437 \\
343 \\
275 \\
324 \\
425 \\
348 \\
284\end{array}$ \\
\hline
\end{tabular}


(290) Delaware River at Trenton, N. J.

Location.--Lat $40^{\circ} 13118^{\prime \prime}$, long $74^{\circ} 46^{\prime} 38^{\prime \prime}$ on left bank $450 \mathrm{ft}$ upstream from Calhoun Street bridge at Trenton, Mercer County, and half a mile upstream from Assunpink Creek. Datum of gage is $7.77 \mathrm{ft}$ above mean sea level, datum of 1929.

Drainage area. $--6,780 \mathrm{so} \mathrm{ml}$.

Gage-height record.--Water-stage recorder graph except for Aug. 19 (10 a.m.) to Aug. 23 (1 p.m.) for which graph was drawn based on hourly wire-weight gage readings by Delaware River Joint Toll Bridge Commission and high-water mark in gage house.

Discharge record.--Stage-discharge relation defined by current-meter measurements to 230,000 cfs and extended to peak stage by logarithmic plotting.

Maxima.--August 1955: Discharge, about 329,000 cfs 6-7 a.m. Aug. 20 (gage he1ght, $20.83 \mathrm{ft}$, from high-water mark gage house).

1913 to July 1955: Discharge, $227,000 \mathrm{cfs}$ Mar. 19, 1936 (gage height, $16.66 \mathrm{ft}$ ).

Maxlmum stage known, 22.8 ft Mar. 8, 1904, from floodmark (1ce Jam). Flood of Oct. 11, 1903, reached a stage of about $20.7 \mathrm{ft}$ (discharge estimated, $295,000 \mathrm{cfs}$ ).

Remerk and by Pepacton, Toronto, Cliff Lake, Swinging Bridge, and Neversink Reservoirs (combined capacity, 37,732,000,000 cu $\mathrm{ft}$ ).

Mean discharge, in cubic feet per second, Augusi 1955

\begin{tabular}{c|c||c|c||c|c||c|c||c|c|c||c}
\hline Day & Cfs & Day & Cfs & Day & Uf's & Day & Cfs & Day & Cfs & Day & Cfs \\
\hline 1 & 1,900 & 7 & 2,340 & 12 & 3,870 & 17 & 15,600 & 22 & 50,700 & 27 & 17,000 \\
$\tau$ & 1,900 & 8 & 2,880 & 13 & 28,500 & 18 & 16,900 & 23 & 37,800 & 28 & 14,600 \\
3 & 1,620 & 9 & 2,480 & 14 & 28,400 & 19 & 163,000 & 24 & 33,800 & 29 & 12,600 \\
4 & 1,730 & 10 & 1,920 & 15 & 33,300 & 20 & 281,000 & 25 & 26,000 & 30 & 11,600 \\
5 & 2,220 & 11 & 2,120 & 16 & 23,500 & 21 & 88,100 & 26 & 20,500 & 31 & 11,000 \\
6 & 2,160 & & & & & & & & & & \\
\hline
\end{tabular}

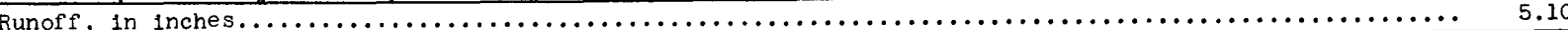

Gage height, in feet, and discharge, in cubic feet per second, at indicated time

\begin{tabular}{|c|c|c|c|c|c|c|c|c|c|c|c|}
\hline Time & $\begin{array}{l}\text { Gage } \\
\text { elght }\end{array}$ & $\begin{array}{c}\text { Dis- } \\
\text { charge }\end{array}$ & Time & $\begin{array}{c}\text { Gage } \\
\text { helght }\end{array}$ & $\begin{array}{l}\text { Dis- } \\
\text { charge }\end{array}$ & Time & & $\begin{array}{l}\text { D1s- } \\
\text { charge }\end{array}$ & Time & & $\begin{array}{l}\text { Dis- } \\
\text { charge }\end{array}$ \\
\hline 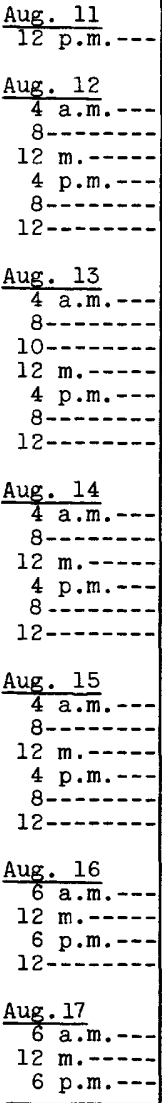 & $\begin{array}{l}2.30 \\
5.02 \\
5.61 \\
5.53 \\
5.05 \\
4.30 \\
3.91\end{array}$ & $\begin{array}{l}12,500 \\
35,300 \\
41,700 \\
40,800 \\
35,600 \\
28,100 \\
24,600\end{array}$ & 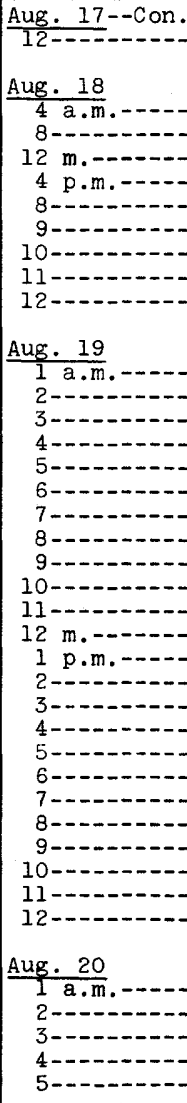 & $\begin{array}{r}9.05 \\
9.97 \\
10.58 \\
10.64 \\
10.15 \\
9.64 \\
9.34 \\
9.45 \\
9.82 \\
11.0 \\
12.0 \\
12.7 \\
13.7 \\
14.3 \\
14.9 \\
15.4 \\
15.9 \\
16.4 \\
17.0 \\
17.4 \\
17.8 \\
18.3 \\
18.8 \\
19.1 \\
\end{array}$ & $\begin{array}{r}86,600 \\
101,000 \\
111,000 \\
111,000 \\
104,000 \\
95,400 \\
90,900 \\
92,600 \\
98,100 \\
117,000 \\
134,000 \\
147,000 \\
165,000 \\
177,000 \\
189,000 \\
200,000 \\
210,000 \\
221,000 \\
235,000 \\
245,000 \\
254,000 \\
266,000 \\
278,000 \\
285,000\end{array}$ & 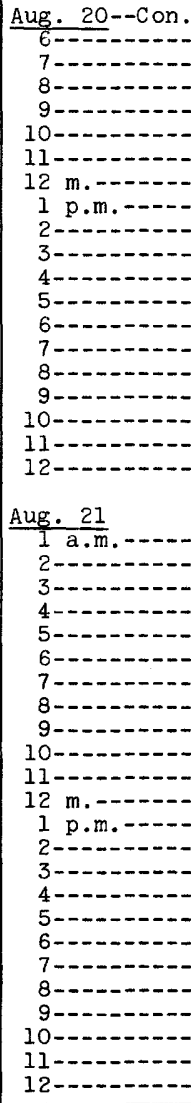 & $\begin{array}{r}12.5 \\
11.9 \\
11.3 \\
11.0 \\
10.6 \\
10.2 \\
9.9 \\
9.6 \\
9.4 \\
9.0 \\
8.7 \\
8.5 \\
8.4 \\
8.2 \\
8.1 \\
7.9 \\
7.8 \\
7.7 \\
7.6 \\
7.4 \\
7.3 \\
7.2 \\
7.2 \\
7.2\end{array}$ & $\begin{array}{r}143,000 \\
132,000 \\
122,000 \\
117,000 \\
111,000 \\
104,000 \\
99,400 \\
94,800 \\
91,800 \\
85,800 \\
81,600 \\
78,800 \\
77,400 \\
74,600 \\
73,200 \\
70,400 \\
69,000 \\
67,600 \\
66,300 \\
63,700 \\
62,400 \\
61,100 \\
61,100 \\
61,100\end{array}$ & $\begin{array}{r}\text { Aug. } 22 \\
4 \mathrm{a} \cdot \mathrm{m} . \\
8 \mathrm{~m} \\
12 \mathrm{~m} . \mathrm{m} \\
4 \mathrm{p} \cdot \mathrm{m} . \\
8 \\
12\end{array}$ & $\begin{array}{l}4.25 \\
4.04 \\
3.86 \\
3.71 \\
\\
3.40 \\
3.13\end{array}$ & $\begin{array}{l}59,800 \\
53,300 \\
49,700 \\
46,100 \\
43,800 \\
41,600\end{array}$ \\
\hline
\end{tabular}


(291) Neshaminy Creek near Langhorne, Pa.

Location.--Lat $40^{\circ} 10^{\prime} 25^{\prime \prime}$, long $74^{\circ} 57^{\prime} 30^{\prime \prime}$, on left bank at bridge on State Highway $213,0.3$ mile downstream from Mill Creek and 1.7 miles west of Langhorne, Bucks County. Datum of gage is $40.57 \mathrm{ft}$ above mean sea level, datum of 1929 , supplementary adjustment of 1943.

Drainage area. $--210 \mathrm{sq} \mathrm{mi}$.

Gage-height record. - Water-stage recorder graph except for period Aug. 19-22 which was reconstructed on basis of records for nearby stations and floodmark of peak.

Discharge record.--Stage-discharge relation defined by current-meter measurements below 4,700 cfs and extended above on basis of contracted-opening determination at $15.94 \mathrm{ft}$ and slope-area determination at $22.84 \mathrm{ft}$.

Maxima.--Aukust 1955: Discharge, 49,300 ofs about 9 a.m. Aug. 19 (gage height, 22.84 ft, from floodmark).

1934 to July 1955: Discharge, 24,800 cfs July 23, 1938 (gage height, $15.94 \mathrm{ft}$ ) by contracted-opening method.

Flood of Aug. 23, 1933 reached a stage of $17.3 \mathrm{ft}$ (discharge, 30,000 cfs, from rating curve extended as explained above).

Remarks.--Occasional regulation by Springfield Lake (capacity, 650 million gallons); no significant regulation during flood period.

\begin{tabular}{|c|c|c|c|c|c|c|c|c|c|c|c|}
\hline Day & Cfs & Day & Cfs & Day & Cfs & Day & Cfs & Day & Cfs & Day & Cfs \\
\hline 1 & 13 & 7 & 17 & 12 & 550 & 17 & 213 & 22 & 1,910 & 27 & 253 \\
\hline 2 & 11 & 8 & 73 & 13 & 12,300 & 18 & 1,290 & 23 & 620 & 28 & 233 \\
\hline 3 & 13 & 9 & 123 & 14 & 1,770 & 19 & 27,300 & 24 & 415 & 29 & 205 \\
\hline 4 & 18 & 10 & 40 & 15 & 479 & 20 & 3,230 & 25 & 341 & 30 & 198 \\
\hline $\begin{array}{l}5 \\
6\end{array}$ & $\begin{array}{l}18 \\
18\end{array}$ & 11 & 46 & 16 & 276 & 21 & 554 & 26 & 290 & 31 & 198 \\
\hline
\end{tabular}

Gage height, in feet, and discharge, in cubic feet per second, at indicated time

\begin{tabular}{|c|c|c|c|c|c|c|c|c|c|c|c|}
\hline Time & $\begin{array}{c}\text { Gage } \\
\text { height }\end{array}$ & $\begin{array}{l}\text { Dis- } \\
\text { charge }\end{array}$ & Time & $\begin{array}{c}\text { Gage } \\
\text { height }\end{array}$ & \begin{tabular}{|} 
Dis- \\
charge
\end{tabular} & Time & $\begin{array}{c}\text { Gage } \\
\text { helght }\end{array}$ & $\begin{array}{c}\text { Dis- } \\
\text { charge }\end{array}$ & Time & $\begin{array}{c}\text { Gage } \\
\text { height }\end{array}$ & $\begin{array}{l}\text { Dis- } \\
\text { charge }\end{array}$ \\
\hline$\frac{\text { Aug. } 11}{12 \text { p.m. }}$ & 0.93 & 43 & \begin{tabular}{|l} 
Aug. $13--C o n$ \\
6 \\
10 \\
$12-$
\end{tabular} & $\begin{array}{l}13.55 \\
12.42 \\
10.40\end{array}$ & $\begin{array}{l}17,300 \\
14,600 \\
10,200\end{array}$ & Aug. 17--Con. & $\begin{array}{l}1.46 \\
1.51\end{array}$ & $\begin{array}{l}194 \\
213\end{array}$ & Aug. 19--Con. & $\begin{array}{r}12.60 \\
10.40 \\
8.80\end{array}$ & $\begin{array}{r}15,000 \\
10,200 \\
7,330\end{array}$ \\
\hline$\frac{\text { Aug. } 12}{4 \mathrm{a} \cdot \mathrm{m} .-}$ & & & Aug. 14 & & & Aug. 18 & & & Aug. 20 & & \\
\hline $\begin{array}{r}7--0- \\
8-0- \\
10-\end{array}$ & $\begin{array}{r}.92 \\
1.12 \\
1.07\end{array}$ & $\begin{array}{l}34 \\
83 \\
71\end{array}$ & $\begin{array}{l}1 \text { a.m. } \\
2-1 \\
3\end{array}$ & $\begin{array}{l}8.45 \\
6.37 \\
5.35\end{array}$ & $\begin{array}{l}6,780 \\
4,060 \\
3,000\end{array}$ & $\begin{array}{l}2 \text { a.m. } \\
9-1\end{array}$ & $\begin{array}{l}1.66 \\
1.93 \\
2.15\end{array}$ & $\begin{array}{l}274 \\
388 \\
489\end{array}$ & $\begin{array}{r}6 \text { a.m... } \\
12 \text { m. } \\
6\end{array}$ & $\begin{array}{l}6.60 \\
5.06 \\
3.93\end{array}$ & $\begin{array}{l}4,310 \\
2,720 \\
1,720\end{array}$ \\
\hline $\begin{aligned} 12 & \text { m.-- } \\
1 & \text { p.m.-- }\end{aligned}$ & & $\begin{array}{r}65 \\
712\end{array}$ & $\begin{array}{l}4-0- \\
8-\cdots\end{array}$ & $\begin{array}{l}4.63 \\
3.47\end{array}$ & $\begin{array}{l}2,340 \\
1,360\end{array}$ & 6 p.m.- & $\begin{array}{l}2.08 \\
2.33\end{array}$ & & $12-10$ & $\begin{array}{l}3.95 \\
3.00\end{array}$ & 1, 990 \\
\hline 6 & 2.78 & 842 & 2 p.m.- & 2.80 & 855 & & 3.11 & 1,070 & Aug. 21 & & \\
\hline $\begin{array}{l}6--1-1- \\
8-\cdots-1-\end{array}$ & $\begin{array}{l}2.59 \\
2.83 \\
3.13\end{array}$ & $\begin{array}{r}724 \\
874 \\
\end{array}$ & $12-0$ & $\begin{array}{l}2.99 \\
2.42\end{array}$ & $\begin{array}{l}983 \\
626\end{array}$ & $10-19-1$ & $\begin{array}{l}4.68 \\
7.65 \\
9.5 ?\end{array}$ & $\begin{array}{l}2,380 \\
5,630 \\
8,550\end{array}$ & 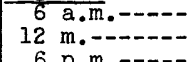 & $\begin{array}{l}2.25 \\
1.80\end{array}$ & $\begin{array}{l}538 \\
332 \\
375\end{array}$ \\
\hline $10 \ldots$ & 3.96 & 1,750 & Aug. 15 & & & $12=$ & 10.77 & 11,000 & $12-0-0$ & $\begin{array}{l}1.00 \\
2.95\end{array}$ & 955 \\
\hline $12-\ldots$ & 4.85 & 2,540 & $12 \mathrm{~m} \cdot$ & 2.12 & 475 & & & & & & \\
\hline Aug. 13 & & & $12 \mathrm{p.m}$. & & 341 & $\frac{\text { Aug. } 19}{1 \mathrm{a} \cdot \mathrm{m}}$ & 12.00 & 13,600 & $\frac{\text { Aug. } 22}{2}$ a.m. & 3.70 & 1,540 \\
\hline 3 a.m.-- & 5.95 & 3,600 & & & & $2 \cdots$ & 14.20 & 18,900 & $6---1-1--$ & & \\
\hline $5-\ldots$ & $\begin{array}{l}7.90 \\
9.54\end{array}$ & $\begin{array}{l}5,980 \\
8,580\end{array}$ & $\frac{\text { Aug. } 16}{12 \mathrm{~m} \cdot-}$ & 65 & 270 & $6-\ldots$ & 18.32 & 31,500 & 2 & 5.20 & $\begin{array}{l}2,850 \\
2,310\end{array}$ \\
\hline $6---\cdots$ & 10.48 & 10,400 & 12 p.m.- & 1.54 & 225 & $9--\cdots$ & 22.84 & & 4 & & 1,840 \\
\hline $8------$ & & 13,2 & & & & $12 \mathrm{~m}$. & 21.30 & & $6-\cdots$ & & 1,400 \\
\hline $11-\ldots$ & 12.75 & & Aug. 17 & & & & & & 10 & & 990 \\
\hline 2 p.m. & 12.61 & 15,0 & $12 \mathrm{mo}$ & 1.52 & 217 & & 14.80 & & $12 \ldots$ & 2.85 & 888 \\
\hline
\end{tabular}

(294) Little Schuylkill River at Tamaqua, Pa.

Location.--Lat $40^{\circ} 48^{\prime} 20^{\prime \prime}$, long $75^{\circ} 58^{\prime} 20^{\prime \prime}$, on left bank at pumping plant of Panther Valley Water Co., 0.6 mile upstream from Tamaqua, Schuylkill County, and 0.8 m1le upstream from Panther Creek. Datum of gage is $817.48 \mathrm{ft}$ above mean sea leve1, datum of 1929 , supplementary adjustment of 1943

Drainage area $--42.9 \mathrm{sq} \mathrm{mi}$.

Gage-height record. --water-stage recorder graph.

Discharge record.--Stage-discharge relation defined by current-meter measurements below $3,200 \mathrm{cfs}$ and extended above on basis of contracted-opening determination at peak stage.

Maxima.--August 1955: D1scharge, 7,790 cfs 5:30 p.m. Aug. 18 (gage height, $11.10 \mathrm{ft}$ ).

1916 to July 1955: Discharge, 4,310 cfs May 22, 1942 (gage height, $7.95 \mathrm{ft}$ )

Remarks. - Figures of daily discharge and monthly mean do not include water diverted from Still creek Reservolr (capacity, $133,900,000 \mathrm{cu} f \mathrm{t}$ ).

Mean discharge, in cub1c feet per second, August 1955

\begin{tabular}{c|c||c|c||c|c||c|c||c|c||c|c}
\hline Day & Cfs & Day & Cfs & Day & Cfs & Day & Cfs & Day & Cfs & Day & Cfs \\
\hline 1 & 10.5 & 7 & 12 & 12 & 6.1 & 17 & 38 & 22 & 328 & 27 & 112 \\
$\frac{6}{3}$ & 10 & 8 & 11.5 & 13 & 187 & 18 & 2,080 & 23 & 324 & 28 & 107 \\
4 & 9.5 & 9 & 9.8 & 14 & 91 & 19 & 1,600 & 24 & 200 & 29 & 105 \\
5 & 10 & 10 & 9.8 & 15 & 59 & 20 & 577 & 25 & 172 & 30 & 99 \\
6 & 10.5 & 11 & 23 & 16 & 42 & 21 & 344 & 26 & 137 & 31 & 166 \\
\hline
\end{tabular}

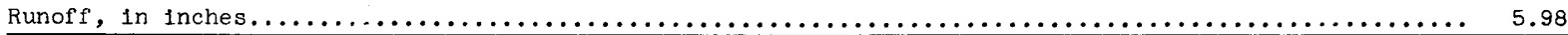


FLOOD DATA

(294) Ilttle Schuylk1li River at Tamaqua, Pa.--Continued

Gage he1ght, in feet, and discharge, in cubic feet per second, at indicated time

\begin{tabular}{|c|c|c|c|c|c|c|c|c|c|c|c|}
\hline Time & $\begin{array}{c}\text { Gage } \\
\text { he1ght }\end{array}$ & $\begin{array}{l}\text { Dis- } \\
\text { charge }\end{array}$ & Time & $\begin{array}{c}\text { Gage } \\
\text { he1ght }\end{array}$ & $\begin{array}{l}\text { Dis- } \\
\text { charge }\end{array}$ & Time & $\begin{array}{c}\text { Gage } \\
\text { helght }\end{array}$ & $\begin{array}{l}\text { Dis- } \\
\text { charge }\end{array}$ & Time & $\begin{array}{c}\text { Gage } \\
\text { he1ght }\end{array}$ & $\begin{array}{l}\text { D1s- } \\
\text { charge }\end{array}$ \\
\hline 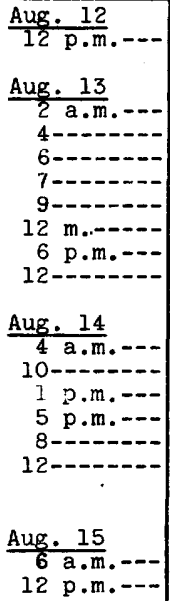 & $\begin{array}{l}2.12 \\
2.40 \\
2.60 \\
2.83 \\
2.92 \\
2.93 \\
3.19 \\
2.84\end{array}$ & $\begin{array}{r}35 \\
75 \\
116 \\
177 \\
206 \\
209 \\
300 \\
180\end{array}$ & 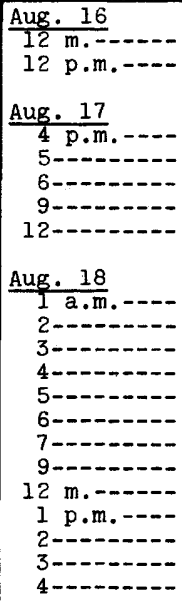 & $\begin{array}{l}2.19 \\
2.14 \\
\\
2.12 \\
2.18 \\
2.12 \\
2.20 \\
2.20 \\
\\
2.29 \\
2.35 \\
2.48 \\
2.64 \\
2.84 \\
3.00 \\
3.15 \\
3.20 \\
3.17 \\
3.29 \\
4.94 \\
6.96 \\
9.45\end{array}$ & $\begin{array}{r}57 \\
66 \\
90 \\
126 \\
180 \\
232 \\
286 \\
304 \\
293 \\
339 \\
1,370 \\
3,180 \\
5,820\end{array}$ & 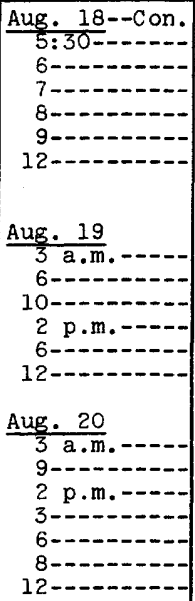 & $\begin{array}{l} \\
6.42 \\
6.01 \\
5.60 \\
5.25 \\
4.94 \\
4.55 \\
\\
4.40 \\
4.16 \\
3.95 \\
3.80 \\
3.65 \\
3.75 \\
3.68\end{array}$ & $\begin{array}{l}7,790 \\
6,660 \\
5,510 \\
4,600 \\
3,960 \\
2,920\end{array}$ & 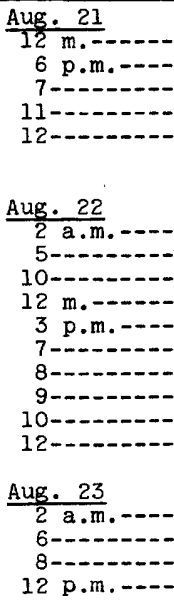 & $\begin{array}{l}3.52 \\
3.39 \\
3.28 \\
3.14 \\
3.08 \\
3.14 \\
3.39 \\
4.26 \\
4.33 \\
4.01\end{array}$ & $\begin{array}{l}354 \\
309 \\
273 \\
229 \\
202 \\
229 \\
309 \\
680 \\
718 \\
557\end{array}$ \\
\hline
\end{tabular}

(312) Lackawanna River at Old Forge, Pa.

Location.--Lat $41^{\circ} 21^{\prime} 30^{\prime \prime}$, long $75^{\circ} 44^{\prime} 40^{\prime \prime}$, on right bank $150 \mathrm{ft}$ upstream from Delaware, Lackawanna and Western Rallroad bridge in 0ld Forge, Lackawanna County, and 0.5 mile upstream from St. Johns Creek. Datum of gage is $595.26 \mathrm{ft}$ above mean sea level, datum of 1929, supplementary adjustment of ig43.

Drainage area. $--332 \mathrm{sq} \mathrm{mi}$

Gage-height record.-Water-stage recorder graph except for period 10 p.m. Aug. 18 to $2: 20$ p.m. Aug. 22 . Graph for period Aug. 19-22 drawn on basis of observer's reading, floodmark of peak and comparison with record for gaging station at Archbald.

Discharge record.--Stage-discharge relation defined by current-meter measurements below 5,000 cfs and extended to peak stage on basis of slope-area determinations at gage helghts 15.30 and $20.05 \mathrm{ft}$. Doubtful gage-helght record Aug. 1-11, 23-31.

Maxima.-August 1955: 31,000 cfs about 2 a.m. Aug. 19 (gage helght, 20.05 ft, from floodmark). 1938 to July 1955: Discharge, 20,900 cfs May 23, 1942 (gage height, 15.30 ft ), from slope-area determination of peak flow.

Mean discharge, in cubic feet per second, August 1955

\begin{tabular}{|c|c|c|c|c|c|c|c|c|c|c|c|}
\hline Day & $\mathrm{Cfs}$ & Day & $\mathrm{Cfs}$ & Day & Cfs & Day & Cfs & Day & Cfs & Day & $\mathrm{Cfs}$ \\
\hline $\begin{array}{l}1 \\
2 \\
3 \\
4 \\
5 \\
6\end{array}$ & $\begin{array}{l}102 \\
117 \\
117 \\
122 \\
125 \\
125\end{array}$ & $\begin{array}{r}7 \\
8 \\
9 \\
10 \\
11\end{array}$ & $\begin{array}{l}153 \\
122 \\
110 \\
110 \\
190\end{array}$ & $\begin{array}{l}12 \\
13 \\
14 \\
15 \\
16\end{array}$ & $\begin{array}{r}188 \\
1,060 \\
2,630 \\
1,870 \\
922\end{array}$ & $\begin{array}{l}17 \\
18 \\
19 \\
20 \\
21\end{array}$ & $\begin{array}{r}613 \\
4,820 \\
14,000 \\
3,680 \\
2,300\end{array}$ & $\begin{array}{l}22 \\
23 \\
24 \\
25 \\
26\end{array}$ & $\begin{array}{r}2,420 \\
2,090 \\
1,540 \\
1,170 \\
916\end{array}$ & $\begin{array}{l}27 \\
28 \\
29 \\
30 \\
31\end{array}$ & $\begin{array}{l}815 \\
695 \\
580 \\
534 \\
644\end{array}$ \\
\hline
\end{tabular}

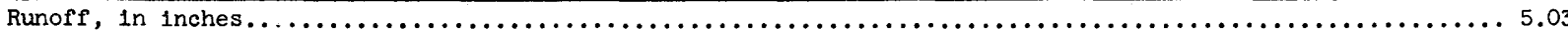

Gage height, in feet, and discharge, in cublc feet per second, at indicated time

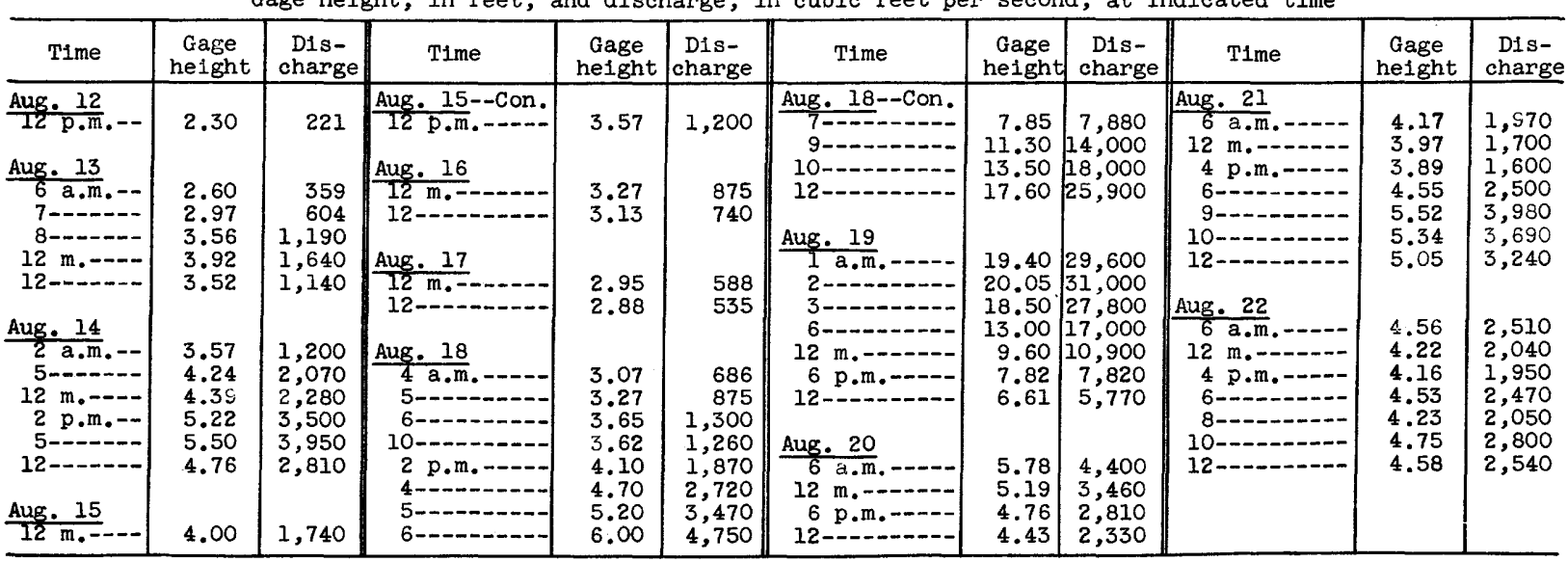


(316) Wapwallopen Creek near Wapwallopen, Pa.

Location.--Lat $41^{\circ} 04^{\prime} 35^{\prime \prime}$, long $76^{\circ} 05^{\prime} 40^{\prime \prime}$, on left bank $100 \mathrm{ft}$ upstream from Harts Bridge, $2 \frac{1}{4}$ miles southeast of Wapwailopen, Luzerne County, and 3.7 miles upstream from mouth. Datum of gage is $752.41 \mathrm{f} t$ above mean sea level (Pennsylvania Rallroad benchmark).

Drainage area. $--45.8 \mathrm{sq} \mathrm{mi}$.

Gage-helght record.--Water-stage recorder graph.

Discharge redord. - Stage-discharge relation defined by current-meter measurements below 1,300 cfs and extended to peak stage by logarithmic plotting. Shifting-control method used at times.

Maxima.--August 1955: D1 scharge, 3,140 cfs $11 \mathrm{p} . \mathrm{m}$. August 18 (gage helght, 9.23 ft).

1919 to July 1955: Discharge, 2,980 cf s July 22, 1952 (gage helght, $8.96 \mathrm{ft}$ ), as explained above. Mean discharge, in cublc feet per second, August 1955

\begin{tabular}{c|c||r|r|r|r||r|r||r|r|r||r}
\hline Day & Cfs & Day & Cfs & Day & Cfs & Day & Cfs & Day & Cfs & Day & Cfs \\
\hline 1 & 2.0 & 7 & 2.3 & 12 & 9.3 & 17 & 11 & 22 & 357 & 27 & 54 \\
2 & 1.7 & 8 & 4.5 & 13 & 74 & 18 & 810 & 23 & 183 & 28 & 46 \\
3 & 1.7 & 9 & 5.1 & 14 & 53 & 19 & 1,170 & 24 & 116 & 29 & 40 \\
4 & 1.5 & 10 & 3.4 & 15 & 22 & 20 & 310 & 25 & 84 & 30 & 46 \\
5 & 1.4 & 11 & 3.9 & 16 & 14 & 21 & 208 & 26 & 66 & 31 & 98 \\
6 & 1.7 & & & & & & & & & \\
\hline
\end{tabular}

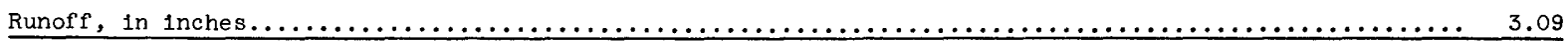

Gage helght, in feet, and discharge, in cubic feet per second, at indicated time

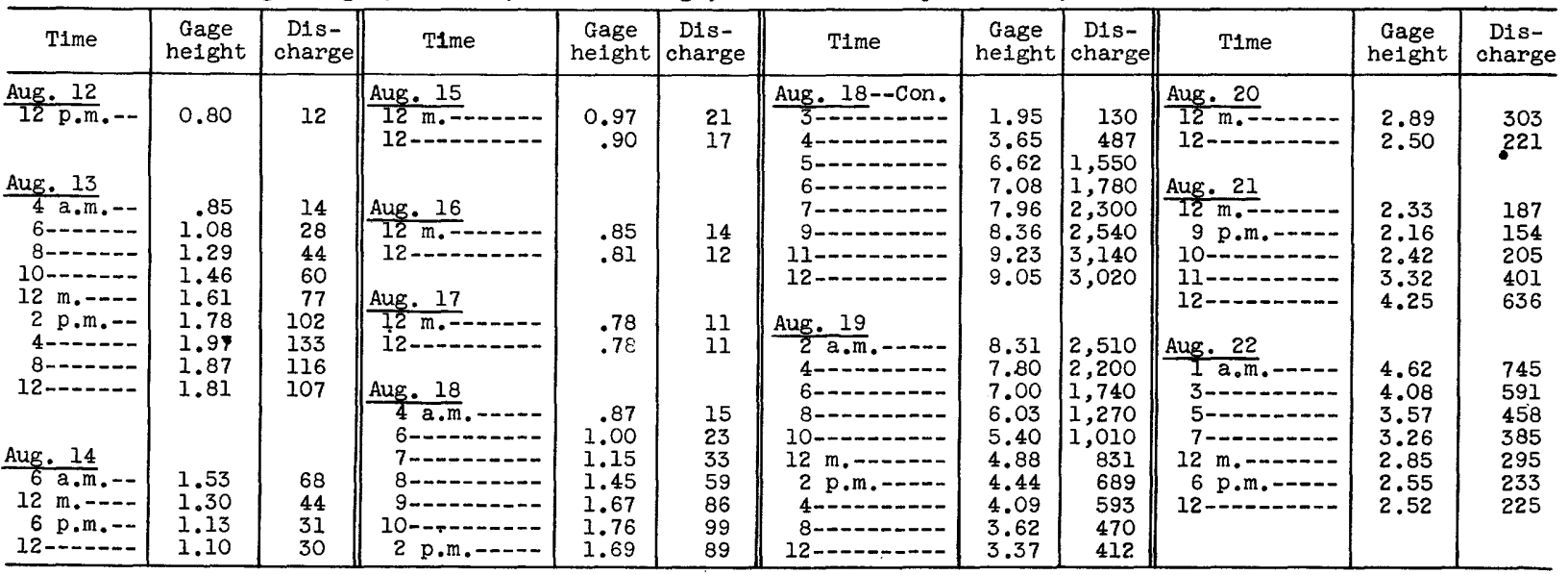

